Constantine the Philosopher University in Nitra

Faculty of Natural Sciences

\title{
Embryotechnology II
}

Jozef Laurinčík et al. 
Title: Embryotechnology II

Editor: Prof.h.c. prof. Dr. MVDr. Jozef Laurinčík, DrSc.

Reviewers: prof. MVDr. Peter Massanyi, DrSc.; Doc. Ing. Jozef Trandžík, PhD.

Publisher: Constantine the Philosopher University in Nitra, Faculty of Natural Sciences

Pages: 200

Print numbers: 50

Edition: Prírodovedec no. 681

(C) Faculty of Natural Sciences, Constantine the Philosopher University in Nitra 2018

DOI: http://doi.org/10.17846/2018-embryotechnology

ISBN 978-80-558-1337-0

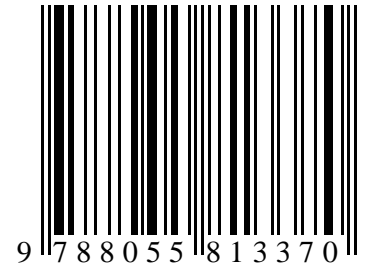

EAN 9788055813370

This work was supported by Slovak Research and Development Agency under the contract No. APVV-14-0001, and also by the projects "EXCELLENCE in molecular aspects of the early development of vertebrates", CZ.02.1.01/0.0/0.0/15_003/0000460 from the Operational Programme Research, Development and Education and by the Danish Council for Independent Research / Natural Sciences (FNU) 8021-00048B and was co-funded by the projects VEGA 1/0022/15, VEGA $1 / 0327 / 16$ and 1/0001/19. 


\section{Preface}

Technological and sciential progress in embryology during the past 30 years have been extraordinary, and it is predicted that the pace of new developments in this area will continue, or even accelerate. Despite of the enormous advance in the field of embryo technology, the oocyte and embryo itself, its survival of transfer, and the quality of the animal it produces still measure the success of any embryo manipulation. Therefore, progress in embryo technology can be evaluated in terms of how well the reproductive biologists can make and use embryos, and how much they have taught us about the maintenance of pregnancy and reproduction in general. Currently, the number of people employed in research with preimplantation embryos is approximately equal to the number employed in agricultural applications of embryo technology. Moreover, the number employed in applications of this technology in human medicine is increasing rapidly. Innovative advances in biotechnology and embryology will help us to understand many unsolved questions in reproductive biology, but also will raise new questions.

In order to elucidate and explain the basic principles of reproduction biology in mammals and describe the elementar and advanced embryo technologies including regulation of ovarian cycle, in vitro embryo production, cryopreservation of oocytes and embryos, micromanipulation techniques, transgenesis and epigenetics, the team of experts have set up the overview of each technology enhanced with inspiring discussions and general recommendation to use these technologies for the benefit of mankind when appropriate.

A special thanks go to all contributors for their time and effort spent during the production of this modern and unique compendium of information about embryo technologies. 


\section{PARTICIPANTS}

Jozef Laurinčík, Martin Morovič, Michal Benc - Department of Zoology and Anthropology, Faculty of Natural Sciences, Constantine the Philosopher University in Nitra, Slovak Republic František Strejček - Department of Botany and Genetics, Faculty of Natural Sciences, Constantine the Philosopher University in Nitra, Slovak Republic

Alexander V. Sirotkin, Peter Chrenek, Alexander V. Makarevich - Department of Genetics and Animal Reproduction, Research Institute of Animal Production, Slovak Agricultural Research Centre, Nitra, Slovak Republic

Jozef Laurinčík, Matej Murín - The Czech Academy of Sciences, Institute of Animal Physiology and Genetics , Rumburska 89, 27721 Libechov, Czechia

Martin R. Luck - Division of Animal Physiology, School of Biosciences, University of Nottingham, Loughborough, $U K$

Gábor Vajta - Cairns Fertility Centre, Corner McLeod and Aplin Streets, Cairns Queensland,4870, Australia

Lazo Pendovski - Ss. Cyril and Methodius University in Skopje, Republic of Macedonia Ol'ga Ostrup - Department of Basic Animal and Veterinary Sciences, Faculty of Life Sciences, University of Copenhagen, Groennegaardsvej 7, DK-1870 Frederiksberg C, Denmark 
Embryotechnology II 


\section{Contents}

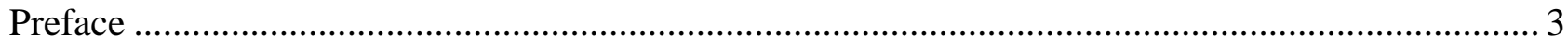

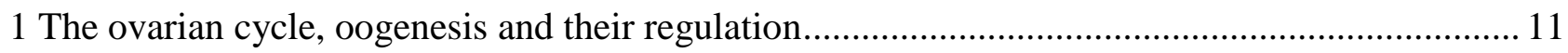

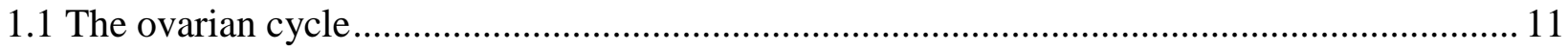

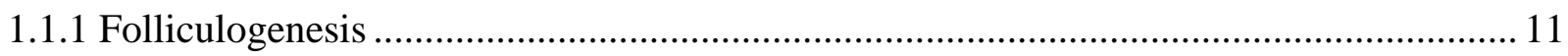

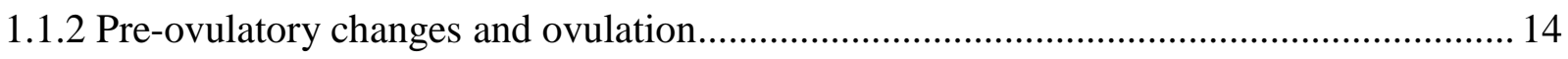

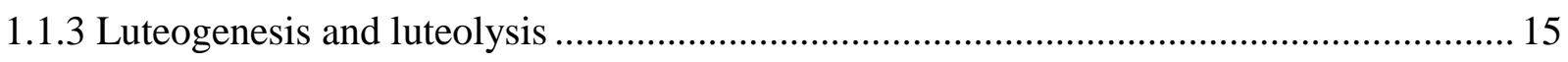

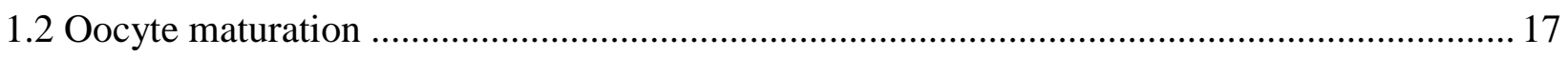

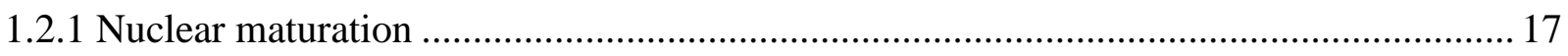

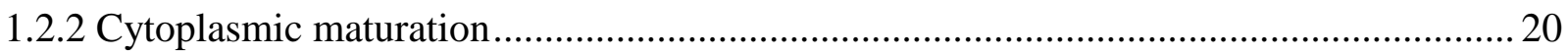

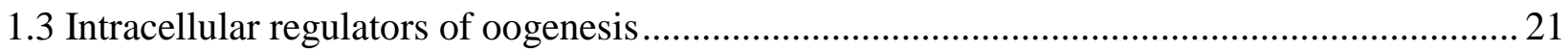

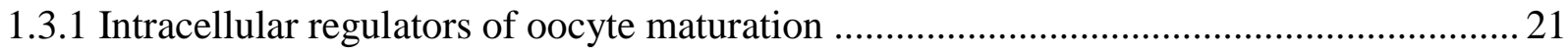

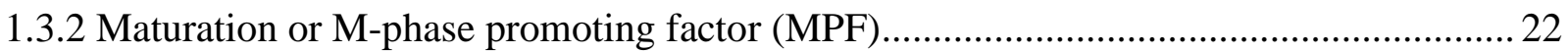

1.3.3 Mitogen-activated protein kinase (MAPK) …....................................................... 22

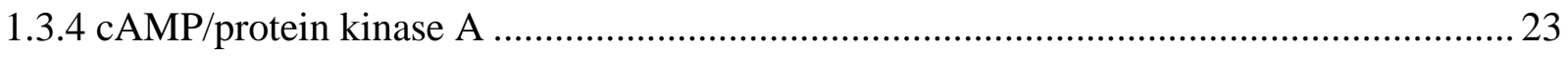

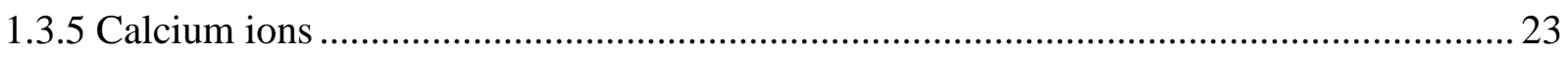

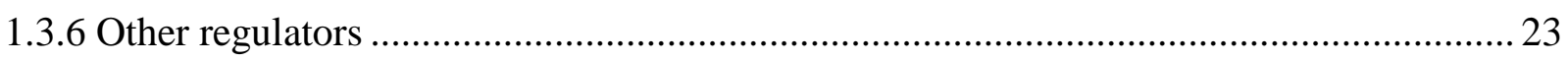

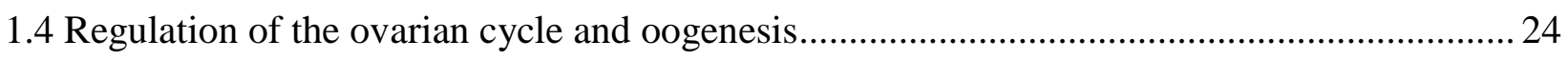

1.4.1 Regulation of primordial follicles and early oogenesis .......................................... 24

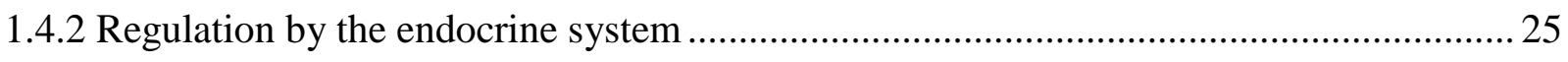

1.4.3 Regulation of primary and secondary/antral follicles .............................................. 26

1.4.4 Regulation of pre-ovulatory changes and ovulation ................................................ 28

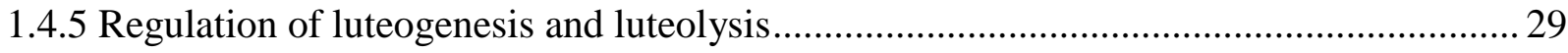

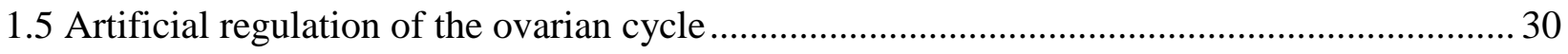

1.5.1 Synchronization of the ovarian cycle and contraception ........................................ 31

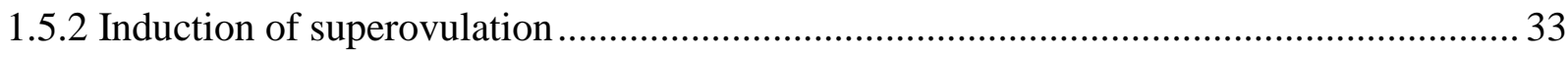

3 Quality of mammalian preimplantation embryos and its evaluation......................................... 53 


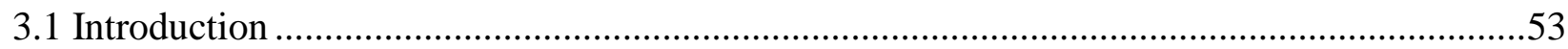

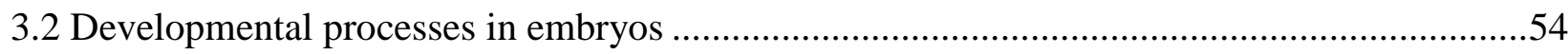

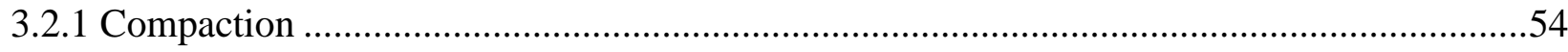

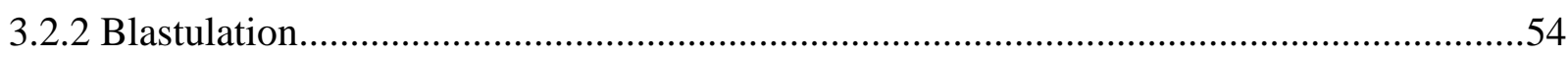

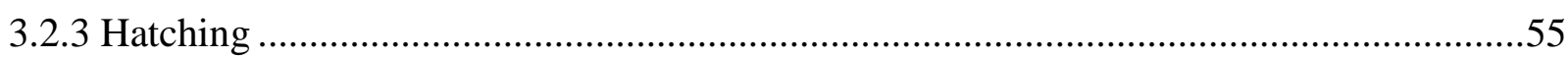

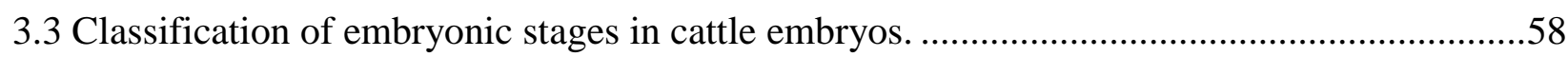

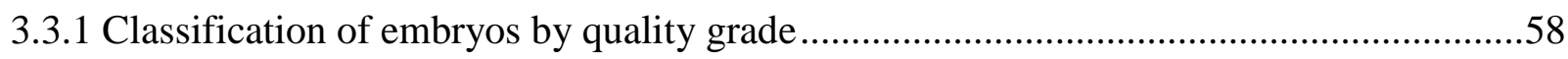

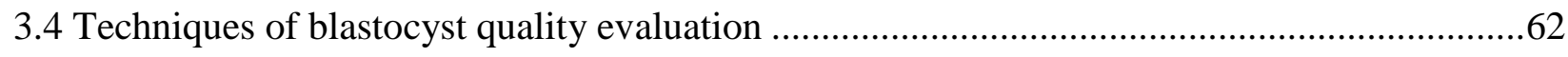

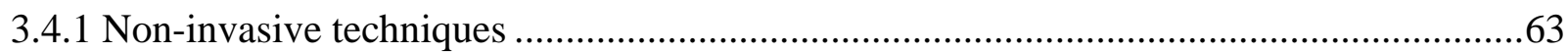

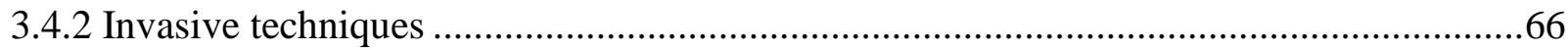

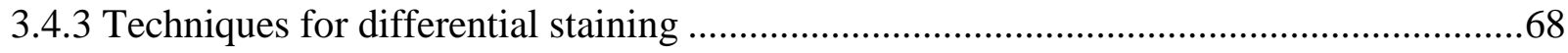

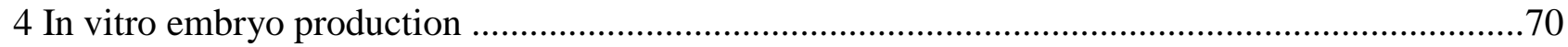

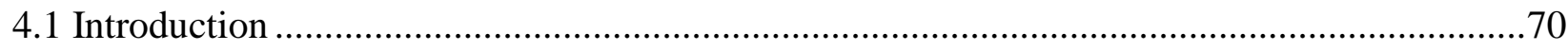

4.1.1 The history of in vitro embryo production. ........................................................... 70

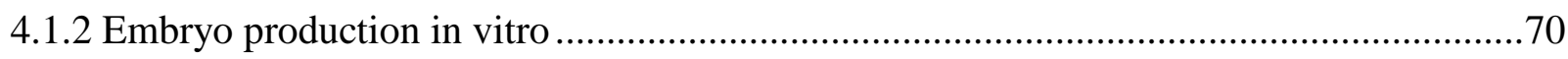

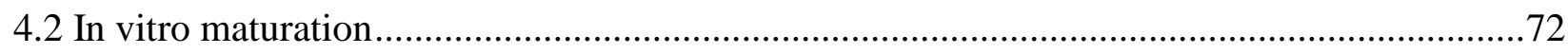

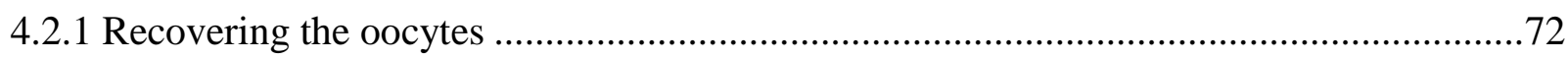

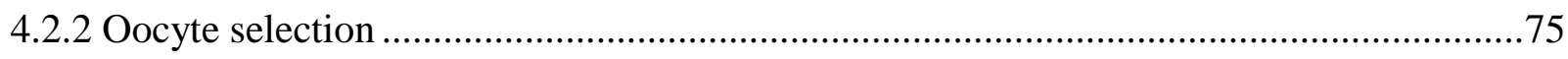

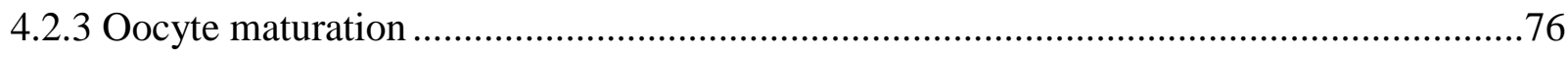

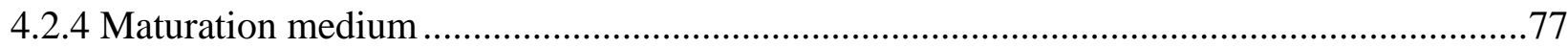

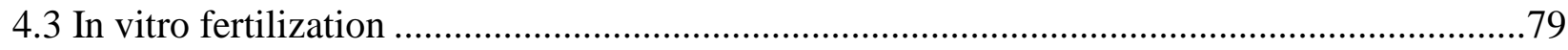

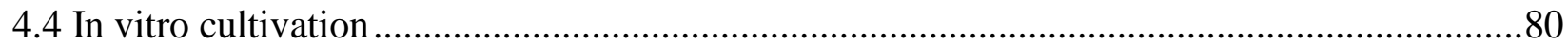

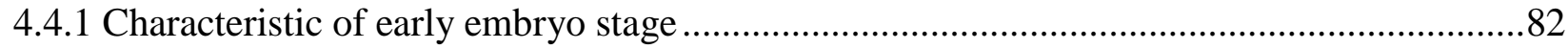

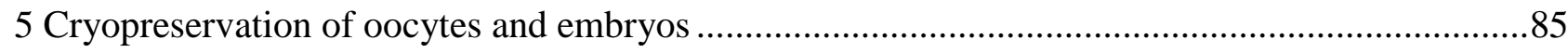

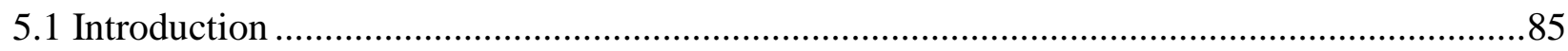

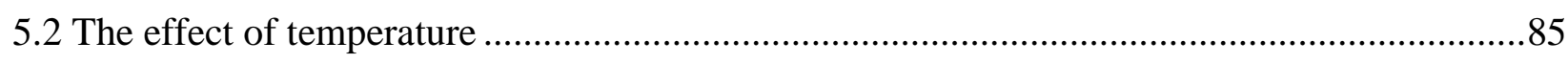

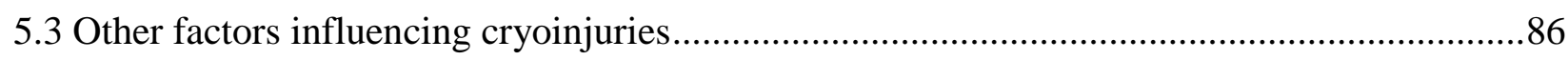




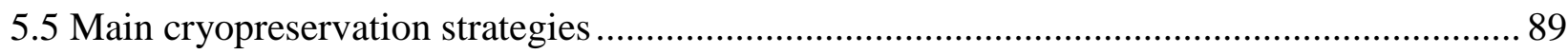

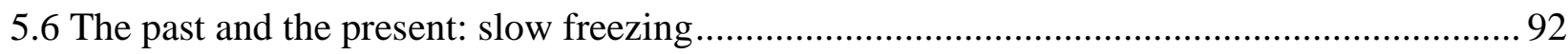

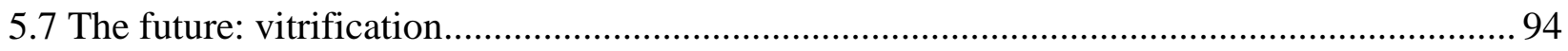

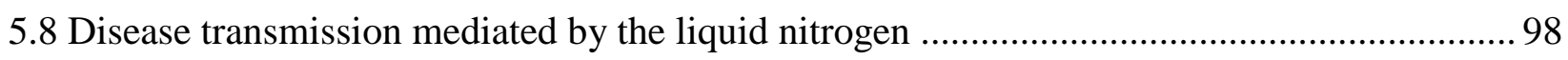

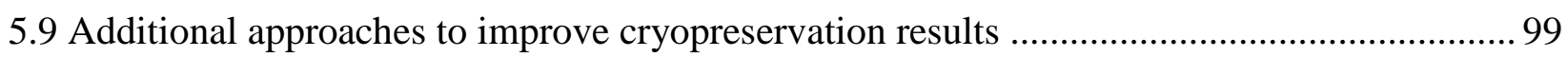

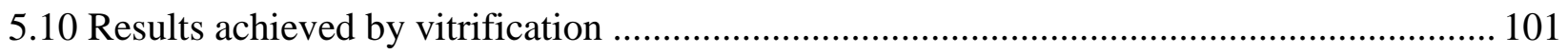

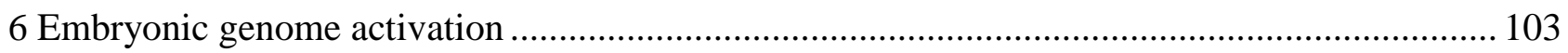

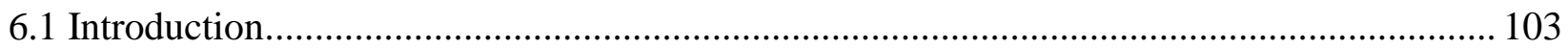

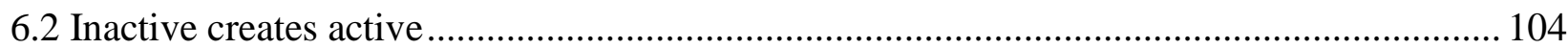

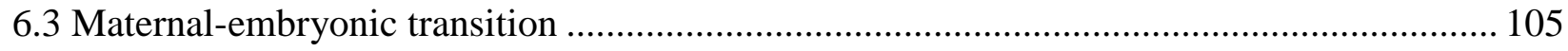

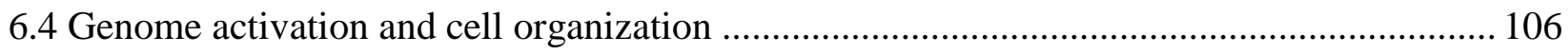

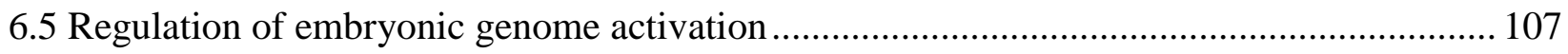

6.5.1 Maternal regulation of early development ........................................................... 107

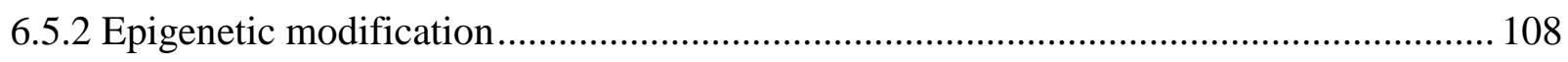

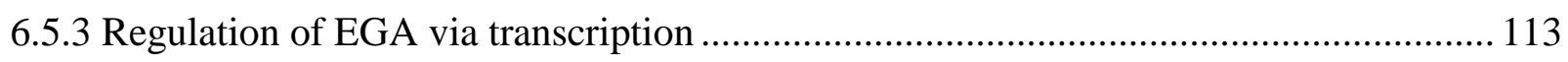

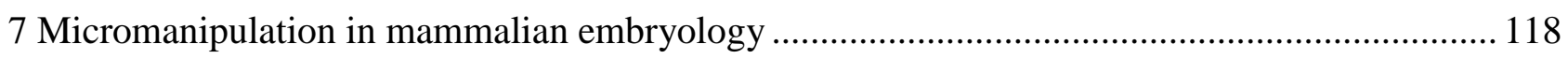

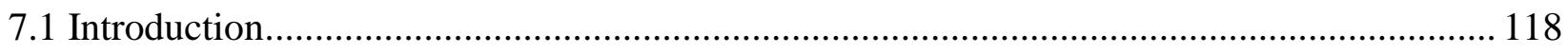

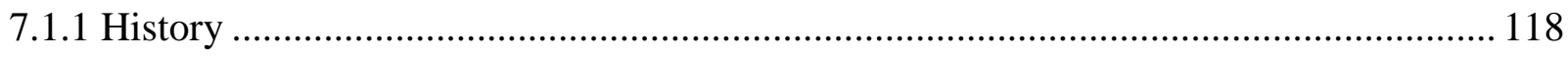

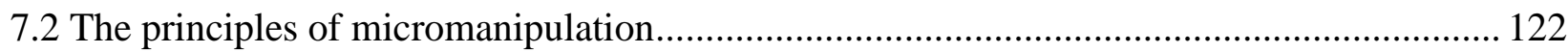

7.3 Application of micromanipulators in mammalian embryology ........................................ 126

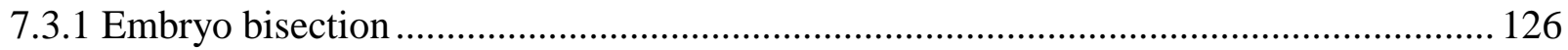

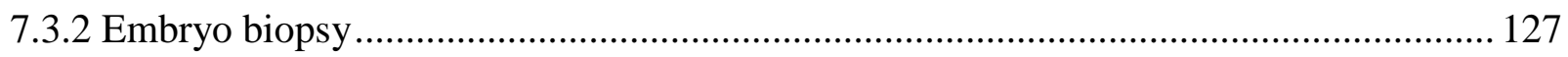

7.3.3 Subzonal and intracytoplasmic sperm injection ................................................. 128

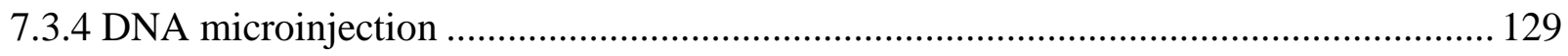

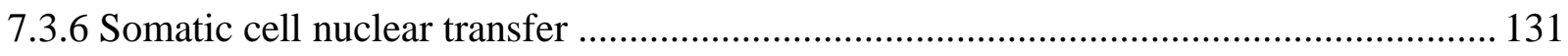

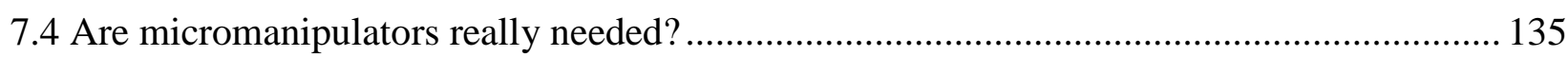




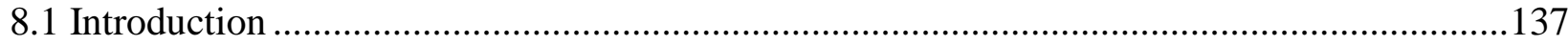

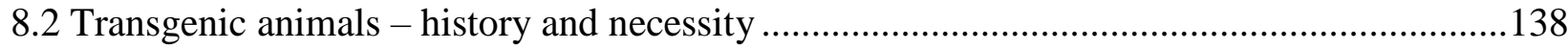

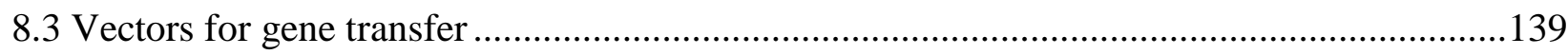

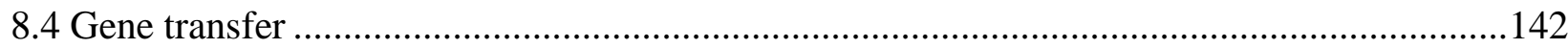

8.5 Factors influencing efficient production of transgenic animals .......................................148

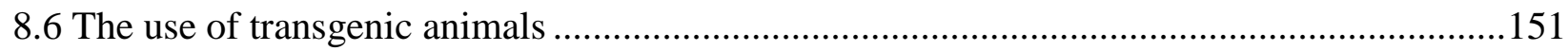

8.7 Methods for detection of transgene integration and expression........................................154

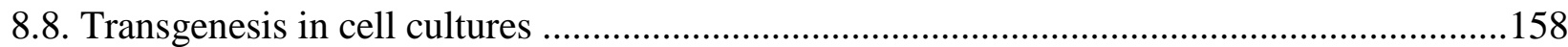

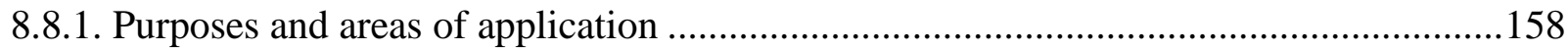

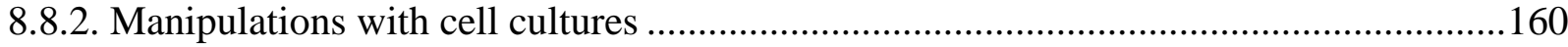

9 Nucleologenesis and nucleolotransfer in mammalian oocytes ..............................................162

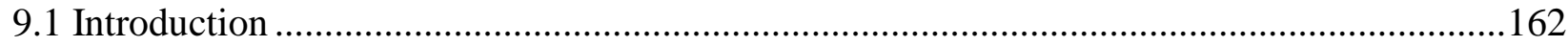

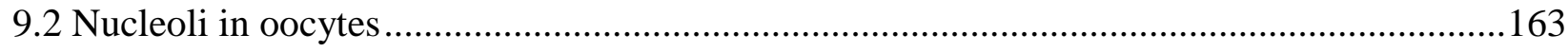

9.3 Nucleoli in zygotes and early cleavage-stage embryos.................................................165

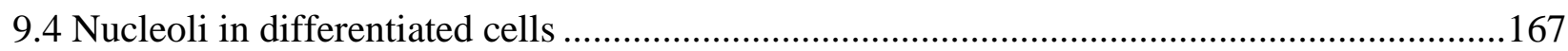

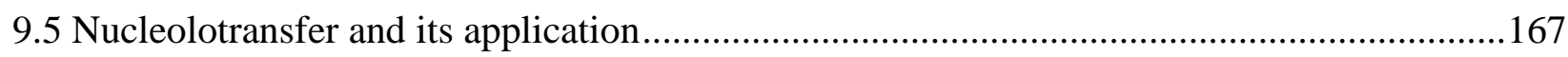

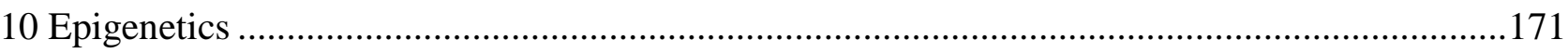

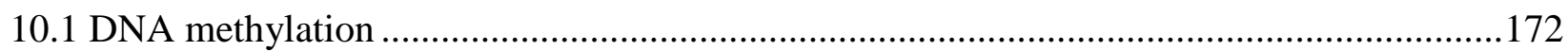

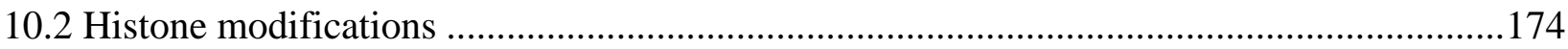

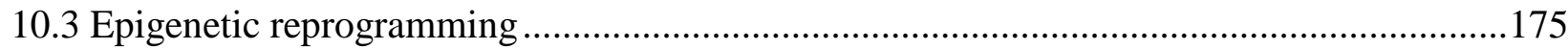

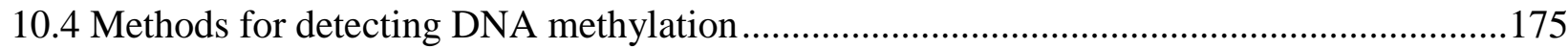

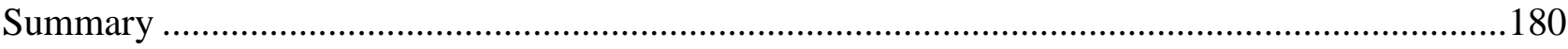

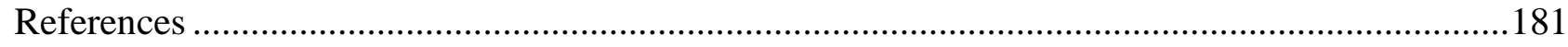





\section{The ovarian cycle, oogenesis and their regulation}

\subsection{The ovarian cycle}

The ovary is the central organ of the female reproductive system. It has two main functions: the production of gametes (oocytes) and the secretion of signaling and regulatory substances. The secretions of the ovary influence other parts of the reproductive system, including those which control female maturation, cyclicity, gamete production and behavior (brain, pituitary gland, the ovary itself), and those which support embryo development, gestation and lactation (reproductive tract, uterus, mammary gland). The ovary has two structural regions: the outer cortex contains follicles at various stages of development together with structures derived from follicles, whilst the inner medullar consists mainly of stromal tissues and vascular elements (Fig. 1.1).

\subsubsection{Folliculogenesis}

The principal functional unit of the ovary is the follicle. Primordial germ cells (precursors of oocytes) appear within the embryonic ovary and multiply by mitosis during prenatal ontogenesis. During postnatal ontogenesis, several thousands of germ cells cease their mitosis and begin meiosis (see below), becoming oogonia and then oocytes. Neighboring ovarian cells differentiate into follicular somatic cells, under the influence of oogonia/oocyte-derived factors.

Each oocyte becomes enclosed by one layer of flat follicular cells to form a primordial follicle. Subsequent proliferation of follicular cells leads to the formation of a primary follicle surrounded by several layers of cuboidal, epithelial cells. Under the influence of the oocyte, which is now in a state of meiotic arrest, the cells differentiate and produce a membrane made of protein and glycoprotein called the zona pellucida; they also supply the oocyte with nutrients and secrete signaling substances, including hormones such as estrogen, which regulate oogenesis and other components of the reproductive system.

The single layer of cells immediately adjacent to the oocyte, the corona radiata, have long processes which penetrate the zona pellucida and connect with the oocyte by gap junctions. The layers of more elongated cells surrounding these are called granulosa cells (and form the zona granulosa or stratum granulosum) and they initially fill the space between the corona cells and the outer basement membrane (basal lamina or membrana basalis) of the follicle. The outermost (mural) layer of granulosa cells are columnar rather than cuboidal and have intimate contact with the extracellular matrix components which make up the membrane (including collagen Type IV, laminin and heparan sulfate proteoglycan). 
The basement membrane is synthesized by the granulosa cells, working in cooperation with cells on its outer side which make up the theca interna. The theca interna is comprised of several different types of cell including fibroblasts, capillary endothelial cells and endocrine cells. The latter secrete a range of steroid and protein hormones, some of which may be transported across the basement membrane to regulate the activity of the granulosa cells and the oocyte and to be converted into other hormones. For example, in many species thecal androgen is converted by granulosa cells into estrogens which is then carried into the general circulation. A further external layer of tissue, the theca externa, consists of structural cells, connective tissue and capillaries. The latter supply the whole follicle with nutrients, gases and extra-ovarian signaling molecules. They reach into the theca interna but do not penetrate the follicular basement membrane. This means that the internal parts of the follicle (granulosa, corona, oocyte) are avascular and must receive blood-born nutrients, gases and other substances by transfer across the follicle wall.

During early follicular growth, granulosa cells divide and fill the expanding follicle. At a certain stage of growth, the volume of the follicle increases more rapidly than the total volume of the proliferating granulosa cells. Some inner layers of cells may also die or fail to replicate, possibly because of limited nutrient availability. At this stage, the differential volume of the expanding follicle fills with a fluid (follicular fluid or liquor folliculi) and a chamber or antrum is formed which largely separates the outer granulosa cell layers from the oocyte and corona. Some granulosa cells may remain contiguous with the corona cells, forming a short stalk called the cumulus oophorus. Follicles reaching this stage of development are called secondary or antral follicles.

The follicular fluid of an antral follicle is essentially a transudate of serum, derived from the thecal capillaries. It is partially filtered during its passage across the capillary endothelium, through the theca and across the follicle wall, and it is further modified by secretions from the granulosa cells and oocyte. It therefore contains small to medium sized serum factors, supplemented with relatively high concentrations of ovarian hormones and other follicle-derived products. The granulosa cells produce proteoglycans which swell up with water to form very large molecules; these are in particularly high abundance in follicular fluid. 


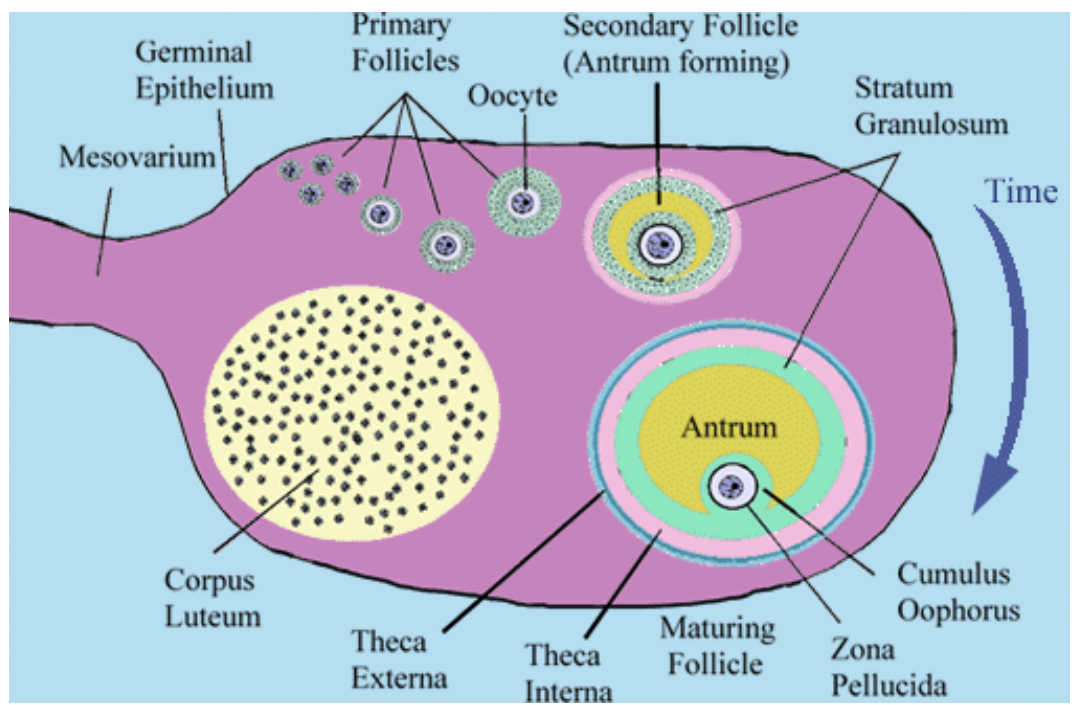

Figure 1.1: Ovarian structures and their development from primary follicle up to corpus luteum. (http://www.uoguelph.ca/502.html)

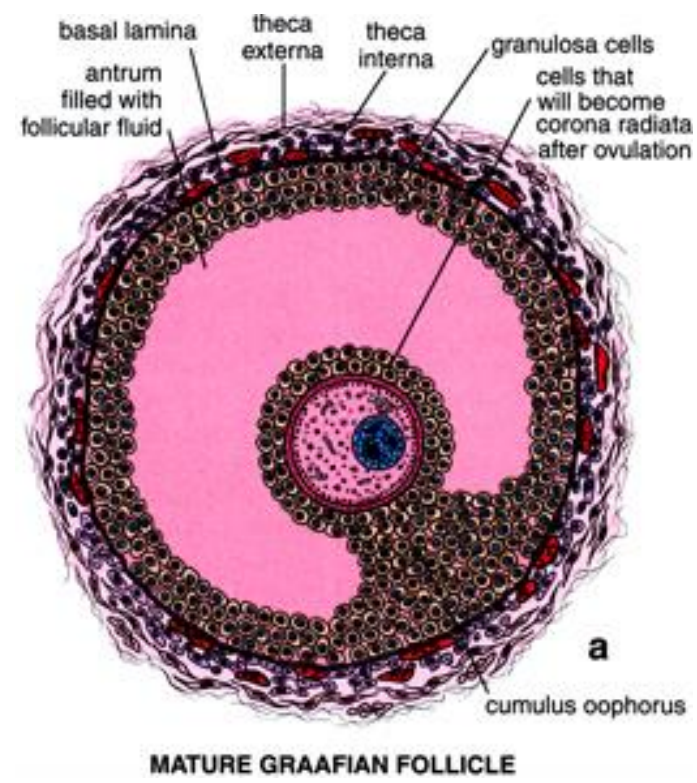

Figure 1.2: Structure of mature (Graafian) follicle http://upload.wikimedia.org/wikipedia/ en/5/50/Mature_Graffian_follicle.jpg)

At each stage of folliculogenesis, from the primordial and primary stages and through each level of antral growth, there is an intensive selection and loss of follicles. As a result, more than $99 \%$ of the primordial follicles originally present in the ovary are subjected to degeneration (known as atresia) due to programmed cell death (apoptosis). Those that survive selection have the potential to ovulate, 
depending on stage of the ovarian cycle at which they mature and the reproductive characteristics of the species. A large, fully differentiated antral follicle reaching the preovulatory stage of development is called a Graafian follicle (Fig. 1.2).

Perhaps a curious feature of ovarian science is that almost nothing is known about the tissue processes by which follicle growth occurs. This is despite our extensive knowledge of how this and other aspects of folliculogenesis are controlled by hormones and local factors (described below). It is unknown, for example, whether follicles grow as a cellular response to the pressure of fluid accumulating in the antrum, or whether fluid enters passively to fill the expanding volume. The former possibility seems the more likely, but it in turn raises the question of what causes the fluid to accumulate. We also need to understand what controls the movement of fluid from the blood as it passes across the capillary endothelium, through the theca and across the follicle wall. The basement membrane of the wall ultimately determines the integrity of the follicle prior to ovulation. This extremely fine structure, separating the theca and granulosa layers, grows rapidly as a sphere, without elastic stretching or change in thickness: there must be remarkable coordination between the granulosa and theca cells so that its collagen, laminin and proteoglycan constituents can be continuously deposited and remodeled without loss of integrity. These crucial growth processes are little understood.

\subsubsection{Pre-ovulatory changes and ovulation}

Ovulation is the process of tissue remodeling by which the cumulus-oocyte complex and follicular fluid escape the confines of the ovary and become available for fertilization. Some hours before ovulation, ovarian cells undergo dramatic morphological and functional changes, including the resumption of meiosis by the oocyte. The oocyte nucleus, or germinal vesicle, undergoes a series of events that involve the destruction of its membrane (called germinal vesicle breakdown, GVBD), and meiosis continues from diplotene-dictyotene to the second metaphase (first polar body) stage. Meiosis is then again arrested and will proceed no further unless the ovulated egg is fertilized. Meiosis is described in more detail below.

During these changes in the oocyte, the surrounding cumulus granulosa cells undergo mucification followed by expansion. The onset of mucification is marked by a dramatic increase in the secretion of the glycosaminoglycan hyaluronic acid into the extracellular spaces. Hyaluronic acid absorbs a large quantity of water and forms a bulky gel, causing the egg-cumulus complex to expand tremendously and leading to the dispersal of the cumulus cells. Cumulus expansion and the accumulation of hyaluronic acid are physiologically important as preparations for ovulation and 
subsequent fertilization: they allow the attachment and penetration of sperm, and the glycosaminoglycan induces the acrosome reaction in the sperm head prior to fertilization itself.

Immediately before ovulation, proteolytic and collagenolytic enzymes degrade the basement membrane between the theca and granulosa layers of the follicle wall. This allows capillaries to penetrate the antrum for the first time and the granulosa and thecal cells begin to mix. There is also a complex series of tissue events within the follicle wall and adjacent parts of the ovarian stroma which strongly resemble those of the inflammatory reaction. Enzymes in the wall of the ovary above the protruding follicle are activated in response to granulosa-derived hormones (including prostaglandin $\mathrm{F}$ and progesterone) and products from the invading blood cells; this causes a hole to form in the ovarian surface, called the stigma or macula pellucida. The follicular fluid is extruded through the stigma, under residual pressure from the collapsing follicle, taking with it the mature oocyte-cumulus complex which is then gathered by the fimbriae of the oviduct (Fallopian tube).

\subsubsection{Luteogenesis and luteolysis}

After ovulation, the follicle wall in the majority of mammalian species develops into a new tissue called the corpus luteum. This is a large, rapidly growing but transient endocrine gland that secretes progesterone, oestradiol and several protein hormones during the first part of the luteal phase of the cycle. These hormones prepare the uterine lining for implantation, and if the oocyte is fertilized and pregnancy ensues, the corpus luteum will continue to produce large amounts of progesterone, to maintain the condition of the uterus and support pregnancy. At the start of the luteinization of the ruptured follicle, a fibrin clot forms in the collapsed antrum (where the follicular fluid was previously located). This becomes a focus for the invasion of blood cells and the formation of a loose connective tissue in a process resembling that of post-inflammatory wound repair. Subsequently, a more solid tissue is established in which the mixing granulosa, theca and non-endocrine stromal cells are reorganized around an expanding network of capillaries. This is now the active corpus luteum.

During luteinization, the granulosa cells undergo cytoplasmic expansion and increase in size, now being referred to as large luteal or granulosa-lutein cells. They have a high capacity for progesterone synthesis. The theca (or theca-interstitial) cells are also incorporated into the corpus luteum but are smaller and described as small luteal or theca-lutein cells. They secrete lower but still significant amounts of progesterone and may also produce androgens. The extent to which the large and small luteal cells intermingle and the microanatomy of the luteal tissue varies between species.

As well as conditioning the uterus to support implantation and pregnancy, progestagens and other hormones from the corpus luteum limit the development of other follicles, thereby preventing maturation and ovulation until the corpus luteum regresses (luteolysis). In the event of pregnancy, the 
corpus luteum survives for the whole of gestation or until the placenta takes over the bulk of hormone secretion, depending on the species. If the oocyte is not fertilized and implantation does not occur, the corpus luteum degenerates within a fixed interval of time characteristic of the species. Histologically, the first indication of this is the apoptotic shrinkage of the large luteal cells. By contrast the small cells appear selectively hyperstimulated during early luteolysis. At the later stages of luteolysis both cell types become apoptotic and are eventually destroyed, leading to the morphological regression of the corpus luteum. All that remains is a nodule of dense, functionless connective tissue called the corpus albicans.

For primates, ruminants, rodents and the majority of other mammals, the ovarian cycle may therefore be described as having two basic phases: the follicular and luteal phases. The phases may be temporally distinct (for example, in humans), or there may be limited follicular development throughout the cycle with only the later stages of maturation being inhibited by the corpus luteum (for example, ruminants). An alternative description of the cycle, based on the behavior of the female and the cytological condition of the reproductive tract, but reflecting events in the ovary, includes the following phases: proestrus (start of follicular phase, luteolysis, ovaries secrete estrogen and small amounts of progesterone), estrus (completion follicular maturation, major secretion of estrogen, ovulation, mating, egg fertilization), metestrus (early luteal phase, time between ovulation and formation of corpus luteum, switch from oestrogen to progesterone secretion) and diestrus (active corpus luteum, highest rate of progesterone secretion). Anoestrus refers to times in an animal's life when estrous cycles are absent, for example before puberty, during pregnancy, at non-breeding times of year, or as a result of age or disease.

In humans, there is no estrous cycle as such because changes in sexual receptivity and genital appearance are absent or less evident than they are in most other species. There is a continuous sequence of alternating luteal and follicular phases of roughly equal length, separated by menstruation (designated as starting on Day 1 of the cycle) and ovulation (at "mid-cycle"). In other mammals, the start of the follicular phase (the start of proestrus) is designated by convention as Day 1 of the cycle. Some mammals (for example, rabbits) ovulate only in response to mating. Others (for example, rodents) form a corpus luteum only if the embryo implants and pregnancy takes place: no true corpus luteum appears after ovulation during an infertile ovarian cycle. Yet others (cats) may have an extended luteal phase ("pseudopregnancy") following an infertile mating. Many animals, including birds and lower vertebrates, do not form a corpus luteum at all.

Oocytes (eggs) are cells specialized for sexual reproduction, the main result of which is the pooling of genetic information from two organisms. As mentioned above, oocytes arise by mitosis from embryonic germ cells located in the embryonic ovarian cortex. Shortly before or after birth, 
mitotic activity ceases. The germ cells enter the prophase of meiosis and become oogonia, the precursors of oocytes. During subsequent growth and maturation, oogonia develop into mature oocytes. As with sperm, the maturation of oocytes includes both nuclear and cytoplasmic maturation.

To achieve the status of germ cell, a diploid somatic cell is transformed into a haploid cell. Fusion with another germinal haploid cell (that is, fertilization of oocyte by sperm), results in the formation a diploid cell (zygote) containing a mixed genome derived from both parent cells. The transformation of somatic cells into germinal cells includes a reduction in the number of chromosomes during the course of meiotic division (nuclear maturation; Fig. 1.3). There are also changes in cytoplasm which result in the accumulation of substances necessary for fertilization and for the formation of the zygote and first daughter somatic cells (cytoplasmic maturation). In contrast to male gametes (sperm), female gametes (oocytes) contain much more cytoplasm, organelles and stored material; this is due to unequal division (see below) and intensive protein synthesis at particular stages of the meiotic cycle.

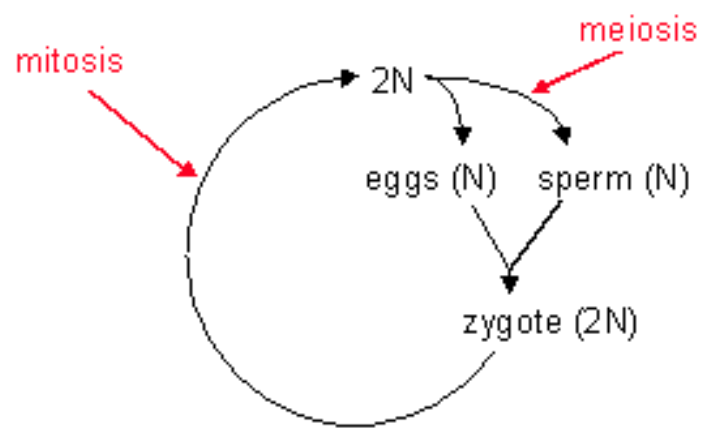

Figure 1.3: Transformation of diploid somatic cells to haploid gametes (eggs and sperm) during meiosis and return to diploid state after fertilization and zygote formation (http://faculty.clintoncc.suny.edu/faculty/Michael.Gregory/files/Bio\%20101/Bio\%20101\%20Lectur es/Meiosis/meiosis.htm)

\subsection{Oocyte maturation}

\subsubsection{Nuclear maturation}

The nuclear maturation of an oocyte occurs during meiosis. The phases of the cell cycle in meiosis are similar to those of mitosis (the division of somatic cells; Fig. 1.4):

synthetic (S) phase: the phase of duplication of DNA, synthesis of molecules required for division of mother cells, and distribution of genetic material between two daughter cells,

$>$ mitotic or meiotic $(\mathrm{M})$ phase: the phase of division of chromosome and cytoplasm, and their distribution between daughter cells, and 
growth or gap $(\mathrm{G})$ phases between these processes (G1 phase before, and G2 phase after, the S phase).

In contrast to mitosis, the $\mathrm{S}$ phase of meiosis is followed not by one, but by two subsequent divisions without an intermediary $\mathrm{S}$ phase, resulting in the formation of haploid rather than diploid cells.

The meiotic cell cycle is preceded by a premeiotic interphase in which the nuclear material is duplicated so that the cell, like all somatic cells, is diploid. There are then two cycles of meiotic division, meiosis I and meiosis II, separated by an interval called interkinesis. The meiotic phases have the same names as those of mitosis, plus an indicator of the division number:

meiosis I: prophase I, metaphase I, anaphase I, and telophase I meiosis II: prophase II, metaphase II, anaphase II, and telophase II

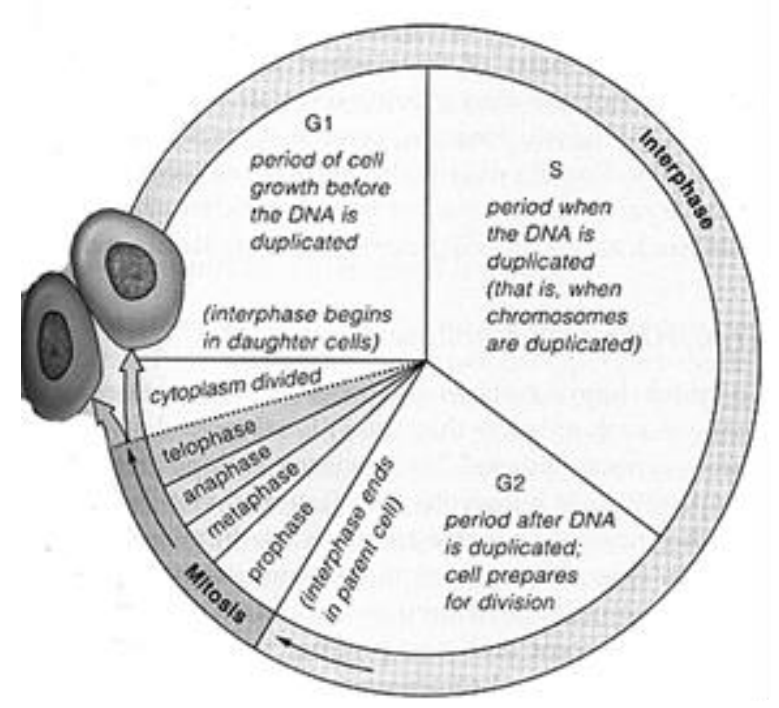

Figure 1.4: Phases of the mitotic cell cycle (http://ghs.gresham.k12.or.us/sciencel ps/sci/soph/cells/cycle/cyclerev.htm)

The main stages of meiosis are characterized by the following morphological features:

Diplotene - prophase of the first meiotic division; chromatin is diffuse, chromosomes are despiralised and the nuclear membrane (germinal vesicle, $\mathrm{GV}$ ) is present.

Diakinesis - the end stage of prophase of the first meiotic division; chromosomes (four copies of each - tetrads) are condensed and germinal vesicle breakdown (GVBD) occurs.

$>$ Metaphase I - maximal condensation of chromosomes and their localization to the equatorial area of oocyte; the end of GVBD; gap junctions between the oocyte and its cumulus oophorus cells break down. 
$>$ Anaphase I - the formation of a meiotic spindle and the separation of condensed chromosomes towards the poles of the oocyte.

$>$ Telophase I - chromosomes are localized at the poles of the oocyte; one of group of chromosomes starts to degenerate and form polar body I which is expelled to the perivitelline area between oocyte and zona pellucida.

Metaphase II - start of the second meiotic division of the surviving chromosome group. Chromosomes (two copies of each - diads) are localized in the equatorial area of the oocyte and a meiotic spindle is present.

$>$ Anaphase II - telophase II ; this is initiated by spermatozoa entering the cytoplasm of oocyte; it resembles anaphase I-telophase I.

In the first meiotic division, homologous chromosomes pair up and exchange segments, a process called recombination. The number of cells is then doubled but the number of chromosomes is not, resulting in half the number of chromosomes per cell. The second meiotic division is like mitosis in that the number of chromosomes does not get reduced.

The meiotic production of male germ cells (spermatozoa) results in the formation of four haploid cells for each initial diploid cell. In contrast, the production of female germ cells results in just one oocyte from each diploid cell. At each meiotic division, one of the pair of daughter cells develops whist the other, the "polar body", degenerates (Fig. 1.5). The meiotic distribution of cytoplasm between the daughter cells is unequal: the maturing cell receives the majority with a small amount going to form the polar body. The polar body with its degenerating chromosomes is expelled to the surface where it remains functionless and eventually disappears. During and after these events, chromosomes (each is present in one copy - monad) are despiralised within the haploid pronucleus. At fertilization, this fuses with a male haploid pronucleus to form the diploid nucleus of a zygote and a new diploid organism. 


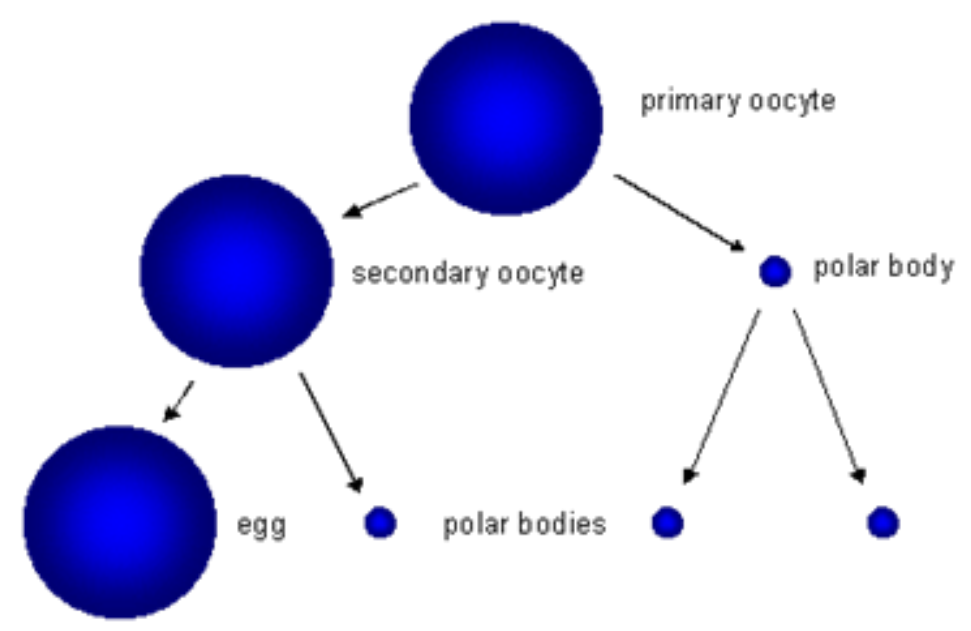

Figure 1.5: Division of cytoplasm during oocyte maturation: first and second meiotic divisions (http://faculty.clintoncc.suny.edu/faculty/Michael.Gregory/files/Bio\%20101/Bio\%20101\%20 Lectures/Meiosis/meiosis.htm)

In contrast to sperm, meiosis in oocytes is a prolonged process: after their formation within the embryo, oocytes remain at the G2 (diplotene-dictyotene) stage of meiosis I (with despiralised chromosomes surrounded by a germinal vesicle) until the animal reaches reproductive age. As the ovaries become active, oocytes leave this prolonged resting state and resume their growth and cytoplasmic maturation. Oocytes resume meiosis sequentially, in association with cycles of follicular development, and reach the second meiotic metaphase (first polar body stage) in the preovulatory follicle. Thus, between the onset of puberty and completion of folliculogenesis, each maturing oocyte undergoes GVBD, chromatin condensation (formation chromosomes) and two chromosome divisions; in other words, it progresses from diplotene-dictyotene through diakinesis, metaphase I, anaphase II and telophase II to reach metaphase II. At this point, meiosis is once again arrested and will only proceed (up to telophase II) if the ovulated egg is fertilized.

\subsubsection{Cytoplasmic maturation}

Cytoplasmic maturation refers to oocyte growth and the accumulation of biologically active substances necessary for further development. The growth of mammalian oocytes occurs in two distinct and characteristic stages. In the first, the growth is temporally correlated with that of the follicle in which it develops. In the second phase, the size of the oocyte remains constant, despite the continuing growth of follicle up to its pre-ovulatory condition. Macromolecules stored in oocytes during the first growth phase are responsible for progression through meiosis and will be required subsequently for the decondensation of penetrating spermatozoa, the formation of a male pronucleus, fertilization, the formation of the zygote and embryo up to the 6-8 blastomere stage. 
The successful growth and maturation of the oocyte within the follicle depend on the surrounding follicular somatic cells of the cumulus and granulosa layers. Somatic cell-oocyte interactions via gap junctions provide oocytes with energy and substrates for metabolism and for future divisions, fertilization and the early stages of embryogenesis. The materials supplied include nucleosides, amino acids, phospholipids and ions, along with paracrine/endocrine signaling substances which control oocyte maturation (meiosis regulating factors including cAMP, MPF etc: see below).

Any deficiency in the somatic cells or defects in the gap junctions between these cells and the oocyte will prevent optimal oocyte growth and maturation; this may result in an inability of the oocyte to undergo fertilization or may reduce the competence of the zygote and early embryo to embark successfully on further development. Thus the health of the follicle has a significant influence on the oocyte's potential to give rise to an offspring. The direct involvement of the follicle in oocyte development represents a route by which the health and physiological status of the female animal may determine the eventual quality of her offspring and the overall efficiency of reproduction. For scientists studying reproduction in the laboratory and embryologists carrying out assisted reproduction procedures, it is essential to obtain the best quality oocytes and to optimize the conditions under which they are handled and manipulated.

It should also be noted that the interrelationships between oocyte and follicular cells are bidirectional. The oocyte secretes specific signaling molecules (mainly growth factors and hormones) which can influence a range of ovarian processes including folliculogenesis and expansion, the secretion of steroid hormones by the cumulus oophorus, and the proliferation, differentiation, secretory activity and luteinization of granulosa cells.

\subsection{Intracellular regulators of oogenesis}

\subsubsection{Intracellular regulators of oocyte maturation}

Meiosis in oocytes has not only similar phases, but also similar control mechanisms to mitosis in somatic cells. In both types of division, progression from the G1 (or G0) phase of differentiated function or cytoplasmic growth, into the S phase of DNA replication prior to division (cytokinesis), is stimulated by the phosphorylation of proteins responsible for regulating the cell cycle. Protein phosphorylation is catalyzed by enzymes called protein kinases. The key protein kinase systems of cell division are MPF and MAPK. 


\subsubsection{Maturation or M-phase promoting factor (MPF)}

MPF is a protein kinase consisting of two subunits: a catalytic subunit called cyclin-dependent kinase (CDC kinase, $\mathrm{CDK}$ ) and a regulatory subunit called cyclin B. MPF is activated by the association of the subunits, and in both mitosis and meiosis the amount of cyclin present in the cytoplasm varies at different phases of the cell cycle. Thus cyclin can be thought of as turning CDK "on" or "off". There are two forms of MPF: one (cyclin E and CDK2) regulates G1 to S (from differentiation to DNA synthesis) and the other (cyclin B and CDK1) regulates the transition from G2 to M (the start of cell division). Each activated CDK then activates further cell cycle control proteins. The cell also has several inhibitory proteins which suppress the next stage if the previous stage is incomplete.

In oocytes, the CDK1 form of MPF can be activated by c-mos (cytostatic factor, CSF), an oocytespecific protein kinase which prevents degradation of cyclin B1. At the end of prophase I and diplotene-diakinesis-metaphase-anaphase I, MPF activity increases. This promotes GVBD and reinitiates the subsequent steps in meiosis, including chromosome condensation and spindle formation, up to metaphase I. It then inhibits DNA replication (S phase) between the first and second meiotic division. Transition from metaphase I to telophase I requires the inactivation of MPF, which is due to the proteolysis of cyclin B. A further activation of MPF occurs during metaphase II. This time, the elevated MPF holds the oocyte in metaphase II arrest until it is fertilized.

The amounts of active c-mos and MPF define both the meiotic competence and the cytoplasmic maturation potential of the oocyte. Oocytes with low meiotic and developmental competence may be deficient in MPF. After fertilization and the start of mitosis in the zygote, both c-mos and MPF are degraded; this allows the resumption of the $\mathrm{M}$ phase of the cell cycle and the normal mitotic divisions which lead to the formation of the early embryo.

\subsubsection{Mitogen-activated protein kinase (MAPK)}

The activation of MPF is promoted not only by c-mos, but by other protein kinases including mitogen activated protein kinases (MAPK). Increased accumulation of MAPK occurs during oocyte maturation in parallel to that of MPF, although in contrast to MPF there is a gradual increase in MAPK up to metaphase II. As with MPF, the activity of MAPK correlates with the oocyte's meiotic competence and degree of cytoplasmic maturation. MAPK phosphorylates and thereby activates cytoskeletal proteins and nuclear lamin, both of which play an important role in meiosis. The role of MAPK in cytoplasmic maturation is not fully understood. It may be a promoter of MPF formation, by phosphorylating/activating CDC2 kinase and by preventing the degradation of cyclin $\mathrm{B}$, although in some species the peak in MPF accumulation precedes that of MAPK. Moreover, MAPK can cause 
the reinitiation of meiosis in the presence of blocked MPF, suggesting that their actions are independent.

\subsection{4 cAMP/protein kinase A}

Cyclic adenosine monophosphate (cAMP) is a known inhibitor of oocyte nuclear maturation. It activates cAMP-dependent protein kinase A (PKA), which phosphorylates/activates proteins involved in the control of GVBD and the reinitiation the meiosis. Cumulus oophorus cells produce relatively large amounts of cAMP and transport it to the oocyte through gap junctions. High concentrations of cAMP keep the oocyte at the G (prophase/diplotene) stage of meiosis. This action may be mediated by PKA which can inhibit oocyte maturation by phosphorylating the CDC2 component of MPF. During the preovulatory period, the mucification and expansion of the cumulus oophorus is associated with destruction of gap junctions which prevents transport of cAMP to the oocyte. The decrease in cAMP and phosphorylation of its substrate PKA, removes signals blocking oogenesis and reinitiates meiosis.

\subsubsection{Calcium ions}

Oscillations in $\mathrm{Ca} 2+$ occur within the oocyte, depending on its stage of maturation and developmental competence. These oscillations cause activation of proteolytic enzymes which degrade c-mos (CSF) and MPF. The reduction in MPF and reinitiation of mitosis after fertilization may be due to a large increase in $\mathrm{Ca} 2+$ oscillations induced by penetrating spermatozoa. Any absence or abnormality in $\mathrm{Ca} 2+$ oscillations in oocytes matured in vitro can result in abnormal nuclear and cytoplasmic maturation, whilst stimulation of $\mathrm{Ca} 2+$ accumulation may increase an oocyte's potential for embryo development.

\subsubsection{Other regulators}

Several other potent regulators of oocyte maturation have been described. Oocyte maturation (or meiosis) inhibitor (OMI) is a peptide produced by theca and/or granulosa cells which suppresses nuclear maturation. Substantial amounts of OMI accumulate in follicular fluid and it can also be detected in medium following the experimental culture of follicular somatic cells. Follicular fluid also contains several meiosis activating sterols (MAS) which promote the reinitiation of meiosis.

Glycosaminoglycans produced by ovarian granulosa cells under the control of gonadotropins are able to prolong nuclear, but not cytoplasmic maturation. In addition, they affect ovarian steroidogenesis which in turn affects oocyte maturation. In lower vertebrates, nuclear and cytoplasmic 
maturation are stimulated mainly by the steroid hormone progesterone produced by surrounding ovarian cells. A number of other extra- and intracellular substances are able to affect oocyte maturation, but their physiological role and mechanisms of action require further research.

\subsection{Regulation of the ovarian cycle and oogenesis}

\subsubsection{Regulation of primordial follicles and early oogenesis}

The first stages of ovarian folliculogenesis (formation of primordial follicles) and oogenesis (formation of oogonia and start of meiosis) occur in the embryo, before the formation of endocrine system is complete. These stages of folliculo- and oogenesis are probably independent of hormones (see below) but under the control of local growth factors and related signaling molecule. These reach the developing gonadal cells via interstitial fluid and intercellular spaces and therefore act in an autoor paracrine manner. They are involved in the control of cell proliferation, growth and differentiation.

Amongst the most influential regulators are the insulin-like growth factors (IGFs). These are produced in a range of tissues throughout the developing embryo. IGFs produced by the follicular cells themselves ensure self-stimulation of follicular cell growth, whilst IGF binding proteins (IGFBPs), which are also produced by these cells, bind to and reduce the amount of free, biologically active IGFs and exert a regulatory function.

The bone morphogenetic proteins (BMPs) are another specific family of developmentally-crucial growth factors. They are secreted by the oocyte, and their receptors are important promotors of proliferation and differentiation in the surrounding follicular somatic cells.

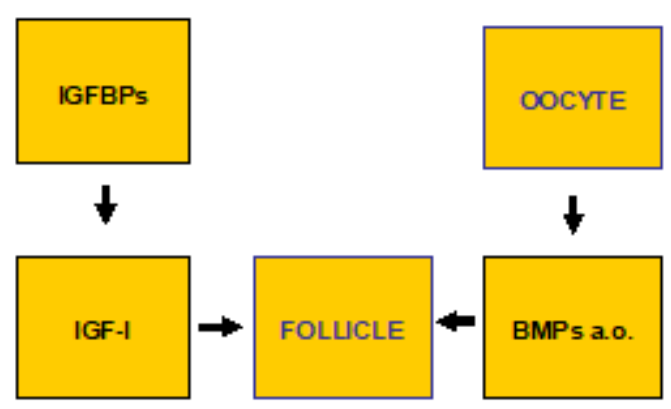

Figure 1.6: Regulators of the primordial follicle 


\subsubsection{Regulation by the endocrine system}

The development of primary and secondary follicles and the growth of the oocyte, as well as later reproductive events such as ovulation, luteal function and early pregnancy, are under the control not only of local hormones and growth factors but also of the organism's endocrine system. This develops embryologically at roughly the same time that the early gonad, with its full complement of germ cells and primordial follicles, is becoming established.

The endocrine glands produce hormones. These are messaging and signaling substances, transferred from one cell to another and carried by blood and other non-excreted fluids (lymph, cerebrospinal fluid, interstitial fluid etc.), sometimes over large distances through the body. The endocrine system includes peripheral hormone-producing cells (located in practically all tissues), a number of integrated organ and tissue systems, and the hypothalamo-hypophysial axis. The latter includes the pituitary gland (hypophysis) and the hypothalamus (a basal part of the brain) and provides one of the main links between the endocrine system and the central nervous system. Hypothalamic endocrine cells produce "releasing" and "inhibiting" hormones (sometimes also called factors) into a local region of the circulation which takes them directly to the cells of the anterior pituitary gland. Here they stimulate or inhibit the secretion of "tropic" hormones into the general circulation. The products of the anterior pituitary gland regulate a range of peripheral organs and are often described in the context of homeostasis, or the maintenance of the stability of the internal environment. The posterior part of the pituitary is actually a down growth of the hypothalamus itself and releases small peptide hormones (oxytocin, vasopressin) directly into the blood.

In the case of the reproductive system, the most important products of the anterior pituitary gland are the gonadotropins (follicle-stimulating hormone or FSH and luteinizing hormone or LH). Secretion of these glycoprotein molecules is stimulated by a small hypothalamic peptide hormone called GnRH (gonadotrophin releasing hormone). The production of $\mathrm{GnRH}$ is controlled by the brain (and ultimately by genetic, developmental and environmental signals) and by other hormones operating as components of feedback systems. The secretion of GnRH is pulsatile and its pulses cause the release of corresponding bursts of gonadotrophin.

In the male, gonadotrophins act on the testes to regulate sperm production and the secretion of male sex hormones (androgens) such as androstenedione and testosterone. In the female, they regulate the cyclical growth of follicles and the secretion of female sex hormones such as estradiol (an estrogen) and progesterone (a progestagen). They also initiate ovulation and help to maintain the production of hormones needed to establish and sustain pregnancy. In both sexes, gonadotropins regulate the onset of reproductive activity during puberty. Note that although the main sex hormones produced by the gonads (androgens, estrogens, progestagens) are often described by their role in the 
maintenance of gender phenotype, i.e. as "male or "female", they are secreted by both types of gonad, as are many other similar steroid hormones.

\subsubsection{Regulation of primary and secondary/antral follicles}

The hormonal control of follicle growth and development in the ovary is extremely complex. According to the "two cell-two gonadotropin" hypothesis, different follicular compartments are controlled by different gonadotropins. Thus, LH acts mainly on cells in the theca layer, resulting in the secretion of androgens, and FSH mainly stimulates granulosa cells, for example in the conversion of theca-derived androgens to estrogens. Whilst this hypothesis provides a framework for further understanding, it greatly oversimplifies the situation. The roles and sites of action of the gonadotrophins vary according to the stage of the cycle and the developmental status of the follicles. This is partly because the gonadotrophins themselves vary in concentration and partly because the cells of the ovary vary in their expression of the receptors needed in order to respond. The gonadotrophins also interact with a complex range of locally produced factors including the steroid hormones, a range of peptide hormones and a diverse array of growth factors. The following paragraphs describe some of that complexity, with particular reference to FSH and follicle survival.

FSH, secreted into the general circulation by the pituitary gland, reaches capillaries in the theca layers of ovarian follicles and then diffuses to the granulosa cells and follicular fluid. It can induce the proliferation, growth and differentiation of follicular cells and can interact with, and modify the actions of, locally secreted ovarian hormones and growth factors. These interactions define the maturational fate (towards ovulation or atresia) of follicles and therefore the general reproductive efficiency of the organism. Only a very small minority of follicles can survive to the large antral stage and become available for pre-ovulatory selection. Thus the number of offspring eventually produced reflects in part the balance between healthy and atretic follicles.

Healthy follicles are characterized by intensive cell proliferation, low rates of cellular apoptosis, an abundance of FSH receptors, intensive conversion of androgens to estrogens by granulosa cells, high cAMP production, and high levels of IGF-I. These characteristics are closely interrelated. Moderate concentrations of FSH promote estrogen, IGF-I and cAMP production, which in turn promote the formation of FSH receptors and thereby define the sensitivity of the follicle to FSH (a self-stimulating system). FSH-stimulated IGF-I also suppresses apoptosis and promotes cell proliferation, leading to follicle growth and development. The presence of active IGF-I in the follicle requires low levels of IGFBPs, which would otherwise bind and neutralize the growth factor. IGFBPs are themselves cleaved by specific proteases (pregnancy-associated plasma protein and other factors). 
Follicles stimulated by FSH produce a peptide hormone called inhibin. This feeds back to the pituitary and hypothalamus, prevents the overproduction of FSH and ensures a balanced level of ovarian activity. Granulosa cells within the resulting, sequentially developing follicles produce large amounts of cAMP which, amongst other actions, prevents the premature reinitiation of meiosis as described earlier. The cAMP accumulates in response not only to FSH, estrogens and IGF-I but also to many indirect regulators such as growth hormone, insulin and the appetite-related hormone leptin. These and similar interactions offer a route by which the health and metabolic status of the mother may influence reproduction. There is growing evidence that they may even determine the subsequent expression of specific genes in the early embryo, developing young and eventual adult.

As indicated previously, the estrogens secreted by growing follicles have numerous actions outside the ovary. They promote the development and function of other reproduction-related cells, tissues and organs including the oviduct and uterus, and they maintain secondary sexual features of phenotype including those in the brain responsible for sexual behavior. Estrogens are also essential for many physiological processes not directly related to reproduction, such as bone formation, the elasticity of skin tissue and aspects of mental health. Many of these extra-ovarian oestrogen effects are mediated through elevated cAMP production and involve interactions with other parts of the endocrine and nervous systems.

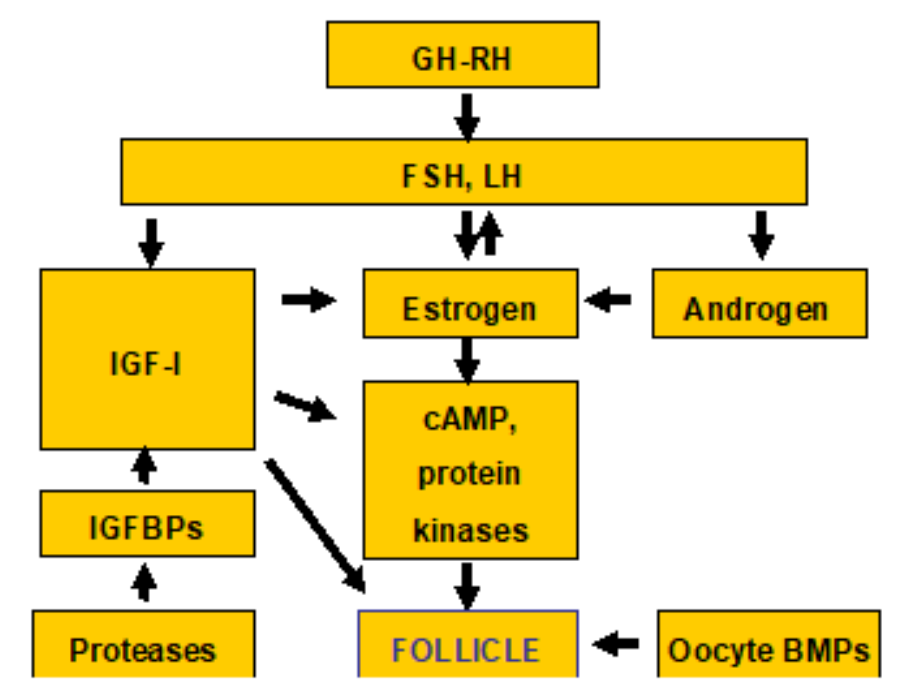

Figure 1.7: Regulators of primary and secondary/antral follicles 


\subsubsection{Regulation of pre-ovulatory changes and ovulation}

The transformation of a growing antral follicle into a preovulatory Graafian follicle is associated with rising secretions of steroid and peptide hormones. These hormones stimulate the release of both hypothalamic GnRH and pituitary FSH, and shortly before ovulation the size and frequency of secretory pulses of both hormones increase dramatically. The preovulatory surge in FSH secretion induces expansion of the cumulus oophorus surrounding the oocyte, and also increases the secretion of inhibin and oestrogen by follicular cells. Inhibin, as a suppressor of FSH output, terminates the preovulatory FSH surge. Estrogen, in the presence of the large amount of secreted GnRH, induces a preovulatory peak in LH secretion, which follows closely on the FSH peak. The estrogens and FSH together increase the production of cAMP in the follicle and thereby elevate the number of LH receptors.

The result of this is an increase in the LH stimulation of the oocyte and surrounding follicular cells. In the oocyte, LH works with FSH to induce disconnection between the oocyte and the cumulus oophorus, and decreases the level of cAMP to allow the reinitiation of meiosis. In follicular cells, LH (together with the pituitary hormone prolactin, in some animals) induces luteinization, which is expressed as in increased production of progesterone, prostaglandins $\mathrm{F}$ and oxytocin. Progesterone and prostaglandins $\mathrm{F}$ are necessary for the formation of an ovulatory stigma (a thinning of the wall of the ovary above the preovulatory follicle), which occurs through the local action of proteases (Fig. 1.8). They may also induce the constriction of smooth muscle fibers in the follicle, to assist the ovulatory expulsion of the follicular fluid and oocyte-cumulus complex. The pre-ovulatory surge of LH is eventually terminated by high concentrations of progesterone, possibly in association with a gonadotropin surge attenuating factor (GnSAF) also secreted by the ovary. Oxytocin starts to be secreted by the luteinizing follicle in some animals (ruminant artiodactyls) and is involved in the destruction of the corpus luteum if no pregnancy ensues (described below). In other animals, oxytocin from the ovary and the posterior pituitary gland may contribute to the induction of sexual behavior during the peri-ovulatory period, to ensure appropriately timed coitus fertilization of the ovulated egg. It may cause increased contractile activity in the oviduct, to facilitate transport of the fertilized egg to the uterus for implantation. 


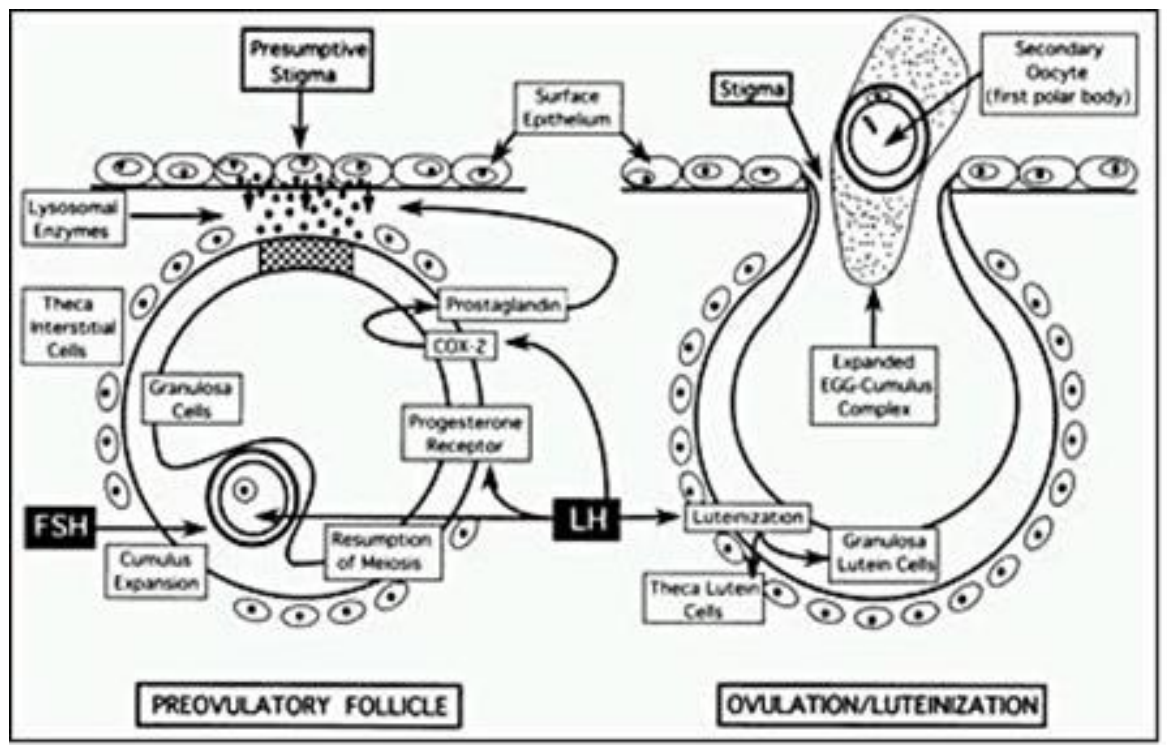

Figure 1.8: Cellular mechanisms by which the preovulatory surge of FSH and LH causes ovulation. (Erickson GF: The Ovary: Basic Principles and Concepts: In Felig P, Baxter JD, Broadus AE, Froman LA, (eds): Endocrinology and Metabolism, 3rd edition. New York, McGraw Hill, 1995)

\subsubsection{Regulation of luteogenesis and luteolysis}

The formation of the corpus luteum after ovulation is associated with a rapid rise in progesterone secretion by luteinised follicular cells. Most of this comes from the granulosa-derived large luteal cells and is unregulated, whilst a smaller amount comes from the theca-derived small luteal cells and is gonadotropin-regulated. Progesterone prevents the maturation and ovulation of other ovarian follicles, both by direct action and by inhibiting the secretion of high concentrations of FSH and LH. Progesterone also prepares the uterine endometrium for implantation, changing it from a proliferative state (brought about by preovulatory estrogens) to a secretory state. If the ovulated oocyte is fertilized and develops into a blastocyst (a very early embryo), progesterone facilitates its implantation. Under these circumstances, the lifespan of the corpus luteum is extended and the progesterone it secretes will continue to maintain the condition of the endometrium until this role is taken over by hormones from the placenta. If no implantation takes place (because the oocyte was unfertilized or because of an incompetent embryo), the corpus luteum regresses. 


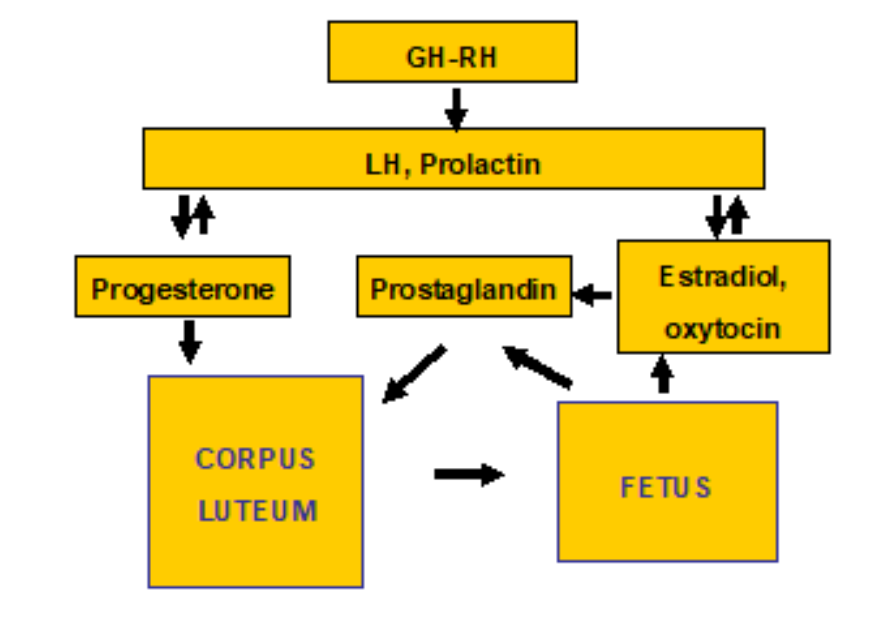

Figure 1.9: Regulators of the corpus luteum and pregnancy

The mechanism by which the corpus luteum is maintained in the presence of an implanted embryo (the "maternal recognition of pregnancy") or lost in an infertile cycle, varies between animals. It may require hormonal support to continue its activity in the event of pregnancy, or it may be capable of autonomous survival an require active termination if pregnancy does not ensue. In humans, the conceptus secretes a gonadotrophin-like hormone (human chorionic gonadotrophin, hCG) which directly stimulates and maintains the otherwise transient corpus luteum. It is this hormone which is detected in routine pregnancy tests, such as those based on a urine sample and dipstick. By contrast in ruminants, the corpus luteum of an infertile cycle is terminated by a positive feedback loop involving uterine prostaglandin F (the luteolytic agent) and luteal oxytocin (secreted by granulosaderive large cells); if implantation occurs, this loop is broken by interferons secreted by the conceptus, allowing the corpus luteum to survive. In pigs, prostaglandin $\mathrm{F}$ is similarly luteolytic, but in this species estrogen from the conceptuses alters the permeability of the endometrium and redirects the prostaglandin back into the uterine lumen so that it cannot enter the blood stream and is unable to reach the ovary. Other mechanisms operate in other animals and this is an aspect of comparative reproductive physiology with great variation and complexity.

\subsection{Artificial regulation of the ovarian cycle}

Biotechnologists have used their understanding of the reproductive process to control breeding in domestic animals so as to increase the numbers of offspring produced, maximize the availability of desirable genetic traits and increase productivity. It has also been possible to avoid problems in fertility, reduce wastage and minimize the need for veterinary intervention. In human reproductive 
science, the priorities have been to overcome infertility and reproductive disease whilst offering better and more diverse methods of contraception and approaches to family planning. In both animals and man, these biotechnologies have been extraordinarily successful and research continues to offer new and exciting possibilities.

Not all developments have been unequivocally beneficial, however. Artificial insemination and breed selection have been exploited, particularly in the cattle industry, for many years and have brought about large increases in milk yield and improvements to meat quality. Gains in milk yield may now have reached their maximum potential and there is increasing evidence that they may have been obtained at the expense of overall fertility, particularly in high producing breeds. Gene interactions, coupled with the inherent limits of metabolism, suggest that an undue focus on individual productive characteristics may not be the best way forward as far as domestic animals are concerned. Current research is looking at molecular approaches to genetic manipulation, including cloning, but the techniques available have yet to reach the level of widespread commercial application and are also subject to ethical resistance by some consumers.

All new methodologies continue to depend on a deep understanding of the reproductive process itself. Virtually all approaches, whether physiological, endocrinological, embryological or genetic, involve some manipulation of the underlying ovarian cycle. This is either to generate large numbers of germ cells for intervention and manipulation or to facilitate the timed development of offspring and the maximization of reproductive success. Two types of intervention, the synchronization of ovarian cycles and the induction of superovulation, are particularly valuable and have been used together in the large scale production of farm animals.

\subsubsection{Synchronization of the ovarian cycle and contraception}

The production of farm animals can be simpler and cheaper when the reproductive cycles of different animals are synchronized. Synchronization within the maternal herd allows the breeder to carry out artificial insemination in large number of animals at once and to choose the optimal time for producing offspring.

Synchronization of breeding can be achieved by temporarily stopping or abrogating cycles using blocking factors, and then allowing all animals to resume cyclicity in the same phase at a controlled moment. A range of physical and chemical interventions can be used to halt the cycle but hormonal substances or their pharmacological analogues, used in form of injections, intravaginal sponges or subcutaneous implants, are the most popular methods.

In some species (sheep, chicken), the reproductive cycle is regulated and synchronized by exposure to natural or artificial changes in photoperiod. Photoperiod can affect mammalian 
reproduction via the hormone melatonin, which is produced in the pineal gland (epiphysis cerebri) during the dark phase of the circadian cycle. The natural period of secretion of melatonin is extended during the longer nights of winter, and this change may encourage or inhibit reproductive activity according to species. In sheep, which naturally begin to breed as the nights lengthen in autumn, injections or bolus feeds of melatonin can be used both to synchronize estrous cycles within the flock and to advance or extend the reproductive season.

In some animals it is possible to achieve synchronization by exploiting behavioral stimuli. For example, the preovulatory gonadotropin surge and final stages of folliculo- and oogenesis, including ovulation, may be supported (pigs, birds) or induced (rabbits) by mechanical (coitus), visual (courtship, mating behavior) or chemical (pheromones) signals from the male. Controlled contact with a reproductively active male, or exposure to natural or artificial male pheromones, can be a very efficient means of cycle synchronization prior to timed natural mating or artificial insemination in these species. Changes in nutrition, temperature and other environmental factors have been applied to other domestic mammals and birds, but in general have been somewhat less successful.

As mentioned previously, folliculogenesis is blocked during the luteal phase of the ovarian cycle or during pregnancy by low circulating concentrations of gonadotropins and high rates of progesterone secretion by the corpus luteum. Blockade of the follicular phase can be achieved by preventing gonadotropin secretion, using pharmacological analogues of GnRH or hormones such as inhibin or by blocking gonadotrophin action by means of receptors antagonists. Injections and implants of progesterone or its synthetic analogues can mimic the hormonal environment of the luteal phase and inhibit folliculogenesis. Removal of the treatment allows cycles to be restarted simultaneously in different animals.

An alternative approach to synchronization is artificially to end the luteal phase of the cycle and allow follicular development to resume. This can be achieved by mechanical removal of corpora lutea (for example in cattle, by rectal manipulation) or by treatment with a luteolytic agent such as prostaglandin $\mathrm{F}$ or one of its analogues.

Treatment with progesterone or its analogues, to block folliculogenesis and prevent ovulation, provides the basis for most current chemical approaches to contraception for women. These methods are generally extremely safe and reliable and have the advantage of near instant reversibility. There have been successful attempts to achieve a more permanent inhibition of folliculo- and oogenesis by immunoneutralization of gonadotropins or ovarian components (for example, of the proteins in the zona pellucida etc.), although these methods have yet to be widely exploited.

There are reports that prolactin, a pituitary hormone normally associated with lactation but which also inhibits folliculogenesis, can be used to maintain a luteal phase-like state and thereby act as a 
contraceptive. An understanding of this aspect of prolactin function has come from investigations into a type of amenhorrhoea associated with hyperprolactinemia and from an endocrinological explanation of the reduction in fertility which may occur during human lactation.

\subsubsection{Induction of superovulation}

Induction of superovulation is a biotechnological method to stimulate the development and ovulation of multiple ovarian follicles, thereby increasing the number of offspring or providing numerous germ cells for laboratory manipulation or research. This method is used in animal breeding to maximize the production of genetically valuable organisms and in human and veterinary medicine for the treatment of ovarian insufficiency and infertility. Superovulation can be used in combination with other methods (cycle synchronization, oocyte IVM/IVF, embryo transfer etc.) to increase the number of offspring in a short period of time.

To induce superovulation, the natural physiological signals which induce folliculogenesis, oogenesis and ovulation (see above) are promoted or mimicked. The majority of protocols for superovulation employ hormones such as $\mathrm{GnRH}$ or gonadotropins (in recombinant synthetic form or as a biological extract), injected at the start of the natural follicular phase or after artificial termination of the luteal phase by means described above. The following substances are currently used for the induction of superovulation:

FSH or PMSG (pregnant mare serum gonadotropin, a natural FSH analogue) injected during or instead of the natural peak of FSH release.

$>$ LH or hCG injected during or instead of the natural peak of LH release, which occurs after FSH peak.

$>$ Synthetic GnRH and its agonistic chemical analogues, sometimes injected together with gonadotropins to promote the release of endogenous gonadotropins.

$>$ Immunoneutralisers of inhibitors of gonadotropins (inhibin etc.) to promote endogenous gonadotropin release.

Gonadotropin-induced superovulation can be made more efficient by additional treatment with extracellular mediators of gonadotropin action on the ovary, such as IGF-I, its analogues or its endogenous stimulator $(\mathrm{GH})$. Treatments with pharmacological regulators of cAMP and protein kinase A, which are intracellular mediators of hormone action on ovarian cells, may promote natural and hormone-induced ovulation. In some species environmental factors (photoperiod and mating in birds, coitus in mammals with induced ovulation) are useful for superovulation as well as for the timed induction of ovulation. 
At present, combinations of FSH and LH or their analogues, are considered the most efficient inducers of superovulation in domestic species. Nevertheless, current methods for both ovarian cycle synchronization and induction of superovulation have serious disadvantages. Modern large-scale application of these methods does not allow for the adequate monitoring of ovarian cycles in all treated animals, or make allowance for individual variation in the timing and length of cycles, in the patterns of release of endogenous hormones, or in the sensitivity of target tissues to both endogenous and exogenous hormones. Inadequate application of treatment results in a low number of ovulated eggs capable of fertilization and further development. It may also result in the formation of follicular and luteal ovarian cysts, to insensitivity to further hormonal stimulation and to infertility. Only a relatively limited number of substances are available for the physiological control of ovarian cycles, and even fewer are currently used routinely in agriculture and medicine. Further detailed research is required in this area.

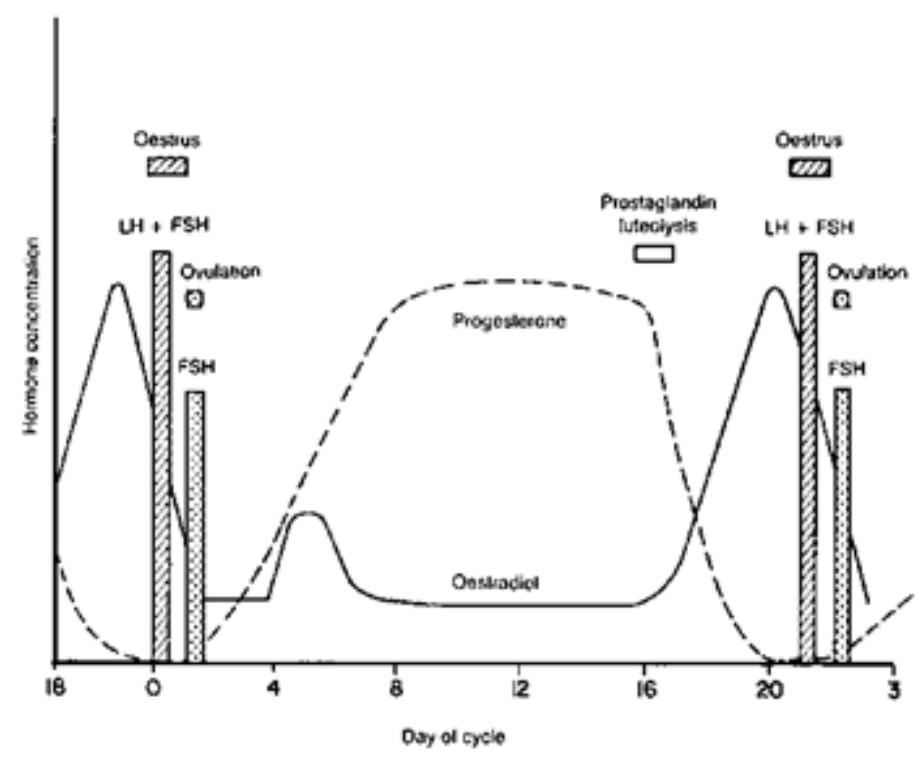

Figure 1.10: The changes in plasma hormone concentrations during the bovine natural and induced oestrous cycle. Day $O=$ day cow first shows estrus (Peters $A R$ and Lamming $G$ E. Hormone patterns and reproduction in cattle. In Practice 5: 153-157, 1983)
Abbreviations
BMPs - bone morphogenetic proteins
cAMP - cyclic adenosine monophosphate
CDC2 - cyclin dependent kinase
$\mathrm{FSH}$ - follicle stimulating hormone
$\mathrm{GnRH}$ - gonadotropin releasing hormone 
GVBD - germinal vesicle breakdown

IGFBPs - IGF binding proteins

IGFs - insulin-like growth factors

LH - luteinizing hormone

MAPK - mitogen-activated protein kinase

MAS - meiosis activating sterol

$\mathrm{MPF}$ - maturation or M-phase promoting factor

OMI - oocyte maturation (or meiosis) inhibitor

PKA - cAMP-dependent protein kinase A 



\section{Quality of mammalian preimplantation embryos and its evaluation}

\subsection{Introduction}

Embryo morphology assessment, however imperfect it may be, is at present the most popular method for embryo selection prior to transfer, both in human and bovine assisted reproduction. The first embryo transfers in cattle were performed with in vivo produced embryos. Following superovulation and artificial insemination, bovine embryos are flushed out the uterus at day 7 of the oestrous cycle. These embryos are predominantly at the morula stage, whereas only a small percentage is represented by blastocysts. A major difference between human and bovine embryos is the fact that in the latter, assessment of morphology is jeopardized by the opacity of the blastomeres, which is caused by lipid droplet accumulation. This opacity makes it difficult to assess nuclear and nucleolar morphology, aspects which can easily be evaluated in human zygotes or early cleaving embryos. However, recent research which focused on correlation between bovine embryo morphology and embryonic ultrastructure, gene expression and cryoresistance, has provided evidence that much more can be deduced from mere embryo morphology than previously thought. Morphological features such as colour of the blastomeres, the extent of compaction, timing of blastocyst formation and expansion and diameter of the embryo at hatching can be linked with embryo quality. On the other hand, cattle embryos of deviant chromosomal constitution or with aberrant genetic make-up cannot be selected by means of the current morphological techniques. Possible solutions include the visualization of bovine pronuclei at the zygote stage by means of ultracentrifugation or multiphoton laser scanning microscopy, and adjustment of genetic analysis in order to reconstruct embryo genetic make-up starting from the biopsy material.

The two most useful predictors of viability of preimplantation embryos are (i) normal morphology, and (ii) fast cleavage rate. Another approach is the study of chemical messages released by the egg (early pregnancy factors [EPF], cytokines and other luteotrophic factors). These specific factors influence ovum transport and ovarian activity in vivo. To increase embryonic quality in vitro, several co-culture systems have recently been designed, which allow a selection in vitro of the best embryos. It is not easy to determine the future of the embryo in vivo as genital tract secretions may interfere positively, but also negatively with embryo development and implantation.

Another important variable, that is too often overlooked, is the embryo transfer technique itself. A smooth and cautious transfer with no trauma to the endometrial lining is essential to give the embryos the best chance for continuing with normal development. Embryo quality, to a great extent, is determined by the quality of the egg from which it started. Tests of ovarian reserve, such as the day 
3 FSH hormone assessment, and other tests - can give us additional information about egg (and embryo) quality.

\subsection{Developmental processes in embryos}

After a successful contact between the oocyte and spermatozoa, a subsequent zygote will undergo a number of cleavages during the first 5 days of development. At 5 days post fertilization the embryo starts to compact. Compaction is a developmental process which is one of the morphological parameters important in the assessment of embryo quality.

\subsubsection{Compaction}

The process of embryo compaction starts at day 5-6 post fertilization in the cattle with the formation of tight junctions between blastomeres (Fig. 3.1.). The formation of tight junctions, along with shrinkage of blastomeres, results in a rounder and more compact embryo with a larger perivitelline space. Morphologically this means that it becomes more difficult to see individual cells and the embryo resembles a tight clump of cells. Under some IVP (in vitro production) conditions, this compaction process is less obvious. However, when in vitro fertilized zygotes are cultured in host oviducts (e.g. sheep), compaction is normal. During the process of compaction, the embryo is called a morula or early morula, whereas following compaction it is known as a tight morula. This process of compaction normally starts around the 32-cell stage in the bovine both in vivo and in vitro embryos.

\subsubsection{Blastulation}

As a result of the formation of tight junctions, the embryo forms inner and outer cell types. This is a prerequisite for the process of blastocoel formation, which takes place at day 6-7 post fertilization in the cow (Fig. 3.1.). In the sheep and pig, blastocysts appear at 4-5 days and 3-5 days post fertilization respectively. Due to the active transport of sodium passing through the outer cell layer, anion gradient is formed, leading to the osmotic accumulation of fluid. This results in the formation of the blastocoel cavity just beneath the outer layer of cells.

At the early blastocyst stage, one can distinguish two types of blastomeres: differentiated trophoblast cells, lining the blastocoel, and undifferentiated inner cells (Fig. 3.2.). Blastocoel formation normally starts on one side of the embryo and the resulting developmental stage is known as an early blastocyst (Fig. 3.2A.). The blastocoel occupies less than $50 \%$ of the total embryo in early blastocysts. When the process of blastocoel formation continues, the inner cells/blastocoel ratio 
changes further. When the blastocoel occupies more than $50 \%$ of the embryo, the embryo is classified as a blastocyst or mid-blastocyst.

As the osmotic pressure inside the blastocoel cavity rises, the embryo starts to expand and the overall diameter of the embryo increases. At the same time, the zona pellucida stretches and becomes thinner. At this stage the embryo is classified as an expanded blastocyst (Fig. 3.2B.). One of the most striking characteristics of an expanded blastocyst is the inner cell mass (ICM). It is a tightly compacted, spherical clump of cells attached to the wall of the trophoblastic vesicle. The number of ICM cells may differ between in vivo-derived and embryos produced in vitro in different culture systems. During the expanded stage, which persists for one or two days, the trophoblast may sometimes collapse. One of the reasons for such a collapse may be hypothesized an imperfect cell division (formation of tight junctions).

\subsubsection{Hatching}

At day 9-11 post fertilization, the zona pellucida ruptures and the embryo hatches. This process is probably caused by increased pressure of the embryo within the zona, eventually combined with cycles of expansion and collapse or an enzymatic process initiated by the trophoblast. When the process of hatching is completed in vitro, the embryo is often pear-shaped or collapsed (Fig.3.1.). Later the embryo increases in size and regains its spherical form (Fig. 3.2C). At this stage, there is a large increase in the number of trophoblast cells and the embryo starts elongating (Fig. 3.3). This phenomenon is not reproducible in vitro. 


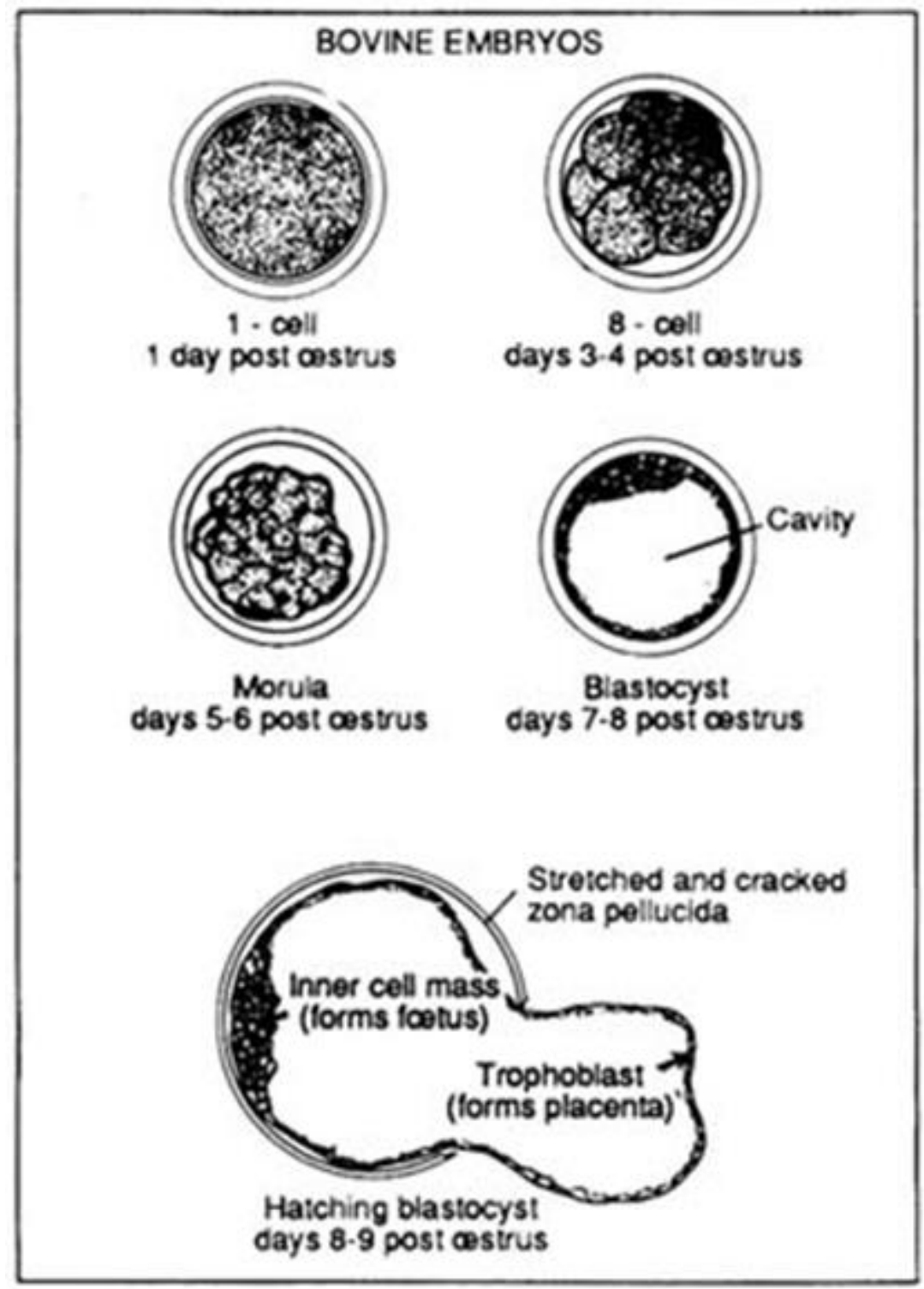

Figure 3.1: Preimplantation stages of bovine embryo development 

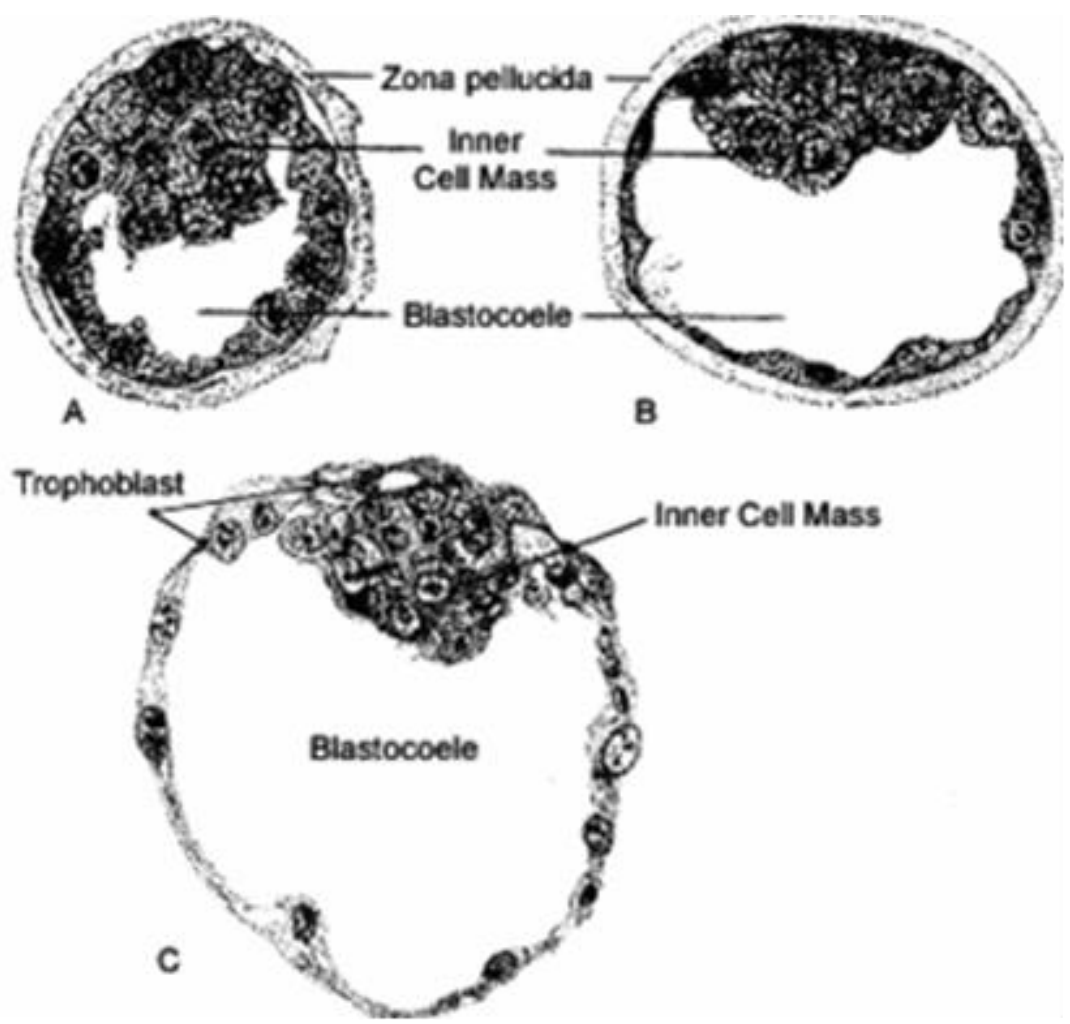

Figure 3.2: Early (A), expanded $(B)$ and hatched $(C)$ bovine blastocysts

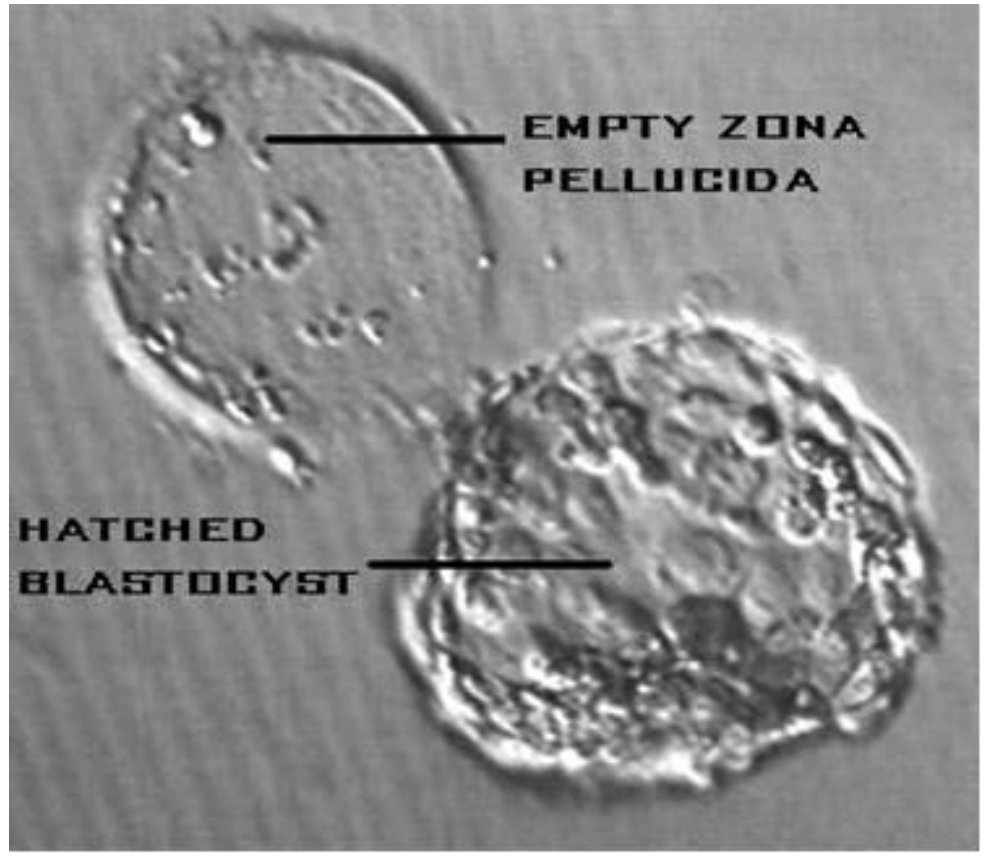

Figure 3.3: Morphology of hatched bovine blastocyst 


\subsection{Classification of embryonic stages in cattle embryos.}

The coding for embryonic stages in cattle embryos is shown in Table 3.1. This scoring system differs from the IETS code in the last two stages. Whereas IETS describes stage 8 and 9 as hatched or expanded hatched, this system discriminates a hatching or hatched blastocyst by stage 8 and 9 respectively.

Table 3.1: Coding for embryonic stages in cattle embryos.

\begin{tabular}{|c|c|l||}
\hline Code & Stage & \multicolumn{1}{c||}{ Morphological description } \\
\hline \hline 3 & Early morula & A clump of 16-32 non-compact larger blastomeres \\
\hline 4 & Morula & A clump of 32-64 compact smaller blastomeres \\
\hline 5 & Early blastocyst & Blastocoel <50\% \\
\hline 6 & Blastocyst & Blastocoel $>50 \%$, ICM and TE discernible \\
\hline 7 & Expanded blastocyst & $\begin{array}{l}\text { Overall diameter of embryo increases, zona pellucida becomes } \\
\text { thinner }\end{array}$ \\
\hline \hline 8 & Hatching blastocyst & Rupture of zona pellucida, partially hatched embryo \\
\hline \hline 9 & Hatched blastocyst & $\begin{array}{l}\text { Fully hatched embryo increasing in size, empty zona pellucida } \\
\text { with some extruded cells left }\end{array}$ \\
\hline
\end{tabular}

\subsubsection{Classification of embryos by quality grade}

The assessment of the embryo is based on a number of morphological parameters, which might interfere with embryo quality. Classification of embryos into different morphological grades is performed by means of a stereomicroscope. Different aspects of the morphological appearance are evaluated to give the embryo its final grade.

1). Presence or absence of cellular fragments.

Due to the prominent perivitelline space, the number of extruded cells or the amount of cellular debris can easily be assessed in bovine and human embryos (Fig. 3.4A-F.). For accurate assessment it is important to roll over embryos under the microscope in order to visualize them in a three-dimensional perspective. In later developmental stages extruded cells are forced against the zona pellucida and are often difficult to distinguish. With appopriate magnification and careful observation however, extruded cells can often be detected, although the counting of debris in expanded blastocysts remains very difficult. 


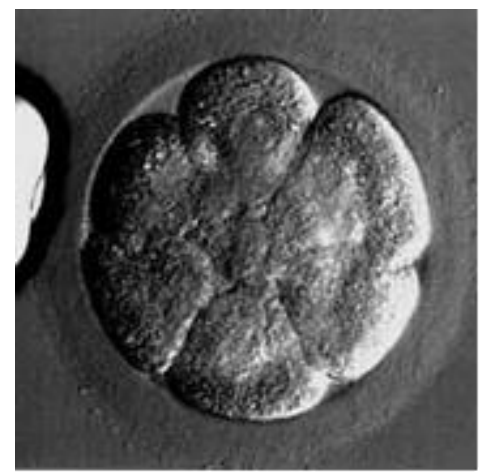

Figure 3.4A-F. Grading of embryos on the basis of presence of cellular fragments

A: This is a perfect 8-cell embryo (day3). There is no fragmentation and the cells are very even, regular, and similarly sized. 8 cell, grade 4.

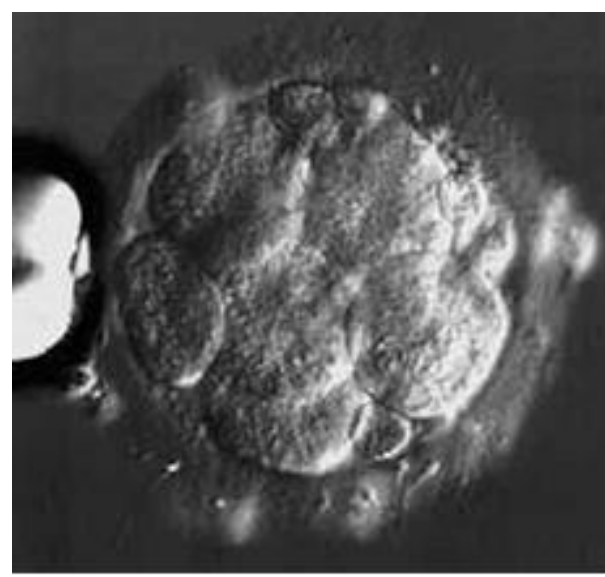

B: A very slightly fragmented embryo is being performed. The holding pipette is on the far left. A sperm is clearly visible at 7 o'clock (this one lost the race) 8 cell, grade 3

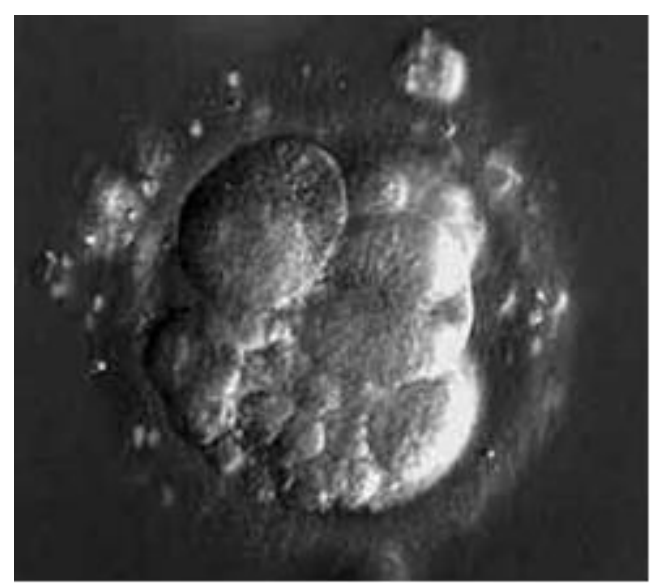

C: This embryo is moderately fragmented and has unevenly sized cells (blastomeres) 5 cell, grade 2 


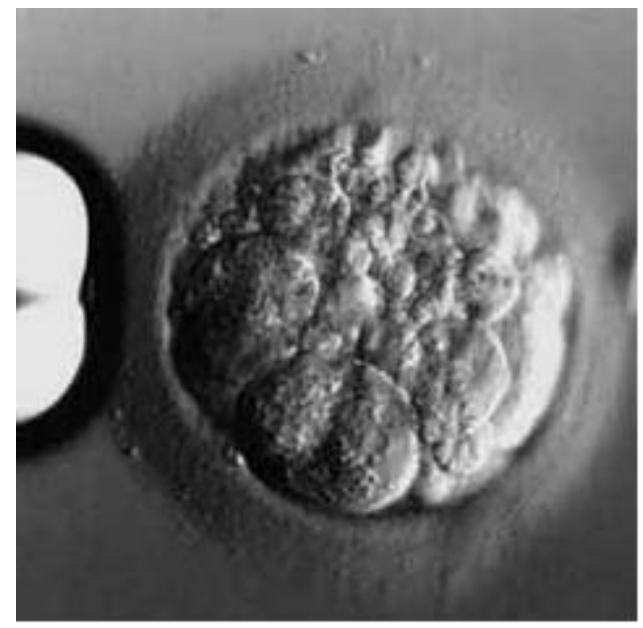

D: This embryo is severely fragmented and also has unevenly sized cells (blastomeres) 4 cell, grade 1 .

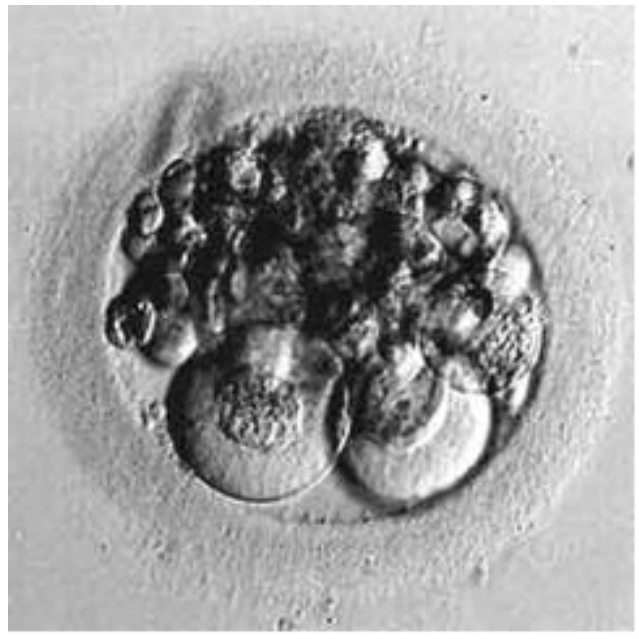

E: This embryo is also a day 3 embryo. It is severely fragmented and very low quality. 2 cell, grade 0 .

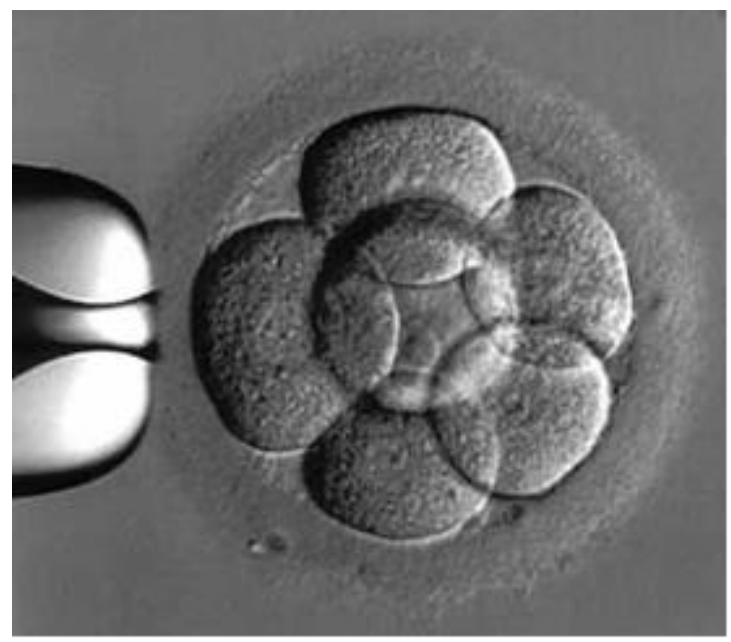

F: A high duality day3 human embryo at the 8-cell stage. 6 cells are visible in this plane of focus. 
2). The degree of compaction.

Compaction can be assessed by the roundness and/or the sharpness of the contours of the outer periphery of the morula. The sharpness of the inner border of the ICM can be visualized by rolling the embryos with a pipette.

Besides the degree of compactness, also the size of the ICM (normal, small, absent), is taken into account in the evaluation of embryo quality. In vitro, the number of cells allocated to the ICM varies enormously, sometimes resulting in embryos which resemble „empty“ trophoblastic vesicle.

\section{3). Colour and texture of blastomeres.}

The colour of an embryo or ICM, which is partially influenced by the amount of transmitted light under the microscope, ranges from light to dark and is dependent on a number of factors:

a). The uniform distribution of lipid vesicles and cytoplasmic organelles;

b). The amount and size of lipid vesicles in the cytoplasm;

c). The number of pycnotic cells;

d). The total number of blastomeres;

e). The source of the embryo (in vivo versus in vitro);

f). The nutrition of the donor cows (for in vivo embryos) and embryo culture conditions (for IVP embryos).

It is known that IVP embryos have a darker texture than in vivo embryos. It has been suggested that the degree of darkness of the embryo and its granulation depends on the source of serum which has been used for embryo culture. These granules are particularly visible at the blastocyst stage in the trophectoderm cells. Serum-free embryo culture conditions produce much lighter embryos; although some batches of bovine serum albumine (BSA) can also lead to dark embryos. For in vivo produced cattle embryos, a significant correlation between embryo colour and donor blood cholesterol has been shown. Moreover, cattle embryos from high-yielding dairy cows often exhibit a darker texture of the blastomeres than embryos from beef cattle.

\section{4). Uneven cleavage divisions}

Asynchronous cell division may occur at any time after the first cell cycle. Thus, three, five, six and seven cell stage embryos are not uncommon. As a consequence, especially at the morula stage, cell size may affect a symmetry and spherical appearance of the embryo and may be related to embryo quality. 
Evaluation of embryo quality grade is important to predict pregnancy rates after transfer and to select embryos for cryopreservation. In 1983, Lindner and Wright proposed a useful and practical system for scoring day 7 bovine embryos. This system combines a number of developmental and morphological criteria to classify embryos as excellent (I), good (II), fair (III) or poor (IV). A more simplified version of the Lindner and Wright scoring system has been published by the International Embryo Transfer Society (IETS). It combines embryos of grades I and II into the IETS grade I, whereas IETS grade 2 embryos are of fair and IETS grade 3 are of poor quality (IETS Manual, 1998). The IETS scoring system has become the standard reference for embryos that are exported/imported internationally. In the latest IETS manual (third edition, 1998), the codes for embryo quality are described as follows:

\section{Code 1: Excellent or good}

Symmetrical and spherical embryo mass with individual blastomeres, which that are uniform in size, colour and density. This embryo is consistent with its expected stage of development. At least $85 \%$ of the cellular material should be an intact, viable embryonic mass. The judgement should be based on the percentage of embryonic cells extruded into the perivitelline space. The zona pellucida should be smooth and have no concave or flat surfaces that might cause the embryo to adhere to a Petri dish or a straw.

Code 2: Fair

Moderate irregularities in overall shape of the embryonic mass or in size, colour and density of individual cells. At least $50 \%$ of the cellular material should be an intact, viable embryonic mass.

Code 3: Poor

Major irregularities in shape of the embryonic mass or in size, colour and density of individual cells. At least $25 \%$ of the cellular material should be an intact, viable embryonic mass.

Code 4: Dead or degenerating

Degenerating embryos, oocytes or 1-cell embryos, non-viable.

\subsection{Techniques of blastocyst quality evaluation}

It is important to choose a proper technique for evaluation of blastocyst quality (Tab. 2.). As far as selected embryo is to be transferred into uterus, non-invasive techniques should be used. Though, invasive methods provide more exact information about embryo viability. At the evaluation of 
survival of early preimplantation embryos, most used criteria are morphological, from which a total cell number of the embryo is a more proper indicator of quality. Less invasive parameter for testing of embryos survival is showed to be a diameter of estimated embryos.

Table 3.2:Definition of non-invasive and invasive techniques for embryo quality evaluation

\begin{tabular}{|l|l||}
\hline Non-invasive techniques & Invasive techniques \\
\hline Morphology of embryos & Cytogenetic analysis \\
\hline Dynamics of development & Cell number \\
\hline \hline Metabolic tests & Differential staining \\
\hline \hline Formation and hatching of the blastocyst & TUNEL technique \\
\hline \hline Vital staining & Cellular components \\
\hline \hline Cryoresistance & Utrastructural analysis \\
\hline \hline
\end{tabular}

\subsubsection{Non-invasive techniques}

More currently used, although very subjective method of embryo selection, is an evaluation of embryos from morphological point of view. This method is widely used for choosing embryos for the transfer; however the results are influenced by subjectivism of the researcher, especially with in vitro produced embryos. The reason of this subjectivity is that the compaction of in vitro produced embryos of cattle is not enough visible, what narrows perivitelline space and complicates to identify a fragmentation. In human embryos, a morphology at Day 3 has no a predictive value for blastocyst development, however worse morphology at blastocyst stage is related to decreased hCG production.

In general, the way we assess the "quality" of embryos from in vitro fertilization is by determining 3 major components. Cell number, cell regularity (regularity of size), and degree of fragmentation. There are also other things that are also noted about the embryos appearance, such as multinucleation, presence of vacuoles, granularity, thickness of the shell around the embryo, etc.

Usually, determinations of "quality" are not made until about 48 hours (or later) after the egg retrieval. By 48 hours ("day 2"), we prefer that at least some of the embryos are at least 3 cells - and preferably 4 cells or more. They must be at least 2 cells by then - or they have basically "arrested". By 72 hours ("day 3"), we prefer that some of the embryos are at least 6 cells - and preferably at least a few that have 7 cells or more. Embryos with higher cell numbers and regular appearing cells (blastomeres) and little or no fragmentation have a higher overall chance of implanting than do their counterparts with less cells, more irregularity and more fragmentation. 
Embryo quality as we see it under the microscope in the IVF lab gives us some reasonable ability to predict the chances for pregnancy from an embryo transfer. However, because there are many other contributing factors involved that we can not measure, these generalizations do not always apply. We see some cycles fail after transferring 3 perfect looking embryos, and we also see beautiful babies born after transferring low grade embryos. The true genetic potential of the embryo to continue development and the quality and receptivity of the uterine lining are really impossible to measure. Hopefully, that will be something for the future.

Other method for selection of developmentally proper embryos is developmental dynamics. Dynamics of blastocyst formation is related to cell number and resistance to freezing. Fast cleaving in vitro produced embryos reached morulae and blastocysts at significantly higher frequencies than slower cleaving embryos. In addition, the faster cleaving embryos showed a lower incidence of fragmentation. The timing of blastocyst development was correlated with cell numbers in the blastocysts: there were significantly fewer total and ICM cells in Day 8 versus Day 6 or Day 7 blastocysts.

Metabolic tests for evaluation of embryo survival involve measurements of nutritive absorption and energy metabolism, oxygen absorption, production of enzymes, hormones or growth factors. Hatching of blastocysts is generally considered as a final point, because the embryo needs to be released from the zona pellucida. However, there are significant differences between in vivo and in vitro embryos in terms of blastocyst hatching from the zona. Particularly, hatching of blastocysts in in vitro embryos of cattle is associated with gradual expansion and subsequent thinning of the zona pellucida, whilst in in vitro embryos such expansion stage is not expressed.

Vital staining and resistance against freezing belong to techniques, which may partially compromise the embryo. At vital staining membrane integrity, which is critical for embryo survival, is estimated on the basis of the presence of fluorescent dye (Fig. 3.5.) Double staining techniques, such as SYBR/ propidium iodide or Hoechst 33332/ propidium iodide were also used for the evaluation of sperm viability. This nuclear dye either is able (SYBR 14, Hoechst 33332) or unable (propidium iodide) enter across intact membrane. These properties can be used at the estimation of embryo survival. Test of freezing also may be used for embryo quality estimation. Differences in sensitivity to cooling in in vivo and in vitro produced embryos of cattle may be related to differences in membrane composition. 


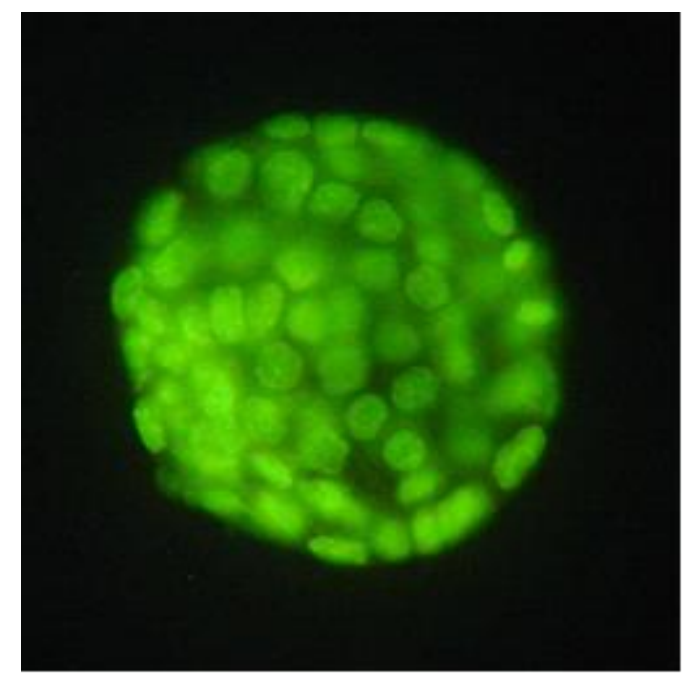

Figure 3.5: Staining of embryonal nuclei by the fluorescent dye SYBR 14

Due to a lack of alternative non-invasive methods, embryo quality estimation based on morphological parameters is still the most commonly used method. Advantages of this metod are the limited time needed per embryo and the ease of learning it. A disadvantage however, especially with in vitro produced embryo, is that embryo viability, based on pregnancy rate, still varies within groups of embryos with the same grade. Therefore, a combination of embryo morphology and other noninvasive methods e.g. timing of development or quick metabolic tests would bring more objective information about the embryo quality.

The ultimate test of the developmental potential of the embryo after its transfer into a suitable recipient is a birth of normal young animal. The ability of the embryo to survive stresses due to freezing can be considered as an initial "screening test". Embryos that have been derived in vivo, are usually selected on the basis of their morphology. Generally, there appears to be a good association between embryo "quality", assigned on the basis of morphology, and the likelihood of pregnancy. For example, although assigning a "grade" to an embryo is a rather subjective judgement, embryos can be assigned numerical grades on the basis of their morphology. In general, poorer quality embryos are less likely to yield a pregnancy, whilst better quality embryos are more likely.

Unfortunately, embryos produced in vitro are less viable than their in vivo-derived counterparts, and their cryopreservation is often less successful. Initially, the objective of in vitro embryo production was simply to increase the number of blastocysts with relatively little attention to their quality. It soon became evident, that embryo quality was of primary importance for success of micromanipulation of bovine embryos. Differences between two sorts of embryos (in vivo and in vitro) become most evident when they are subjected to various stresses, although reasons for these differences have not really been explained. 
When produced in the presence of serum, in vitro-produced embryos appear darker than their in vitro-derived counterparts, and have a much smaller perivitelline space, suggesting that compaction of adjoining cells within in vitro-produced embryos is less dense. It is supposed, that the darker appearance and more diffuse packing of embryos reflect differences in lipid content and composition of the two categories of embryos.

\subsubsection{Invasive techniques}

Invasive evaluation of embryo quality involves procedures of fixation and staining of embryos. Using this approach it is possible to determine chromosomal status, nuclear, mitochondrial status, cytoskeleton and state of cell organelles of the embryo.

Cytogenetic analysis of embryos is very important, because in vitro culture may cause chromosomal abnormalities. Karyotype of in vitro produced blastocysts showed chromosomal abnormalities in $44 \%$ of embryos, whilst at in vivo fertilization - only in $20 \%$ of embryos. For evaluation of chromosomal abnormalities in developing bovine embryos on Day 7 or 8 following in vitro or in vivo fertilization a fluorescent in situ hybridization (FISH) using specific fluoroprobes to 6 th or 7 th chromosomes can be used. This approach enabled to find that $72 \%$ of in vitro produced blastocyst were mixoploid, i.e a mixture of normal, diploid or polyploid cells, comparing to significantly lower rate of mixoploidy $(25 \%)$ at in vivo derived blastocysts.

At determining nuclear status major impact should be given on apoptotic index, i.e. proportion of apoptotic (death) cells to total cell number of the embryo. In addition to cell division and differentiation within the preimplantation embryo, it is becoming apparent that cell death by apoptosis is common even in vivo, with the majority of blastocysts having one or more dying cells. The percentage of dying cells within a blastocyst is about 10-20\%. The onset of apoptosis in the mammalian blastocyst coincides with activation of the embryonic genome. Apoptotic cells are characterized by blebbing of the nuclear membrane, chromatin condensation, cytoplasmic vacuoles and the formation of both nuclear and cytoplasmic fragments. Apoptosis is an essential feature of many normal processes and pathological conditions and serves a variety of purposes, including tissue homeostasis and remodeling, and the removal of unwanted cells. The role of apoptosis at preimplantation stages remains unknown. The presence of apoptotic cells in vivo suggests about its developmental role, which has not yet been elucidated. It is possible that the higher levels of apoptosis in the ICM of mouse blastocysts are to remove ICM cells which could potentially form TE. In addition, levels of apoptosis may be modulated by environmental factors. Apoptosis in preimplantation embryos is increased in suboptimal culture media in the presence of high concentrations of glucose and in the absence of specific growth factors. Assesment of apoptosis is 
also useful in evaluating the impact of a variety of pathological conditions and toxic insults on preimplantation development. Such studies include examining the effect of diabetes on preimplantation development in rat and mouse and growth factor modulation of UV irradiation damages in rabbit embryos.

Mitochondrial status is evaluated either using fluorescent staining combined with confocal scanning (invasive technique) or more widely used double-photon microscopy, what belongs rather to non-invasive technique. Distribution of cytoskeletal structures i.e. actin and tubulin may be visualized by fluorescent staining using confocal scanning.

Differences in embryo quality can be also determined by evaluation of ultrastructure. Main difference is reflected in cell organelles of morula and blastocysts, as for instance, lower number of lyzosomal vesicles and higher number of lipid droplets in embryos developed in serum-contained medium.

Either invasive or non-invasive techniques however have certain restrictions. If blastocyst is evaluated on the basis of differentiation of totipotent cells on ICM and TE, a differential staining is a method of choice. More data about the embryo quality can be provided by total cell number and distribution of embryo cells in the inner cell mass (ICM) or trophectoderm (TE) area determined after differential staining (Fig. 3.6.). The inner cells and outer cells of the blastocyst are the inner cell mass and the trophectoderm respectively. Therefore, the ratio of the number of inner and outer cells is a tool to determine this ratio. If the pattern of allocation of inner and outer cells is disturbed, it often leads to non-viable embryos. In in vivo grown embryos the ratio varies between $30-40 \%$ for different species studied so far (Table 3.3.).

Table 3.3: Overview of appearance of inner cells and ICM/total cell ratio in different species.

\begin{tabular}{|c||c||c||l||}
\hline Species & $\begin{array}{c}\text { First appearance of } \\
\text { inner cells }\end{array}$ & $\begin{array}{c}\text { ICM/Total cell ratio in } \\
\text { early to mid blastocyst, } \%\end{array}$ & \multicolumn{1}{|c|}{ Reference } \\
\hline \hline Laboratory & & & \\
\hline \hline Mouse & $13-16$ cells & 40 & $\begin{array}{l}\text { Handyside and Hunter, } \\
\text { 1984; Boerjan and te } \\
\text { Kronnie, 1993 }\end{array}$ \\
\hline \hline Rat & $11-23$ cells & $28-32$ & Pampfer et al., 1990 \\
\hline \hline Rabbit & Blastocyst & $21-30$ & Giles and Foote, 1995 \\
\hline \hline Bomestic & & 36 & Van Soom et al., 1996 \\
\hline \hline Sheep & $10-32$ cells & 44 & Handyside et al., 1987 \\
\hline \hline Porcine & $9-12$ cells & $22-25$ & Boerjan and teKronnie, \\
\hline \hline Human & & & Hardy et al., 1989 \\
\hline \hline
\end{tabular}


It has been shown that in different species allocation of inner cells can take place during a few consecutive cell cycles: in mouse inner cells are segregated predominantly during the fourth cleavage division, whilst in porcine embryos inner cells are segregated during transition from the 4- to 8-cell stage and inner cells are added during two subsequent cell cycles.

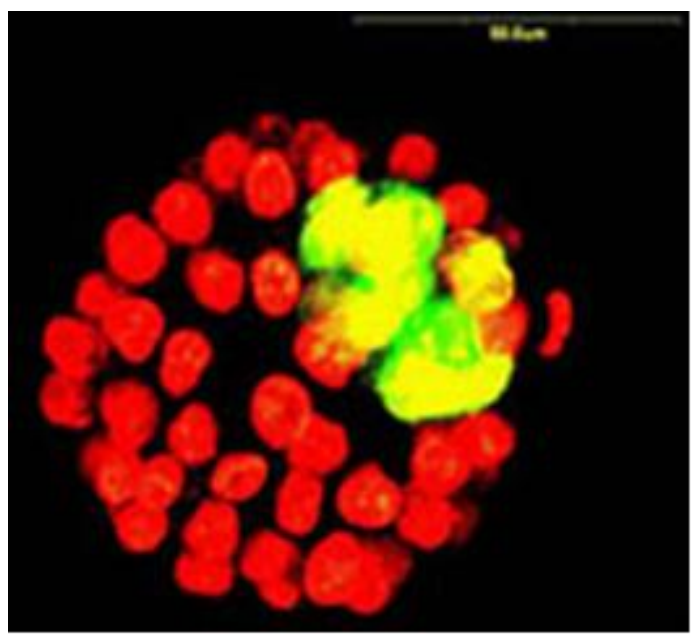

Figure 3.6: Differential staining of mouse blastocyst

\subsubsection{Techniques for differential staining}

As the outer cells of the blastocyst form a seal of zonular tight junctions, a quick procedure to differentiate between ICM and TE cells was developed using an immunological approach (immunosurgery). Although immunosurgery can be performed successfully on zona-intact mouse embryos, it is essential to remove the zona in embryos of most other species, since partial removal of the zona in porcine, bovine and human embryos results in incomplete lysis of outer cells. Chemical (acidic Tyrode solution) or enzymatic (pronase) treatments can both be used to remove the zona. An alternative is tearing the zona by means of needles or micromanipulation. It is also possible to use hatched blastocysts.

Immunosurgery is not always necessary to obtain differential staining. Trophectoderm cells can be permeabilized by chemical treatment with Ca 2+ ionophore A23187. This method was successfully used for differential staining of TE and ICM of porcine, murine and bovine embryos. Cells show an osmotic response to $\mathrm{Ca} 2+$ ionophore treatment, leading to cell lysis, which is visualized by vesiculation of the cell membrane. The conventional fluorochrom combination Hoechst 33342 or DAPI to stain intact ICM-cells and propidium iodide for lysed TE cells, cannot be used to evaluate with most laser scanning confocal microscopes, as it do not have a blue filter for DAPI staining. This 
problem can be solved using SYTO 17, a red vital nucleic acid stain in combination with SYTOX green stain.

Another adaptation of differential staining is a labelling the outer cells of zona-free blastocyst with FITC-labelled wheat germ agglutinin (WGA). WGA is a lectin which can be used as a cell surface probe to bind to sacharide residues on the plasma membrane of TE cells. After fixing, permeabilizing and staining with propidium iodide, TE cells fluoresce with green cytoplasm and red nuclei, while ICM cells only show red nuclei.

Because differential staining can be used in many species, it allows a good comparison of ICM development in different mammalian species under different culture conditions and to control for deviations in development due to micromanipulation, environmental influences or genetic make-up. Overall, reliable differential cell counts can be expected in approximately $85 \%$ of the embryos assessed.

\section{Abreviations}

BSA - bovine serum albumin

FISH - fluorescent in situ hybridization

hCG - human chorionic gonadotropin

ICM - inner cell mass

IETS - International Embryo Transfer Association

IVP - in vitro embryo production

TE - trophectoderm 


\section{In vitro embryo production}

\subsection{Introduction}

4.1.1 The history of in vitro embryo production.

The term in vitro literally means "in glass"; the fertilization takes place outside the body in a "glass" Petri dish (IVF). The term is used in contrast to in vivo "in the living body" fertilization.

Early attempts at IVF were made over a century ago, with successful rabbit embryo transfer carried out by Walter Heape in England. Gregory Pincus (1903-1967) and Enzmann performed further IVF experiments in the 1930s. An editorial in the New England Journal of Medicine in 1937 suggested IVF as a treatment for infertility in women. Although human IVF experiments were carried out in the 1940s and 1950s by John Rock, Menkin, and Landrum Shettles, knowledge of reproductive physiology was too limited for the IVF procedures to be successful. Then it was discovered that sperm had to undergo capacitation changes in the plasma membrane after ejaculation in order to be capable of fertilization, and that the oocyte (egg) also is not ready for fertilization until just before ovulation occurs. Armed with this knowledge, researchers successfully fertilized rabbit oocytes in vitro. In 1935, Gregory Pincus had claimed to have achieved successful mammal birth from the result of in vitro fertilization of rabbit eggs. As nobody, including Chang, could repeat this feat at the time, doubts were cast over the authenticity of the claim. Then finally, in 1959, Chang in vitro fertilized a black rabbit's eggs with a black rabbit's sperm, transferred them to a white rabbit, and was able to produce a litter of young black rabbits. This was the sort of evidence attesting to the feasibility of in vitro fertilization for which many scientists had been searching. IVF for many other species followed. The first baby conceived by in-vitro fertilization was born in England in 1978. Louise Brown - the first human baby conceived outside the woman was born. Since then, IVF has become a widely used method of infertility treatment, offered by hundreds of medical centers around the world.

\subsubsection{Embryo production in vitro}

In vitro embryo production (IVP - fig. 4.1) places major demands on oocytes and embryos with regard to developmental transitions, i.e. oocyte maturation, fertilization, compaction, blastocyst formation, all of which need to be traversed successfully in order for the embryo to develop to a new individual. These transitions are defined by exquisite remodelling of the gamete, zygote and embryo at intra- and intercellular levels, which sustains the continuation of its developmental program. Oocytes and embryos need to summon many signal transduction mechanisms, cytoskeletal 
constituents and genes in order to successfully negotiate and complete all the necessary developmental processes. Support of all these functions and processes is of cardinal importance when applying in vitro embryo production techniques to produce embryos capable of generating functional cell lines or live healthy offspring. Therefore, IVP systems are generally comprised of three stagespecific culture enviroments, specially designed to supply in the needs of each stage of oocyte, zygote and embryo development: 1) in vitro oocyte maturation (IVM), 2) in vitro fertilization (IVF), and 3) in vitro embryo culture (IVC).

Embryo production by in vitro techniques has increased steadily over the years. Among the most significant potential applications of IVP in animals are:

$>$ fundamental research on the mechanisms of early embryonic development

$>$ production of genetic merit embryos

$>$ gene injection for the transgenic animals production

$>$ cloning by nuclear transfer

Table 4.1: A comparison of the in vivo enviroment of pre-implantation embryos with in-vitro culture conditions (Rieger and Betteridge, 1993).

\begin{tabular}{||l|l||}
\hline \multicolumn{1}{|c|}{ In vivo } & \multicolumn{1}{|c|}{ In vitro } \\
\hline \hline Constant temperature & $\begin{array}{l}\text { Periods of exposure to ambient temperature } \\
\text { and varying rates of temperature change }\end{array}$ \\
\hline \hline Total darkness & $\begin{array}{l}\text { Periods of exposure to ambient light and to } \\
\text { microscope lamps }\end{array}$ \\
\hline \hline $\begin{array}{l}\text { Oxygen and carbon dioxide tensions } \\
\text { controlled by uptake and production by } \\
\text { maternal tissues }\end{array}$ & $\begin{array}{l}\text { Direct exposure to atmospheric gas } \\
\text { concentrations with little modification }\end{array}$ \\
\hline \hline Minute envelope of surrounding fluid & $\begin{array}{l}\text { Comparatively large surrounding fluid } \\
\text { volume }\end{array}$ \\
\hline $\begin{array}{l}\text { Continuous dynamic exchange of substrates } \\
\text { and metabolites between the embryo and the } \\
\text { maternal tissues }\end{array}$ & $\begin{array}{l}\text { Intermittent replacement of medium. Slowly } \\
\text { decreasing supply of substrates and dilution } \\
\text { of metabolites }\end{array}$ \\
\hline
\end{tabular}



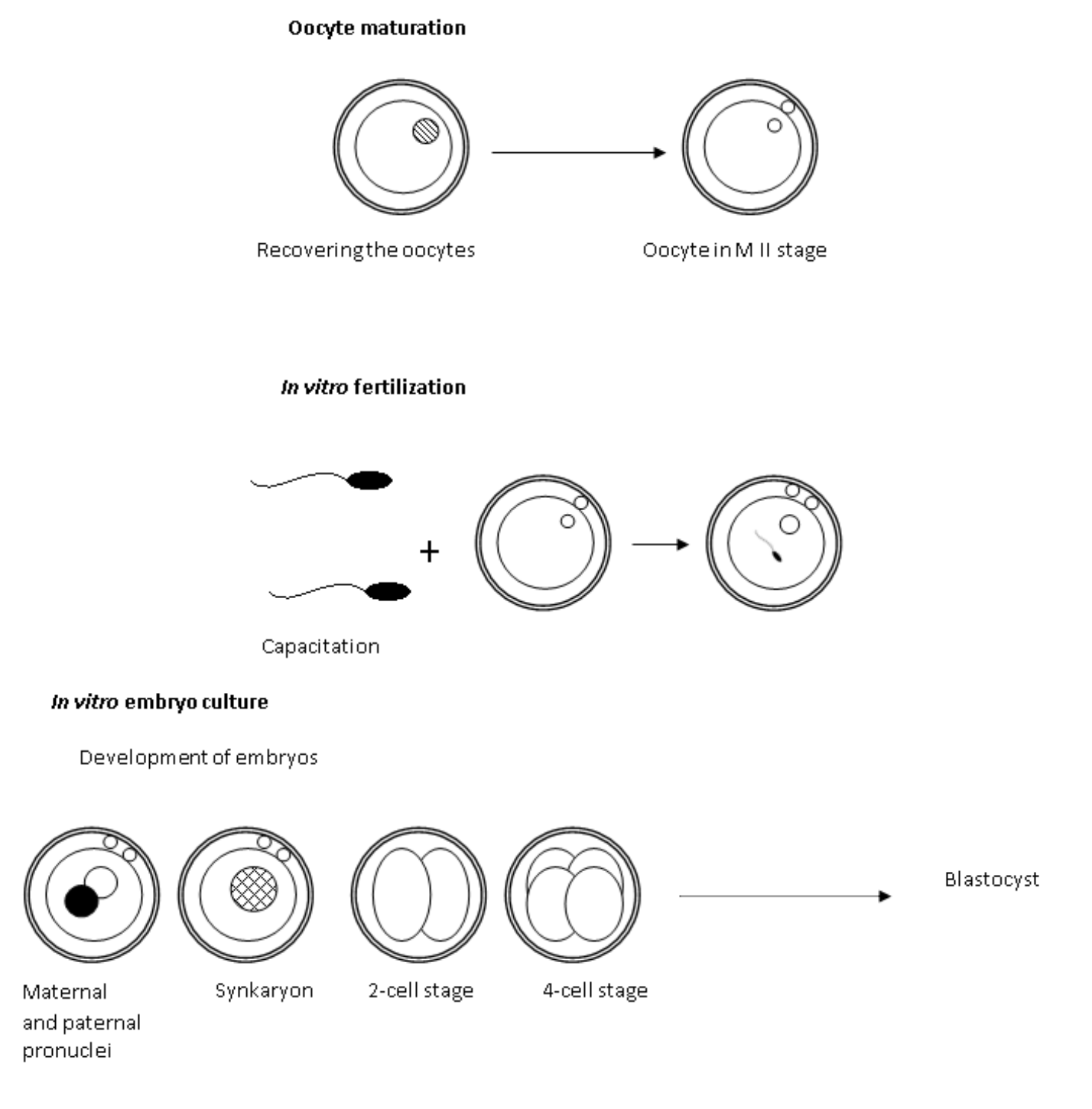

Figure 4.1: Schematic illustration of IVP.

\subsection{In vitro maturation}

\subsubsection{Recovering the oocytes}

For the set up of the techniques as well as for the large scale production of average genetic merit embryos, large quantities of material can be obtained at low cost by collecting ovaries at slaughterhouses. These ovaries are shipped to the laboratory (dry or in warmed saline) where the content of the follicles is aspirated. Cattle ovaries can be stored up to 8 hours at $25^{\circ} \mathrm{C}$ without significant loss of development competence. However, storage at $4^{\circ} \mathrm{C}$ or $37^{\circ} \mathrm{C}$ during 4 hours results in decreased oocyte viability. 


\subsubsection{Aspiration and slicing}

The needle used, as well as, the aspiration vacuum are important factors in determining the number and quality of the oocytes collected. These parameters have to be established for each species (Fig. 4.2). In the cow, good results can be obtained with 18 gauge needle connected to a $3 \mathrm{~cm} \mathrm{Hg}$ vacuum. Alternatively, the ovaries may be sliced with a razor blade (Fig. 4.3) and washed with PBS to collect the oocytes. An average of 5 to 10 oocytes can be collected from each bovine ovary (Mermillod et al. 1992) and one to two from sheep or goat ovaries during the breeding season (Cognie 1999). Prior studies have documented that follicles containing preovulatory oocytes are generally larger than those containing immature oocytes. After aspiration or slicing, the collected fluid is screened under a stereomicroscope to select the oocytes. The oocytes are surrounded by several layers of somatic cells (cumulus oophorus). These cells establish contacts together as well as with the oocyte to allow cell communication through gap junctions, which is necessary for efficient maturation of the oocyte (Van Soom et al. 2002). Only cumulus oocyte complexes (COC) with complete and compact cumulus investment should be selected (Stangl et al. 1999). Cumulus expansion observed at oocyte collection may reflect follicular atresia or premature meiotic resumption in the oocyte.

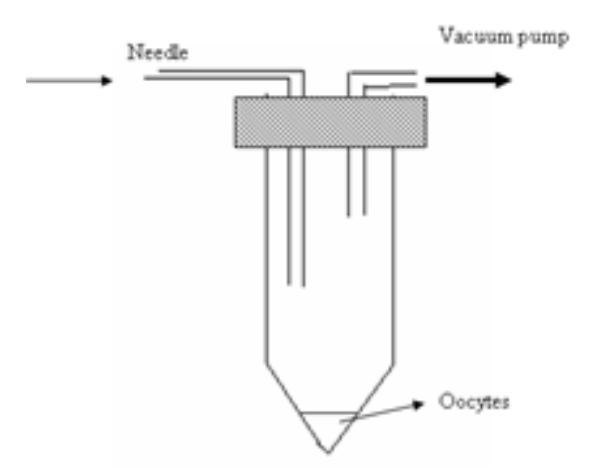

Figure 4.2: Aspiration (vacuum pump)

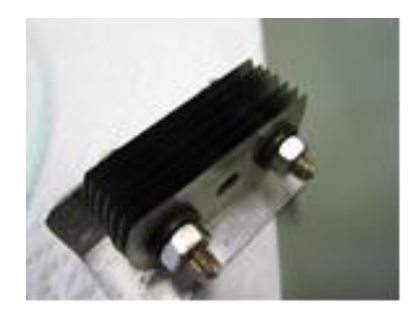

Figure 4.3: Slicing - razor blade 


\subsubsection{Ovum pick up}

An increasing proportion of in vitro produced embryos are coming from oocytes collected from live donors by ultrasound-guided follicular aspiration (Ovum Pick Up - OPU). This procedure allows the repeated production of embryos from live donors of particular value and is a serious alternative to superovulation (Fig. 4.4). Collection is performed with minimal stress to the donor and it is applied to donors all age from same month to very old animals.

In the bovine, OPU is mainly done by transvaginal aspiration of follicle content under ultrasonographic control. This technique has been developed in humans and has been then adapted in cattle (Pieterse et al. 1988). Aspiration parameters are as important in OPU procedure as they are during aspiration of oocytes from slaughterhouse ovaries. Increasing aspiration flow increases oocyte recovery rate but decreases oocyte quality (Bols et al. 1997). The ratio of oocyte collected from aspirated follicles is usually around 60-70\%. OPU could be performed as frequently as twice a week (usually Monday and Thursday), with or without hormonal stimulation of follicular growth, and allows the maximum recovery of oocytes of suitable quality for embryo production. "Once a week" collection results in the recovery of a smaller number of oocytes (of lower quality) which have already undergone cumulus expansion and atresia. This is because in the twice a week collection, if all visible follicle are aspirated no dominant follicle develops. In most once a week collection a dominant follicle is present at each successive collection that causes the regression and degeneration of the subordinate follicle. Indeed, the removal of the dominant follicles by OPU between two weekly sessions increased the blastocyst rate. Some authors prefer to combine superovulation with OPU and recover the oocytes before the onset of estrus (Garcia et al., 1998). An average of ten oocytes per cow and 8 per heifer may be collected at each OPU session in a twice a week protocol without stimulation (Galli et al. 2001). 


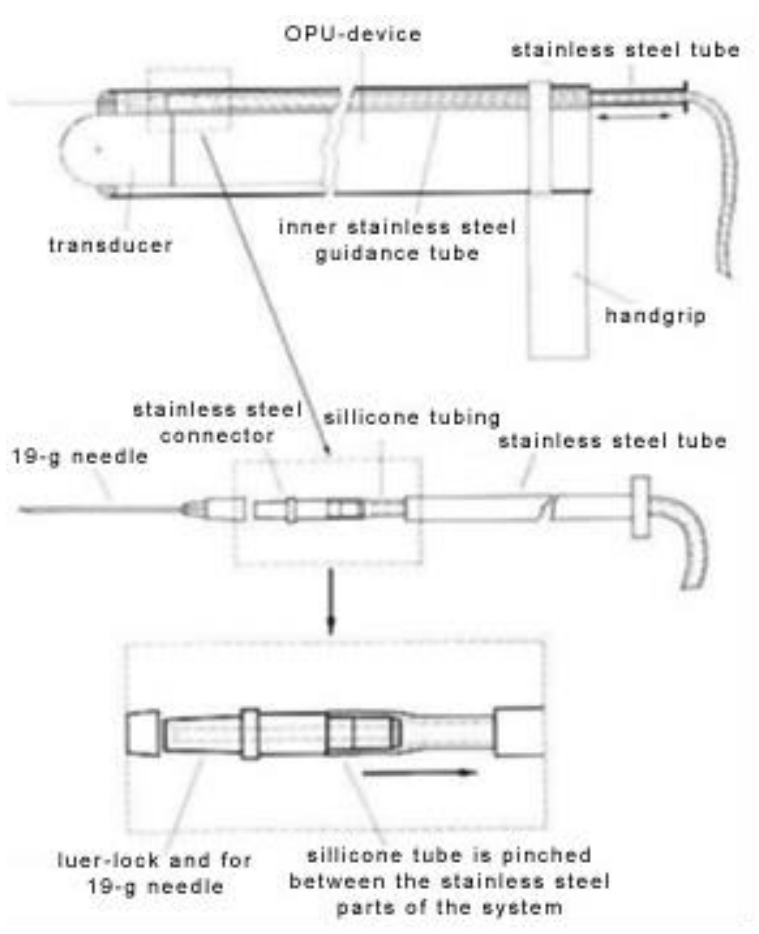

Figure 4.4: Instruments for OPU (http://www.pie-data.co.uk/Veterinary/bovappOPU.htm)

\subsubsection{Oocyte selection}

Markers for the best developmental potential of oocytes and embryos are scarce. It is believed that a mature, good quality oocyte is identified by a perfectly spherical shape, a regular zona pellucida, an intact polar body I, and a translucent, homogeneously colored cytoplasm without inclusions. It is also supposed that around the time of ovulation good quality oocytes are embedded in a wellexpanded cumulus mass and surrounded by a radiant array of corona cell layers. Although it is still controversial whether distinct oocyte morphological characteristics can predict embryo developmental potential, on the other hand, at retrieval the oocyte is difficult to assess morphologically because it is obscured by a large cumulus mass infiltrated with abundant hyaluronic acid. Therefore, in standard IVF cases in which cumulus cells are not removed, in many laboratories oocytes are inseminated in the absence of information on their morphological status.

Culture media have been adapted to suit the specific individual needs of the oocyte and embryo (Petters and Wells, 1993), not much progress in the improvement of embryo viability has been made since the adoption of its use a decade ago. Chronic and cumulative stresses caused by sub-optimal culture conditions or insufficiencies in the constituents of the culture medium, can lead to oocyte or embryo anomalies at a genetic or metabolic level and severely jeopardise the viability of the resultant embryo (Abeydeera, 2002). 


\subsubsection{Oocyte maturation}

Oocyte maturation is a complex phenomenon during which the oocyte progresses from the diplotene phase to the metaphase II stage. This process includes nuclear maturation (reflects the modification of the chromatin status from the dictyate phase to the Metaphase II stage) and cytoplasmic maturation (encompasses all the changes in distribution and organization of the individual organelles from the GV to the Metaphase II stage).

All primary oocytes in ovary are blocked at the prophase stage of the meiotic cycle. The block of meiotic progression during follicular growth is controlled by factors produced by the surrounding somatic cells. During early foliculogenesis, the oocyte grows, undergoes strong morphological modifications and stores molecules (proteins, RNAs) that will be determinant for the success of fertilization and early embryo development.

\subsubsection{Nuclear maturation}

The transition from the diplotene stage to metaphase is called diakinesis and is the basis of in vitro maturation. During diakinesis, the nuclear membrane starts to fold, the nuclear pores disappear and then the nuclear membrane fragments before rapidly disappearing to leave only small sacs with double walls (Kubelka et al., 1988). These events are known as germinal vesicle breakdown (GVBD), which is the first visible sign of meiotic resumption. The nucleolus disappears rapidly after coming in contact with the cytoplasm. In cattle, GVBD occurs within hours after removal from the follicle or the ovulatory LH signal. Then, the chromosomes condense further. The kinetochores appear and the microtubules pull the chromosomes and they form the metaphasic plate of MI. The separation of the homologous chromosomes and the migration of the chromosomes to their respective poles take place during anaphase I. During telophase I, the chromosomes found at each pole are surrounded by a nuclear membrane. The second meiotic division without chromosome replication takes place immediately and the oocyte reaches the metaphase II. The oocyte remains arrested at the metaphase II stage until fertilization takes place and the oocyte completes meiosis and forms the pronucleus.

\subsubsection{Cytoplasmic maturation.}

The cytoplasmic maturation involves transformations at the cytoplasmic level that prepare the cell to support fertilization and early embryonic development. For example cytoplasmic maturation includes mitochondria repositioning and the migration of cortical granules in the cortical region of the ooplasma. The completion of nuclear maturation alone does not guarantee subsequent embryo development (Yang et al., 1998). 


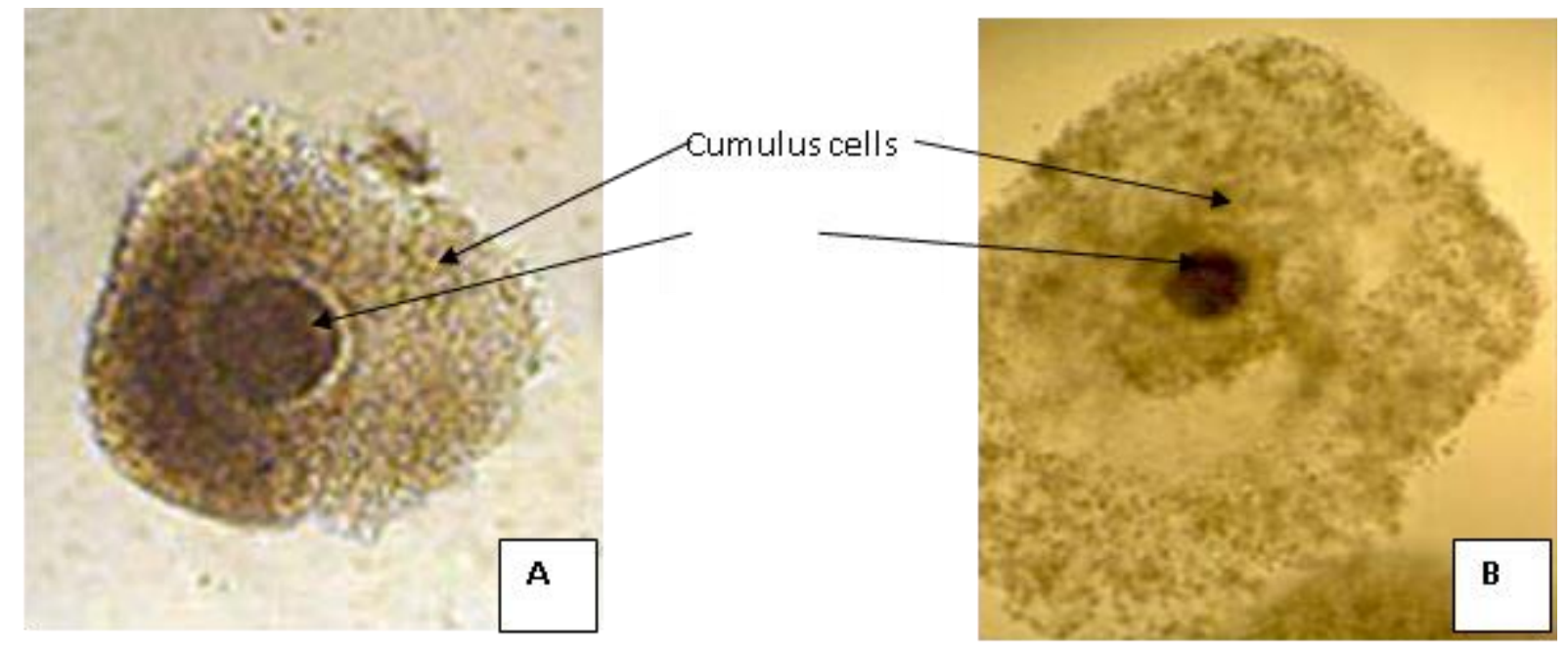

Figure 4.5: Bovine cumulus-oocyte complex (COC) with compact layers of cumulus cells at the beginning of in vitro maturation (A) and after maturation $(B)$.

\subsubsection{Maturation medium}

In vitro maturation is probably the most critical part of the whole process of in vitro embryo production. Ruminant oocytes are usually matured at $39^{\circ} \mathrm{C}$ under a $5 \% \mathrm{CO} 2$ in a humidified atmosphere. The optimal maturation time (more than $90 \%$ of the oocytes at the metaphase II stage) is $22-24 \mathrm{~h}$ but by porcine oocytes this period takes until $44-48 \mathrm{~h}$. Due to the high lipid content of oocytes cytoplasm, it is not possible to follow the progression of their nuclear status during culture. Additionally, the presence of the cumulus cells is required during IVM for efficient cytoplasmic maturation and these cells mask the oocyte. Consequently, the only visible sign of oocyte maturation during IVM is the expansion of the cumulus cells. These cells produce hyaluronic acid which is secreted and polymerized in the extracellular matrix, leading to the increase of intercellular space.

Several culture media have been proposed for IVM (MEM, Waymouth, Ham-F12). The most common media used in culture system are TCM199 medium, bicarbonate buffered, NCSU 37 and containing minerals, carbon and energy sources (glucose, glutamine) as well as vitamins and amino acids. The medium is generally supplemented with high molecular weight molecules that exert a surfactant effect (bovine serum albumin - BSA) and provide hormones and growth factors (fetal calf serum, serum of female in estrus, follicular fluid). These complex additives from animal sources may raise sanitary questions due to possible presence of pathogen agents, and decrease the reproducibility 
of experiments. They may be successfully replaced by high molecular weight polymers (Ali and Sirard, 2002) for the surfactant effect (such as polyvinyl alcohol) and by cocktails of purified or recombinant hormones and growth factors (Lonergan et al. 1996).

Glucose is the main energetic substrate consumed by cells; however, it elicits harmful effects on the early development of pre-implantation mammalian embryos (Bavister, 1995). Pyruvate and lactate are the preferential energetic substrates consumed by the early embryo during in vitro development (Pinyopummintr and Bavister, 1996). At early cleavage stages, glucose might impair embryonic development, inhibiting oxidative phosphorylation through glycolytic metabolites (Bavister, 1995) when embryos depend mainly on this route for generating energy (Thompson, 2000). Increased glucose concentration may also cause deviation in embryo male to female ratio (Bredbacka and Bredbacka, 1996) by retarding the development of female embryos.

Albumin is one the most prevalent proteins of the mammalian reproductive tract and it may have a nutritive role during embryo development post-compaction. BSA is still a biologic component subject to contamination that may impair embryo and fetal development, and its role in in vitro culture is not very clear (Thompson, 2000). A possible role of BSA in embryonic development may be to provide amino acid substrates for embryo metabolism, which could favor embryo development.

Hormones are usually added to the maturation medium (FSH, LH, estradiol), in addition to biological fluids. Their exact function is not clearly established, although the effect of FSH on cumulus expansion and metabolism has been clearly established (Sutton-McDowall et al. 2004). Estradiol effecting oocyte is exerted through intracellular receptors and seem to be detrimental to meiotic progression, inducing chromosomal abnormalities (Beker-van Woudenberg et al. 2004). Growth hormone $(\mathrm{GH})$ stimulates some aspects of cytoplasmic maturation (cortical granules migration) as well as nuclear maturation, resulting in a higher blastocyst yield (Bevers and Izadyar, 2002). GH action seems to be mediated by cumulus cells (Izadyar et al. 1997). Epidermal growth factor (EGF) has been shown to stimulate nuclear and cytoplasmic maturation in a wide variety of species including mouse, pig, cattle, deer goat and sheep (Mermillod et al., 2006).

The effect of oxygen tension on the development of in vitro fertilized embryos seems to be dependent on the culture media or culture systems used. Low oxygen tension in embryo culture may contribute to reducing free-radical formation. Free radicals impair embryo metabolism and development (Lane, 2001). High oxygen tension may cause apoptosis in embryonic cells (Yuan et al., 2003) and/or alter the embryonic gene expression pattern (Harvey et al., 2004).

Another important aspect of COC environment during IVM is the antioxydative potential of the IVM medium (Ali et al. 2003). As all living cells, oocyte is sensitive to reactive oxygen species (ROS) that could induce detrimental chemical reactions (lipid or protein peroxydation). To overcome 
possible ROS detrimental effects during culture, antioxydative molecules (mercaptoethanol, cystein, vitamin A C, E), divalent cations chelators (EDTA, taurine, hypotaurine, transferrine) or ROS scavengers (superoxyde dismutase, catalase) could be added to maturation medium (Kobayashi et al. 2006).

\subsection{In vitro fertilization}

The overall success of the in vitro fertilization process requires good control of different steps: sperm selection, sperm capacitation and fertilization itself. Semen is known to contain factors that can prevent capacitation and/or fertilization. Abnormal spermatozoa, dead cells, enzymes and bacteria are also present that could be the cause of IVF failure or unreproducible results. In addition, the concentration of sperm suspensions has to be controlled to ensure the continuity of the IVF method. Numerous techniques have been proposed for removing undesirable semen components and concentrating the motile sperm fraction in a suspension of known concentration. The most conventional by cattle is the swim-up method but other methods have been described involving either centrifugation on BSA or Percoll density gradients or Sephadex column separation (Mermillod et al., 1992). In the swim-up method, spermatozoa are deposit at the bottom of a culture medium containing tube and allowed to swim in the medium. The top fraction, containing motile sperms is then collected after a given incubation time (Fig. 4.6). Alternatively, motile sperms may be purified by centrifugation in a discontinuous density gradient (Percoll). The motile fraction is collected at the bottom of the tube after centrifugation.

The next critical point encountered in IVF is sperm capacitation. Ejaculated spermatozoa are more suitable than epididyme collected sperm for bovine IVF and give rise to more normal eggs but they have to undergo a capacitation treatment prior to fertilization (First and Parrish, 1987). The mechanisms of mammalian sperm capacitation, which normally takes place in the female genital tract are not yet well understood but several in vitro capacitation methods have been proposed: washing with high ionic strength solution (sperm need a high intracellular $\mathrm{K}+$ concentration and low $\mathrm{Na}+$ intracellular concentration); treatment with follicular fluid or oviduct fluid: co-culture onto oviduct cell mono layers; incubation with the Ca2+ ionophore A23187. However, the most common method used in bovine IVF involves heparin. Heparin, as well as other glycosaminoglycans present in the female genital tract, is able to capacitate bovine fresh sperm within $4 \mathrm{~h}$ and frozen-thawed sperm within 15 min (Parrish et al., 1986). Caffeine has also been added to insemination medium.

The sperm concentration used for IVF ranges between 0.5 and $2 \times 106$ spermatozoa per $\mathrm{ml}$ in fertilization medium. This concentration should be optimized for each male and even for each ejaculate from a given male. 
Apart from different environment one very striking difference between in vivo and in vitro fertilization is the oocyte-spermatozoon ratio and the presence or absence of cumulus oophorus. In vivo the ratio is close 1:1 (Hunter, 1996); in vitro it si approximately 1:104; in vitro the cumulus oophorus is not shed spontaneously from the maturated oocytes; in vivo it is shed a few hors after ovulation.
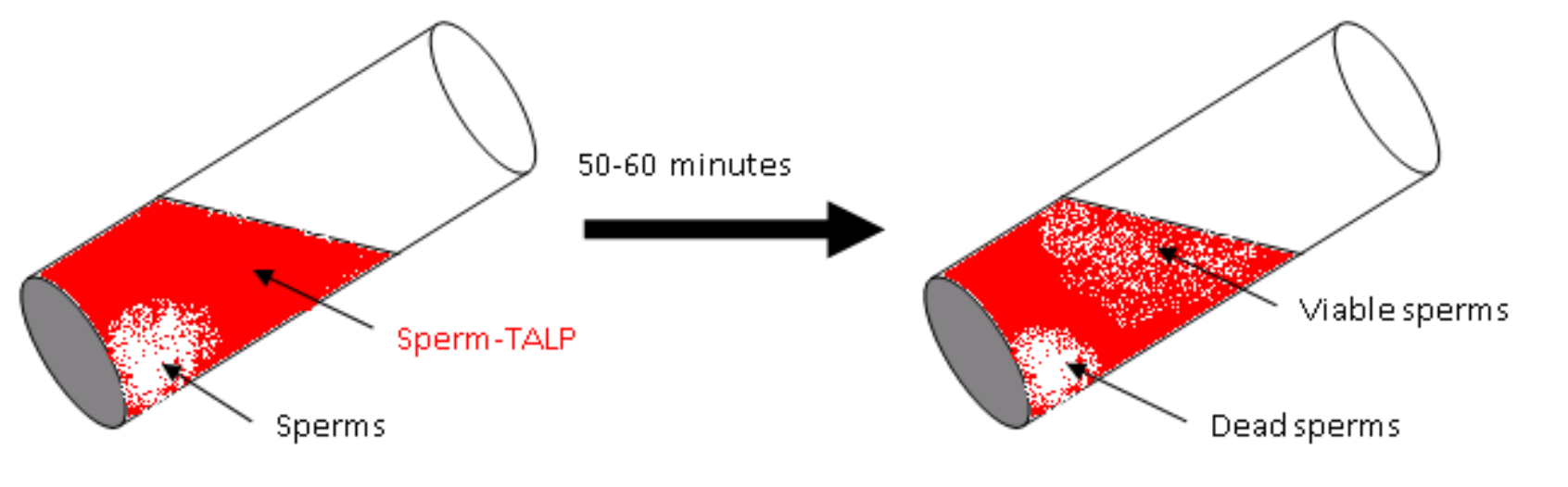

Figure 4.6: Swim-up method

A number of approaches employing micro-manipulation of the eggs and sperm have been advocated. These include zona cutting and zona drilling in which a hole is cut in the zona pellucida (the shell) of the egg to allow the sperm to more easily enter the egg. . The sperm can then hopefully fuse with the cytoplasm and fertilize the egg. The sperm must have some reasonable progressive motility for this to occur. These methods did not work particularly well and gave an unacceptably high level of abnormal fertilization of the eggs.

\subsection{In vitro cultivation}

In standard cell culture conditions, ruminant embryo development may be blocked at the 8-16 cell stage. This block was first overcome through the use of co-culture systems involving oviduct epithelial cells (Eyestone et al. 1991) or other cell lineage (Myers et al. 1994) in standard tissue culture media (TCM199). The effect of cells has not been clearly established. They may produce some growth factors positively regulating embryo development or remove toxic media components (Killian 2004). In a second step, new culture media have been designed to more exactly fit embryo requirements without the need for co-culture. One of the first media designed for this purpose was synthetic oviduct fluid or SOF (Fukui et al. 1991). The composition of SOF was established from the 
composition of bovine oviduct fluid. However, SOF or SOF modifications have also been successfully used in small ruminant species. More recently, sequential media have been proposed to fit the evolution of embryo requirements during early development (Gardner et al. 2000). For example, glucose seems to be toxic to early cleaving embryos, whereas morula needs some glucose in the medium for further development (Donnay et al. 2002; Gardner et al. 2000).

Semi defined media represent an easy way to produce mammalian embryos in vitro. However, embryo quality (viability after cryopreservation and/or transfer to recipient) appears to be low after development in such systems (Garcia-Garcia et al. 2006). On the other hand, co-culture systems may be more complicated to manage but allow the production of higher quality embryos. Therefore, some commercial bovine IVP teams still use co-culture system or embryo development in the oviduct of transient recipient ewes. In this case, the embryos are transferred in the oviduct of synchronized ewes just after IVF and surgically collected seven days later (Galli et al. 2004). This IVP system allows the production of embryos with similar quality and cryotolerance as in vivo collected ones.

The development usually takes place at $39^{\circ} \mathrm{C}$ in droplets of culture medium overlaid with oil for 7 to 9 days (Fig. 4.8). A high density of embryos per volume unit (one embryo per $1 \mu$ l) seems beneficial to development (Ferry et al. 1994). When embryos are cultured without cell support, the oxygen concentration in atmosphere should be reduced to $5 \%$ to avoid induction of oxidative stress on the embryos (Olson and Seidel, 2000). As during IVM, addition of antioxidant molecules may increase rate or quality of IVP embryos (Dalvit et al. 2005). Serum is usually added to the culture medium from the beginning or after the first cleavages (48h post insemination). The presence of serum accelerates the development of embryos but seems to negative effects on their quality (Rizos et al. 2003) translating into fetal and placental abnormalities (Large Offspring Syndrome, see below) reported during pregnancy (Thompson et al. 1995). The rate of cleavage is usually high (over $80 \%$ of the total oocytes), when appropriate bull and fertilization conditions are used. However, the rate of development to the blastocyst stage plateaus at around $40 \%$ of the total oocytes. It has been clearly established that oocyte quality (intrinsic quality of primary oocyte coupled to maturation conditions used) determines the rate of blastocyst production, whereas development environment is determinant for embryo viability (Rizos et al. 2002). 


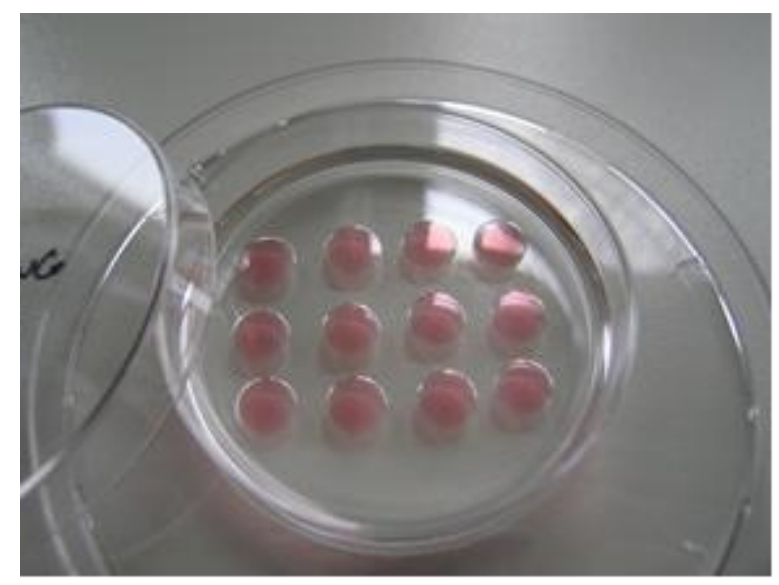

Figure 4.8: Droplets of culture medium overlaid with oil.

The pregnancy rates obtained after transfer of fresh IVP embryos are usually good (40 to 50\% of calves born). Some problems of calf abnormality and perinatal mortality have been reported (Farin et al. 2006). However, these problems are less frequent than those observed with cloned embryos and seem to be linked to in vitro environment and to the presence of serum in the culture medium that induces chromosomes aberrations, modifications of embryo gene expression pattern and metabolism (Rizos et al. 2003).

The main limitation to a wider use of OPU - IVP technique in breeding schemes is the low cryoresistance of the resulting embryos. Although well established techniques of embryo freezing are described in cattle, sheep and goat and provide good results of pregnancy rate after transfer of the thawed embryos, these techniques appeared sub-optimal for the cryopreservation of IVP embryos. Several alternative methods of embryo cryopreservation have been proposed to overcome the low viability of these embryos. Some vitrification procedures (high speed cooling with high concentration of cryoprotectants leading to amorphous solidification without ice crystals formation) appeared to partially solve the problem. However, the quality of the embryo which is determined by the culture conditions, appears to be one of the critical factors. Similar pregnancy rates have been observed after $10 \%$ glycerol slow freezing for in vitro fertilized embryos grown in sheep oviduct than for in vivo derived embryos (Galli et al. 2004). This indicates that culture conditions still remain to be improved to better mimic the in vivo conditions.

\subsubsection{Characteristic of early embryo stage}

The product of fertilization is a one-cell embryo with a diploid complement of chromosomes. Over the next few days, the mammalian embryo undergoes a series of cell divisions, ultimately 
leading to formation of a hollow sphere of cells known as a blastocyst. At some point between fertilization and blastocyst formation, the embryo moves out of the oviduct, into the lumen of the uterus.

All of the early stages, the embryo is encased in its zona pellucida. Embryos from other mammals have a very similar appearance, and the general sequence of stages is seen in all mammals.

Unfertilized oocytes typically fill the entire space inside the zona pellucida, but after fertilization, the one-cell embryo usually is somewhat retracted from the zona pellucida surrounding it. Although not visible in this image, one or two polar bodies are often visible in the perivitelline space, the area between the embryo and the zona pellucida.

The one cell embryo undergoes a series of cleavage divisions, progressing through 2-cell, 4-cell, 8-cell and 16 cell stages. The cells in cleavage stage embryos are known as blastomeres.

Early on, cleavage divisions occur quite synchronously. In other words, both blastomeres in a two-cell undergo mitosis and cytokinesis almost simultaneously. For this reason, recovered embryos are most commonly observed at the two, four or, and seen here, eight-cell stage. Embryos with an odd number of cells (e.g. 3, 5, 7) are less commonly observed, simply because those states last for a relatively short time.

Soon after development of the 8-cell or 16-cell embryo (depending on the species), the blastomeres begin to form tight junctions with one another, leading to deformation of their round shape and formation of a mulberry-shaped mass of cells called a morula. This change in shape of the embryo is called compaction. It is difficult to count the cells in a morula.

Formation of junctional complexes between blastomeres gives the embryo and outside and an inside. The outer cells of the embryo also begin to express a variety of membrane transport molecules, including sodium pumps. One result of these changes is an accumulation of fluid inside the embryo, which signals formation of the blastocyst.

As the blastocyst continues to accumulate blastocoelic fluid, it expands to form - it an expanded blastocyst. The blastocyst stage is also a landmark in that this is the first time that two distinctive tissues are present. A blastocyst is composed of a hollow sphere of trophoblast cells, inside of which is a small cluster of cells called the inner cell mass. Trophoblast goes on to contribute to fetal membrane systems, while the inner cell mass is destined largely to become the embryo and fetus. In the expanded blastocyst shown here, the inner cell mass is the dense-looking area at the bottom of the embryo.

Eventually, the stretched zona pellucida develops a crack and the blastocyst escapes by a process called hatching. This leaves an empty zona pellucida and a zona-free or hatched blastocyst lying in 
the lumen of the uterus. Depending on the species, the blastocyst then undergoes implantation or elongates rapidly to fill the uterine lumen.

The length of time required for preimplantation development varies somewhat, but not drastically, among species.

In addition to the morphological changes in the embryo described here, preimplantation development is associated with that might be called an awakening of the embryonic genome. There is, for instance, little transcription in the embryos of most species prior to the 8 cell stage, but as embryos develops into morulae, then blastocysts, a large number of genes become transcriptionally active and the total level of transcription increases dramatically.
Abbreviations:
BSA - bovine serum albumin
COC - cumulus oocyte complexes

EDTA - ethylenediaminetetraacetic acid

EGF - Epidermal growth factor

FSH - follicle stimulating hormone

GH - Growth hormone

GV - germinal vesicle

GVBD - germinal vesicle breakdown

ICSI - Intra-Cystoplasmic Injection

IVC - in vitro oocyte/embryo cultivation

IVF - in vitro oocyte fertilization

IVM - in vitro oocyte maturation

IVP - in vitro embryo production

LH - luteinizing hormone

MI - metaphase I

MII - metaphase II

OPU - Ovum Pick Up

PBS - Phosphate-buffered saline

ROS - reactive oxygen species

SUZI - Sub-Zonal Sperm Injection 


\section{Cryopreservation of oocytes and embryos}

\subsection{Introduction}

The purpose of cryopreservation is to slow down or virtually stop the biological time for living creatures including mammalian gametes and early embryos. Analogues of the procedure have been used by the Nature itself for the survival of some primitive organisms including viruses and bacteria under unfavorable conditions, i.e. trapped by ice, or hibernation of animals, even some mammals during the cold season. Recent advancement in cryopreservation - i.e. deep cooling to temperatures where all chemical processes slow down to an undetectable level - means, however, a situation that under physiological conditions cells or organisms aren't expected to meet and most of them would die without our assistance.

\subsection{The effect of temperature}

Curiously, it is not the extremely low temperature that causes the problem. Storage at $-150^{\circ} \mathrm{C}$ or below (a temperature that is most commonly, almost exclusively achieved by submerging samples into liquid nitrogen) is almost entirely harmless, and can last for hundreds, maybe thousands of years. Former concerns regarding the potential harmful effect of the background (cosmic) radiation seem to be unjustified. Although on these low temperatures the usual DNA repair mechanisms cannot function, the damage may be much less extensive than supposed and does not significantly influence viability (Glenister and Lyon, 1986).

The real problem is the road to and from the storage temperature, i.e. the cooling and the warming procedure. Various structures suffer various damages during the passage through the different temperature zones. The first type of injuries, chilling damage occurs at relatively high temperatures, between +15 (or even +20 ) and $-5^{\circ} \mathrm{C}$. The most chilling-sensitive structures of mammalian cells are lipid droplets, lipid-rich membranes and microtubules (Martino et al., 1996a). The damage of microtubules may be reversible; however, their temporary malfunction may have irreversible consequences for further development, for example by causing chromosome abnormalities. Damage of lipids, especially lipid droplets, on the other hand, seems to be irreversible and may lead to the progressive disintegration of the cellular structure and function. Actually there are very few ways to avoid chilling injury: one possibility is to remove the sensitive structures from the cytoplasm (for example separation of lipid droplets from the rest of the cytoplasm, see discussed later), or application of extremely high cooling and warming rates to decrease radically the time cells spend in this temperature zone. 
The next temperature zone, between -5 and $-80^{\circ} \mathrm{C}$ results in the most commonly known type of damage, ice crystal formation (Mazur, 1970). Ice crystals may destroy all organelles and membranes predominantly by a simple mechanical force that occurs during their formation and growth. The most common approach to decrease or eliminate this type of injury is the application of cryoprotectants, substances that prevent or minimize ice crystal formation. The chemical structure and the biological effect of these cryoprotectants is rather diverse (see discussed in detail later), however, apart from the beneficial main effect for ice crystal formation, all of them are more or less toxic, and they addition also causes osmotic stress. The other widely used approach to control ice crystal formation is the application of strictly determined rates for cooling and warming.

The final type of damage may occur at approximately between -50 to $-150^{\circ} \mathrm{C}$ and consists of mechanical fractures, crude destructive damages of organisms (Kasai et al., 1996). Unequal growth of ice crystals and subsequent fracture between two solid masses is supposed to be responsible for most of these injuries, although the damage may also occur in systems where ice crystal formation is completely prevented. The scissor-like effect of two solid masses slipping on each other is well visible on the sharp lines of fractures. Predisposing factors to this type of injury are high cooling and warming rates and closed containers, i.e. the commonly used sealed plastic straws filled both with gas and solution columns. Under such circumstances the extreme pressure changes caused by the shrinking or expanding air during cooling and warming, respectively, may induce breakage in the solidified liquid and in the (relatively frequent) unfortunate cases these breakages result in fracture of the biological material, as well.

The described types are just the major known form of cryoinjuries induced partially by the low temperatures (chilling, ice formation, fracture), or by our interventions to minimize the harmful effect (osmotic and toxic effect of cryoprotectants). Unfortunately, due to the special situation, the delicate molecular changes that happen on a given temperature are very difficult to identify, and a retrospective analysis of the events may become completely misleading. Accordingly, most cryopreservation techniques (especially those used in reproductive biology) have been established and fine-tuned empirically, sometimes just by chance, and the underlying mechanisms have been clarified later (if and when our actual knowledge permitted such a clarification).

\subsection{Other factors influencing cryoinjuries}

Apart from the temperature, there are many other factors that influence the seriousness of the damage. A general rule (with many exceptions) is that the smaller the sample is, the higher its chances are to survive cryopreservation. Viruses and bacteria may survive deep freezing without any artificial support, and sometimes against our best intentions, making storage tanks permanently infected and 
liquid nitrogen mediated disease transfer possible (Bielanski et al., 2003). Several types of mammalian cells cultured on monolayers (fibroblasts, epithelial cells. etc.) can be cryopreserved by using a simple procedure and by using a single cryoprotectant solution. In most mammalian species, the smaller spermatozoa survive better cryopreservation than embryos consisting of larger cells; and blastocysts consisting of 30 to 150 cells and a large cavity filled with solution are less sensitive than oocytes that have approximately the same size, but are composed from a single cell.

However, size is not the only determining factor. Human zygotes, for example are easier to cryopreserve than human unfertilized oocytes, and in most mammalian species, there is a considerable difference between the sensitivity of MII phase oocytes and those in the germinal vesicle stage (the former ones survive better the procedure) in spite of the similar size and shape. The reason of these differences (with a commonly used but rather euphemistic expression) is only partially understood.

The almost completely spherical shape of oocytes and zygotes may mean an addtional problem. For the appropriate effect, some cryoprotectants should be equally dissolved in each part of the cell. However, to reach this equilibrium may take a considerable amount of time and the toxic effect during this prolonged exposure may kill cells before any cooling is started. Accordingly, most cryopreservation methods try to find a fragile balance, to keep overexposition of the periphery still tolerable while providing appropriate protection to the central part, as well.

The problem described above also highlights another factor: permeability. It may differ considerably between developmental stages and species, and may require the selection of the correct equilibration and dilution parameters (the way cryoprotectants are added to and removed from the cells, including concentrations and temperatures) as well as application of cryoprotectants that are most appropriate for the given purpose.

Apart from the size, shape and permeability, supposedly a number of other, poorly understood or entirely unknown factors may also determine cryosurvival. Their effect differs from species to species, may change between developmental stages, and is influenced also by the origin or embryo quality: in vitro produced embryos are generally regarded handicapped compared to their in vivo counterparts, accordingly their survival rates after cryopreservation are lower, either.

\subsection{Cryopreservation in mammalian embryology}

Initial successes in reproductive cryobiology of mammals were achieved in the middle of the last century, first in spermatozoa, later in embryos and oocytes (Table 1). Techniques applied for domestic and experimental animals were rapidly adopted also in human embryology, with profound, sometimes controversial moral and philosophic consequences. Although few restrictions are applied when gametes are in concern, cryopreservation of human embryos is unlawful in many countries. To lessen 
the serious consequences for human artificial reproduction, zygotes (with two separate pronuclei for male and female chromosomes) are not considered as one-cell embryos but as pronuclear stage oocytes. (The rather paradox category of "pre-embryo" for all used by human reproductive biologist for all preimplantation stages may also be explained as a -rather desperate - attempt to escape legal restrictions.)

Table 5.1: Significant achievements and important techniques in cryopreservation of mammalian oocytes and embryos. Note: achievements without further confirmation by using the same procedure marked with (?)

\begin{tabular}{|l|l|}
\hline \hline Polge, 1952: & freezing of bull spermatozoa \\
\hline \hline Whittingham et al., & freezing of mouse embryos \\
\hline \hline Wilmut et al., 1973: & freezing of bovine embryos \\
\hline Willadsen, 1977: & efficient freezing of embryos, ultimate slow freezing technique \\
\hline \hline Whittingham, 1977: & freezing of mouse oocytes \\
\hline \hline Trounson et al., 1983: & freezing of human embryos \\
\hline Rall and Fahy, 1985: & vitrification of mouse embryos \\
\hline Massip et al., 1986: & vitrification of bovine embryos \\
\hline Nakagata, 1989: & vitrification of mouse oocytes \\
\hline \hline Fuku et al., 1992: & freezing of bovine oocytes \\
\hline \hline Chen, 1986: & freezing of human oocytes \\
\hline Hamano et al., 1992: & vitrification of bovine oocytes \\
\hline Vajta et al, 1998a & efficient vitrification of animal oocytes and embryos, OPS \\
\hline \hline Kuleshova et al., 1999: & vitrification of human oocytes (?) \\
\hline $\begin{array}{l}\text { Kuwayama et al., } \\
\text { 2005a: }\end{array}$ & efficient vitrification of human oocytes and embryos, Cryotop \\
\hline \hline
\end{tabular}

In spite of our limited knowledge, it can be certainly stated that all mammalian cells and organisms including oocytes and embryos suffer a considerable damage during the cryopreservation procedure. However, they also have a considerable ability for regeneration to survive the event without definite loss in their developmental competence. The purpose of all cryopreservation procedures is to minimize the injuries and to facilitate the regeneration.

As ice crystal formation is the major source of cryoinjuries, basically all cryopreservation strategies focus on this problem, while also attempting to control other harmful mechanisms. As described earlier, there are two major ways to prevent ice formation: addition of cryoprotectants and determine cooling and warming rates. 
The common feature of cryoprotectants is the ability to decrease cryoinjuries. A wide range of materials fits this definition, including simple, defined, low molecular weight solvents as ethanol; or complex, partially undefined biological compounds like sera or egg yolk. They may either enter the cell (permeable cryoprotectants; for example ethylene glycol, propylene glycol, dimethylsulphoxide, glycerol) or remain outside (non-permeable cryoprotectants including sucrose, trehalose, polyethylene glycol, etc.). The main supposed effect of permeable cryoprotectants is to minimize ice formation. A similar mechanism, the osmotic dehydration may play a considerable role in the protective effect of non-permeable cryoprotectants. However, both permeable and non-permeable cryoprotectants may also have additional protective mechanisms, for example to stabilize intracellular structures and the cell membrane. Unfortunately, most cryoprotectants have some negative effect, including toxic and osmotic injuries. Toxicity is usually proportional to the concentration of the substance and to the time of exposure (at physiological temperatures).

Cooling rates may vary according to the applied method. It may start with a moderate or stepwise cooling between the physiological temperature to approx. $-6^{\circ} \mathrm{C}$, then very slow and highly controlled cooling rates to -30 or even $-80^{\circ} \mathrm{C}$, followed by a very rapid phase achieved by plunging the sample to the $-196^{\circ} \mathrm{C}$ liquid nitrogen. In other methods the whole cooling throughout the entire temperature range is either rapid (around $200^{\circ} \mathrm{C} / \mathrm{min}$ ) or ultrarapid (up to $20.000-100.000^{\circ} \mathrm{C} / \mathrm{min}$ ). Warming may be also performed stepwise, with highly controlled or just slightly delayed increase of the temperature, or (more typically) rapidly, including the commonly achievable highest rates of temperature change described above. Cryoprotectant composition, addition, concentration and removal, as well as warming rates are more or less determined by the selected cooling rates.

\subsection{Main cryopreservation strategies}

During the past decades, two major groups of methods have been developed: the more traditional slow freezing and vitrification. Storage, warming and rehydration, i.e. removal of cryoprotectants differ only slightly between the two procedures (with some exceptions), the main difference exists in the addition of cryoprotectants and cooling.

Slow freezing is an attempt to create a delicate balance between various damaging factors including ice crystal formation, fracture, toxic and osmotic damage. Embryos and oocytes are typically equilibrated in 1 to $2 \mathrm{M}$ solutions of permeable and non-permeable cryoprotectants, loaded into $0.25 \mathrm{ml}$ straws, sealed and cooled relatively rapidly to $-7^{\circ} \mathrm{C}$ by placing the straws into the (sophisticated and rather expensive) controlled-rate freezer. At $-7^{\circ} \mathrm{C}$, seeding, i.e. ice crystal formation is induced in the solution preferably far from the embryo or oocyte by touching the straw with a cool forceps. The subsequent steps are entirely or almost entirely performed by the controlled- 
rate freezer. Subsequent, highly controlled cooling rates are between 0.3 and $1^{\circ} \mathrm{C} / \mathrm{min}$. The controlled rate cooling then usually continues to around -30 or $-40^{\circ} \mathrm{C}$. When straws reach these temperatures, they are immersed to liquid nitrogen for final cooling and storage.

In slow freezing, the toxic and osmotic damage caused by the relatively low concentration of cryoprotectant solutions may not be too serious. However, this concentration is insufficient to eliminate ice crystal formation; therefore an additional manipulation is required to minimize the damage. It is the slow cooling and seeding that results in controlled growth of ice in the extracellular solution, consequently a considerable increase of the concentration of ions, macromolecules and other components - including cryoprotectants - occurs in the remaining fluid containing the sample. The slow rate of the procedure allows solution exchange between the extracellular and intracellular fluids without serious osmotic effects and deformation of the cells. Although the concentration of these components - especially of cryoprotectants - seems to be dangerously high at the final phases, it happens at low temperatures, (below $-20^{\circ} \mathrm{C}$ ) where the real toxic effect is minimal. On the other hand, this elevated concentration minimizes ice crystal formation allowing solidification of most of the intracellular water in a glass-like form.

This phenomenon fulfills the physical criteria of vitrification. However, in cryobiology this definition is mainly reserved for the other major group of cryopreservation methods, where the whole sample including both the extracellular and intracellular solutions vitrifies. The name refers the macroscopic appearance, where at vitrification the solution should remain entirely clear and transparent, glass-like or vitrous. One may also regard this type of solidification as an extreme increase of the viscosity of the solution. However, the frequently emphasized opinion that vitrification requires increased viscosity of solutions that is achieved by viscous cryoprotectants is a pure misinterpretation of facts.

Vitrification, as a method to cryopreserve biolgical samples may be regarded as a more radical approach than slow freezing, as one of the main sources of injuries, ice crystal formation is entirely eliminated. However, a negative consequence of this strategy is the increased probability of other forms of injury especially those related to the cryoprotectants.

Vitrification of solutions requires a radical increase of both the cooling rates and the concentration of cryoprotectants. The higher the cooling rate, the lower the required cryoprotectant concentration is, and vice versa. Theoretically, with the extreme increase of cooling rate (to approx. $107^{\circ} \mathrm{C} / \mathrm{s}$ ) vitrification could also be achieved in pure water, but the usual limits, especially in embryology are far below this rate (Rall, 1987). In practice, vitrification requires two- or even fourfold increase of cryoprotectant concentration compared to traditional freezing. The art of a successful vitrification method is 1 . to establish a safe system for maximal and reliable cooling (and warming) 
rates while avoiding consequent damages including fracture of the zona pellucida or the cells, and 2. to eliminate or minimize toxic and osmotic effects of high cryoprotectant concentrations that are needed to obtain and maintain the glass-like solidification. Cell shrinkage caused by non-permeable cryoprotectants and the incomplete penetration of permeable components may cause a relative increase of intracellular concentration of macromolecules that is enough to hamper intracellular ice formation. On the other hand, the effect of serious deformations as the consequence of the osmotic effect is most probably less harmful to oocytes and embryos than supposed earlier.

Apart from the total elimination of ice crystal induced injury, another positive feature of vitrification is the considerable decrease of chilling injury, as - in contrast to traditional freezing the high cooling and warming rate at vitrification allows the sample to pass rapidly through the harmful temperature zones (Fig. 5.1).

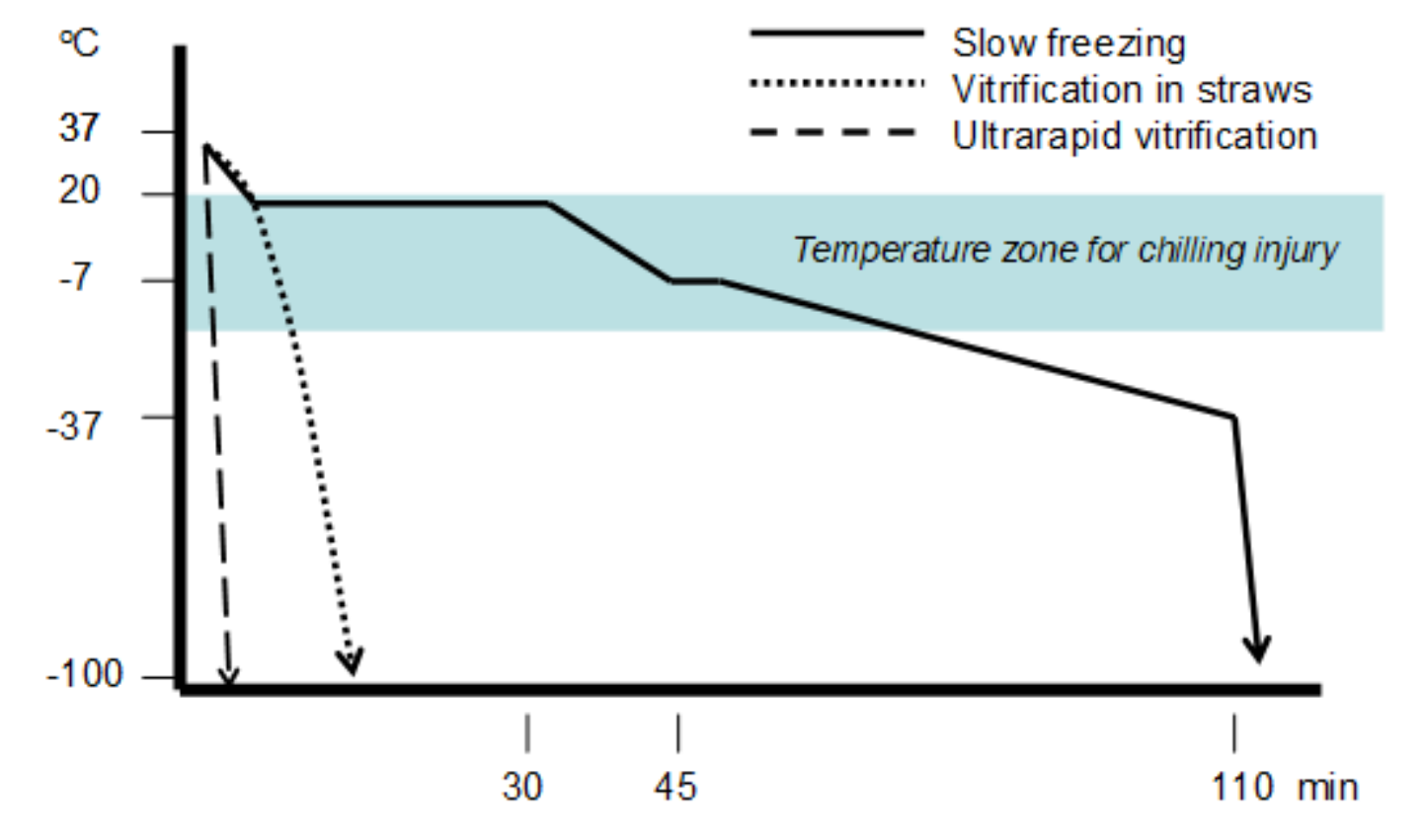

Figure 5.1: Cooling rates during slow freezing, vitrification in straws and ultrarapid vitrification (for the latter, see explanation later). Note the time oocytes / embryos may spend in the temperature zone for chilling injury during the different procedures.

Risks for damage at using the two approaches are summarized in Table 5.2. Both techniques offer positive and negative features, and it is the actual situation (species, developmental phase, origin, purpose, etc.) that determine which approach offers higher chances for optimal survival. 
Table 5.2: Sources and potential degrees of injuries during slow freezing and vitrification

\begin{tabular}{|l||c|c||}
\hline & Slow freezing & Vitrification \\
\hline \hline Chilling injury & +++ & + \\
\hline \hline Ice crystal formation & +++ & - \\
\hline \hline Toxic injury & + & +++ \\
\hline \hline Osmotic injury & + & +++ \\
\hline \hline Fracture injury & ++ & ++ \\
\hline
\end{tabular}

\subsection{The past and the present: slow freezing}

The first successes in mammalian embryo cryopreservation have been achieved with slow freezing (Table 5.1). Apart from the experimental application for mouse embryos, the method was also found efficient for cryopreservation of bovine embryos derived by the MOET (multiple ovulation and embryo transfer) procedure. Another decisive achievement was the successful freezing of early stage human IVF (in vitro fertilized) embryos. Controversially, the need to apply a highly accurate and reliable -consequently complicated and precious - controlled rate freezer was not a drawback, rather a vantage. The industry has realized soon the commercial potential of the method, microprocessor-controlled, liquid crystal display-equipped tools were on the market when most researchers used still mechanical typewriters, ready to use freezing and thawing solutions were offered, and cryopreservation has become soon the most standardized (and most expensive) part of embryology work.

The work of the embryologist was restricted to follow strictly the manuals. Equilibration with the cryoprotectants was performed under a stereomicroscope in a Petri dish, then embryos were loaded into a straw, and after sealing these straws were placed into the freezing machine. The remaining work was almost entirely performed by the machine, except for seeding, although some more sophisticated equipment offered automatic solution for this step, either. At thawing, straws were shortly hold in air (to avoid fracture damages caused by the extremely rapid warming and pressure changes inside the straw, then submerged for several seconds into a water bath close to the core temperature of the mammal. Finally, the content of the straw was poured into a Petri dish containing an osmotic buffer (usually sucrose) to prevent swelling caused by the intracellularly accumulated cryoprotectant; then, according to the rate of diffusion of the cryoprotectant embryos were transferred 
into more and more diluted sucrose solutions, eventually into isotonic medium before transfer or further in vitro culture (Table 5.3).

Table 5.3: A typical slow freezing and tawing method. The method was suggested for cryopreservation of human blastocysts (Van den Abbeel et al., 2005)

\begin{tabular}{|c|}
\hline 1. Blastocysts in Hepes medium for $10 \mathrm{~min}$ at $22^{\circ} \mathrm{C}$ \\
\hline 2. Blastocysts in Hepes medium $-5 \%(\mathrm{v} / \mathrm{v})$ glycerol for $10 \mathrm{~min}$ at $22^{\circ} \mathrm{C}$ \\
\hline $\begin{array}{l}\text { 3. Blastocysts in Hepes medium }-9 \%(\mathrm{v} / \mathrm{v}) \text { glycerol }-0,2 \mathrm{~mol} / \mathrm{l} \text { sucrose } \\
\text { for } 10 \mathrm{~min} \text { at } 22^{\circ} \mathrm{C}\end{array}$ \\
\hline 4. Cooling to $-7^{\circ} \mathrm{C}$ at $2^{\circ} \mathrm{C} / \mathrm{min}$ \\
\hline 5. Seeding with liquid nitrogen (LN2) cold forceps \\
\hline 6. Cooling to $-37^{\circ} \mathrm{C}$ at $0,3^{\circ} \mathrm{C} / \mathrm{min}$ \\
\hline 7. Cooling to $-150^{\circ} \mathrm{C}$ at $50^{\circ} \mathrm{C} / \mathrm{min}$ \\
\hline 8. Storage in LN2 \\
\hline 1. Remowe straws from LN2 and keep for $20 \mathrm{sec}$ at $22^{\circ} \mathrm{C}$ \\
\hline 2. Shake straws for $20 \mathrm{sec}$ in $30^{\circ} \mathrm{C}$ waterbath \\
\hline 3. Empty straw in Petri dish \\
\hline 4. Blastocysts in Hepes medium $-0,5 \mathrm{~mol} / 1$ sucrose for $10 \mathrm{~min}$ at $22^{\circ} \mathrm{C}$ \\
\hline 5. Blastocysts in Hepes medium $-0,2 \mathrm{~mol} / \mathrm{l}$ sucrose for $10 \mathrm{~min}$ at $22^{\circ} \mathrm{C}$ \\
\hline 6. Blastocysts in Hepes medium $-0,1 \mathrm{~mol} / 1$ sucrose for $10 \mathrm{~min}$ at $22^{\circ} \mathrm{C}$ \\
\hline 7. Blastocysts in Hepes medium for $10 \mathrm{~min}$ at $22^{\circ} \mathrm{C}$ \\
\hline 8. Blastocysts in Hepes medium at $37^{\circ} \mathrm{C}$ (repeat $\left.2 \mathrm{x}\right)$ \\
\hline 9. Culture in blastocyst medium \\
\hline
\end{tabular}

One cannot underestimate the significance of this achievement. In a few years, storage and transportation of bovine embryos has become from a laboratory dream a reality and a prosperous business providing entirely new perspectives for rapid improvement of the stock and worldwide propagation of valuable genetic material. In human reproduction, the advancement was more cautious, but during the past two decades transfer of frozen early stage human embryos has become routine procedure in all countries where the actual law permits it. According to the publications of the leading groups in the field, pregnancy rates with frozen of both in bovine and human were approximately $10 \%$ lower than those achieved without freezing: a difference that is difficult to eliminate and easy to accept, considering the benefits the method offers for the cattle industry and human reproduction.

During the past 25 years, the only significant change in this field was the invention of direct transfer in cattle. Embryos frozen in the standard $0.25 \mathrm{ml}$ straw were just thawed in a water bath, then 
the dilution was performed by shaking the sealed straw and unifying the pre-loaded osmotic buffer solution with the embryo containing medium before the transfer (Leibo, 1984). By replacing earlier cryoprotectants with highly permeable and less toxic chemicals, for example ethylene glycol, the efficiency of the procedure has increased significantly. This practical innovation has reduced considerably (from approx. $25 \mathrm{~min}$ to $1-2 \mathrm{~min}$ ) the time between thawing and transfer, did not require the use of a stereomicroscope and has made the method less sensitive to the environment by reducing the possibility of cooling or infection under on-field conditions.

A strange effect of the rapid success of slow freezing was that it has stopped the advancement at least for large-scale practical application. The convenient and safe work, the application of kits and machines, and the lack of need of any knowledge in cryobiology decreased the creative ability of embryologist practitioners and narrowed their openness towards new technologies. On the other hand, the limits of slow-rate freezing have been discovered rather soon. In domestic animals, the successes achieved in bovine in vivo derived embryos could be more or less repeated in ovine and caprine embryos, but almost zero success was reported in swine. Survival and pregnancy rates were high when embryos were in vivo derived, but rather compromised after in vitro embryo production. Moreover, the success rate with early stage embryos was miserable, and slow freezing was almost entirely inappropriate for cryopreservation of oocytes, both before and after in vitro or in vivo maturation in any mammalian species except for the mouse.

\subsection{The future: vitrification}

Compared to the "high technology" image of slow freezing, vitrification is an extremely simple procedure. There is no need for expensive equipment; the whole procedure can be performed in a foam box filled with liquid nitrogen. After equilibration with the cryoprotectant solutions, the sample is simply submerged into the liquid nitrogen. For warming, the principles are the same as after slow rate freezing: a short period in air, then immersion into a warm solution and dilution of the cryoprotectants.

Vitrification has been first used in embryology approximately a decade after traditional slow freezing, and was regarded for long as curiosity of experimental embryologists, without any perspectives for practical application. The narrow gap between pregnancy rates achieved with frozen bovine and human embryos did not necessitate the introduction of a new technology. The frighteningly high cryoprotectant concentrations that are required by early vitrification protocols affrayed practitioners, and the industry was anything but enthusiastic to propagate a method that could be performed without any investment. On the other hand, vitrification in these years could not 
achieve impressing breakthrough in other important areas including cryopreservation of human and domestic animal oocytes, early embryos in domestic species and transferable stage porcine embryos.

To prove the viability and eventually the superiority of vitrification compared to slow freezing, a completely new approach was required. Instead of dealing with the cryoprotectant composition, concentration, addition and dilution, researchers started to focus to the alternative resource: to increase cooling and warming rates. Previously all vitrification work was performed in the traditional tools of cryopreservation: $0.25 \mathrm{ml}$ standard insemination straws and cryovials. These tools were not designed and not suitable to achieve high cooling and warming rates: the maximum achievable cooling rate in a sealed insemination straw is approx. 2000 to $2.500^{\circ} \mathrm{C} / \mathrm{min}$ (Palasz and Mapletoft, 1996; Vajta et al., 1998a), and much lower for cryovials (Fig. 5.1). In practice, however, the maximal cooling rate in closed straws containing solution columns and gas bubbles frequently resulted in fracture damage, or even explosion of the whole straw, destroying all the oocytes or embryos inside, so security measures were applied including pre-cooling the straws in the vapor of liquid nitrogen before submerging, and keeping the straw for a relatively long period of time in room temperature air before immersing it into water bath. However, these manipulations decreased further the speed of cooling and warming.

Theoretically there are three ways to achieve high cooling rates: 1.) to minimize the thermoinsulating layer between the sample and the liquid nitrogen, 2.) to decrease the volume of the solution containing the sample, and 3.) to minimize the liquid vapor formation around the sample.

Regarding the third possibility, it should be noted that at minus $196^{\circ} \mathrm{C}$ liquid nitrogen is at its boiling point, and anything that is warmer and immersed into it induces an extensive evaporation. Accordingly, at vitrification a vapor coat is formed around the sample, and this thermo-insulating steam sheath decreases considerably the cooling rate. One way to avoid this phenomenon is to immerse a metal block into the liquid nitrogen, and to drop the embryo-containing solution onto its approximately $-150^{\circ} \mathrm{C}$ surface (solid surface vitrification; Dinnyés et al., 2001). The other way is to cool the liquid nitrogen below the boiling point, by using a simple law of physics: establishing a vacuum in the container. Part of the liquid nitrogen evaporates, and the remaining part cools down, may even form a slush at $-205^{\circ} \mathrm{C}$. When the sample is immersed into this supercooled nitrogen, the evaporation is minimal and the cooling rate increases. A commercially available equipment has also been produced for this manipulation (Arav et al., 2000).

So far, however, most modern vitrification methods use the other ways to increase cooling and warming rates: they minimize or eliminate the thermoinsulating layer between the liquid nitrogen and the sample, and decrease the quantity of the embryo containing medium. The simplest way and the earliest attempt was to drop the embryo-containing solution directly into the liquid nitrogen (Landa 
and Tepla, 1990). However, although this method may be simple, it is probably not optimal. The required amount to form a drop is relatively high, approximately $5 \mu 1$. Moreover, as the result of the evaporation, the drop floats for a relatively long period of time on the surface of the liquid nitrogen before plunging, and this phenomenon decreases again the cooling rate.

To overcome this problem a carrier tool is required. It is quite impressing that after initiation of the procedure, how much creativity embryologist demonstrated regarding the creation of the appropriate tool. The first successful approach included a small copper grid that is normally used to hold electron microscope sections (Martino et al., 1996b). After initial successes in insects, the method was first applied for bovine oocytes, then for wide variety of mammalian egg cells and embryos including human. As the excess solution is removed from the grid by touching a filter paper, there is only a thin layer of medium around the sample at vitrification and warming. Surprisingly this layer fixes safely the sample to the grid during cooling and storage, but the samples can be removed easily and without any danger of mechanical damage after warming. The only problem of the method was the rather small tool that required delicate handling, and also the difficulty to find an easy and safe method for storage.

The subsequent approach was the open pulled straw (OPS), i.e. a thin and thin-walled plastic straw that is produced by heating a normal insemination straw and pulling it, just like at production of a glass capillary (Vajta et al., 1998a). The open end and the slight capillary effect allowed simple loading and also dilution and expelling, making the tool practical to use. The small (less than $1 \mu \mathrm{l}$ ) solution loaded to the narrow end contacted directly the liquid nitrogen at immersing, accordingly the achievable cooling and warming rates were approximately $20.000^{\circ} \mathrm{C} / \mathrm{min}$, with an order of magnitude higher than those at the original straws (Table 5.4). A special benefit of the OPS was that it allowed microscope free, semidirect transfer in cattle by immersing the narrow end into a standard $0.25 \mathrm{ml}$ straw filled with a warm sucrose medium: without any further manipulation, embryos could be transferred immediately into the recipient animals using the standard methods. 
Table 5.4: OPS vitrification protocol for bovine embryos (Vajta et al., 1998a)

\begin{tabular}{|c|}
\hline Equilibration and cooling \\
\hline $\begin{array}{l}\text { 1. Incubation in holding medium (Hepes buffered T199 medium }+20 \% \text { cattle serum) at } 37^{\circ} \mathrm{C} \text { for } \\
5 \mathrm{~min}\end{array}$ \\
\hline $\begin{array}{l}\text { 2. Equilibration in HM containing } 7.5 \% \text { ethylene glycol and } 7.5 \% \\
37^{\circ} \mathrm{C} \text { for } 3 \mathrm{~min}\end{array}$ \\
\hline $\begin{array}{l}\text { 3. Equilibration in } \mathrm{HM} \text { containing } 16.5 \% \text { ethylene glycol, } 16.5 \% \text { dimethylsulphoxide and } 0.7 \\
\mathrm{M} \text { sucrose at } 37^{\circ} \mathrm{C} \text { for } 1 \mathrm{~min} \text {. Loading to OPS } \quad \text { during this period }\end{array}$ \\
\hline 4. Immersion of the narrow end of OPS containing the sample into liquid nitrogen \\
\hline Warming and dilution \\
\hline $\begin{array}{l}\text { 1. Immersion of the narrow end of OPS into } 37^{\circ} \mathrm{C} \text { holding medium containing } 0.6 \\
\text { sucrose. Expelling embryos from the straw. Incubation for } 5 \text { min. }\end{array}$ \\
\hline 2. Transfer to $37^{\circ} \mathrm{C}$ holding medium containing $0.3 \mathrm{M}$ sucrose. Incubation for $5 \mathrm{~min}$. \\
\hline 3. Transfer to $37^{\circ} \mathrm{C}$ holding medium, incubation for $5 \mathrm{~min}$. \\
\hline 4. Transfer to culture medium and in vitro culture. \\
\hline
\end{tabular}

It is hard to determine how many "clones" of the OPS method have been developed during the past 10 years, including glass micropipettes (Kong et al., 2000), super-finely pulled OPS, (Isachenko et al., 2000), gel-loading tips (Tominaga and Hanada, 2001), sterile stripper tip (Kuleshova and Lopata, 2002), flexipet denuding pipettes (Liebermann et al., 2002a), fine diameter plastic micropipettes (Cremades et al. 2004), $100 \mu$ l pipetting tip (Hredzak et al., 2005), closed pulled straws (Chen et al., 2001) sealed open pulled straws (Lopez-Bejar and Lopez-Gatius, 2002), and finally a more professional and sophisticated form, the Cryotip system (Kuwayama et al. 2005b). Except for the latter developed specially for human use, none of them has clearly proved its superiority compared to the original tool. In contrast: the OPS is still a method of choice for many applications where routine cryopreservation of relatively large quantities are required, especially in animal biotechnology.

Other tools based on a different approach include the Cryoloop, a thin plastic loop, where the embryos are loaded on the film bridging the loop and formed from the cryoprotectant medium. As demonstrated recently, the extremely small amount of medium allows successful vitrification also in the vapor of liquid nitrogen. Another group of techniques is called the Minimum Drop Size or Minimum Volume Cooling method or the hemi-straw system (Arav, 1992; Hamawaki et al., 1999; Vanderzwalmen et al. 2000) consisting of a small droplet of vitrification solution containing the oocyte or embryo placed on a solid surface that is immersed into liquid nitrogen. A sophisticated and practical version of this procedure has been introduced later by Kuwayama et al. (2000) with the Cryotop method, where the tool is a thin plastic film attached to a solid plastic holder and also 
equipped with a protective cap for the film part to protect the sample from mechanical injuries during storage.

So far, no comprehensive study has been performed to compare the efficiency of vitrification performed with the individual tools listed above. As most of them are suitable to achieve or surpass the approximately $20.000^{\circ} \mathrm{C} / \mathrm{min}$ cooling rates that seems to be critical to avoid chilling and to allow a considerable decrease in the required concentration of cryoprotectants, practicality and safety are theoretically the points of selection. However, at present most embryologist learn only one (if any) vitrification method, and use it for all purposes without any attempt to change. Everybody claims the given method practical and safe, and disregards the potential benefits the alternative approaches may offer.

Eventually, however, a kind of standardization should occur regarding the tools as well as solutions, cooling and warming rates, and equilibration-dilution parameters. Here the commercial contribution may not be a real benefit as the more sophisticated, consequently more expensive and profitable tools (that are usually supported by the industry) aren't necessarily the optimal ones. The other external factor is the legislation that has hampered seriously the application -especially human application - of vitrification, so far.

\subsection{Disease transmission mediated by the liquid nitrogen}

The main concern of authorities is the danger of liquid nitrogen mediated biological contamination of samples. As mentioned earlier, viruses and bacteria may survive cooling, storage at $-196^{\circ} \mathrm{C}$ and warming without any cryoprotectants. Evidently, their survival chances increase with the use of sophisticated freezing and vitrification techniques. Unfortunately some kind of release from the solid sample blocks and free circulation in the liquid nitrogen may occur anytime in a liquid nitrogen tank, and eventually cross-contamination between samples may occur (. Additionally, sterilization and aseptic handling of containers is technically impossible - the outer surface of all cryovials and straws is infected and means a source of contamination.

However, most probably due to the negligible biological activity at $-196^{\circ} \mathrm{C}$, disease transmission caused by liquid nitrogen-mediated contamination are extremely rare. The few documented cases have occurred in humans when large samples such as blood containers were stored together, and leaks occurred in both the source and the recipient container. The volume of these samples with 105 to 106 times higher than those used by the open vitrification methods. On the other hand, the supposedly safe (and officially approved) ways of cryopreservation may not be as safe as supposed walls of traditional staws used for slow freezing of sperm and embryos are not impermeable for bacteria and viruses, either, and leaks may always occur as the result of inappropriate sealing. However, no disease 
related to cross contamination mediated by the liquid nitrogen has been reported in human and animal embryology yet. The potential danger of infection is far lower than that imposed by a standard face mask at surgical procedures: however, surgeons were not suggested to wear ventilated airproof suits and helmets (the issue is reviewed in detail in Vajta and Nagy, 2006).

However, vitrification techniques may meet the highest standards of sterility, either. Factoryderived, sterile filtered liquid nitrogen can be aseptically stored and used for the cooling procedure. Subsequently samples and carrier tools (OPS, Cryotop) can be hermetically wrapped into precooled larger $(0.5$ or $1.0 \mathrm{ml})$ straws specially developed for sterile storage and sealed with approved leakproof methods (Vajta et al., 1998b). All techniques and tools are available for these procedures, and should be considered when the expected standardization of vitrification in domestic animal and human embryology gets realized.

\subsection{Additional approaches to improve cryopreservation results}

Apart from the refinement of the basic procedures (including adjustment of cooling and warming rates, composition of media and cryoprotectant concentration and introduction of new carrier and storage tools) there are other possibilities to improve survivals of embryos and oocytes after cryopreservation. Here we list only three possible ways: removal of the lipids, induced collapse of the blastocoel and high hydrostatic pressure treatment.

Chilling injury of lipid droplets during cryopreservation is the major cause of damage and death in organisms that are intrinsically rich in these structures including porcine oocytes and embryos. The function of these lipid droplets, and especially the reason of their extreme abundance in some species is unknown. The hypothesis that they serve as an energy source seems to be unsupported by the fact that oocytes and embryos in other species (mouse, human, rabbit) can survive well with orders of magnitude less lipid; and removal of these lipid droplet does not hamper developmental competence in cattle and pigs.

Due to the high number and almost equal distribution in the cytoplasm the removal requires bulk methods. So far, the only efficient procedure for the purpose was the high-speed centrifugation (up to $13.000 \mathrm{G}$, for 15 to $20 \mathrm{~min}$ ) to separate lipids with lower buoyant density from the rest of the cytoplasm. However, even with this extreme procedure a full separation was not achieved and a narrow cytoplasmic bridge connected the lipids to the remaining part. This bridge widened in several hours after centrifugation and lipids were incorporated again. Accordingly, the procedure was only successful when accomplished with mechanical removal of the lipid fraction with the use of micromanipulators. Although considerably increased survival rates were achieved, the delicate and labor-intensive procedure hampered large-scale practical application (Nagashima et al., 1994). 
Recently, Li et al. (2006a) reported birth of the first piglets after vitrification of somatic cell nuclear transferred embryos produced from delipated oocytes by using micromanipulation both for delipation after centrifugation and nuclear transfer. However, the authors still emphasized the need to develop less labor-intensive procedures.

A simplified approach has been described to perform a bulk enucleation method that can be used both for oocytes and embryos. The zona pellucida is partially digested before centrifugation to enlarge the perivitelline space. This enlarged space allows sufficient elongation during centrifugation to separate completely lipid droplets from the remaining cytoplasm. After a subsequent full digestion of the zona, the transparent globes of lipid droplet-free cytoplasm can be easily separated from the dark agglomerated lipid droplets, and the former can be used for either further in vitro culture directly (in case of embryos) or nuclear transfer (in case of oocytes). Blastocysts arising from the delipated oocytes or embryos have higher cryotolerance and may develop to live offspring after vitrification (Esaki et al., 2004; Du et al., 2007).

In human embryology, another problem required different kind of manipulation to increase cryotolerance. The purpose of human assisted reproductive techniques is to achieve the highest pregnancy rates but to avoid multiple pregnancies that may cause complications at birth. During the past decade impressing advancement has been achieved towards this goal with in vitro culture techniques resulting in high blastocyst rates, and subsequent single blastocyst transfer. However, cryopreservation of blastocysts in humans (in contrast to domestic species) is less efficient at the blastocyst stage than that of cleavage stage embryos. Although new vitrification methods have resulted in improvement towards this goal (Kuwayama et al., 2005b), some researchers suggest artificial collapse of the blastocoel by needle puncture, micropipetting or laser pulse (Vanderzwalmen et al., 2002; Hiraoka et al., 2004; Mukaida et al., 2006). The supposed mechanism may be a decreased toxic effect of the cryoprotectants that is dissolved in the blastocoelic fluid. Very recently, a similar effect has been observed in porcine blastocysts after collapse induced by mechanical puncture (Lin et al., in press).

Very recently, accumulating evidences prove that a surprising pre-treatment results in improved cryosurvival rates. Oocytes and embryos exposed to high hydrostatic pressures (200 to 800 times higher than the normal atmospheric pressure) for 30 to 60 min induce changes in the oocytes and embryos that eventually results in higher survival rates at cryopreservation several hours after pressure treatment. The applied sublethal dose of high hydrostatic pressure may induce the production of heat shock proteins that play an essential role in mammalian embryo development (Esfandiari et al., 2007). Pressure pre-treatment has resulted in significant increase in cryotolerance in mouse and bovine embryos as well as in porcine oocytes (Pribenszy et al., 2005a; b; Du et al., in press). 


\subsection{Results achieved by vitrification}

Vitrification has considerably expanded application fields of cryopreservation in reproductive biology. Achievements during the past 10 years have been summarized on Table 5.5. Additionally, more than 400 papers were published about vitrification during this period, and most of them proved improved survival and developmental rates compared to those achieved with slow freezing. It is quite surprising that this waste amount of evidence is still insufficient to change the mind of domestic animal and especially human embryologist. Up till today, vitrification is still regarded as "experimental", "emerging" technology "withot evidence of harmlessness". Controversially, most respected scientist who emphasize these concerns were decades ago the forerunners of slow freezing, a method without any evidence of harmlessness (that time) and (up till today) without much basic scientific knowledge regarding the ongoing processes.

If we approach the situation without any prejudice, it is quite evident that vitrification has much more potential than exploited, and sooner or later will mostly or entirely replace slow freezing in embryology. The rate of this change depends on the rate embryologist acknowledge its value and learn its proper application.

Table 5.5: Examples in mammalian embryology where first success in cryopreservation was achieved by vitrification. Embryos and oocytes were not treated mechanically or chemically to prepare them for the vitrification. Full term developments were reported except for otherwise indicated (Vajta and Nagy, 2005, modified).

\begin{tabular}{|l|l|}
\hline Species, stage, system & Reference \\
\hline \hline Bovine immature oocytes for IVF & (Vieria et al., 2002) \\
\hline \hline Bovine in vitro matured oocytes for IVF & $\begin{array}{l}\text { (Martino et al., 1996b; Vajta et al., } \\
\text { 1998a) }\end{array}$ \\
\hline $\begin{array}{l}\text { Bovine in vitro matured oocytes for somatic cell nuclear } \\
\text { transfer }\end{array}$ & (Hou et al. 2005) \\
\hline \hline Bovine cytoplasts for embryonic cell nuclear transfer & (Booth et al., 1999) \\
\hline \hline Bovine early stage IVF embryos & (Vajta et al., 1998a; in vitro study) \\
\hline \hline $\begin{array}{l}\text { Bovine zona-included blastocysts generated by somatic } \\
\text { cell nuclear transfer }\end{array}$ & (French et al., 2002) \\
\hline \hline $\begin{array}{l}\text { Bovine zona-free blastocysts generated by somatic cell } \\
\text { nuclear transfer }\end{array}$ & (Tecirlioglu et al., 2004) \\
\hline \hline $\begin{array}{l}\text { Bovine transgenic blastocysts generated by somatic cell } \\
\text { nuclear transfer }\end{array}$ & (French et al., 2003) \\
\hline \hline $\begin{array}{l}\text { Ovine zona included embryos generated by nuclear } \\
\text { transfer }\end{array}$ & (Peura et al., 2003) \\
\hline \hline Porcine immature oocytes for ICSI & (Fujihira et al., 2004; in vitro study) \\
\hline \hline Porcine in vitro matured oocytes for ICSI & (Fujihira et al., 2005; in vitro study) \\
\hline \hline
\end{tabular}




\begin{tabular}{||l|l||}
\hline Porcine in vivo derived blastocysts & (Kobayashi et al., 1998) \\
\hline \hline Porcine in vivo derived morulae & (Berthelot et al., 2001) \\
\hline \hline Porcine in vitro produced blastocysts & (Men et al., 2005; in vitro study) \\
\hline \hline Equine in vivo matured oocytes & (Maclellan et al., 2002) \\
\hline \hline Siberian tiger in vivo derived embryos & (Crichton et al., 2003; in vitro study) \\
\hline \hline European polecat in vivo derived morulae and blastocysts & (Piltty et al., 2004) \\
\hline \hline Rabbit pronuclear stage zygotes & (Hochi et al., 2004) \\
\hline \hline Minke whale immature oocytes for maturation & (Iwayama et al., 2004; in vitro study) \\
\hline \hline Mouse preantral ovary follicles & (Kagawa et al., 2007) \\
\hline \hline Red deer in vivo produced embryos & (Soler et al., 2007) \\
\hline
\end{tabular}
Abbreviations
ICSI - Intracytoplasmic sperm injection
IVF - In vitro fertilization
OPS - Open Pulled Straw vitrification method 


\section{Embryonic genome activation}

\subsection{Introduction}

Sexual reproduction involves the formation of a new functional genome from two different cells, the paternal and maternal gametes, which have evolved separately and differently up to the point of fertilization. In many animals, gametes are transcriptionally inactive at fertilization, and development is initiated under the control of maternal factors stored during oogenesis. In Drosophila and Xenopus, mRNAs stockpiled in the oocyte are stable after fertilization and regulate many aspects of embryonic development (Wolpert, L., et al., 2002). Conversely, in mammals, oocyte-derived mRNAs are degraded shortly after fertilization and cannot direct more than the first few cell divisions. Therefore, embryonic genome activation must occur very early during mammalian development (Schultz, R.M., 2002; Thompson, E.M. et al., 1998). Activation is required for continues development because mRNAs common to the oocyte and embryo (e.g., genes involved in the maternal to embryonic transition and housekeeping genes) are replenished and many genes not transcribed in the oocyte are expressed for the first time. The formation of embryonic genome and the establishment of its own control over the developmental processes involve both the activation of transcription and the coordinated expression of specific genes in a defined chronological order. The outcome of the genome activation is a novel gene expression profile that establishes the totipotent state of each blastomere in the cleavage-stage embryo. This step is a prerequisite for future cell lineage commitments and differentiation events that underlie pattern formation and organogenesis.

The activation of embryonic genomes is characterized by three phases (Thompson, E.M. et al., 1998):

1. The acquisition of a transcriptionally permissive state during the first $S$ phase(s);

2. The onset of a minor genome activation phase initiated in some species already in 1cell embryo and lasting over one or several cleavage cycles;

3. A major genome activation, that in all mammalian species studied, is coincident with the transition from maternal to embryonic control of development.

Embryonic genome activation (EGA) follows as a multiple-step event instead of a single global transcriptional switch. Most genes are transcribed in a stage- and time-dependent manner following the common maternal and/or embryonic expression pattern. In mammals, including humans, the embryonic genome is activated in a similar stepwise manner at the 2 to 4 -cell stage, 4 to the 8 -cell stage or 8 to the 16-cell stage. Genes preferentially transcribed are associated with developmental 
competence and are known to be involved in the regulation of transcription and translation, posttranslational modification of proteins, cell cycle regulation, folliculogenesis, (oxidative) stress defense, histone composition, gap junction signaling, prostaglandin synthesis, growth factor and cell signaling (including receptors), extracellular matrix degrading components, metabolism, and transport system.

\subsection{Inactive creates active}

The mature oocyte shuts down transcription by maintaining its chromatin in metaphase II state. Instead of delivering genetic information as a set of metaphase II chromosomes, however, the sperm arrives in a transcriptionally inert state through the packaging of DNA in specialized basic proteins, including various protamines and histone variants.

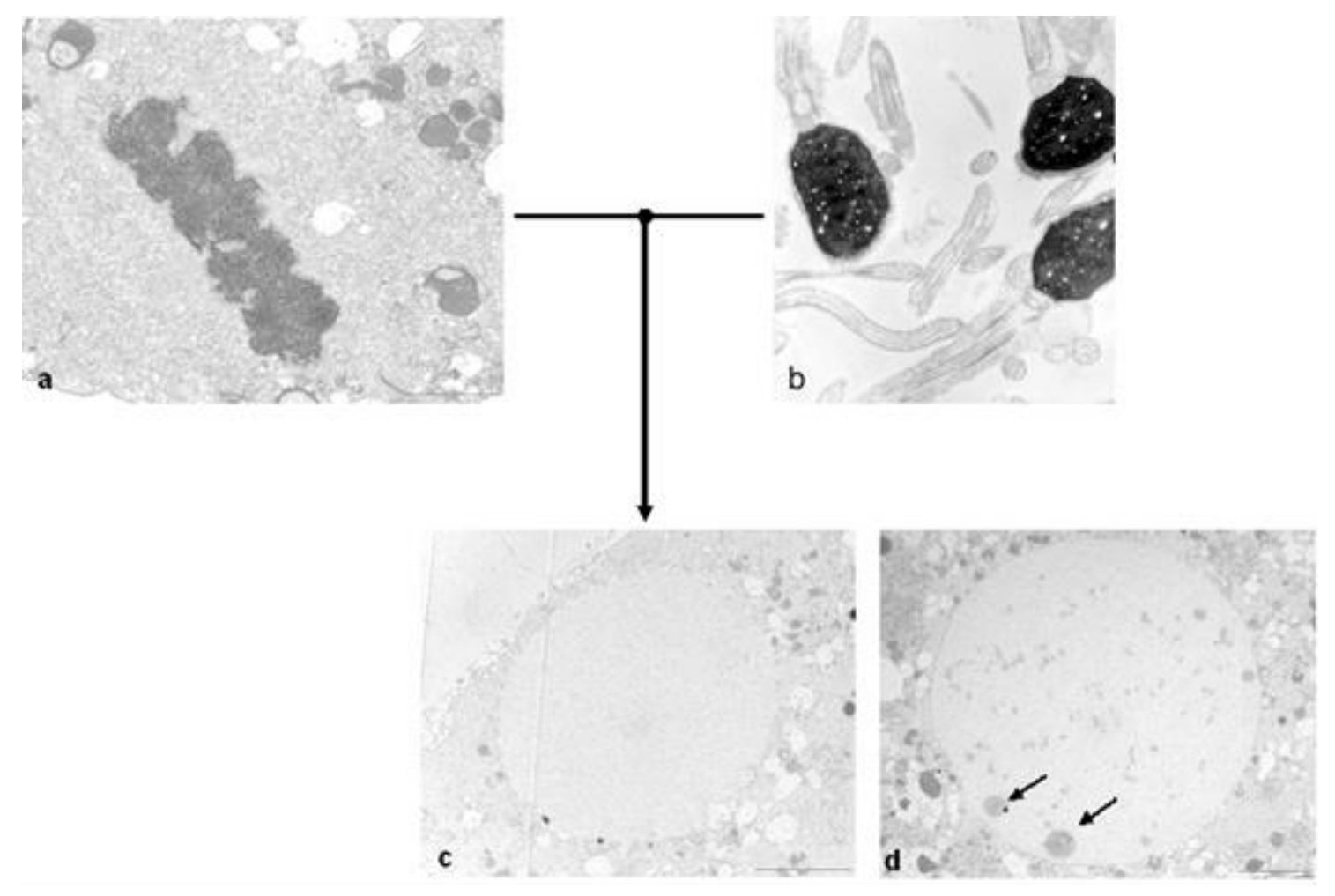

Figure 6.1: Gametes at the time of fertilization contain tightly packed DNA. Oocyte displays chromosome in metaphase II conformation (MII) (a) and sperms have DNA packed together with protamins (b). After fertilization, global chromatin decondenation occurs and results in formation of pronucleus $(c)$, which moves from the periphery to the zygote center and develop functional nucleoli - $\operatorname{arrow}(d)$.

Fusion of the transcriptionally silent gametes at fertilization is followed by a lag period of transcriptional incompetence that last, depending on the species, 20 (mouse)-96 (cow) hours (Latham, 
K.E. et al., 1992). However, very low level of transcription is already observed at cell cycle prior to cycle when major genome activation occurs, and it is called minor genome activation.

Transcription in mouse is first detected shortly after the initiation of DNA replication, and suggests that DNA replication may be linked to initiation of transcription. Consistent with this is the observation that inhibition of the first round of DNA replication results in a $40 \%$ decrease in transcription (Aoki, F.et al., 1997). The linkage between initiation of transcription and DNA replication may be that the disruption of nucleosomes, which inherently inhibit transcription, during DNA replication may provide a window of opportunity for the maternally derived transcription machinery to gain access to promoters that were previously not accessible. Access to these promoters could provide a mechanism in which the maternally derived transcription machinery could reprogram the pattern of gene expression.

Although the transcription is detected already during the first cell cycle after fertilization in some mammalian species, it is not apparent if these transcripts are efficiently translated. The biological significance of an uncoupling of transcription from translation may be to protect the early embryo from the promiscuous expression of genes that could ensue as a consequence of the dramatic chromatin remodelling events that occur while the maternal and paternal genomes are sculpted into a chromatin structure that is present in following cell cycles. Formation of this mature chromatin structure may be essential to support the appropriate pattern of gene expression required for continued development. The other possibility is that it serves to mark promoters that will be rapidly utilized following cleavage (Schultz, R.M., 2002).

Following the minor genome activation, a complete change in the pattern of protein synthesis occurs during the cleavage period, with a rapid degradation of maternal transcripts. Embryonic transcription is then required for further development and the amount of neosynthesized transcripts increases 5- to 10-fold by the time the first cellular differentiation appears at the blastocyst stage.

\subsection{Maternal-embryonic transition}

The first major developmental transition that occurs following fertilization is the maternal to embryonic transition (MET) in which the developmental program that is initially directed by maternally inherited proteins and transcripts is replaced by a new program as the consequence of the expression of new genes. This transition is also called zygotic gene activation (ZGA) or embryonic genome activation (EGA) and occurs in species-specific developmental stages. 


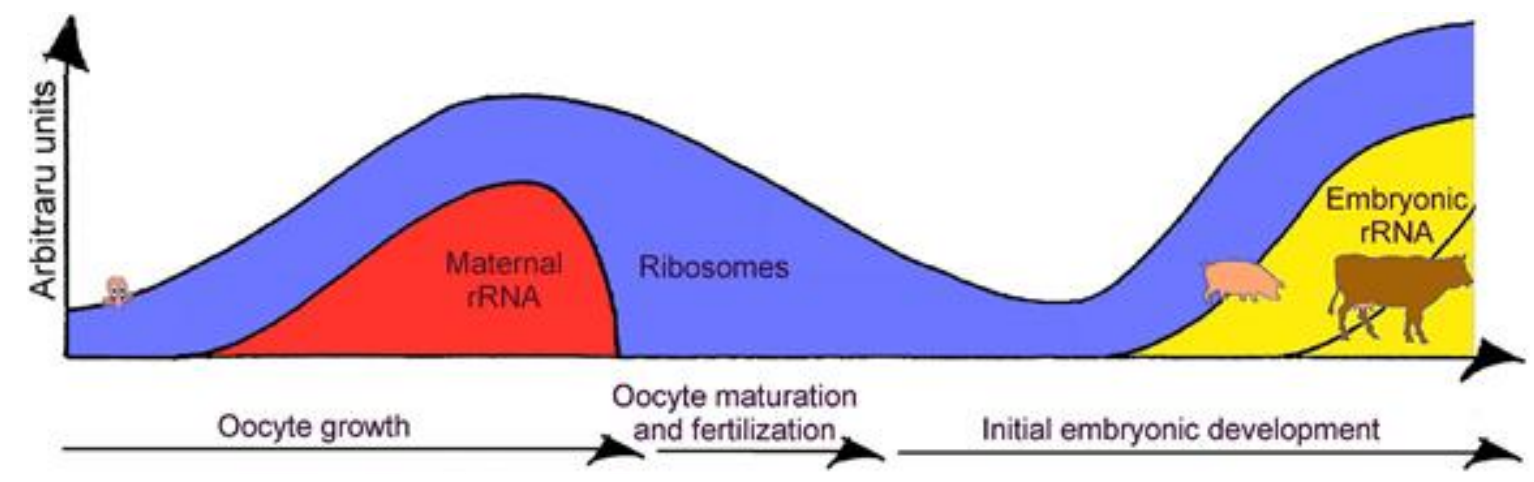

Figure 6.2: Changes in total level of RNA (blue) and levels of maternal rRNA (red) and newly synthesized rRNA (yellow) following the oocyte maturation, fertilization and coming genome activation. Modified according to Maddox-Hyttel.

The EGA has at least three functions that are required for the continued progression of development (Schultz, R.M., 2002).

1. The first function is to destroy oocyte-specific transcripts that are not subsequently expressed. The destruction of these mRNA restricts the period of time in which these genes can function.

2. The second function is to replace maternal transcripts that are common to the oocyte and early embryo, e.g. actin, with embryonic transcripts. If these transcripts are not replaced, it is readily apparent that development will shortly come to a halt due to the inability of the embryo to execute its basic cellular functions. While the expression of these does not result in reprogramming of gene expression in the classical sense, their expression is nonetheless essential.

3. The third function of this transition is to promote the dramatic reprogramming in the pattern of gene expression that is coupled with the generation of novel transcripts that are not expressed in occyte. This reprogramming of gene expression is likely the molecular underpinning for the transformation of the differentiated oocyte into the totipotent blastomeres.

\subsection{Genome activation and cell organization}

Changes during the EGA are not restricted to proteins and RNAs but also affect cellular organelles. Redistribution of mitochondria and endoplasmatic reticulum (ER) has been documented in several organisms (Stitzel, M.L., Seydoux, G., 2007). However, the major ultrastructural morphological changes occur in nucleolar precursors which subsequently develop fully functional nucleolus.

Nucleolar precursors in pre-EGA embryos appear as densely-packed spherical bodies, called nucleolus precursor bodies (NPBs). At the time of the EGA, NPBs start to loose their compact form 
and transform into particular components of functional nucleolus driven by starting activity of ribosomal RNA genes transcription. Until the EGA homogeneous mass of NPBs transforms into fibrillar centers (FCs), dense-fibrillar component (DFC) and granular component (GC), which reflect following steps in rRNA synthesis (FC) and processing (DFC, GC). In some species, e.g. mouse, pig, human, these structures first appear in semilunar structures on the surface of NPBs. In other ones, e.g. cattle, NPBs form primary and secondary vacuoles and on the rim of these, functional nucleolar components can be firstly observed. On cell cycle after the EGA, fully functional nucleoli are found in all blastomeres of developing embryo.
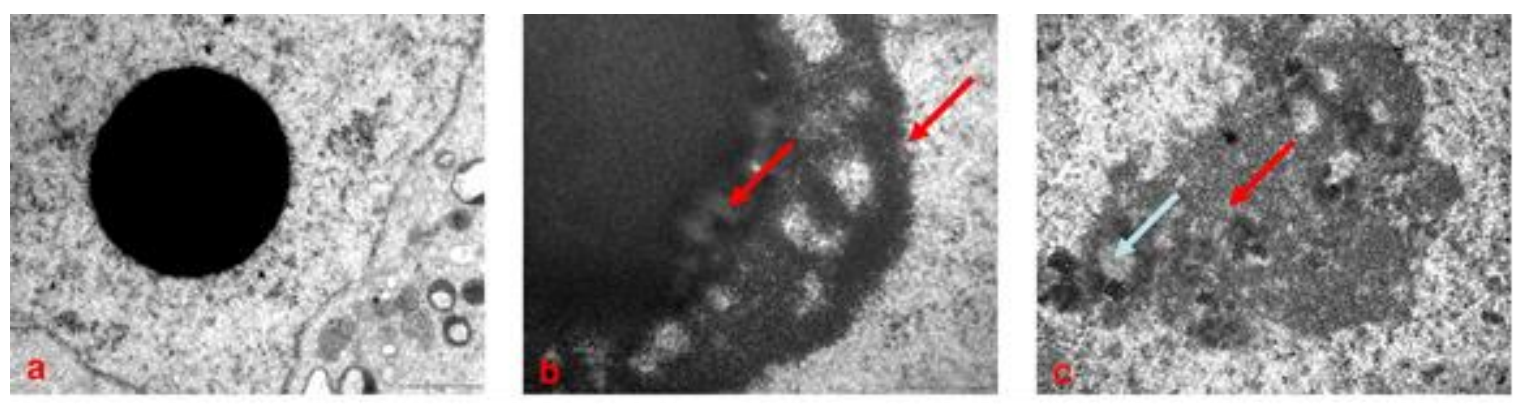

Figure 6.3: Changes occurring during transformation of nucleolar precursor into functional nucleolus. (a) Nucleolar precursor body consists of densely-packed material. During embryonic genome activation, functional components start to develop $(b)$ and in the following cell cycles, nucleoli display fully developed fibrillar centers (blue arrow) surrounded by dense-fibrillar component and granular component (red arrow).

\subsection{Regulation of embryonic genome activation}

\subsubsection{Maternal regulation of early development}

Maternal gene products drive early development when the newly formed embryo is transcriptionally inactive. During this period, overall amount of cytoplasm in the embryo remains constant, the number of nuclei and the amount of DNA increase exponentially and mRNAs and proteins provided to the egg by the mother drive development (Meirelles, F.V.et al, 2004). By contrast, the embryonic genome is transcriptionally activated only at later cell cycle. EGA then coincides with cell cycle lengthening and the degradation of many maternal RNAs.

The maternal mRNAs and proteins reserve in the oocyte decline with time, with a very narrow opportunity for post-transcriptional process and the gradual substitution by embryo transcripts and its translation products. The competent ooplasm have all the mRNA needed for embryonic development to the major genome activation and, thus, for successful remodelling of maternal and paternal genome 
into transcriptionally active embryonic genome (Memili, E., First, N.L., 1999). However, the activation of maternal mRNAs and transcripts still remain to be fully clarified. In many cases, activation depends on liberating mRNAs from complexes that block translation initiation. For example in mouse and Xenopus has been proved, that mRNA is bound by translation-repressing complex preventing the recruitment of the translation initiation factor. During oocyte maturation, elements of the repressing complex phosphorylate and release the mRNA for translation.

Ooplasm carries not only "good" transcripts necessary for following cleavages, but there are also many cell-death gene-transcripts and proteins. The presence of these products is justified by the need of the oocyte to undergo cellular death as follicles become atretic (Meirelles, F.V. et al., 2004). A healthy embryo, therefore, must not only activate its genome in regard to developmentally important genes, but also to maintain the balance of death/survival factors in the cytoplasm.

Whereas zygotic mRNAs are synthesized during embryogenesis, many maternal mRNAs are degraded. Degradation removes gene products that might interfere with later development. This regulated maternal mRNA destabilization is mediated by sequences in the 3'untranslated region. Special proteins and RNAs bind to these sequences and induce the deadenylation of target mRNAs, which are then prone to degradation by proteases.

\subsubsection{Epigenetic modification}

The preimplantation period of mammalian development includes the formation of the zygote, the activation of the embryonic genome, and the beginning of cellular differentiation. During this period, protamines are replaced by histones, the methylated haploid parental genome undergoes demethylation following formation of the diploid zygote, and maternal control of development is succeeded by zygotic control. The aim of these dramatic changes is to transform the highly differentiated oocyte into a totipotent blastomere of the early cleavage stage pre-implantation embryo.

Changes in chromatin structure accompany transcriptional activity of eukaryotic genes. Chromatin structure and nucleosome organization provide architectural linkages between gene organization and the regulatory components of transcriptional control. The basic principles of chromatin organization involve wrapping of DNA around histone octamers to form nucleosomes, the folding of the nucleosomal fiber into higher-order structures, and DNA methylation. 

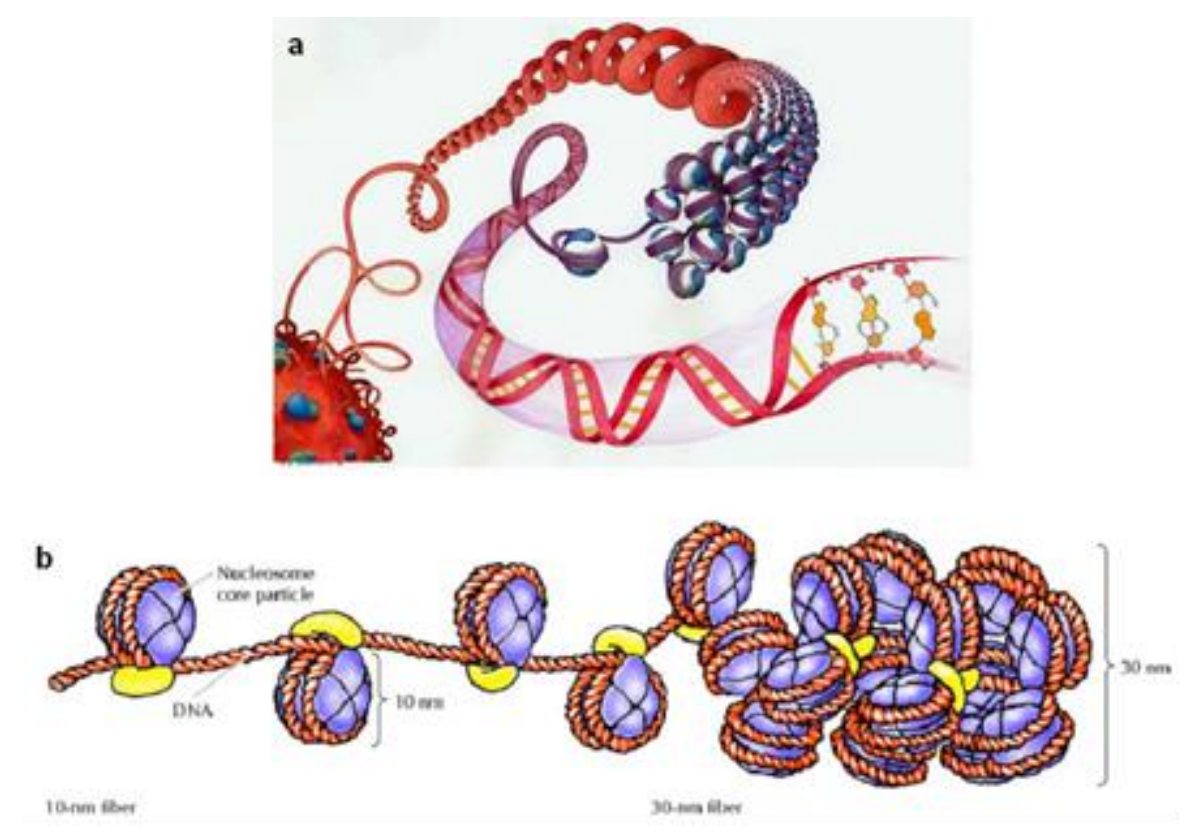

Figure 6.4: Folding of DNA into nucleosomal structures. (a) DNA consists of nucleotides which are connected into two DNA fibers. They create typical helical structures which are stabilized with help of nucleosome core particles (b). This more-level folding results in chromosomal structures.

Two molecules of each of four core histones (H2A, H2B, H3 and H4) wrap 160 bp of DNA and, together with the single molecule of linker histone (H1), organize 180-190 bp of DNA into a nucleosome. The N-termini of the core histones $\mathrm{H} 3$ and $\mathrm{H} 4$ are among the most highly conserved sequences in eukaryotes.

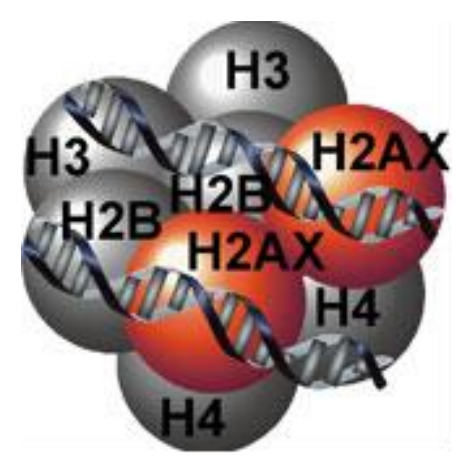

Figure 6.5: Histones $\mathrm{H} 2 \mathrm{~A}, \mathrm{H} 2 \mathrm{~B}, \mathrm{H} 3$ and $\mathrm{H} 4$ present the core around which the DNA is being folded. Two of these structures are connected via linker histone $H 1$.

Post-translational modifications of conserved tail amino acid modulate their interaction potential and together with DNA methylation create the basic epigenetic modifications regulating accessibility of transcription factors to DNA. 
Epigenetics includes gene-regulating activity that is not directly related to the DNA sequence itself and can persist through one or more generations. Several converging lines of evidence based on the expression of both reporter genes and endogenous genes suggest that the major locus of regulation for the observed repression is mediated by changes in chromatin structure, and not changes in the activity of the transcription machinery per se.

\subsubsection{DNA methylation}

DNA methylation is one of the principal mechanisms by which eukaryotic cells maintain a stable chromatin configuration that represses transcription. DNA methylation occurs on $\mathrm{CG}$ or $\mathrm{CNG}(\mathrm{N}=\mathrm{T}$, $\mathrm{G}, \mathrm{A}$ or $\mathrm{C}$ ) sequences in a reaction catalyzed by the enzyme DNA methyl transferase (Dnmt). The target $\mathrm{CpG}$ dinucleotides are found predominantly in regions of the genome known as $\mathrm{CpG}$ islands, which are sequences of about 200 bp or larger with CG content above 50\%. Undermethylated CpG islands are associated with decondensed chromatin structure and principally distributed within the promotor regions of transcriptionally active genes. Transcriptional suppression by DNA methylation may involve a direct mechanism in which $\mathrm{CpG}$ methylation prevents binding of the basal transcriptional machinery or specific transcription factors that require contact with cytosines in their cognate binding elements.

\subsubsection{Histone modifications}

Post-translational modifications of histones have been implicated in the physiological control of chromatin structure for last three decades. Acetylation of the amino-termini of nucleosomal histones has been directly correlated with transcriptional activation. Hyperacetylation of core histones enhances the binding of most transcription factors to nucleosomes. On the other hand, nuclear histones deacetylation reactions are associated with a condensed chromatin structure and transcriptional repression, indicating that the rate of histone turnover has a key role in modulating gene expression. 


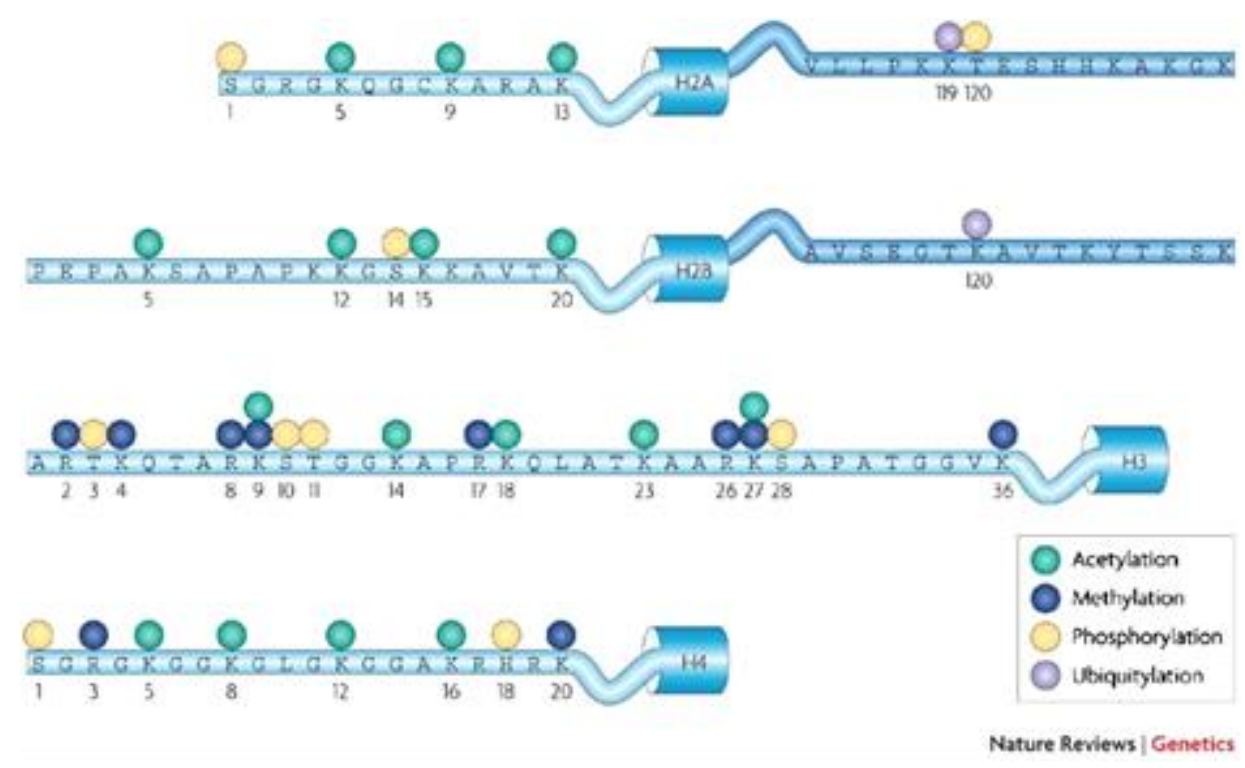

Figure 6.6: Sites of possible histone modifications. Note that $\mathrm{H} 3$ displays the most variable lysine residues for histone modifications.

\subsubsection{Epigenetic changes and reprogramming in early embryo development}

Mammalian development is characterized by bimodal DNA methylation reprogramming that occurs initially during germ cells development and then during preimplantation. Primordial germ cells (PGCs) enter the developing germinal ridge and begin differentiation and expansion. At this time, the highly methylated PGCs undergo rapid genome-wide demethylation and most of the methylation is lost. This reprogramming phase coincides with the erasure and resetting of parent-oforigin specific marks that include DNA methylation of imprinted sequences associated with allelespecific gene expression. Within a few days, de novo methylation appears and establishes the new gamete-like information.

The second phase of reprogramming occurs between fertilization and formation of the blastocyst. At fertilization, the parental genomes are in different stages of the cell cycle with very different epigenetic marks and chromatin organization. The paternal genome has been delivered by the mature sperm, is single copy (1C), and is packaged densely for the most part with protamines rather than histones. In contrast, the maternal genome is arrested at metaphase II with its $2 \mathrm{C}$ genome packaged with histones.

Upon fertilization, protamines in sperm chromatin are rapidly replaced with histones closely followed by a rapid paternal-specific asymmetric loss of methylation. This process takes place in the absence of transcription or DNA replication and is termed active demethylation. This demethylation is finished in male paternal pronucleus before the onset of DNA replication. However, some regions 
of the genome do not become demethylated at this stage. These include heterochromatin in and around centromeres, IAP retrotransposons and paternally methylated imprinted genes.

In maternal pronucleus, demethylation occurs during the following cell cycles without significant activity on the genome. DNA methylation is reduced progressively with cleavage divisions and depends on DNA replication. Indeed, DNA methyl transferase (Dnmt 1) protein inherited from the oocyte (Dnmt1o) is seemingly excluded from the nucleus during the pre-activation cell cycles accounting for the loss of methylation by a passive demethylation. Many different types of sequences are demethylated at this stage but imprinted genes retain their germ-line imprints. The maintenance of imprints is enabled by temporary entry of Dnmt1o into nucleus shortly before or during the genome activation. Dnmt1 is responsible for maintaining of methylation pattern toward the mitotic division copying this pattern from hemimethylated substrate to newly formed DNA string.

The initiation of de novo methylation occurs after the genome activation and is, thus, under the control of embryonic genome. De novo methylation is evident in both cell lineages of the blastocyst. However, it is the inner cell mass which becomes hypermethylated in comparison with the trofectoderm. Genome-wide de novo methylation is carried out by Dnmt3a and Dnmt3b, and is conferred by specialized chromatin structures on the unmethylated allele. In general, genomic sequences are likely to require other epigenetic marks to be protected from, or to attract, de novo methylation stage.

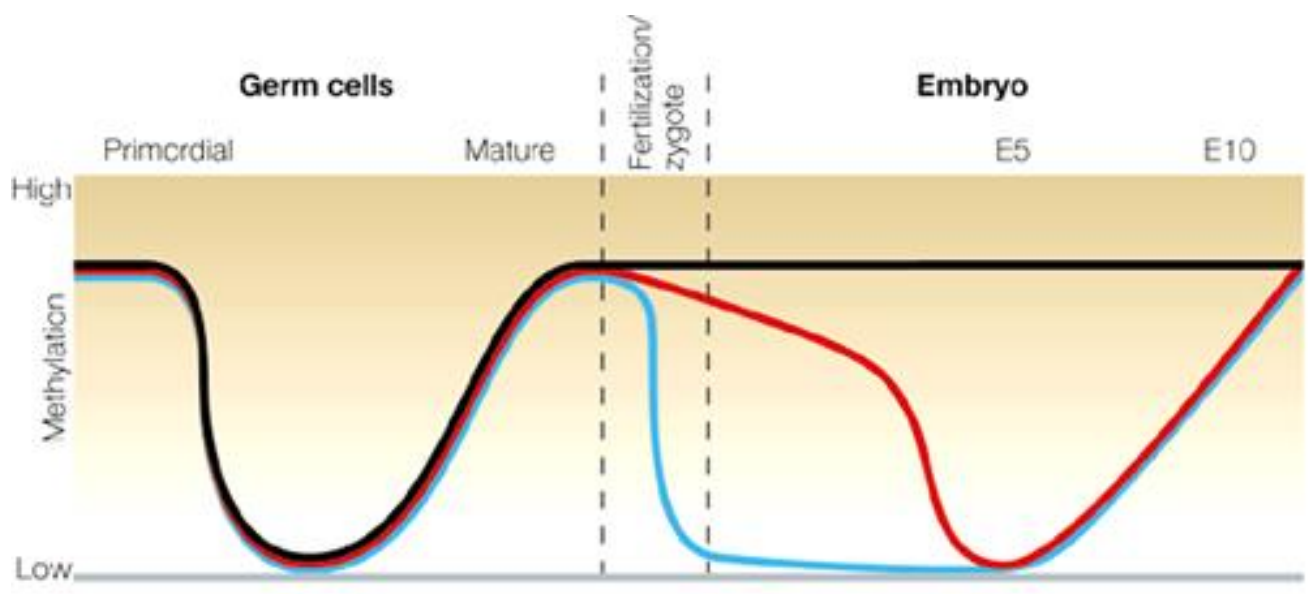

Nature Reviews | Genetics

Figure 6.7: Development of global methylation status in mouse germ cells and early embryos. 
The changes in chromatin structure of the early embryo play an important role in genome activation but alone are not sufficient to activate transcription. The best proof is a nuclear transfer experiment because the activity of transcriptionally competent nuclei to support transcription depends on the developmental stage of the recipient cytoplasm. The changes in cytoplasm, especially availability, content, or activity of transcription factors, play also important role in genome activation.

\subsubsection{Regulation of EGA via transcription}

EGA entails a dramatic reprogramming of gene expression that must be faithfully executed in order for development to proceed. Whereas EGA was previously thought to be relatively promiscuous due to the extensive chromatin remodeling, results of microarray experiments demonstrate that genes involved in transcription, RNA processing, and cell cycle regulation are preferentially expressed.

Since embryonic transcription initially depends on the time elapsed after oocyte activation, it has been proposed that a "zygotic clock" controls the processing of maternally inherited factors to convert sperm and oocyte chromatin into transcriptionally competent matrices accessible to transactivating factors (4). The molecular nature of this clock is not known but it is proposed to regulate proteinprotein interactions between three components required for transcriptional activation in vivo (Kaneko, K.J., DePamphilis, M.L., 1998):

$>$ activators bound to enhancers sequences

$>$ protein complexes such as the TATA-binding protein (TBP), and associated transcription activating factors (TAF)

$>$ functional assembly of the RNA polymerase holoenzyme bound to the site of transcription initiation.

Not only different components but also different mechanisms are implicated in the silencing and, thus, in regulation of the embryonic genome during early development and control zygotic clock (Schier, A., 2007):

\section{$>$ chromatin-mediated repression}

Previous studies suggested that the nucleo-cytoplasmic ratio and the titration of a transcriptional repressor by the exponentially increasing amount of genomic DNA determine the timing of EGA (excess repressor model). However, the key factors that need to be titrated are still elusive, but it is conceivable that histones or other chromatin components maintain silencing until a critical amount of DNA is present. Gene-specific DNA methylation has also been implicated in repression. Depletion of the methyltransferase Dnmt 1 or of Kaiso protein, a transcriptional repressor binding to methylated 
DNA, results in the premature activation of a subset of genes. These evidences suggest that multiple chromatin-mediated mechanisms prevent EGA.

deficiencies in the transcription machinery

A dearth or inactivation of components of the transcription machinery might be a second strategy for gene silencing before EGA (limited machinery model). For example, premature expression of TATAbinding protein, a component of the transcriptional machinery, can induce premature activation of a subset of genes. Analogously, mis-expression of strong transcriptional activators can cause precocious transciptional activation.

transcriptional repression or abortion by rapid cell cycle

The rapid cell cycles lacking G1 and G2 phases might constitute a third strategy that interferes with productive transcription during early cleavage stages (rapid cell cycle model). DNA replication can interfere with transcription, and mitosis can lead to the abortion of nascent transcripts. Conversely, experimentally induced cell cycle lengthening can lead to premature transcription.

Although these silencing models can account for some aspects of EGA, they do not explain why several genes are already activated during early cleavage stages and why zygotic genome activation is a gradual and gene-specific process. It is conceivable that in mammals there might be sufficient time to assemble transcription complexes at early cleavage stages because the cell cycles are very long.

\subsubsection{Enhancers}

Enhancers, which are located more distal to the transcription start site than promoters, are proposed to have two functions, namely to recruit RNA polymerase to the promoter and/or to relieve chromatin-mediated repression of a weak promoter. Enhancers are required for efficient transcription following genome activation and that the function of these enhancers is to relieve chromatin-mediated repression that develops concomitant with genome activation.

The developmental acquisition of an enhancer requirement for efficient gene expression requires the appearance of a co-activator. Enhancers are inactive in oocytes and embryos before EGA, even when the appropriate sequence-specific activation protein is present, because an enhancer-specific co-activator activity is not produced until EGA (Schultz, R.M., 2002). This suggests that one or more transcription factors are produced at this time whose function is to activate genes required for preimplantation development via activation of their enhancers. One of the proved regulating 
enhancer's co-activator is TEAD-2 protein (Kaneko, K.J., DePamphilis, M.L., 1998), which binds to the same DNA sequence as the transcription enhancer factor (TEF)-1. Only TEAD-2 is significantly expressed in the preimplantation embryo, and its expression is derived from maternal mRNAs that are recruited during the EGA. The interactions between maternally derived components and de novo created genome are also an evidence of tight co-operation required for successful EGA.

\subsubsection{Promoters}

Promoter is a region of DNA to which RNA polymerase binds before initiating the transcription of DNA into RNA. Most transcription factors regulate gene transcription do so by binding at or near the promoter and affecting the initiation of transcription.

Initiation factor eIF-1A displays a transient increase in expression during EGA. The eIF-1A contains both a proximal TATA-containing promoter and a distal TATA-less promoter. It was noted that while $70 \%$ of the transcripts present in the oocyte are derived from the TATA-containing promoter, by the 2- to 8-16 cell stages only 10-25\% result from transcription initiated from the TATA-containing promoter. The change in TATA box utilization may be a general property of genome activation such that TATA-less promoters are more efficiently used.

There are two obvious biological consequences of such a switch in promoter utilization, the first being that many housekeeping genes are regulated by a TATA-less promoter. Preimplantation development is accompanied by a dramatic increase in metabolism. The more efficient use of TATAless promoters that accompanies development would provide a convenient mechanism to foster an increased expression of housekeeping genes that in turn could support this increased energy demand. Such a change could also contribute significantly to the reprogramming of gene expression that occurs during EGA.

Another consequence of this switch in promoter utilization would be to enhance the expression of genes critical to preimplantation development. For example, oct-4, which is required for the maintenance of totipotent blastomeres of the inner cell mass, is driven by a TATA-less promoter. The more efficient use of TATA-less promoters that accompanies genome activation could ensure that sufficient levels of oct-4 expression are maintained during preimplantation development.

\subsubsection{Transcription machinery}

A functional RNA polymerase II is already present in 1-cell stage in mouse embryos and can gain access to promoters in the absence of DNA replication. Since other transcription factors are also detectable in pronuclei, transcription may be initiated rapidly after fertilization. However, the large 
protein complex that constitutes the transcriptional machinery can also directly influence all steps in transcription, from initiation to termination.

Phosphorylation of the carboxyl-terminal domain (CTD) of the largest RNA polymerase II subunit is critical in regulating the initiation of transcription (Dahmus, M.E., 1996). The CTD contributes to interaction of the polymerase with DNA, transcription activators, TBP, and, has recently been implicated in the RNA processing events that accompany transcription (McCracken, S. et al., 1997). Moreover, as the polymerase undergoes the cycle of initiation, elongation and termination of transcription before reinitiating or de novo initiating of a new round of transcription, the CTD undergoes a parallel cycle of phosphorylation and dephosphorylation.

The CTD, which is predominantly hyperphosphorylated in transcriptionally inactive unfertilized oocytes, is markedly dephosphorylated 2-3 hrs after pronuclei become visible, but before the onset of minor genomic activation. The minor activation then occurs with re-phosphorylation of the large RNA polymerase II subunit but not to the same state as that normally observed in somatic cells. The presence of this embryo-specific phosphorylated form persists to major genome activation.

A great number of transcription factors are expressed as maternally derived proteins in the early embryo. Transcript abundance of factors Sp1, TBP, CBP and mTEAD-2 starts to decrease during oocyte maturation and then increases at the 2-cell stage. This pattern reflects the degradation of maternal mRNA that is initiated during oocyte maturation and the replacement of these maternal transcripts with zygotic ones following activation of the embryonic genome.

\subsubsection{Others}

In dissecting the molecules and molecular mechanisms that control mammalian oocyte-toembryo transition, scientists have lately discovered a possible role of developmentally regulated endogenous retroviruses (ERV) in mouse oocyte and preimplantation embryos. These can act as alternative promoters and first exons for diverse genes, synchronizing their expression. ERV are usually epigenetically silenced, through DNA methylation and chromatin-based mechanisms. Their activation and silencing indicated a change in the epigenetic state of the genome. The thousands of endogenous retro-elements in the mouse genome (and other mammalian) provides potential scope for large-scale co-ordinated epigenetic fluctuations and leads to the hypothesis that differential transposable element expression triggers sequential reprogramming of the embryonic genome during the EGA. 
Abbreviations:

A - adenin

C - cytosine

CTD - carboxyl-terminal domain

DFC - dense fibrillar component

DNA - deoxyribonucleic acid

Dnmt - DNA methyl transferase

eIF-1A - eukaryotic initiation factor $1 \mathrm{~A}$

EGA - embryonic genome activation

ER - endoplasmatic reticulum

ERV - endogenous retrovirus

FC - fibrillar center

$\mathrm{G}$ - guanin

GC - granular component

$\mathrm{H}$ - histone

IAP - intracisternal-A-partical

MII - metaphase II

MET - maternal embryonic transition

mRNA - mediator (messenger) ribonucleic acid

NPB - nucleolus precursor body

PGC - primordial germ cell

RNA - ribonucleic acid

$\mathrm{T}$ - thimidin

TAF - transcription activating factor

TBP - TATA box-binding protein

TEAD - TEA domain family transcription factor

TEF - transcription enhancer factor

ZGA - zygotic genome activation 


\section{Micromanipulation in mammalian embryology}

\subsection{Introduction}

Micromanipulators are instruments designed to move small tools (for example capillaries, knives and needles) under a microscope to perform delicate procedures. The most common application of micromanipulators is the field of mammalian embryology, where these instruments are used to perform microsurgical interventions in gametes and embryos. Micromanipulation in strictest sense means the application of micromanipulators. However, this definition would unreasonably restrict the subject of this chapter. In a broader sense a kind of micromanipulation may also be performed with skilled hands and mouth pipettes, as it has happened before the introduction of micromanipulators, and as it happens recently when simplification of delicate procedures (performed previously exclusively with micromanipulators) allow the use of handhold tools with the same (or even higher) efficiency. Additionally, the use and importance of micromanipulation can only be explained if we also describe the purpose and other definitive steps of the given procedure.

\subsubsection{History}

Micromanipulation in mammalian reproductive biology may not require the use of micromanipulators, but two other factors are indispensable: 1 . appropriate microscopes, and 2. basic knowledge about the requirements to maintain structural integrity and functional competence of mammalian oocytes and embryos for full-term development.

Although matured (MII phase) oocytes are the largest cells of the mammalian body, even with their diameter (around or slightly more than $0.1 \mathrm{~mm}$, depending on species) they are almost invisible by naked eye, and a common magnifying glass is still inappropriate to provide the required 30- to 100- (for some purposes up to 300-)-fold magnification form micromanipulation. Quite surprisingly, that magnification has already been achieved as early as in the turn of the 18th century in the Netherlands, a new country with rapidly growing navy and commercial fleet, and with the need for delicate lenses for telescopes. The inventor (and the founder of microscopic investigation in biology) was not a scientist but a merchant called Antonie Philips van Leeuwenhoek, and for long his observations were disregarded by the scientific community. However, eventually the discoveries regarding cells, spermatozoa, or even bacteria were acknowledged, with the highest possible acknowledgement, by attempts to steal the priority of the discovery.

One would suppose that one hundred years after the discovery of the compound telescopes (with multiply lenses) the first really efficient microscope of the world would also work on the same principle. Surprisingly, although compound microscopes existed already in the time of Leeuwenhoek, 
they were extremely large and produced very low (15 to 20 fold magnification) and poor visibility. On the other hand, the single tiny lens in the middle of Leeuwenhoek's microscope (the full size of which was close to a stamp and was hold close to the observer's eye like a monocle) has produced up to 300 fold magnification making all these biological observations possible. Unfortunately, this great achievement has meant at the same time a dead end, no further improvement upon these principles was possible, and the structure did not allow any manipulation of the observed cells, either. Eventually the sequential improvements of the compound microscope with objectives, oculars, then appropriate illumination system and stages have resulted in the appropriate optical control for micromanipulation. It was an Englishman, peer of Leeuwenhoek, called Robert Hooke, who has made the initial steps of this process. Although the principles did not change much during these centuries, the quality of lenses and consequently the quality of view has radically improved even during the past 25 years.

Although the improvement is continuous, the basic optical background for micromanipulation has already been established before the end of the 19th century, and a handful of scientist have already started to make manipulations on embryos. Their initial purpose was to answer the basic question of developmental biology: is the differentiation a reversible or irreversible process? Does the 1st, 2nd or 25th cleavage of mammalian cells induce irreversible changes or are both cells of a two-cell embryo, or terminally differentiated cells of an adult organism capable to govern the development from the very beginning?

The question has been answered fully only in 1997, with the birth of the first somatic cell cloned animal, Dolly. However, regarding the first cell cycle stages, early experiments performed with sea urchins and salamander did provide the exact answer to the initial question. The separation of blastomeres resulted in healthy twin offspring. The initial work was performed by simple shaking of sea urchin embryos, therefore cannot be considered as micromanipulation. However, shortly afterwards, in 1914, a German scientist, Hans Spemann has performed a real micromanipulation in salamanders by hand, using a loop produced from the fine hair of his baby son. This famous experiment may be regarded as the ancestor of all further micromanipulations, and resulted in a strange by-product, as well. When the strangulation was unsuccessful, and only partially separated a cytoplasmic part without nucleus, this part failed to develop further. However, if the loop was slightly released and a nucleus migrated into this extrusion, this part has started to develop again, could be separated (by fastening the loop) from the other parts, and has developed to a healthy animal.

Spemann was an extremely creative and clever biologist. His work in other fields of developmental biology was honoured with a Nobel prize. However, this observation with the salamanders was almost forgotten by everybody, except for himself. Decades later, in his book 
(published in 1938) he designed a "fantastic experiment" on the basis of the previous observation: to remove the nucleus from an oocyte, and replace it with a nucleus of a somatic cell. He added, however, that he does not see a realistic way to perform any of these steps.

The two American scientists, Robert Briggs and Thomas King, who eventually performed this fantastic experiment, did not know anything about Spemann's suggestion. Their project was evaluated as harebrained and the requested financial support was refused by the National Cancer Institute. Finally, after a repeated application they managed to convince the committee about some slight chances of success. The slight chance has become a reality. Many factors contributed in the success, including the use of eggs of a common frog. The cheap animal and the large number of produced eggs provided virtually unlimited quantities for experimentation (a dream for a present day human embryologist), and the large size of the egg enabled them to aspirate the nucleus and to replace it with another one from a somatic cell, that was injected into the oocyte. After hundreds and hundreds of fiascos, eventually some of the reconstructed embryos developed to blastocysts.

These initial experiments were performed by hand, but subsequently they have started to use some primitive micromanipulators for the purpose. These were the first documented trials when micromanipulators were successfully used in embryology, and resulted in eventually the established classic model of nuclear transfer cloning. However, it should be noted, that in spite of the tens of thousands of experiments performed by the two Americans and their successors, nobody could achieve a full success. Somatic cells from tadpoles supported full term development of enucleated oocytes to adult frogs; somatic cells from adult frogs produced tadpoles, but nobody could produce adult frogs from somatic cells derived from adult frogs. The explanation is still missing, but in spite of the great achievement, frogs failed to provide the final evidence for totipotency of an adult somatic cell.

And, these are only amphibians. No similar achievements were published, or even outlined in mammalian embryology. Unfortunately, apart from some preliminary attempts, we could not talk about mammalian embryology that time, in the middle of the 19th century, after the explosions of the first $\mathrm{A}$ and $\mathrm{H}$ bombs, shortly before the launch of the Sputnik.

Retrospectively, it sounds nonsense, but a capital mistake has hampered the development of mammalian embryos in vitro. Scientist did not know that both oocytes and embryos are extremely sensitive to temperature. Many other cells, or whole organs including transplanted human hearts, kidneys or livers survive ambient temperatures or refrigeration for hours. However, when mammalian oocytes are removed from the follicles, they become extremely sensitive to any thermal effect. Exposition to room temperature for minutes, or longer in vitro culture on $0.5^{\circ} \mathrm{C}$ higher or lower than the core temperature of the given species may damage irreversibly mammalian oocytes and 
preimplantation stage embryos. The failure to realize this simple fact has hampered the development of mammalian embryos for at least 60 years, from the late 19th century till the middle of the 20th century.

Compared to this delay, the achievement during the past 50 years is rather impressing. However, embryology still has remained a handicapped branch of science. In contrast to the common opinion, domestic animal and human embryologists definitely know that the instrumental basis of this technology is very far from "high technology", may rather be called as "low technology". Researchers try desperately to make 21 st century science with 19 th century tools and approaches. Mammalian embryology is a very special and narrow area, disregarded by most commercial companies. The market is too small to produce special tools, or to perform changes on the existing products, according to the special needs. A molecular biologist of the year 1987 would not find anything resembling his late tools in a modern laboratory; however, almost nothing has changed in an embryology laboratory during the past 20 , or even 30 years. In the age of the gigabytes and nanorobots, it is definitely a shame that there is no real alternative of mouth-pipetting for delicate practical handling of oocytes and embryos.

The only area where considerable advancement was achieved was the micromanipulation and related tools. The first micromanipulators used for mammalian embryos were primitive tools and required homemade adjustments, including the experiments of Andrzej Krzysztof Tarkowski, who has used the Warszawa telephone book to support his micromanipulators. Even so, his pioneering work in chimaera production (i.e. merging two or more embryos to produce a single offspring of mixed origin) opened new perspectives for basic developmental biology of early embryo development.

The subsequent, and probably most important breakthrough in the field of micromanipulation was performed by a Danish scientist in England. Steen Malte Willadsen, has started his career as a country veterinarian in a remote part of Denmark, but his ambitions forced him to return to the university as a researcher, and eventually to accept a position in Cambridge. Grants those times were not more generous than today, and Willadsen had less than minimal support. However, he could not be stopped. He had some visions, and in a few years he realized every single one. While working almost entirely alone with a flock of sheep, being the shepherd as well as the veterinarian, in his spare time he re-created the principles and tools of micromanipulation and opened entirely new fields for mammalian embryology. He has halved and merged embryos, embedded them in agar chips, transferred them to the uterus of temporary recipients, and created offspring with recovery of these embryos and transfer into new surrogate animals. He discovered the way how to make appropriate microtools (capillaries, knives, holders), and how to use them with inimitable efficiency. He has made 
interspecies chimaerae, mixtures of cow and sheep, sheep and goat. He introduced electrofusion as the most efficient and elegant way to merge oocytes and embryos with each other or with other cells. Finally, he established mammalian cloning by nuclear transfer with exactly the same technology that was used a decade later to create Dolly. The only difference between his work and the famous experiment was that in Willadsen's experiments the donor cells were derived from an early embryo.

With Willadsen, the history of micromanipulation, the era of pioneers has ended. The past twenty years from technical point of view was the period of small steps, refinements, standardizations and diverse applications, without significant changes in principles..

\subsection{The principles of micromanipulation}

All micromanipulator are based on the principle that they decrease the dimensions of the movements of our hands with at least one order of magnitude. For example 1 centimetre movement of our fingers (moving in most micromanipulators a joystick) may result in 1 millimetre or less movement of the tools, precisely in the required direction in all the three dimensions, and precisely according to the predetermined proportion. This proportional decrease can be achieved with either simple or sophisticated mechanical structures, hydraulic or electronic regulation. Although there are enormous differences in prices between micromanipulators, a well-built and maintained mechanical tool may be almost as appropriate in skilled hands than the ten- or twenty-fold more expensive attractive instrument with microprocessor-regulated electronic motors. The arms of the micromanipulators a metal tube, commonly called micropipette holder, and the various tools (mainly glass capillaries or glass needles) are attached to this tube. A typical micromanipulator set consist of two micromanipulators (left and right), and one or (rarely) two tubes are attached to their arms. Traditionally (and according to the right handed majority of researchers) the left micromanipulator is used to hold the embryo, while the right one is to perform various interventions. For some purposes, for example embryo bisection in cattle, some companies offer only one micromanipulator, and suggest to use protein-free media to fix transiently the embryos to the bottom of the plastic dish. However, this application does not allow oriented bisection; moreover, the whole work can be performed without any micromanipulators, with higher efficiency and speed (see discussed later).

Most micromanipulators are also equipped with special syringes to produce delicate negative or positive pressures in capillaries for aspiration or expulsion of extremely small and precisely controllable amounts of solutions into or from the glas capillaries. The connection between the syringes and glass capillaries is established with plastic tubes with rigid walls that are resistant to expansion and forward exactly the same amount of solution to the capillaries that is injected into them by the syringes. The system is filled usually by oil without any air bubbles, to avoid the buffering 
effect of the gas. It should be mentioned, however, that some experts of micromanipulation do not use oil as transmitter, but fill the system entirely with air, and use it with surprisingly good efficiency.

Capillaries attached to the metal tubes are constructed according to the special needs. One end of them is wider and has a thick wall, this part is attached to the metal and plastic tubes. The other end is narrow, and finely shaped. The holder capillary is relatively wide, and the end of it is polished and considerably narrowed with a flame. The outer diameter is approximately identical with the diameter of the oocyte and embryo, while the inner diameter of the opening is between 10 to $20 \%$ of the outer diameter. According to this shape, a delicate suction fixes safely the zona pellucida of the oocyte or embryo, causing a minimal and completely reversible misshape on the zona, while the shape of the embryo remains intact.

In contrast to the mainly uniform holding pipettes, microtools used to perform the manipulation differ considerably according to the purpose, species, developmental stage and the method used. Microneedles are extremely narrow glass needles formed from capillaries, but without any opening at the end. The typical purpose of the work with these needles is to make a linear opening on the zona pellucida by passing through the zona at two points, i.e. in and out, without touching the embryo inside; then releasing the embryo from the holding pipette, and rub it to the side of the holding pipette until a linear slit occurs. This slit may help the embryo to hatch (mechanical assisted hatching), but may also be used to enucleate oocytes with subsequent mechanical pressure on both sides of the oocytes until the appropriate part of the cytoplasm containing the chromosomes extrudes. A release of the pressure results in a virtual closure of the zona and separation of the nuclear material outside from most of the cytoplasm that has remained inside the zona. A similar application is one possible way of blastocyst biopsy: during initial expansion, a small part of the embryo is extruded through the slit, and can be separated from the rest of the embryo with pipetting.

Various injecting or aspirating capillaries exist that are produced to pass through the zona pellucida, and remove some part of the oocytes or embryo, or inject a cell in the perivitelline space or the embryo itself. The shape of these capillaries resembles the end of a common injection needle. The size may be completely different according to the special purpose. Capillaries that are used for blastomere biopsy are relatively large, while others used to transfer the sperm into the cytoplasm of the oocytes at intracytoplasmic sperm injection are among the smallest, according to the size of spermatozoa.

These basic tools are only for the most common applications. Different shapes are needed for chemically induced assisted hatching, and some embryologists use needles and blunt end capillaries for blastomere biopsy, first to make a slit, then enter and aspirate the blastomere with a fire-polished capillary to avoid any damage. Other applications may require special forms of needles or capillaries. 
Although some earlier manipulations were performed in a specially constructed sample-holding chamber that enabled work with completely straight glass capillaries, most recent manipulations are performed in Petri dishes, requiring formation of a (typically $30^{\circ}$ ) angle close to the narrow end of the capillary, enabling the tool to get immersed into the solution while the working end remains horizontal.

Five years ago, in another book dealing with embryology I wrote: "No professional micromanipulator embryologist would ever use ready-made glass microtools. It is a matter of prestige to create these tools for him- or herself from simple straight, thick capillaries, with specially developed, sophisticated and expensive laboratory instruments including capillary pullers, glass grinders and microforges." The proper use of these instruments was an art itself, and embryologists handled their secrets - the bases of their successful work - with the greatest confidence.

I have to acknowledge now that these days are over. As micromanipulation has become an indispensable tool of human assisted reproduction (probably the only area of embryology where financial support is more or less appropriate) companies discovered the emerging market and started to produce ready-to-use glass tools, with versatility, accuracy and consistency that is simply over the possibilities of the homemade manufacturing. Moreover, with the large scale production prices have dropped to a level that is also affordable to a research laboratory or a commercial unit working on animal field. Accordingly, the toolmaking instruments are not standard part of a micromanipulation laboratory any more. They are rather curiosities of the past and used only occassionally for preparing some very special microtool.

Per definitionem, micromanipulators are connected to a microscope. For simple procedures stereomicroscopes are appropriate, especially with the latest improvements in the optical and mechanical structure that has almost doubled the achievable (or rather the practically usable) magnification. The unique benefit of application of stereomicroscopes in micromanipulation is the simplicity, they can be regarded as a fixed magnifying glass. The access to the specimen and microtools is easy and evident, consequently the use does not require much learning, can be acquired in several days or a week. even by laymen not familiar in embryo handling techniques. The disadvantage, however, is that even with the abovementioned improvements, stereomicroscopes offer maximum 20 or $30 \%$ of the magnification of a traditional microscope, where the objective is positioned extremely close to the specimen (at high magnification, the distance is around or less than $1 \mathrm{~mm}$ ). Accordingly, when accuracy is a critical factor, or the view of some subcellular structures is indispensable, stereomicroscopes are insufficient. Moreover, most of the latest sophisticated optical modifications (see discussed later) that help to identify these structures cannot be applied for stereomicroscopes. 
Most micromanipulators are built on an inverted microscope. The name "inverted" refers an upside-down arrangement, where the objective reaches the specimen from beneath. This situation offers two major benefits: when observing specimens that are hold in solutions (i.e. all materials in embryology), the objective should not be immersed into the solution with all consequences (contamination, practical handling, etc). The other benefit is that over the level of the solution, above the specimen there is an appropriate space to insert and handle all the micromanipulaton tools.

There are many different types of inverted microscopes. Those commonly used for micromanipulation of oocytes and embryos are rather sophisticated and expensive ones. They offer uniformly sharp view and wide angle even at high magnification. The stage holding the specimen is relatively large and the majority of the area is constructed from glass enabling work on the various points of a $60 \mathrm{~mm}$ diameter Petri dish commonly used for micromanipulation. On the other hand, the stage including - or rather predominantly - the glass part should be evenly heated precisely to the required temperature, i.e. the core temperature of the mammal the oocytes and embryos are originated from. As micromanipulation may be a long procedure, the lack of appropriate heating may be detrimental. Some inverted microscopes are even equipped with a large transparent plastic box enclosing the whole working area, thus providing very stabile temperature and (if required) gas atmosphere with the required composition.

During the past decades, a considerable advancement in the optical systems has also been achieved. Apart from the common transmitted light, phase-contrast and UV illuminations, new systems have been developed, some of them directly for embryological purposes. These systems include Nomarski differential inteference contrast (DIC) optics (for glass dishes) and Hoffman modulation contrast optics (for plastic dishes), and very lately a special polarized light microscope called Oosight. With the use of these optical systems a better clarity and surprisingly informative view - in some instances three-dimensional-like images - are available, while other tools offer perfect view of condensed chromosomes in living oocytes of some species including primates, enabling oriented enucleation in Metaphase II stage during nuclear transfer.

Latest advancements of micromanipulations include the use of some very special mechanisms for certain application. A laser beam can be directed to certain structures (most frequently to a segment of the zona pellucida) to open a small hole by destroying the structure while leaving the cells inside undamaged. Piezo micromanipulation enables embryologist to relatively smooth passage through cell membranes, for example for injection of cells into oocytes. These new tools certainly help to perform some procedures. However, their application is still limited due to the high price of these instruments. It should also be noted that the majority (although not all) of the procedures where laser and piezo micromanipulators are used can also be performed with more primitive interventions 
(for example a directed stream of acidic Tyrode solution may open a similar hole on the zona pellucida of the human oocytes).

Microscopes and micromanipulators are usually placed tp the farthest end of the embryo laboratory, providing a relatively calm environment for the work. However, research institutes and hospital laboratories are typically located in multistore buildings with considerable RESONANCE. On the other hand, common laboratory benches do not offer enough stability for these heavy but very sensitive instruments, especially when other heavy instruments, mixers, shakers and centrifuges are operated in the neighbouring laboratories. An initial solution for the problem was to buy extremely heavy and stabile desks composed of marble or other stones. Later, special benches with inserted resonance-free parts were produced, similar to those offered for laboratory balances. Eventually the problem could also be resolved by placing the microscope and micromanipulators on a heavy metal sheet that is supported by numerous soft foam globes of the size of a tennis ball. This simple solution may offer surprisingly good results. However, it should be also noted that in most situation the resonance problem is overestimated, and serves as an apology for the less skilled embryologists.

\subsection{Application of micromanipulators in mammalian embryology}

Although some applications have already been mentioned previously, this chapter will shortly summarize the most important technologies and purposes.

\subsubsection{Embryo bisection}

This is one of the simplest techniques of micromanipulation. It is applied exclusively in animal embryology, mostly in domestic animals, i.e. in cattle. The purpose is to make identical twins from a single embryo with valuable genetic traits. In the morula stage, the position of the embryo is irrelevant, therefore the bisection can be performed without a holding pipette. As in the absence of proteins the zona pellucida attaches reversibly to the bottom of the dish, this simple change of medium is enough to fix the embryos temporarily and perform the bisection with a metal microblade, under the control of a stereomicroscope. However, when the work is performed on blastocysts, proper positioning (and the use of holding pipette) is required, as the inner cell mass should also be bisected. Subsequently the two halves can be cryopreserved or transferred to the recipient animal. In contrast to the earlier expectations this technique results in only a moderate increase (if any) in live offspring rates, most probably due to the damage during manipulation and the decreased cell number of the halves. Another bisection, i.e. to form quarters instead of halves results in even more compromised cumulative outcome. Accordingly the application fields of the technique are rather limited. 


\subsubsection{Embryo biopsy}

In contrast to embryo halving, embryo biopsy is a technique with rapidly increasing application both in animal and human embryology. The purpose of the work is to remove one cell or a small group of cells from the embryo and use these cells for diagnostic purposes. A single cell (or maximum two cells) can be removed from an eight-cell stage human or bovine embryo before compaction. Various methods can be used for this procedure. As described earlier, a hole can be made on the zona pellucida with acidic Tyrode or laser beam (the technique is called zona drilling), and the blastomere can be removed with a fire-polished capillary. However, the procedure may also be performed with a large injection pipette by using it at the same time to pass through the zona and to remove the cells. The disadvantage of the latter procedure is that it may result in more mechanical trauma to the embryo. For blastocyst biopsy, the simplest way is the previously mentioned slot created with a microneedle, and removal of the subsequent extrusion of the trophectodermal cells. However, biopsy for certain purposes can also be done on oocytes before or after the fertilization, by removing the first or second polar body.

At present, the samples are almost exclusively analysed for their DNA content. This enables us to determine the sex of the embryo, the presence of chromosomal abnormalities and certain genetic disorders. The possibilities for analysis are increasing exponentially, and in the foreseeable future the whole genome analysis seems to be also an achievable goal. The purpose of the procedures is to avoid transfer of embryos with inherited or acquired genetic abnormalities in human fields, and to transfer embryos with the most advantageous genetic composition in animal breeding. While the latter purpose is to be realized, the diagnostic procedure in human has become a routine in most assisted reproduction laboratories. The application areas are virtually unlimited, and may also raise some ethical questions including the controversial use of the technique to determine the gender of the child according to the wishes of the parents.

A procedure technically resembling biopsy but with no diagnostic purposes is used in some situations to improve embryo quality. Cryopreservation of human embryos may result in death of some blastomeres: according to several publication, the removal of these cells may improve implantation and full-term developmental rates. On the other hand, the extreme amount of lipid droplets in a normal porcine embryo hampers any cryopreservation due to the irreversible chilling damage that occurs in these embryos during cooling and warming. High speed centrifugation may polarize these lipid droplets, and their subsequent removal by aspiration improves the chances of survival of the cryopreservation procedure. 


\subsubsection{Subzonal and intracytoplasmic sperm injection}

A common and increasing factor in human infertility is the inappropriate quality or quantity of human sperm. In some cases in vitro fertilization or analogue procedures may requiring lower sperm numbers than natural fertilization may offer a solution for the problem. However, in the majority of male infertility problems even these assisted reproductive techniques are insufficient to achieve fertilization. Fertilization, i.e. passage through the zona pellucida followed by penetration in the ooplasm the oolemma is a delicate procedure. Under natural circumstances it requires a morphologically normal, fully matured and motile spermatozoon with the appropriate (capacitated) functional stage. Curiously, for the subsequent steps these conditions are not indispensable. Once the sperm gets actively or passively into the ooplasm, motility and capacitated stage are not required for the further steps; moreover, successful fertilization can also be achieved with immature stages, with round spermatids obtained by biopsy form the testicles (although the latter procedure is still regarded by many human embryologists as experimental).

Initially, spermatozoa were just injected in the perivitelline space, under the zona pellucida, and aside the oocyte with a technique called Subzonal Injection (SUZI) with limited success. Although the Singaporean scientist, Soon-Chye NG, who first applied SUZI has also described an alternative technique, Micro-Insemination Micro-Injection into Cytoplasm (MIMIC), but it was entirely disregarded it by embryologists for two years, when Palermo in Belgium described the same technique with the name of Intracytoplasmic Sperm Injection (ICSI). During the subsequent decade intracytoplasmic injection has soon replaced entirely subzonal injection, and at present this is the most frequently applied fertilization technique in assisted human reproduction. In an average human laboratory 60 to $90 \%$ of the embryos are produced by ICSI. The earlier concerns regarding the increased number of developmental abnormalities following ICSI haven't been unanimously justified. Another concern regarding the "reproduction of reproductive problems" may only be evaluated in 15 to 25 years, when large numbers of men fertilized with ICSI enter the fertile age. However, this strange situation is more or less applicable to all fertility problems. A discrimination based on a potential genetic handicap, refusal of the right of couples to have babies on the basis that the next generation would face even more infertility problems is unacceptable and may be regarded as a kind of eugenics. On the other hand, and this is also a factor to be considered, human fertility clinics do not stop making procedures just because the procedure in long term will result for them even more patients.

From technical point of view, ICSI is probably the most demanding micromanipulation procedure. As the result can be evaluated in 24 hours, with exact percentages, and the product is a 
highly valuable human embryo, there is little space for excuses. It must be performed with the highest possible efficiency.

The first step of the procedure is to immobilize the spermatozoa by damaging the plasmalemma. This immobilization may prevent disruption of the ooplasm, and may promote oocyte activation. It is usually performed with the narrow, injection needle-shaped injection pipette by pressing spermatozoa to te bottom of the dish, and/or with a rapid movement of the pipette from left to right to ensure plasmalemma damage. PVP is commonly applied to slow down the movement of the spermatozoa, to ensure that the supposedly best ones with high motility could be caught.

Following immobilization, spermatozoa are aspirated into the injection pipette and injected into the cytoplasm of the oocytes. This second step is also demanding, as the pipette has to pass through the zona and subsequently the oolemma,, without making lethal damage. Then a large cylinder of ooplasm is aspirated into the injection pipette, and finally, with a slow movement, this cylinder is expelled back - together with the sperm cell - into the oocyte. If performed appropriately, the sperm should remain in the ooplasm, and upon retraction of the pipette the oolemma should close immediately. Further handling of oocytes does not differ of that after in vitro fertilization.

Paradoxically, ICSI is quite inefficient in domestic animals, and the application fields are also very limited.

\subsubsection{DNA microinjection}

The first genetically modified mammals, mice were produced by microinjection of the foreign DNA into the pronuclei of zygotes. In a relative high percentage the DNA is integrated into the genome, and although the integration is random, it is frequently stabile. If no other, important genes are damaged during the integration, the foreign gene will be present in all cells of the offspring including germ cells, accordingly it will be inherited to the next generations. The expression of the gene may be general or either tissue or developmental stage-specific according to the injected construct. The injection is performed quite similarly to the sperm injection, but the sample should be precisely delivered into one of the two pronuclei. Its swelling is the indicator of the successful targeting and also the measure of the appropriate quantity.

Unfortunately this technique can only be successfully applied in animals where the cytoplasm of the oocytes is transparent allowing clear visibility. In most domestic species including cattle and pig, the high amount of lipid accumulated in the cytoplasm hampers identification of pronuclei. Although lipids may be removed with centrifugation and subsequent micromanipulaton (see discussed earlier), the cumulative effect of the procedures is a rather disappointing efficiency. The lack of possibility to perform directed changes (exact integration to the required site, enabling either precisely modified or 
deleted functions) limits also the applications in mice, where other, more sophisticated and precise technologies are also available for transgenic animal production.

\subsubsection{Chimaera formation}

Chimaera is a Latinised Greek word, and as usual, there is a disagreement in the correct English spelling (chimaera vs. chimera). The generally accepted English plural is by all means incorrect instead of chimaeras or chimeras, the correct and consequent use of Latin terms would require chimaerae - as for morulae.

Moreover, originally Chimaera was a once-even creature. She had a definite gender, as the word in Greek (Xı $\mu \alpha 1 \rho \alpha$ - Khimaira) means she-goat. She was an animal that had the body of a goat, the head of a lion and the tail of a serpent (some imagine Chimaera as a three-headed creature - one of her surnames is trikephalos -, but the myths speak about a single head which was vomiting or breathing fire). She was extremely malignant. She has devastated the country, and finally was killed by Belleraphone while he was on the winged horse of Pegasus.

Accordingly, the choice of word is not quite fortunate, but widely accepted today. The biological definition of chimaera is the following: an animal (or plant) made up from a mixture of cells from more than one species. Also (in human genetics), an individual made up from cells derived from two or more zygotes. Other mixed creatures include hybrids referring an organism made by crossing two different species (with normal fertilization) and mosaics where all cells have the same embryonic origin but a radom population of cells is genetically different from the rest. Although not known widely, chimaeras may occur naturally, even in humans. Non-identical twin sister embryos may fuse in the womb and grow into a single body.

Production of mammalian chimaeras was first reported in mouse by Tarkowski (1961; with the spelling: chimæra) and Mintz (1962). Later, live chimaeric offspring have been produced later in sheep, cattle, pigs and goats. Chimaeric offspring were produced between closely related species, including Bos taurus and Bos indicus, goat and sheep, and between distant relatives as cattle and sheep.

Methods used for the production of animal chimaeras include aggregation of blastomeres from early cleavage stage embryos or morulae inside the zona pellucida, injection of various cell types into the blastocyst cavity, and complete inner cell mass transplantation based on immunosurgical isolation. All these methods require micromanipulators, are technically demanding and the efficiency may be compromised. 
Apart from the curiosity and the possibility to investigate basic laws of early embryo development, during the past years chimaerae have become very important tools of transgenic embryo production. The key from this point of view is the embryonic stem cell, a special type of cells that can be isolated from very few mammalian species (including some strains of mouse and most probably primates including humans). These cells can be kept in undifferentiated stage infinitively under special circumstances, but can be differentiated into all initial layers (endoderm, ectoderm, mesoderm) and all cell types of an adult organism. Accordingly, directed genetic modification can be performed in these cells (including homologous recombination, knockout, knockin modification, etc.), then the cell with appropriate modification can be selected and proliferated. Colonies of these embryonic stem cells can be used for chimaera formation. In normal situation the more viable blastomeres would overgrow the embryonic stem cells. However, if the blastomeres are made developmentally handicapped (for example cells of tetraploid embryos, produced by fusion of the two blastomeres after the first cleavage), the embryonic stem cells will form the fetus, and the blastomeres of the original embryo will only contribute in the formation of extraembryonic tissues. Accordingly, the whole embryo will be genetically modified, and in contrast to the microinjection, this modification can be delicately designed.

The unfortunate disadvantage of this approach is that it can only be applied to mice (and probably in some primates). So far, in spite of repeated extensive attempts, no embryonic stem cells were isolated from domestic animals. Accordingly, in these species another method was required to perform directed genetic modification.

\subsubsection{Somatic cell nuclear transfer}

This method is both the ultimate application of oocyte and embryo manipulation and (so far) the most radical intervention in mammalian embryology. Although the method has been established to create directed genetic modification in domestic animals, its success has basically changed the (preexisting) laws of developmental biology, and created a lot of moral controversies and legal problems.

The idea is simple. The chromatin (the nuclear DNA) is removed from a metaphase II, fully matured oocyte, and replaced with the nucleus of a partially or fully differenciated somatic cell of an adult individual, newborn, or fetus. Subsequently the reconstructed embryo is subjected to a procedure called activation to start cleavage and subsequent embryo development.

As referred earlier, a similar technique has (almost entirely) successfully used in frogs (but the full competence of adult cells was never entirely proved). In mammals, experiments of Willadsen have proved that early embryo cells (up till the 32, in some instances 64 cell stage) preserve the ability to govern full term development from the very beginning. However, till the middle of the 90's, the 
overwhelming majority of developmental scientist was entirely convinced that such develoment is impossible to achieve by using a terminally differentiated mammalian cell as donor.

Retrospectively, it is quite strange that for ten years after Willadsen's work nobody tried to use somatic cells for nuclear transfer. Probably some research group did, but abandoned experiments after the first frustrating results. However, based on their excellent embryonic cell cloning and embryo culture system, the Scottish group lead by Ian Wilmut and Keith Campbell eventually succeeded. The first somatic cell cloned animal, a sheep called Dolly was cloned from a mammary gland cell, and shortly after her birth she forced scientist to change their principles regarding the possibility to induce totipotence in a seemingly irreversibly differentiated cell, including blood cells, muscle cells, granulosa cells and cumulus cells.

Technically, the process is demanding and included the following steps:

1. Enucleation of the oocyte. Although the term is misleading (no definite nucleus is present in the MII phase oocyte) it is commonly used for the procedure. The technique is similar to the biopsy of a cleavage stage embryo and it is performed by using analogue approaches. The demanding part is to find the appropriate segment of the oocyte that contains the chromatin. Different strategies may be applied to achieve this goal, including staining of the chromatin with fluorescent dyes and exposition to UV light; unfortunately both the stain and the UV exposition may damage the oocytes. Another approach is to aspirate the area of the ooplasm that is adjacent to the first polar body. However, the polar body may migrate from the chromatin, so using it as an orientation point may be misleading. A third probability is to use some cytoskeleton relaxants (for example demecolcine) that may induce an extrusion cone on the surface of the oocyte, and this extrusion cone or the adjacent cytoplasm will contain the chromatin. Although this is quite an accurate approach, it works properly in some species where the extrusion cone is large enough to differentiate it from small blebs or fragments. If the extrusion is partial, it can be used as orientation point for enucleation performed with micromanipulators (chemically oriented enucleation). However, in the lucky - and so far exceptional - situation the separation may be complete and the enucleation does not require micromanipulation (chemically induced enucleation). Although the latter approach is very attractive, its efficiency is rather low, and only a few offspring were reported as the result of this procedure. Finally, in transparent oocytes including those of mice and primates, a special microscope optical system (Oosight) may help the identification of chromatin in the cytoplasm.

Compared to the different enucleation techniques, transfer of the new nucleus to the enucleated oocyte (also called cytoplast) has only a few standardized techniques. 1. direct injection of isolated nuclei (or even the whole somatic cells) into the cytoplasm (as the analogue of ICSI), or 2. subzonal injection (SUZI) of the whole somatic cell followed by electrofusion to unify the two cells. While the 
first procedure seems to be really successful in mouse, the efficiency is rather compromised in other species including domestic mammals. Moreover, it requires the use of the expensive Piezomicromanipulators. Subzonal injection and electrofusion is the preferred method that works with a relatively high consistency and efficiency in domestic and experimental mammals.

Electrofusion requires an impulse generator that produces both alternate (sinus) waves, or a single (but repeatable) direct current pulses. The first one is used to align the cytoplast-somatic cell pairs between the electrodes in the appropriate position ( $90^{\circ}$ to the direction of the current), while the DC current creates small holes of cell membranes. If some of these holes on the concomittant surfaces repair inappropriately, unifying the two cytoplasm through a narrov bridge, in several minutes teh birdge may widen and the two cells merge completely.

The same impulse may trigger activation, as well, however the more common way is to induce activation 1 to 4 hours afterwards, to ensure time for cytoplasmic factors to reprogram the nucleus of the somatic cell for the great task, to restart and govern development from the very beginning. Although the mechanism of this reprogramming is rather mysterious and researchers disagree if this transitional period is really needed, in most cases it seems to result in higher developmental rates both in vitro and in vivo.

Activation is not the subject of this chapter, accordingly only the basic mechanism is summarized here: an induced increase of $\mathrm{Ca}++$ level in the cytoplasm decreases the level of cytoplasmic factors (for example Maturation Promoting Factor, MPF) that arrest the cleavage of matured oocytes, then an additional treatment prevents extrusion of the second polar body and also synthesis of MPF and other proteins with similar effect. Accordingly, a 3 to $6 \mathrm{~h}$ window is opened for the oocyte to escape from the developmental block. The initial effect inducing calcium increase may be various (mechanical, chemical, electric, etc.), while the second factor (s) are usual chemical ones. In domestic species, the artificial activation may be very successful, leading eventually to as high as $50 \%$ blastocyst rates in cattle and 70 to $80 \%$ blastocyst rates in pigs.

Cloned embryos can be transferred into recipient animals exactly the same way as those produced by in vitro fertilization or derived after in vivo fertilization by flushing the embryos from the uterus.

Two major technical concerns are often emphasized regarding somatic cell nuclear transfer. The first is the low overall efficiency, the other the high number of developmental abnormalities. However, during the past 10 , especially 5 years, the efficiency has increased radically in domestic species including cattle and pigs, as the result of small adjustments of the technology. Although the efficiency is still very low in other species (dogs, cats, etc.), it is predominantly the consequence of the insufficient knowledge regarding the requirements of the in vitro culture of oocytes and embryos in these species. Regarding developmental abnormalities, the same is applicable: while 10 years ago 
the "large offspring" or "cloned offspring" problem was a serious one that occurred in 5 to $10 \%$ of the cloned animals with various abnormalities, early deaths, stillbirths or abortions, lately these problems seem to be mostly eliminated in the commercially most important two species, cattle and pigs. According to the opinion based on the results of the best cloning laboratories, it is quite likely that the overall efficiency of somatic cell nuclear transfer will soon approach, maybe even surpass of that of in vitro fertilization in the given species.

On the other hand, there are some very promising facts related to somatic cell nuclear transfer. Firstly, the healthy clones seem to be really healthy, and their products (meat, milk) may not mean any risk for human consumption. Secondly, developmental abnormalities may still occur in the first generation, but their frequency is equal with the controls in the second generation, even if two cloned animals are mated. That proves the temporary, most probably epigenetic nature of the cloning-related problems, an means a good possibility to overcome these problems. Finally, even with the present efficiency, cloning may be used for many purposes, as a few animals may be enough to achieve the goals.

These goals have been summarized recently in many reviews, accordingly we only mention the most important ones in headlines:

1. Animal breeding purposes: cloning of animals with the best genetic traits (for example for sperm production in cattle)

2. Preservation of endangered species or breed, where only a few individuals are available

3. Production of transgenic domestic animals with directed genetic modification in the somatic cells - that will eventually occur in all cells of the embryo and offspring, and will be inherited to the next generation. The goals may include

a. To produce animals with improved genetic traits, better quality milk, etc.

b. To produce animals resistant for certain diseases, for example BSE.

c. To produce genetically modified domestic animals for biomedical purposes including i. Production of certain bioactive proteins (coagulation factors, etc.) in the milk, ii. Production of animals with low or no immunogenecity for organ transplantation to humans

iii. Production of animals containing genes of human diseases for models to study and to treat these diseases.

The frames of this chapter do not allow to discuss potential human applications including therapeutic or reproductive cloning. Arguments regarding these controversial issues change every day, depending on the actual discoveries and the political atmosphere in the given country. We suppose that during the next decade emotions regarding somatic cell nuclear transfer will calm down, 
and a rational approach will help to find its proper application (and also prevention of misuse) in the future science and industry.

\subsection{Are micromanipulators really needed?}

Earlier we have described the handicapped situation of technical support of embryology compared to other branches of science. Micromanipulators and related tools (sophisticated microscopes, lasers, piezo manipulators etc.) can be regarded exceptions: in this field ultimate solutions of technology are applied. Quite controversiall, the recent development in some techniques questioned the need of these sophisticated tools, and predict a restricted use to very special purposes.

For example embryo bisection as well as blastocyst biopsy can be easily performed by hand, with a piece of a sharp razor blade glued to a glass capillary. Embryos can be fixed to the plastic with scratching the smooth surface and with a light pressure of the blade. Then the embryos may be turned with the blade to the right position for bisection (halving for twin production, or cutting off a small piece of trophectoderm for biopsy), and with careful lowering the blade, the two parts can be easily separated. The efficiency of these procedures is not lower than those performed with micromanipulators, and the speed and costs of work are incomparably lower.

ICSI is a field where micromanipulators will be needed during the next decades. However, for assisted hatching, both chemical and mechanical (hand-hold) approaches may become more efficient than the present methods, by exposing shortly embryos to the appropriate solution that removes partially the zona, or by cutting a small window on the zona without touching the blastomeres.

Injection of the inner cell mass or embryonic stem cells into the blastocoel for chimaera production is now replaced with a simple procedure, where the zonae of precompaction stage embryos are completely removed and two embryos (or one embryo and a clump of embryonic stem cells) are placed in small holes made with special needles on the bottom of the dish. Aggregation of the two cell masses may occur in hours, and a single blastocyst is formed from the two groups of cells with different origin. These zona-free embryos may be handled exactly the same way as zona included ones, can be cryopreserved and transferred to recipient animals with high survival and developmental rates.

Finally, during the past 6 years an alternative technique has also been described for somatic cell nuclear transfer. Using the same principles as described in the previous paragraphs for chimaerae and biopsy, the zona of MII phase oocytes is removed by digestion, then an oriented bisection is performed for enucleation. To make pairing of cytoplasts and somatic cells in the absence of the zona pellucida, cytoplasts are shortly immersed into a biological glue (phytohaemagglutinin), then rolled over a single somatic cell. The cells attach to each other and can be subjected to electrofusion. 
Activation steps are identical to those used for zona-incuded reconstructed embryos, and further culture is performed in a modified (larger) version of the aggregation holes, in the well of the well (WOW) system. The efficiency of this cloning procedure is much higher than that of traditional somatic cell nuclear transfer; the costs are negligible; and it does not require a long learning period, can be acquired in a week by an average embryologist. This handmade cloning (HMC) procedure has been successfully used in cattle and pigs with high number of healthy offspring even after cryopreservation of the reconstructed embryos, and with some modification, offspring were also achieved in mouse, horse and sheep. Quite paradoxically, all instruments needed for handmade cloning were available before the 2nd World War, when Spemann outlined the "fantastic experiment" in his book, accordingly, with a little more knowledge about the need of in vitro mammalian embryology and a little more courage Dolly could be born 60 years earlier.

On the other hand, the handmade approach may replace traditional nuclear transfer, accordingly the need of micromanipulators for future cloning work (as well as in most areas in embryology where they are actually used) is questionable. One realistic possibility is that these expensive tools will only be used for purposes where they are indispensable (for example for ICSI), and other purposes will be achieved with simpler approaches, or by automation, that may be unavoidable for the 21 st century embryology.
Abbreviations
HMC - Handmade cloning
ICSI - Intracytoplasmic sperm injection
MIMIC - Micro-Injection into cytoplasm
PVP - Polyvinylpyrrolidone
SUZI - Subzonal injection 


\section{Transgenesis}

\subsection{Introduction}

Over the last three decades biotechnology has advanced to a level where it is generally feasible to make particular changes to the genome, and therefore to the expressed characteristics, of living organisms. The product of such a change is called a transgenic or a genetically modified organism (GMO). GMOs are created and/or modified in the laboratory to amplify desired characteristics which are beneficial to mankind. However, improvement of desired characteristics has been traditionally undertaken through conventional breeding methods. In traditional breeding, scientists often introduce unknown genes into a species en masse by hybridizing them with a related species with a desirable trait. Genetic engineering, by contrast, involves splicing no more than a few well characterized genes into the organism and hence seems less drastic. Gene transfer is of particular value in those species, where long life cycles reduce the value of classical breeding practices for rapid genetic modification. Transgenic (genetically modified) animals are just one in a series of developments in the area of biotechnology. These terms now include the use of living organisms or their parts to make or modify products, to change the characteristics of plants or animals, or to develop micro-organisms for specific uses. The novel uses of biological techniques such as recombinant DNA techniques, cell fusion techniques, mono and polyclonal antibody technology and biological processes for commercial production have altered traditional distinctions and methods. Genetic manipulations at the level of DNA have also changed long held views as to what is considered to be animal, plant and human. In turn, these changes have made it more difficult to evaluate the ways in which animals are used and have obscured distinctions between pure and applied research. Consideration of the acceptability of creating specific transgenic animal strains or genetic manipulation involving interchanging DNA between species and kingdoms could be a simple animal care issue or a societal decision. The following is an attempt to show what the ability to create transgenic animals or engage in other forms of DNA manipulation means in terms of traditional ACC functions, not forgetting that this impacts on wider considerations of human responsibility for the welfare of other life forms. The creation of transgenic animals is resulting in a shift from the use of higher order species to lower order species, and is also affecting the numbers of animals used. This shift in the patterns of animal use is being monitored by the CCAC through the use of the Animal Use Data Form. An example of the replacement of higher species by lower species is the possibility to develop disease models in mice rather than using dogs or non-human primates. In the long term, a reduction in the number of animals used, for example to study human diseases, is possible due to a greater specificity of the transgenic models developed. On the other hand, the success of the method has led to using its potential for 
investigating a wider range of diseases and conditions. The actual use of some species may be increased, in addition to the numbers of animals which are sacrificed as donors during the creation process. The potential of the technology has also made it possible to consider employing cattle, swine, sheep and goats as processing units to manufacture proteins or as organ donors. The complex interactive processes of living mammals are not reproducible in vitro. However, transgenic animals provide a means of evaluating genetic modifications in terms of anatomical and physiological changes in a complex system. Transgenic models are more precise in comparison to traditional animal models, for example the oncomouse with its increased susceptibility to tumor development enables results for carcinogenicity studies to be obtained within a shorter time-frame, thus reducing the course of tumor development in experimentally affected animals. However, models are not strict equivalents, so as with any other system care must be taken in drawing conclusions from the data.

The term transgenic animal refers to an animal in which there has been a deliberate modification of the genome - the material responsible for inherited characteristics - in contrast to spontaneous mutation. Foreign DNA (gene construct) is introduced into the animal, using recombinant DNA technology, and then must be transmitted through the germ line so that every cell, including germ cells, of the animal contain the same modified genetic material.

Other important area of transgenesis is production of transgenic cell cultures. These genetically modified mammalian, bacterial etc. cells represent powerful tool for basic and applied studies, manufacturing biological active substances (medicals, hormones, antisera etc.) and substitutes of damaged cells in science, medicine and pharma industry.

\subsection{Transgenic animals - history and necessity}

In the mid-1970s, several investigators infected mouse embryos with retroviruses and demonstrated that proviral DNA integrated into the genome and was passed to subsequent generations (germ-line transmission of foreign DNA). By the early-1980s the technology gained momentum with various laboratories producing transgenic mice by microinjecting genes into the pronucleus of a fertilized egg, which has been hailed as a seminal event in the development of animal biotechnology. The term "transgenic" describes animals carrying exogenous genes (foreign DNA segments - gene construct) integrated into their genome. Since that time this definition has been extended to include animals that result from the molecular manipulation of endogenous genomic DNA, including all techniques from DNA microinjection to embryonic stem (ES) cell transfer and "knock-out" mouse production. In 1982, thanks to Palmiter and co-workers, a photography of a giant transgenic mouse appeared on the cover of Nature, showing first visible phenotype in mice overexpressing rat growth hormone. In fact, early work of Richard Palmiter and Ralph Brinster (1986) related to growth 
performance and the dramatic phenotype of the growth hormone in transgenic mice subsequently influenced the emerging field in a most compelling manner for both basic and applied sciences. First reports of a transgenic rabbit obtained by pronuclear microinjection, which resulted in random insertional transgenesis, date back to 1985. And, by the 1985's sheep and pigs, goat (1991), cattle (1992) had joined the transgenic barn. Since then, the technology available has developed considerably with major turning points continuing to accelerate at an incredible pace and more significantly the reach of the technology also extended to poultry and aquatic organisms such as Atlantic salmon and zebra fish.

Today, transgenic animals embody one of the most potent and exciting research tools in the biological sciences. Transgenic animals represent unique models that are custom tailored to address specific biological questions. Hence, the ability to introduce functional genes into animals provides a very powerful tool for dissecting complex biological processes and systems. This has made it possible to explore the regulation of gene expression as well as the regulation of cellular and physiological processes. Significant uses of live transgenic mammals are in the arenas of agricultural, biological, biotechnological and biomedical sciences including production of pharmaceuticals and in the field of organ transfer from transgenic animals to humans. These require the ability to target gene expression and to control the timing and level of expression of specific genes. Experimental designs have taken advantage of the ability to direct specific expression (including cell types, tissue, organ type, and a multiplicity of internal targets) and ubiquitous, whole-body expression in vivo. Transgenic farm animals are important in human medicine as sources of biologically active proteins, as donors in xenotransplantation, and for research in cell and gene therapy. Typical agricultural applications include improved carcass composition, lactation performance and wool production, as well as enhanced disease resistance and reduced environmental impact.

\subsection{Vectors for gene transfer}

In practical terms, it appears reasonable to consider that a gene and ideally a transgene are composed of three distinct domains. One corresponds to the transcribed region from the cap site to the poly(A) signal. Another contains the promoter region, including the proximal regulatory elements and the more distal enhancers. The third domain, which is not so precisely defined, harbors the gene boundaries also considered as gene insulator. These elements seem to play an essential role in the control of gene and transgene expression but have a more limited effect, if at all, in cultured cells. The transcribed region of a gene consists of several domains having quite different functions:
a) 5' untranslated region
b) coding region 

c) introns
d) 3 'untranslated region
e) transcription terminators
f) construction of polycistronic vectors

The promoter region contain regulatory elements controlling transcription of gene. They often also contain the major elements giving the cell specificity of expression. The enhancers located upstream are not thought to participate directly in the formation of the transcription complex but rather it increases the frequency of transcriptional events.

Boundary regions. Most of the transgenes are very sensitive to position effects for their expression which often is low, not strictly specific of the promoter used, and not copy number dependent. Admittedly, enhancer of the host genes at the site of integration can influence transgenes and reduce their specificity of expression. Locus-control regions (LCRs) have been identified in longgenomic DNA fragments flanking several genes used to generate transgenics. Two strategies to obtain reliable expression of transgenes are being studied. One relies on the use of vectors containig long genomic DNA fragments. The other is based on the addition of LCRs or insulators to conventional vectors.

\section{Types and Use of vectors}

\section{Cosmids}

Cosmids are hybrid vectors, 4-5kb in legth, that encompassed elements derived from bacteriophages and plasmids. These elements include:

a) an origin of replication which often derives from a phage to limit the overgrowth of recombinant vectors carrying small inserts

b) a selectable marker to identify transformed bacteria

c) one or two cos-sites that comprise a bacteriophage cos-end alongside the adjacent 200bp to allow the in vitro packaging of the recombinant DNAs during cloning process

d) unique cloning restriction site with rare restriction sites surronding it to allow the subsequent release of inserts from the vectors.

\section{YACs}

Transgenesis has proven useful in a variety of biological, medical and biotechnological applications and is considered a major breakthrough in the generation of transgenic animals. Yeast artificial chromosomes (YACs) are eukaryotic cloning vectors capable of the stable maintenance of 
genomic fragments of DNA larger than $1 \mathrm{Mb}$. YACs are unique as cloning vectors due to their huge cloning capacity and their unlimited potential for targeted modifications of incorporated genomic inserts. In several studies involving mice, YAC transgene expression was found comparable to that of endogenous levels and largely determined by transgene copy number. In addition, positionindependent expression has also been reported in YAC constructs stably transfected in cells. The generation of transgenic animals with YACs has been extended to other mammals with comparable success. To date, transgenic pigs (1995), rabbits (1996) and rats (1997) have been generated with YAC transgenes. However, the use of YACs is associated with several disadvantages, including insert chimaerism, insert instability, rearrangements and potential combination with endogenous yeast chromosomes that can make difficult their efficient purification for microinjection or transfection into ES cells.

\section{BACs and PACs}

To overcome these problems several other artificial chromosome-type vectors have been developed and have become popular. These include bacterial artificial chromosomes (BACs) and P1 bacteriophage-derived artificial chromosomes (PACs). PACs have been successfully used to generate transgenic mice and zebra fish in order to study long-range genomic interactions with the help of reporter genes. Similarly, BACs have also been applied in a wide variety of studies including molecular complementation of mutations, in vivo studies of gene function, analysis of gene dosage, and the identification and analysis of regulatory sequences found at long distances. Furthermore, BACs have been evaluated for their potential to improve mammary gland transgenesis and for the production of recombinant proteins in the milk of transgenic animals. Mammalian artificial chromosomes carrying reporter genes can be efficiently transferred into bovine embryos by microinjection and can sustain reporter gene (GFP) expression during early embryonic development and extended embryo outgrowth culture.

As a matter of fact, the use of YACs, BACs and PACs in transgenesis has allowed the discovery of new genes by complementation of mutations, the identification of key regulatory sequences within genomic loci that are crucial for the proper expression of genes and the design of improved animal models of human genetic diseases. Recently it has been noted that the generation and analysis of transgenic animals carrying artificial chromosome-type transgenes will be fundamental to functionally identify and understand the role of new genes, included within large pieces of genomes, by direct complementation of mutations or by observation of their phenotypic consequences. 


\subsection{Gene transfer}

Horizontal gene transfer (HGT), also called as Lateral gene transfer (LGT), is a process in which an organism transfers genetic material to another cell that is not its offspring. By contrast, vertical transfer (VGT) occurs when an organism receives genetic material from its ancestor, e.g. its parent or a species from which it evolved. Most thinking in genetics has focused on the more prevalent vertical transfer, but there is a recent awareness that horizontal gene transfer is a significant phenomenon. Artificial gene transfer is a form of genetic engineering. Genetic engineering, recombinant DNA technology, genetic modification/manipulation (GM) and gene splicing are terms that are applied to the manipulation of genes, generally implying that the process is outside the organism's natural reproductive process. It involves the isolation, manipulation and reintroduction of DNA into cells or model organisms, usually to express a protein. Gene transfer involves two distincly separate processes. The first step in the process must provide a mechanism by which genetic information can be transported from extracellular space, across biological membranes, and into the nucleus so that the incoming genetic information co-migles with the genome of the target organism. The second step in the process affords a means for the new genetic information to become part of the target genome.

The mutual contributions of developmental biology and genetic engineering permitted rapid development of the techniques for the creation of transgenic animals. DNA microinjection, the first technique to prove successful in mammals, was first applied to mice (Gordon and Ruddle, 1981) and then to various other species such as rats, rabbits, sheep, pigs, birds, and fish. Two other main techniques were then developed: those of retrovirus-mediated transgenesis (Jaenisch, 1976) and embryonic stem (ES) cell-mediated gene transfer (Gossler et al., 1986). Since 1981, when the term transgenic was first used by J. W. Gordon and F.H. Ruddle (1981), there has been rapid development in the use of genetically engineered animals as investigators have found an increasing number of applications for the technology.

Gene transfer can be realized through:

a) the gonads (ovary and testis)

b) the germ cells (sperm and oocyte)

c) the fetilized eggs and embryo

d) the somatic cells

\section{a) Methods of transgenic animals creation through the gonads}

The possibility of transfecting spermatogonia in situ via infusion of transgenes into semiferous tubules or transfection of germ cell precursors in vitro followed by transplantation into host testis was tested. It was demonstarated that testis-derived cells transplanted into the testis of infertile males 
could populate the host testis, generate sperm, and produce offspring. Presumably the next step would be to transfect the testis-derived cells before transplantation.

\section{b) Methods of transgenic animals creation through the germ cells}

In germline gene transfer, the parents' egg and sperm cells are changed with the goal of passing on the changes to their offspring. Germline gene transfer is not being actively investigated, at least in larger animals and humans, although a great deal of discussion is being conducted about its value and desirability. Since 1989, a new method for the production of transgenic animals has been available, namely sperm-mediated gene transfer (SMGT), based on the intrinsic ability of sperm cells to bind and internalise exogenous DNA molecules and to transfer them into the oocyte at fertilisation. The major benefits of the SMGT technique were found to be its high efficiency, low cost and ease of use compared with other methods. Furthermore, SMGT does not require embryo handling or expensive equipment. Sperm-mediated gene transfer could also be used to generate multigene transgenic pigs that would be of benefit as large animal models for medical research, for agricultural and pharmaceutical applications and, in particular, for xenotransplantation, which requires extensive genetic manipulation of donor pigs to make them suitable for grafting to humans. Three studies that combine sperm-mediated gene transfer with the other methodology are a case in point:

$>\mathrm{SMGT}+\mathrm{REMI}$ (restriction enzyme-mediated integration)

SMGT + electroporation (electroporation DNA into sperm before performing SMGT)

$>\mathrm{SMGT}+\mathrm{mAb}$ (antibody to associate transgene with sperm before surgical oviduct insemination)

\section{c) Methods of transgenic animals creation through fertilized eggs or embryos}

For practical reasons, i.e., their small size and low cost of housing in comparison to that for larger vertebrates, their short generation time, and their fairly well defined genetics, mice have become the main species used in the field of transgenics. There are several principal methods used for the creation of transgenic animals.

1) DNA single microinjection

This method involves the direct microinjection of a chosen gene construct (a single gene or a combination of genes) from another member of the same species or from a different species, into the pronucleus of a fertilized ovum. It is one of the first methods that proved to be effective in mammals. Microinjection in mice and rabbit eggs is relatively simple, thanks to better visualization of pronucleus (fig. 8.1). However, this does not hold good for embryos of rats and other higher species of animals, where it is much more complex. Total amount of microinjected DNA is about 1-2 pl, 
which at a concentration of 1-2 ng/ $\mu 1$ represents around 200-400 copies of DNA, depending on the type of gene construct though. Eggs are fixed on one side with the help of holder pipette and are microinjected from the other end with the help of microinjection syringe of several micrometers in size automatically or using manual microinjector. Microinjection process causes a visible increase in the volume of the injected pronucleus. After microinjection followed by short time or long time embryo culture under in vitro conditions, embryos are transferred to oviduct or uterus of the hormonally treated female or using vasectomized males. Most of the gene constructs were prepared using cDNA for the studied gene. Under ideal circumstances and thanks to promoter, targeted expression of the gene in the desired tissue at sufficient level in proper time was achieved and thereby the ability of transgenic individual to carry the integrated gene to offspring confirmed. Animal accounts for a model organism for the purpose of transgenesis into which it is possible to introduce and integrate gene constructs of different sizes ranging from 10-20 kb using plasmids to about 250$300 \mathrm{~kb}$ using YAC, BAC and MAC vectors. The insertion of DNA is, however, a random process, and there is a high probability that the introduced gene will not insert itself into a site on the host DNA that will permit its expression. A major advantage of this method is its applicability to a wide variety of species.

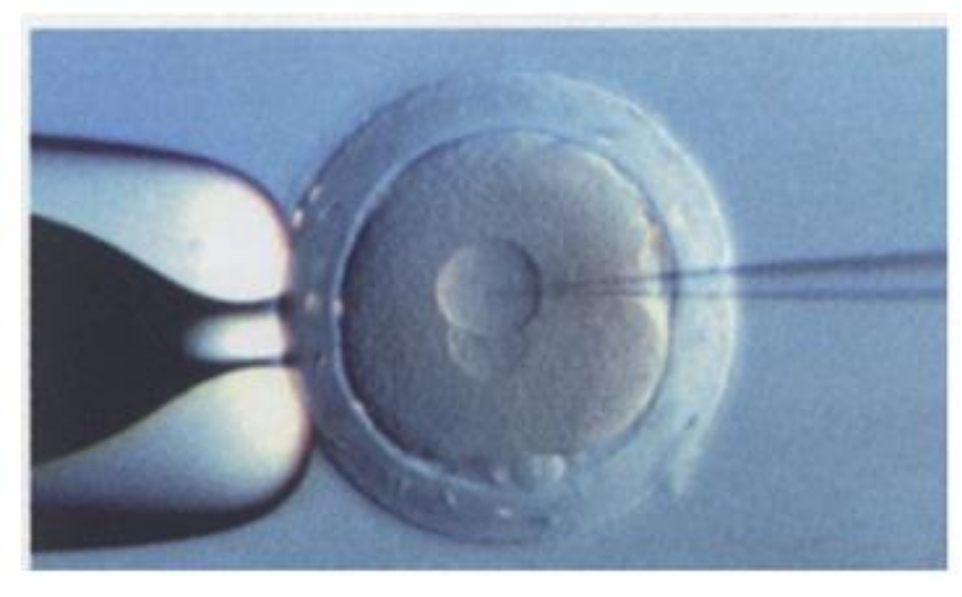

Figure 8.1: Gene transfer by microinjection of DNA into pronucleus

\section{2) DNA double microinjection}

On the same principle as microinjection of foreign DNA into pronucleus lies the method which facilitates the transfer of foreign gene into both the pronuclei of fertilized egg, which was successfully used for production of transgenic mice (Kupriyanov et al., 1998). This approach was extrapolated by Chrenek and his co-workers $(2003,2005 b)$ in a larger species thereby increasing the transgene integration efficiency upon microinjection of DNA into both pronuclei of rabbit embryos. In the case of double microinjection, degradation of embryos (lysis, fragmentation, irregular division) takes place 
based on the information as above. Obtained results also showed that double microinjection doesn't cause significantly higher percentage of embryo degeneration. Success of integration of foreign gene through this method was at least $30 \%$ more than that obtained by the previous method.

\section{3) Cytoplasmic injection}

However, using the established method of DNA-ligand-polylysine conjugate to transfect mammalian cells/DNA-transferrin-polylysine transfecting avain cells it was suggested that this might provide a means for cytoplasmic injection to be successful. The reported success of cytoplasmic injection of DNA/polylysine mixtures in producing transgenic animals also examined the effects of a number of DNA concentrations and lysine to phosphate ratios on transgenic efficiency.

Thus, cytoplasmic injection of DNA is possible by this method, although there is a loss of efficiency over the standard pronuclear injection procedure. This method allows the injection of gene into zygote even if the pronuclei are not visible (sheep, pigs, cattle). There is also the unexplored avenue that injection of DNA/polylysine mixtures anywhere in the zygote (including the pronucleus) may yield transgenic animals, thereby allowing trainee injectors a chance to be productive while acquiring the skills necessary to complete pronuclear injection proper.

4) Embryonic stem cell-mediated gene transfer

This method involves prior insertion of the desired DNA sequence by homologous recombination into an in vitro culture of embryonic stem (ES) cells using electroporation or liposomes. ES cells are isolated in culture from blastocyst-stage embryos, and embryonic germline (EG) cells are isolated from cultured primordial germ cell (PGC). Development of a stem cell line for a species that could be used as a recipient of the desired DNA and subsequent nuclear transfer of the stem cell nuclei or the incorporation of the stem cells into an embryo with the resulting birth of a chimeric animal are potential solutions to the inefficient incorporation of the injected DNA. ES cell-mediated gene transfer is the method of choice for gene inactivation, the so-called knock-out method. ES cells first were isolated from mouse embryos and since have been used as a model for mammalian embryogenesis and more recently for genetic manipulation (fig. 8.2). Due to their ability to integrate foreign DNA and thereafter differentiate into any and all tissues of a normal individual, ES cells are effective vehicles for genetic engineering and for creating laboratory animal models of human diseases. Despite considerable effort, progress towards isolation of ES cells from agricultural species has been slow. Some of the more promising results have been achieved with pigs. To date, production of embryonic stem cell lines has been much more difficult in livestock species than in mice. 


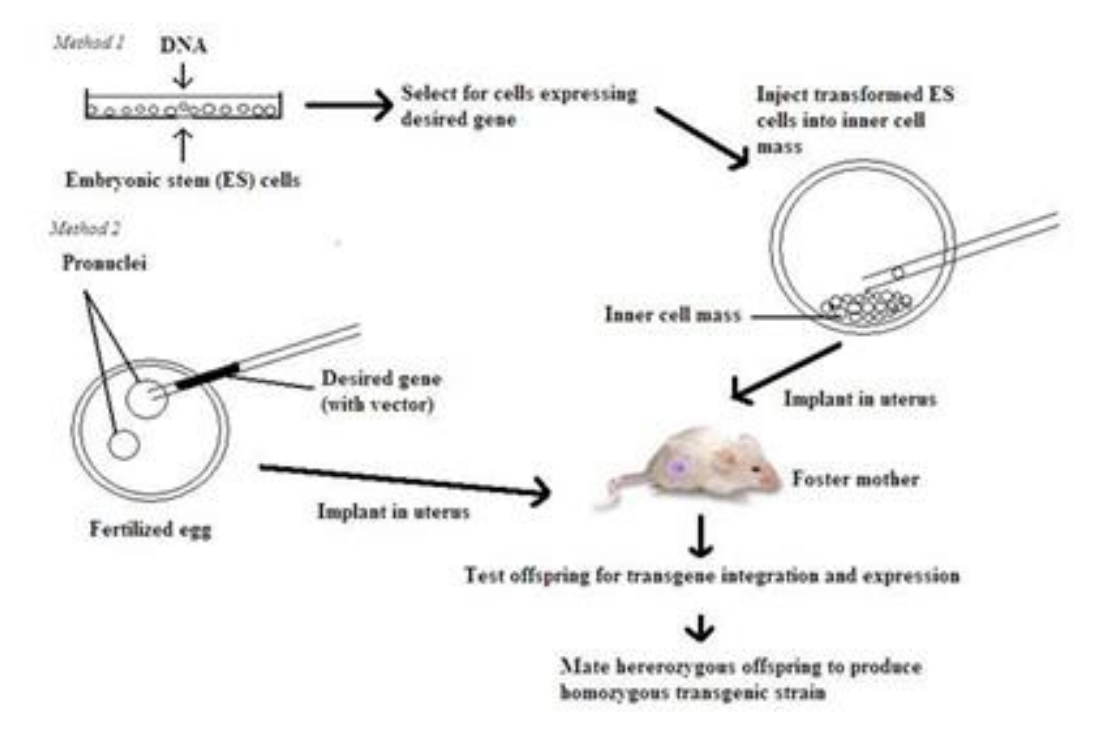

Figure 8.2: Embryonic stem cell-mediated gene transfer

5) Retrovirus (Adenovirus)-mediated gene transfer

To increase the probability of expression, gene transfer is mediated by means of a carrier or vector, generally a virus or a plasmid. Retroviruses are commonly used as vectors to transfer genetic material into the cell, taking advantage of their ability to infect host cells in this way. Offspring derived from this method are chimeric, i.e., not all cells carry the retrovirus. Transmission of the transgene is possible only if the retrovirus integrates into some of the germ cells. For any of these techniques the success rate in terms of live birth of animals containing the transgene is extremely low. Providing that the genetic manipulation does not lead to abortion, the result is a first generation (F1) of animals that need to be tested for the expression of the transgene. Depending on the technique used, the F1 generation may result in chimeras. When the transgene has integrated into the germ cells, the so-called germ line chimeras are then inbred for 10 to 20 generations until homozygous transgenic animals are obtained and the transgene is present in every cell. At this stage embryos carrying the transgene can be frozen and stored for subsequent implantation.

\section{6) Nuclear Transfer}

Nuclear transfer, which involves the transfer of each nucleus (genetically modified) from a multicellular embryo into an enucleated metaphase II oocyte (Fig. 8.3), has been developed in several species. Nuclear transfer has become an active field of research over the last decade, culminating in reports over the past two years of live offspring from differentiated cells of sheep, cattle, and mice. Dozens of laboratories are producing calves and lambs whose genetic parents are transgenic somatic cells, often derived from adults, transfected via electroporation. In spite of the fact that research 
orients towards modification of somatic cell genome through homologous recombination with the aim of producing so called "knock-out" (targeted gene is blocked) or "knock-in" (which refers to the integration of the transgene to specific site in the cell). After selecting the positive cells ("knock-out" or "knock-in"), these may serve as donor of nucleus mainly for the purpose of cloning. As the production of cloned (or transgenic) animals is demanding, combination of the techniques of transgenesis, cloning and in cases also chimerism allows achieving upto $30 \%$ better results. The principle depends on the utilization of transgenic somatic cells (or nucleus) for the purpose of cloning. Blastomeres isolated from cloned together with early transgenic embryos are subsequently used for the purpose for chimera. In this manner, chimeric cloned transgenic animals can be obtained.
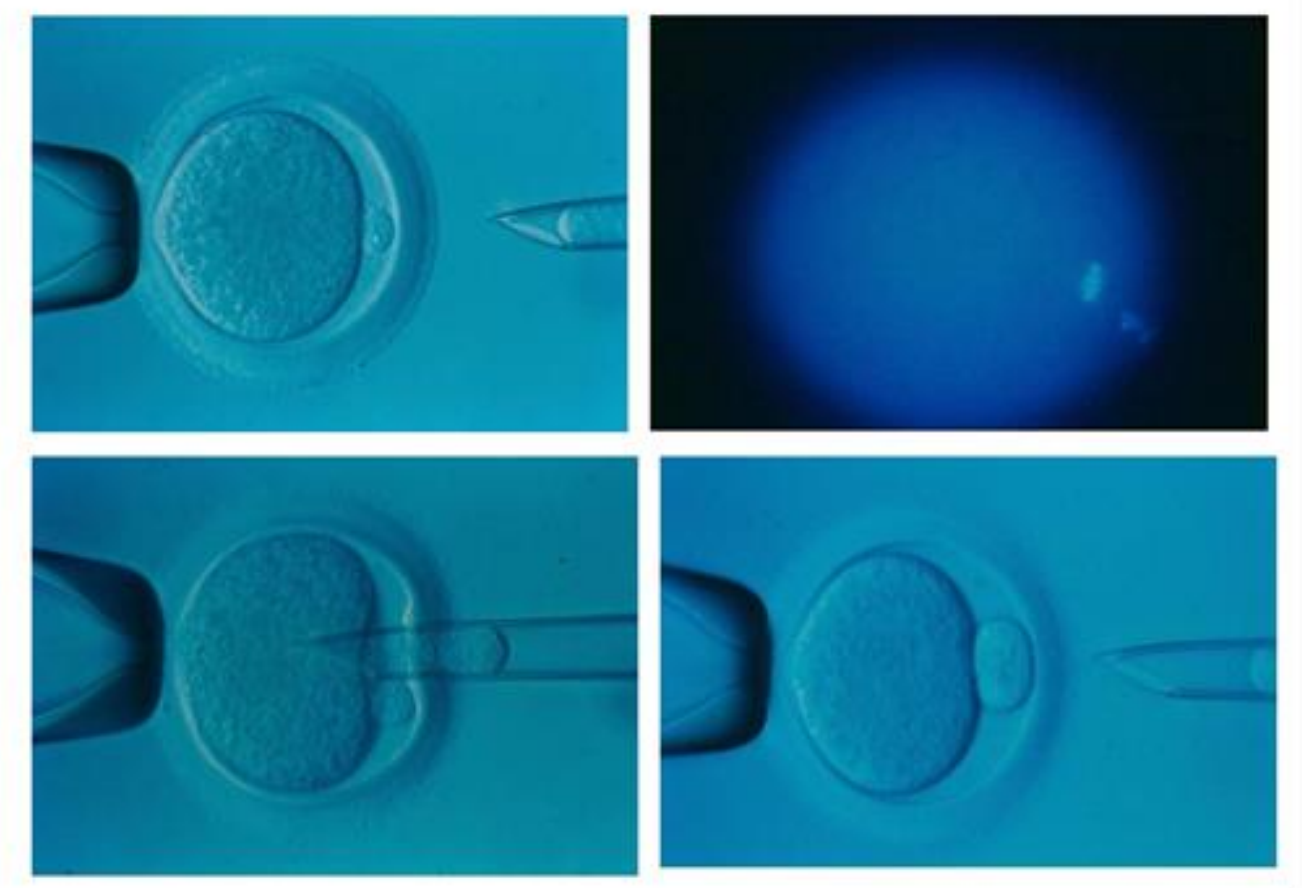

Figure 8.3: Princip of nuclear transfer

\section{d) Methods of transgenic animals creation through somatic cells}

In somatic gene transfer the recipient's genome is changed, but the change is not passed on to the next generation. Matsumoto and his colleagues (2001) developed a nonviral and plasmid-based method for arterial gene transfer by in vivo electronic pulse, using a newly designed T-shaped electrode. Using rabbit carotid arteries, they first optimized gene transfer efficiency, and firefly luciferase gene transfer via electronic pulse under 20 voltage (the pulse length: Pon time 20 mins, the pulse interval: Poff time 80 mins, number of pulse: 10 times) showed the highest gene expression. Electroporation-mediated gene transfer of E. coli lacZ with nuclear localizing signal revealed 
successful gene transfer to luminal endothelial cells and to medial cells. They concluded that in vivo electroporation-mediated arterial gene transfer is readily facilitated, safe and may prove to be an alternative form of gene transfer to the vasculature.

\subsection{Factors influencing efficient production of transgenic animals}

The influences of several factors on the efficiency of producing transgenic animals were statistically evaluated. One of these is the species the other is the age of recipients used (table 8.1 and 8.2). A higher survival rate of microinjected embryos was obtained after embryo transfer to younger recipients. The survival rate of microinjected embryos and the mean litter size of recipients increased as the number of embryos transferred per recipient. There exist a few factors, which may significantly influence the frequency of integration. Other factors are microinjection, followed by DNA concentration, lethality and insufficient cleanliness and purity of DNA. Improper microinjection, i.e., injection into cytoplasm instead of the pronucleus or damage of embryo during injection may decrease the efficiency of this method. As for DNA concentration, dilution of DNA may remain in the needle of injection pipette. It is necessary to prevent the infiltration of media from the slide alongside inside the injection pipette during the entire procedure, because this infiltration may result in dilution of DNA. Lethality is another important consideration because gene construct may have lethal effects on embryo. Lethal effect of gene construct is evident in desired or only in couple of newborn animals, in which the given gene construct was integrated. Insufficiently clean DNA may negatively influence the success of integration. During the separation of insert from the plasmid, use of agarose may drastically reduce the frequency of the integration. This is why it is preferable to carry out the separation of centrifugation with the help of sucrose gradient.

According to Wall and Siedel (1992), it is necessary to synchronize time of microinjection with cell cycle eggs (developmental stage of pronucleus), because integration process takes place depending on DNA replication. From the viewpoint of obtaining as high frequency of integration as possible, it is preferable to inject embryo before or in the beginning of DNA replication. Wall (1996) stated that success of transgene integration is low and ranges around 1\% in farm animals (cattle, sheep, pigs), which is verified experimentally with transgenic pigs, where the success of integration was $0.24 \%$. In case of laboratory animals, the success ranges between $1.5-20 \%$ depending on the species and type of gene construct. 
Table 8.1: Efficiency of transgenic animal production (Wall, 1996).

\begin{tabular}{|c|c|c|c|}
\hline Species & $\begin{array}{c}\text { Number of } \\
\text { embryos } \\
\text { used }\end{array}$ & $\begin{array}{c}\text { Number of } \\
\text { born animals }\end{array}$ & $\begin{array}{c}\text { Efficiecy of } \\
\text { transgenic } \\
\text { animal (\%) }\end{array}$ \\
\hline \hline mouse & 12314 & 1847 & 2.6 \\
\hline \hline rabbit & 1907 & 218 & 1.5 \\
\hline \hline rat & 1403 & 353 & 4.4 \\
\hline \hline cattle & 1018 & 193 & 0.7 \\
\hline \hline pig & 19397 & 1920 & 0.9 \\
\hline \hline sheep & 5424 & 556 & 0.9 \\
\hline \hline
\end{tabular}

Microinjected DNA usually integrates only at one site, or a very limited number of different sites, in individual embryos. The number of copies that integrate is highly variable, ranging from just one copy to as many as 200 . When multiple copies integrate, they are almost always found linked in a tandem head-to tail array at site of integration, which may influence efficiency of transgenic animal production.

Another important factor is the stability of transgene transmission to offspring. Voss et al. (1990) evaluated factors responsible for low efficiency - zygotes of two species, varying intensities of microscope light, different qualities of injection pipettes, and tested six different genes for their influence on the efficiency of pronuclear gene injection for the production of transgenic rabbits and mice. They found that rabbit zygotes were less sensitive to mechanical manipulation during injection than mouse zygotes and exposure of zygotes to a higher microscope light intensity significantly reduced their cleavage rate, while a lower intensity did not. Implantation rates also varied between $2.9 \%$ and $23.1 \%$ depending on the gene used. Popova et al. (2004) studied the factors affecting the efficiency of transgenic technology in rats and concluded that the main detrimental factor in the microinjection of rat zygotes is the introduction of solution in the pronucleus. They also stated that overnight culture of zygotes between microinjection and oviduct transfer does not increase the efficiency of transgenic rat generation. Further, the majority of transgenic animals generated by pronuclear microinjection appears to be mosaic in both somatic cells and germ cells for the pattern of DNA integration (Wall, 1996). This could be caused by late integration of transgenes during embryonic preimplantation development. Chrenek et al. (1998), while studying the factors 
influencing the developmental potential of cultured rabbit zygotes observed that the hormonal treatment of rabbit donors resulted in a doubling of the number of recovered ova per donor when compared with the non-treated group. However, the quality of recovered zygotes (presence of both pronuclei) was significantly better in the non-treated group. In other studies, Chrenek and co-workers $(1998,2005)$ successfully used superovulations to enhance the number of embryos and they also reported that double microinjection of DNA (into both pronuclei of rabbit embryos) is more efficient (in regards to transgene integration) to produce a large number of transgenic animals than single microinjection. Makarevich et al. (2005) suggested that apoptosis is one of the major causes of the low quality and viability of microinjected embryos as microinjection-derived cleavage-arrested embryos exhibited a poorly developed nucleolus, swollen mitochondria, several lipid vesicles, an extensive area with dispersed electron-dense material as well as nuclear membrane bleeding and numerous electron-dense bodies. Other general factors believed to influence the production of transgenic animals (Table 8.2) include:
a) breed
b) availability of fertilized eggs for microinjection
c) evaluating the obtained eggs
d) culture of embryos
e) microinjection of eggs
f) embryo transfer 
Table 8.2: Some factors influencing efficient production of transgenic animal (Wall, 1996). +++ excelent,++ good, + weak

\begin{tabular}{|c|c|c|c|c|c|c|}
\hline & mouse & rat & rabbit & pig & $\begin{array}{c}\text { sheep and } \\
\text { goat }\end{array}$ & cattle \\
\hline Litter size & $8-12$ & $8-10$ & $8-12$ & $6-10$ & $1-3$ & 1 \\
\hline Superovulation & ++ & ++ & ++ & + & + & + \\
\hline $\begin{array}{c}\text { Chirugical } \\
\text { embryotransfer }\end{array}$ & yes & yes & yes & yes & usually & no \\
\hline $\begin{array}{c}\text { Cutlture in in } \\
\text { vitro }\end{array}$ & +++ & + & +++ & ++ & ++ & + \\
\hline $\begin{array}{l}\text { Pronucleus } \\
\text { visualization }\end{array}$ & +++ & + & +++ & - & + & - \\
\hline $\begin{array}{l}\text { Integration } \\
\text { efficiency }\end{array}$ & +++ & ++ & ++ & + & + & + \\
\hline $\begin{array}{c}\text { Number embryos/ } \\
\text { superovulation }\end{array}$ & $15-40$ & $15-30$ & $20-35$ & 15 & $8-15$ & 5 \\
\hline $\begin{array}{l}\text { Embryotransfer } \\
\text { efficiency \%) }\end{array}$ & 50 & 40 & 65 & 40 & 40 & 20 \\
\hline $\begin{array}{l}\text { Number of } \\
\text { newborn \% }\end{array}$ & 15 & 10 & 10 & 6 & 15 & 10 \\
\hline $\begin{array}{l}\text { Number of } \\
\text { transgenic } \\
\text { animals }(\%)\end{array}$ & 20 & 10 & 10 & 5 & 5 & 5 \\
\hline Pregnancy (days) & 20 & 20 & 30 & 114 & 150 & 270 \\
\hline $\begin{array}{l}\text { Adult age } \\
\text { (month) }\end{array}$ & 1.5 & 2 & 4.5 & 6.5 & 6.5 & 13 \\
\hline
\end{tabular}

\subsection{The use of transgenic animals}

A representative, but non-inclusive, list of purposes for which transgenic animals have been used indicates the wide ranging application of this technology: 
a) in medical research, transgenic animals are used to identify the functions of specific factors in complex homeostatic systems through over- or under-expression of a modified gene (the inserted transgene);

b) in toxicology: as responsive test animals (detection of toxicants);

c) in mammalian developmental genetics;

d) in molecular biology, the analysis of the regulation of gene expression makes use of the evaluation of a specific genetic change at the level of the whole animal;

e) in the pharmaceutical industry, targeted production of pharmaceutical proteins, drug production and product efficacy testing;

f) in biotechnology: as producers of specific proteins;

g) genetically engineered hormones to increase milk yield, meat production; genetic engineering of livestock and in aquaculture affecting modification of animal physiology and/or anatomy; cloning procedures to reproduce specific blood lines; and

h) developing animals specially created for use in xenografting.

Many uses have been developed and many more are forecast, particularly in three areas:

Models of human disease processes: Hundreds of transgenic rodent lines have been produced by introducing into the genome genetic sequences such as viral transactivating genes and activated oncogenes implicated in specific pathologies. The phenotype and regulatory parameters of the gene may then be evaluated in an animal model with a relatively short generation time. Also, normal rodent genetics and physiology are highly characterized. The predictability of many transgenic phenotypes permits the innovative testing of diagnostic and therapeutic agents while using a reduced population of experimental animals. The generation of novel cell lines from transgenic organs also promises to reduce the number of research animals required to evaluate a therapeutic compound. In addition, transgenic genomes may be created in which more than one transgene may interact, or in which a transgene may interact with an endogenous normal or mutated gene. The use of transgenic disease models in biomedical research promises to accelerate dramatically the development of new human diagnostic and therapeutic treatments. Transgenic rodent models have been characterized for several human diseases including cardiovascular disease, cancer, autoimmune disease, AIDS, sickle cell anaemia and neurological disease.

Modification of animal anatomy and physiology: The most controversial aspect of transgenic animal usage involves the "selective improvement" of species by the modification of the genome. 
Most often, foreign genes are added to the host genome, although selective deletion of specific genes or regions has been attempted.

Targeted production of pharmaceutical proteins: Another use of transgenic animals involves the biological production of valuable human protein enzymes, hormones and growth factors (Fig.8.4). These products may be recombinant or mutated, and collection of the functional protein from the animal employs tissue-specific regulatory DNA sequences, as in the strategy described below. Current techniques in the biotechnology industry use large-scale cell cultures to generate products in biological systems. Eukaryotic cells or bacteria which have taken up genetic expression sequences (or constructs) are cultured in nutrient medium which is continually replaced and from which the bioengineered product is refined. This medium must be correctly buffered and must be temperatureregulated and pathogen-free. The use of transgenic animals, particularly larger mammals, as bioreactors ("pharmaceutical pharming") is a cost-effective alternative to cell culture methods. Animals automatically supplement their body fluids with fresh nutrients, remove waste products, reliably regulate their internal temperature and $\mathrm{pH}$ and resist pathogens. By directing (or targeting) the expression of the transgene product so that it is produced by the secretory cells of the liver, lactating mammary gland or kidney, "pharmers" may collect and process body fluids with minimal effort. The mammary gland is probably the most promising target tissue because it produces large amounts of protein in a temperature-regulated fluid that may be collected daily in a non-invasive fashion. Transgenic animals are not only cost-effective bioreactors but, with the complex secretory cell types and organs of the mammalian organism, can perform much more complicated protein modifications than simply cultured cells.

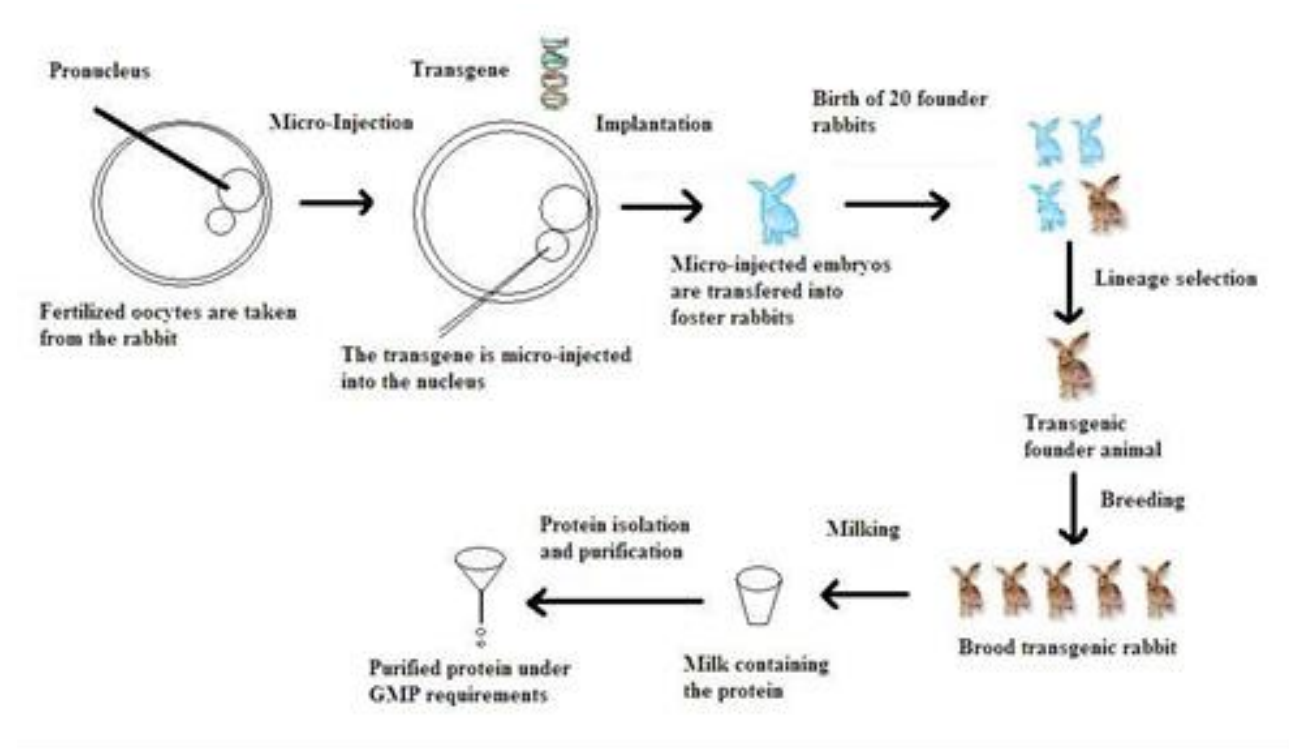

Figure 8.4: Production of recombinant proteins 
The different animal systems to produce recombinant proteins

1) Milk: Milk is currently the best available bioreactor. Extensive studies have shown that it can be the source of a variety of recombinant proteins, some of them being rather complex molecules. On the other hand, a certain number of failures occurred for various reasons. Some of them are purely technical such as the generation of transgenic animals. Other, such as protein maturation or secretion, is more fundamental.

2) Blood: Serum, which collects secretion from many tissues, may be the source of recombinant proteins. Although serum from transgenic animals is an abundant by-product of slaughterhouses it does not appear presently as a potential source for many recombinant proteins. Indeed, many proteins are poorly stable in serum and some of them may hamper the health of the animals.

3) Urine: Urine is an abundant biological fluid already used to prepare proteins such as gonadotropins for pharmaceutical use. The system allowed expression in urothelium may be useful if it happens that the foreign protein is maturated in a more appropriate manner in urothelium than in the mammary gland or if the side-effects of the protein are less deleterious for the animals.

4) Seminal plasma: Seminal plasma is relatively abundant biological fluid in some species and it can be easily collected. This system, as urine, may have specific advantages. In both cases, it is not known how complex proteins are matured and secreted.

5) Egg white: Egg white, as milk, is an abundant fluid containing huge amount of proteins and is being secreted out of the body. It is thus expected to be an excellent system to produce recombinant proteins. Yet, it has not been implemented so far, essentially for technical reasons.

6) Silk worm cocoon: The cocoon of insects contains large amount of a few proteins which form silk. From this point of view, this system shares a certain number of properties with milk and egg white. Silk worm can be easily reproduced into large number of individuals. It is therefore, a good candidate to be a living fermentor.

\subsection{Methods for detection of transgene integration and expression}

\section{Transgene integration}

The identification of a transgenic animal (transgene integration) is based on the detection of some aspect of the transgenne (Fig. 8.5). With the knowledge of the unique characteristics of the transgene, the investigator can utilize different methods for the identification and confirmation of transgene integration. The mechanism, based on which the injected DNA integrates to chromosomes, is unknown. Injected DNA often integrates at one site in chromosome, but a few integrations may also take place (Burdon and Wall, 1991). It is believed that the integration may take place due to 
chromosomal damage during injection and incorporation of tandem copies of gene mostly to one or more of such sites during repair. It may be expected that more the insertion sites, higher will be the effect on reproductive ability, which is caused by insertion mutagenesis, i.e. damaging the original genes in the site of incorporation.

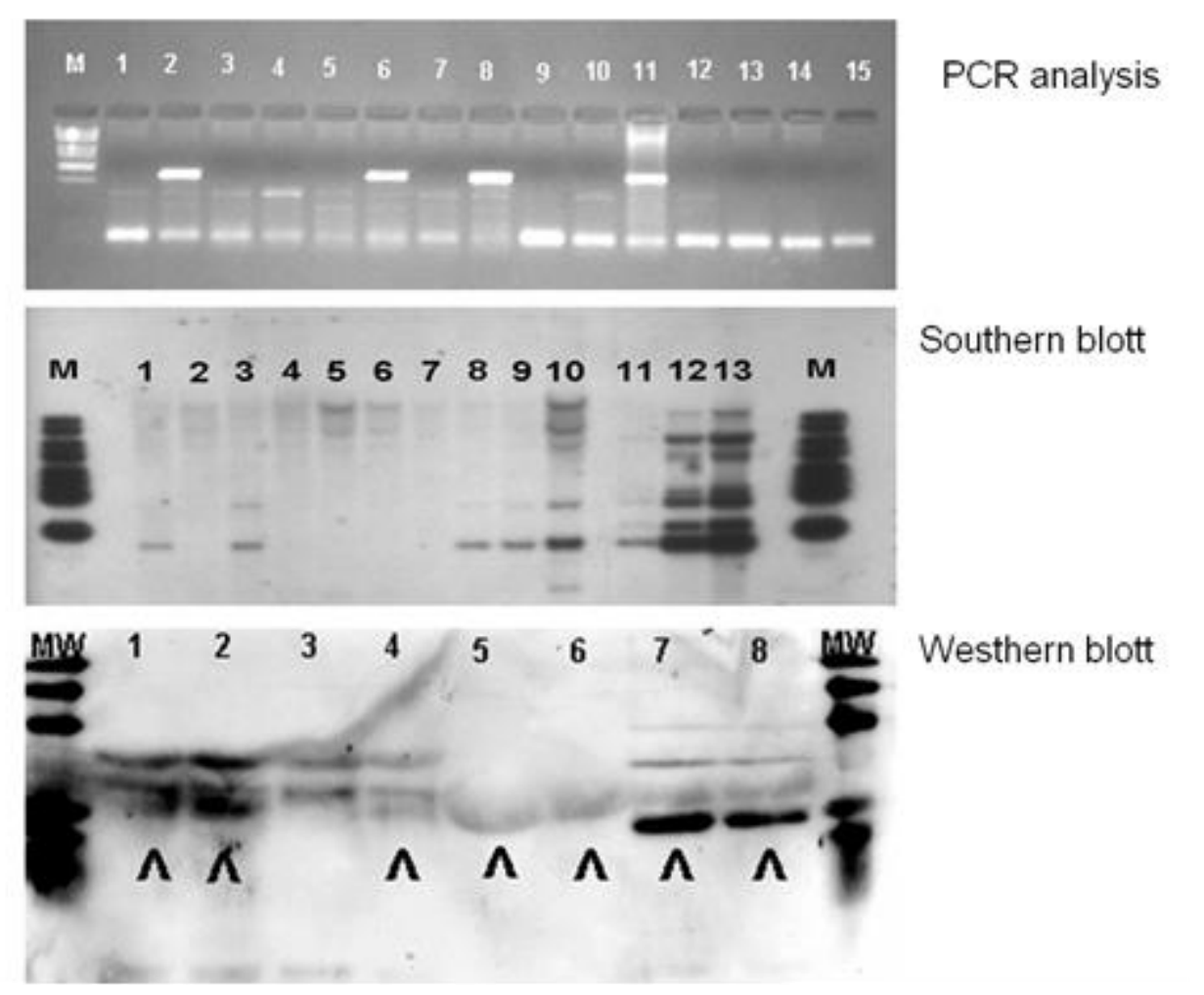

Figure 8.5: Detection of transgene integration and expression

1) Polymerase chain reaction (PCR). PCR is useful for detecting low-copy-number genetic target sequences by repeated rounds of amplification. Analysis by PCR has been applied to transgenic screening using DNA from variety of tissues including tail or ear biopsies, whole blood, and saliva. Briefly, the tissue is protease-digested, boiled, and used as a crude extract in the PCR reaction. The PCR reaction is in vitro amplification of specific DNA target sequences using specific primers during several cycles in a thermocycler machine. By this method we can detect only the presence of the integrated transgene in the genome of analysed animal.

2) Real-time PCR. Real-time PCR allows measure the quantity of amplified product.

3) Southern blot hybridization. DNA isolated from different tissues is digested with restriction enzyme, electrophoresed through an agarose gel, denatured, neutralized, blotted to a membrane such as nitrocellulose, and hybridized to a transgene-specific probe. By using known concentration of the injected DNA fragment (for example, equivalent to one, three, and nine copies per diploid genome), one should be able to estimate the copy number in each of the founder animals. 
4) Slot blot hybridization. The technique involves the direct application of the DNA sample to a solid support such as a nitrocellulose membrane and subsequent incubation of the membrane with a transgene-specific probe. Compared to Southern blot hybridization, slot-blot hybridization does not require cleavage of the input DNA with a restriction enzyme and, for that reason, does not require extensive purification of the DNA sample. As a result, the procedure will significantly reduce processing time and allow handling of many more samples.

5) Fluorescent in situ hybridization (FISH). The detection of nucleotidic sequences (specific for transgene) on a combed DNA molecule is peformed indirectly, by first hybridizing the seeked nucleotide sequences (the probes) with the combed DNA (also called the matrix DNA or target). If the probes are synthesized with incorporated fluorescent molecules or antigenic sites which can be recognized with fluorescent antibodies, the direct visualisation of the relative position of the probes is possible. Using this method it is possible to detect transgene intergation, copy of transgene and localisation of transgene on chromosome.

\section{Transgene expression}

Characterizing the expression of a transgene is a crucial step in all transgenic projects. In general, analysis of transgene expression is carried out on material derived from F1 generation offspring of founder animals. Frequently, the initial analysis to evaluate transgene expression involves a broad survey of a variety of tissues at the mRNA level, and next step at the protein level. Differential expression of foreign gene is given by:

a) regulatory sequences (promoter, enhancer)

b) site of integration

c) organization of solitary gene (presence or absence of introns)

d) environmental factors

Selection of regulatory sequences markedly influences the expression of genes. Regulatory sequences also define the tissue or organ, where the gene will express. The fact that presence of introns increases transcription efficiency of corresponding gene complicates its application because the segment of introduced DNA sequence of most genes is too long. Expression of foreign genes may also be effected by external factors, which is very important from the viewpoint of optimal utilization of transgenic animals. There are many methods to detect transgene expression in transgenic animals. 1) Reverse transcriptase-PCR (RT-PCR). RT-PCR can be very useful in detecting and quantifying gene expression. It is the most sensitive method. To carry out this assay, a specific "antisense" primer is allowed to anneal in solution to an RNA sample, and reverse transcriptase enzyme is used to convert 
a region of the mRNA of interest to cDNA. A second "sense" primer is then added to the sample along with a thermostable DNA polymerase.

2) Northern blot hybridization. Northern analysis shares with the slot-blot method the advantages of high sample throughoput and quantification. Another advantage of the northern technique is that it often allows one to measure the transgenic mRNA and its endogenous counterpart in the same sample, often in the same experiment.

3) In situ hybridization. In situ hybridization is an effective method for analyzing transgene expression at the cellular level. In this technique, microtome sections of either frozen or paraffin embedded tissues are fixed to a solid support, typically a glass microscopic slide. Following a series of treatment to fix, dehydrate, and permeabilize the tissue sections, a radiolabeled nucleic acid probe is allowed to hybridize to the mRNA in situ.

4) Western immunoblot analysis. This method involves separating total protein in different tissue extracts by sodium dodecyl sulfate - polyacrylamide gel electrophoretic (SDS-PAGE) analysis, transferring the protein from the polyacrylamide gel to a solid support (nitrocellulose membrane) with the aid of an electric field, and incubating the membrane with the appropriate antibody.

5) Radioimmunoprecipitation assay. PAGE analysis of immunoprecipitates obtained with the use of a specific antibody depends on the stability of the radiolabel of the antigene to be detected. In general, radioimmunoprecipitation has the advantage of providing size confirmation of the protein product that is specifically detected. It may also reveal whether the transgene product is properly modified, by using procedures such as two-dimensional gel electrophoresis or tryptic peptide analysis of the immunoprecipitate.

6) Reporter Gene. The inclusion of a reporter gene in the transgene construct is found to be helpful as it allows easy scoring of the integration and expression of the transgene. In recent years, reporter genes have been widely used in transgenesis, because they are easily detectable, with low background activities from endogenous gene product. Many reporter genes have so far been used. Of the various reporter genes, Escherichia coli $\beta$-galactosidase gene (lacZ) was commonly used. Some reporter genes entail the use of radioisotope like chloramphenicol acetyltransferase (cat). Firefly luciferase (luc) gene has been used as a reporter though its assay is very expensive. Horseredish peroxidase gene has also been used in transgenesis. There are several reports of use of green fluorescent protein (GFP) as a reporter gene in transgenesis. GFP absorbs blue light (492nm) and emits green fluorescence without any need for exogenous substrates or cofactors, and this characteristic is a great advantage of GFP as a marker (Fig. 8.6). Therefore, it is thought to be a useful marker gene for selecting transgenic embryos. GFP (including enhanced green fluorescent protein - EGFP) is a reporter useful as an alternative to tyrosinase minigene and other known reporter genes such as lacZ 
and luciferase gene, since expression of EGFP is noninvasive and does not require any cofactors, enabling observation without killing of samples.
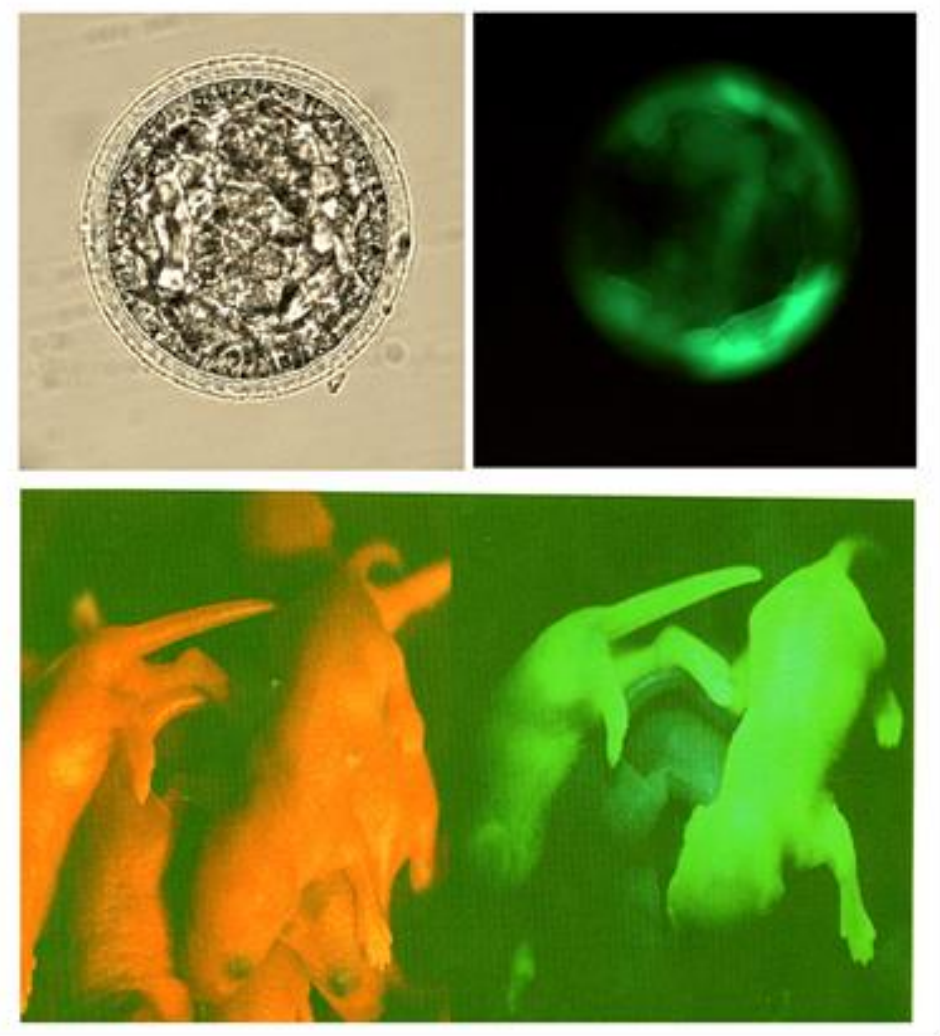

Figure 8.6: Detection of EGFP expression

Products of reporter genes can be used not only for identification, but also for selection and selective collection of transgenic cells (see below).

\subsection{Transgenesis in cell cultures}

\subsubsection{Purposes and areas of application}

Production and culture of transgenic animal, human, plant and bacterial cells have wide area of application within and without animal biotechnology (science, medicine, pharma industry etc.).

First, genetical modification of cultured cells was used for basic and applied studies of regulators and biological role of particular substances produced by cells. Such studies usually include (1) transfection of cells with gene constructs inducing changes in expression of particular substance (gene, group of genes, RNAs, peptides etc.) and (2) subsequent comparison of genetically modified 
and control cells to detect the consequence of changes in expression of studied substance and therefore to examine biological significance of this substance. Sometimes transgenesis is a sole useful way to change production of substances which could not be specifically influenced by other physiological and pharmacological methods (some enzymes, transcription factors etc.). In comparison with transgenic animals, studies of transgenic cells have some advantages:

> exact identification and manipulation with isolated target cells (in transgenic animals genetically modified cells can be distributed randomly in the organism, whilst expression of gene construct in particular cell requires use of cell-specific vector),

$>$ easy and efficient performance (simultaneous transfection of a large number cells in culture),

$>$ easy control of transfection and expression of studied substance (easy identification of target or marker substance in each cell by using microscopy, FAKS etc),

$>$ easier studies of cell characteristics in comparison with characteristics of whole animal,

$>$ easier manipulation with cells after transfection (use for transplantation, for study of effects of exogenous and endogenous factors, drugs etc.).

Second, genetically modified cells could be used for substitution of damaged cells and for improvement of physiological functions of organism. Genetical modification and subsequent transplantation of modified cells are successfully used for treatment of some genetical metabolic, immunological and reproductive disorders. Furthermore, genetical modification of immunocompetent cells with constructs, which improves their immunological and phagocytic properties, and introduction of such modified/activated cells into organism is an efficient tool for quick improvement of immunological nd inflammatory response of organism and its resistence to infections, injury and other damaged factors.

Finally, at present cultured genetical modified cells are the main source of manufacturing biological active substances (medicals, hormones, antisera etc.). During whole human history such biological active substances were collected and extracted from whole organisms (plants, animals) or their organs. Disadvantage of such approach was single use of source of desirable substance and low purity of obtained product. In 60-90's methods of culture of organs, tissues and cells, which allowed production of more pure substances in vitro and their collection from the culture medium were developed. The wide application of this approach was however limited by low viability of cells isolated from organisms. Threfore, at present for manufacturing biological active substances genetical modified lines of normal or malignant mammalian cells or procariotic ( bacteria, yeast) cells are used. Such cells are characterised by much better viability, reproduction and synthetic activity, than primary mammalian cells. 


\subsubsection{Manipulations with cell cultures}

Transfection of cells is usually performed by using electroporation, liposomes or retrovira (adenovira) as it was described above for production of transgenic animals. Due to large number (usually milliones) of cells in cultures used for transgenesis, less efficient microinjections and nuclear transfer in single cells are usually not used for genetical modification of large scale cell cultures. Genetical modification could be performed immediately after cell isolation before culture or alternatively after cell adaptation to culture conditions (for example, after formation cell monolayer). To assess success and efficiency of genetical modification of cell cultures, 6-48 hours after transfection, integration of gene constructs and their expression should be performed by methods described above. Markers of gene integration and expression should be used for subsequent selection of genetical modified cells for further use or storage.

Culture, selection and reproduction of small number of genetical modified cells (for research and medical purposes) can be performed in classical cell culture vessels (Petri dishes, culture plates, culture flasks). Large scale cultures (for industrial use) are usually performed by using specific facilities at microcarriers, in bioreactors etc.

Analysis of transgenic cells is necessary for validation expression of introduced gene, discrimination of transgenic and not transgenic cells, collection of transgenic cells and examination their functional properties. For identification and selection of small and medium amounts of genetical modified cells one use the same markers and methods as for transgenic animals. For discrimination of modified and not modified cells in large population one use mainly large-scale selection by using reporter genes-derived marker proteins sensitive to antibiotics, temperature and other selection factors, as well as automated immunological methods - fluorescence activated cell sorting (FACS) and magnetic cell separation (MACS).

By FAKS (other name - high throughout flow cytometry, HTFC) specific product is identified by antibodies against this product labeled with fluorescent marker. Thereafter cells are separated by using spefic equipment separation cells according to their fluorescence. By MACS antbodies are bind with magnetic microparticles, therefore cells, which bind these "magnetic" antibodies can be selectively collected by using magnetic beads, columns or separators. Functional properties of transgenic cells can be studied by standard cytological, biochemical and molecular biological methods. Presence of specific products in transgenic rabbit ovarian cells is demonstrated in Fig. 8.7. 

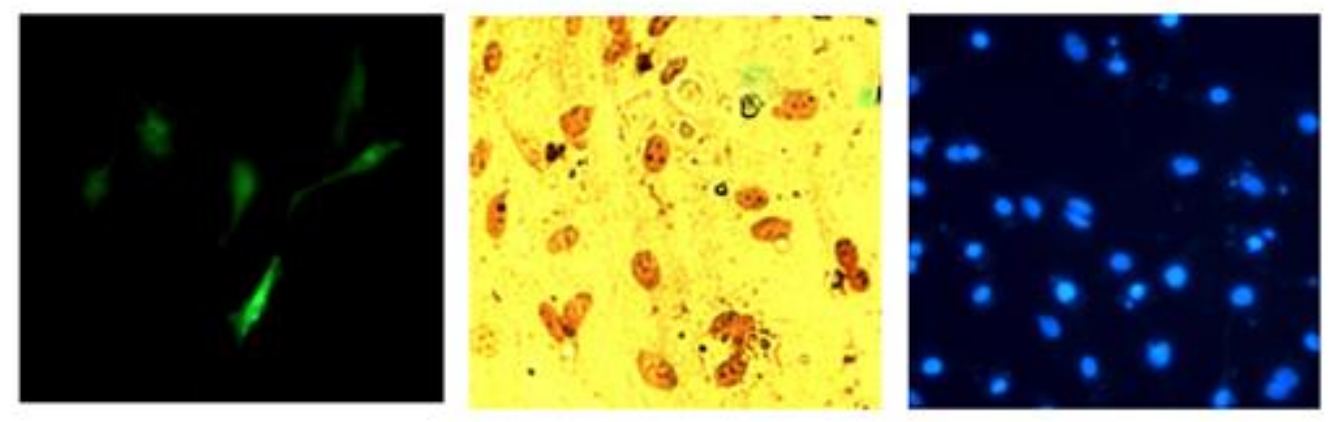

Figure 8.7: Cultured rabbit ovarian granulose cells transfected with cDNA constructs encoding enhanced green fluorescent protein (EGFP) and transcription factor p53 containing (a) EGFP in cytoplasm (fluorescent microscopy) (b) increased amount of transcription factor p53 in nuclei (immunocytochemistry by using antibody against $p 53$ labeled with horseradish peroxidase, staining with DAB, light microscopy) and (c) DAPI, marker for nuclear DNA (fluorescent microscopy) (A.V. Sirotkin, J. Petrak, A.V. Makarevich, unpublished data).

Abbreviations

BACs - bacterial artificial chromosomes

ES cell - embryonic stem cell

EG cell - embryonic germline cell

EGFP - enhanced fluorescent protein

FISH - fluorescent in situ hybridization

GFP - green fluorescent protein

GM - genetic modification/manipulation

GMO - genetically modified organism

HGT - horizontal gene transfer

LacZ - Escherichia coli $\beta$-galactosidase gene

LCRs - locus-control regions

LGT - lateral gene transfer

PACs - P1 bacteriophage-derived artificial chromosomes

PCR - polymerase chain reaction

PGC - primordial germ cell

RT - PCR - reverse transcriptase-PCR

YACs - yeast artificial chromosomes

SDS-PAGE - polyacrylamide gel electrophoretic

SMGT - sperm-mediated gene transfer

SMGT + REMI - restriction enzyme-mediated integration

VGT - vertical gene transfer 


\section{Nucleologenesis and nucleolotransfer in mammalian oocytes}

\subsection{Introduction}

The main goal of our chapter is to offer compact view on nucleolus and all its forms in consideration of current knowledge and to suggest the best way of its application in area of reproduction biology which can lead to an improvement development potential of early embryos used in veterinary or human medicine. The nucleolus is the intranuclear structure (organelle) in eukaryotic cells, usually located close to the nuclear membrane. It is a multifunctional organelle that is involved in rRNA synthesis and its posttranscriptional modifications, formation of ribosomes, cell cycle regulation, proliferation, chromosome segregation, reprogramming, common stress reaction, nuclear transport and many other functions. Interestingly, nucleoli in oocytes have several forms and functions, which vary during oocyte growth and early embryonic development. The typical differentiated nucleolus consists of fibrillar centers (FCs), dense fibrillar components (DFCs) and granular components (GCs) and can be found in somatic cells, stem cells, growing oocytes and in cells of advanced developing embryos (Fig. 9.1a).

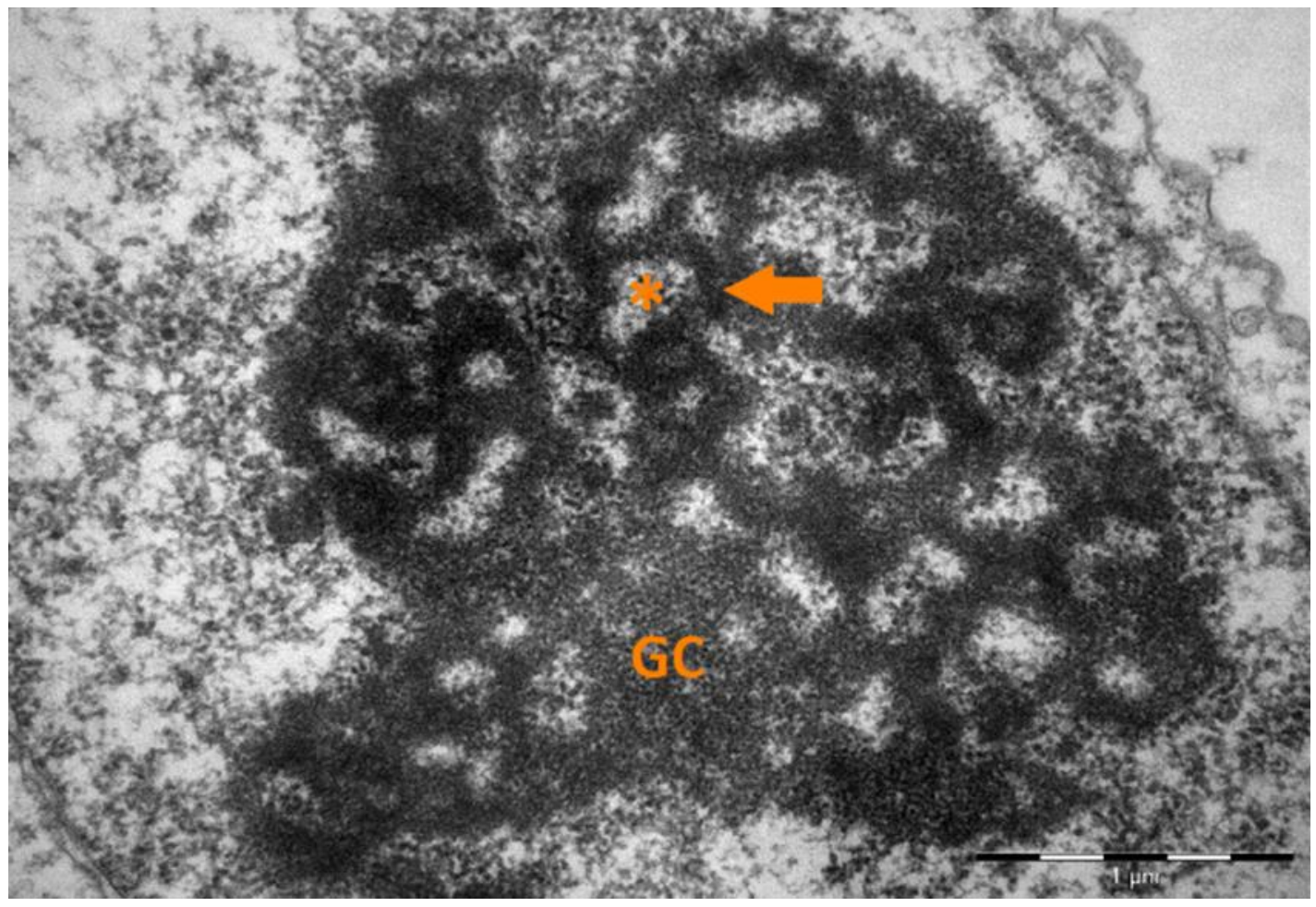

Figure 9.1a. An electronogram of nucleolar organization in somatic cell. The three nucleolar components are visible: the fibrillar centres (asterisks), the dense fibrillar component (arrow), and granular component (GC). Scale bar: $1.0 \mu \mathrm{m}$. 


\subsection{Nucleoli in oocytes}

Very profound morphological and functional changes can be seen in nucleoli during the oocyte growth. When the oocyte grows, the nucleolus has similar structure when compared to somatic cells, i.e. contains FC, DFC and GC with intensive RNA synthesis (Fulka, H. et al, 2015)). By contrast, in fully grown oocytes the nucleolar structure differs completely. The nucleoli of fully grown oocytes are visible as dense compact structures composed almost exclusively of fibrillar material and are called as nucleolus-like bodies (NLBs) (Fig 9.1b) (Lefèvre, B. ,2008).

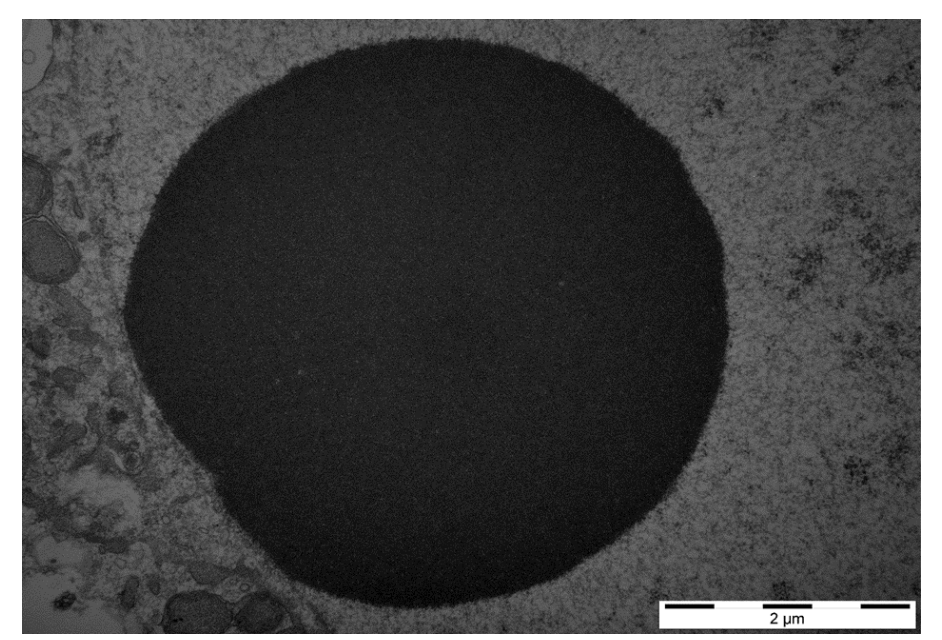

Figure 9.1b. An electronogram of NLB in fully grown porcine oocyte. Scale bar: $2.0 \mu \mathrm{m}$.

In mouse and human oocytes they are visible with conventional light microscopy (Fig. 9.2.). Accordingly, RNA synthesis decreases in nucleoli of this oocyte developmental stage (Fulka, J. Jr. et al., 2003). The fully grown oocytes do not synthesize ribosomal RNA, but synthesis of the heterogeneous nuclear RNA continues until the resumption of meiosis (Rodman, T. C., Bachvarova, R.,1976).

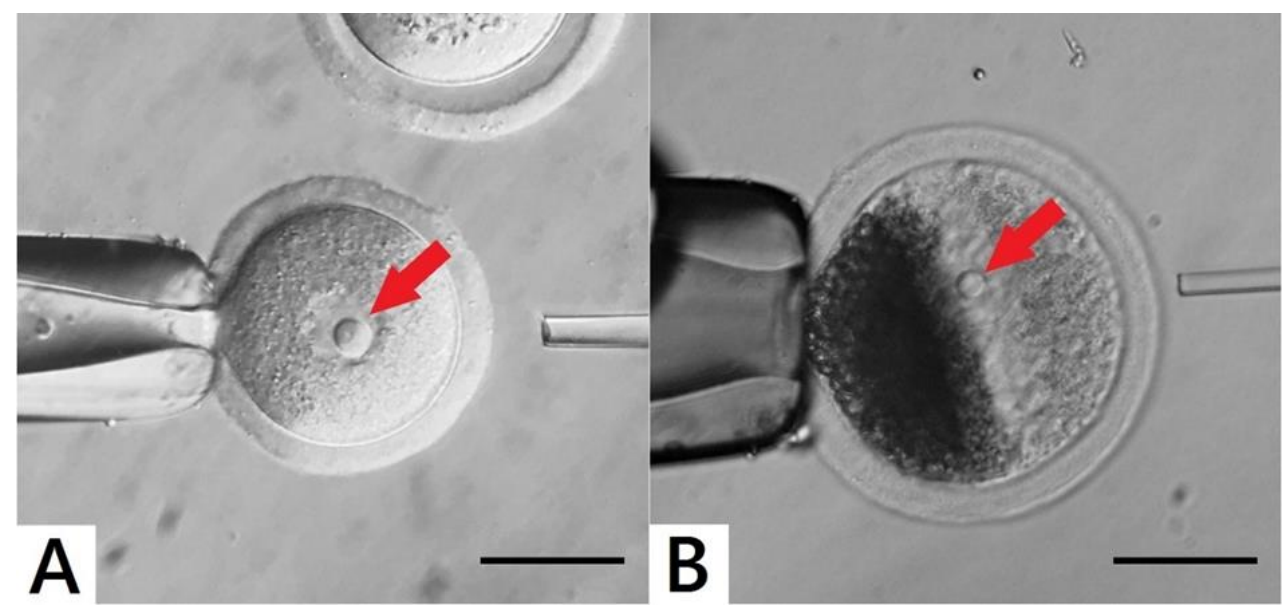


Figure 9.2. Visualisation of NLB in germinal vesicles (GV-red arrow). (A): Mouse oocyte. (B): As porcine oocytes contain many dark lipid particles they must be centrifuged in order to visualize $G V$ with NLBs. Images were produced by the Olympus IX71 inverted microscope. The sample A was originated from porcine large antral follicle and the sample $B$ was originated from mouse large antral follicle of CD - 1 IGS. Scale bar: $50.0 \mu \mathrm{m}$.

Oocytes usually contain a single NLB within the nucleus, however, sometimes more than one NLB can be seen (Fig. 9.3). It is not known, if these oocytes have higher or lower competence to mature or develop after fertilization, but according to our preliminary studies they are not suitable for enucleolation by micromanipulation. It has been commonly accepted that oocyte and zygote NLB serve as repository of material from which differentiated nucleoli in embryos are gradually formed during embryogenesis (Chouinard, L. A. 1975; Chouinard, L. A., 1971). Recent observations have challenged this developmental biology dogma and showed that they are important only during a very short time period after fertilization (Fulka, H., Langerova, A., 2014; Kyogoku, H. et al., 2014).

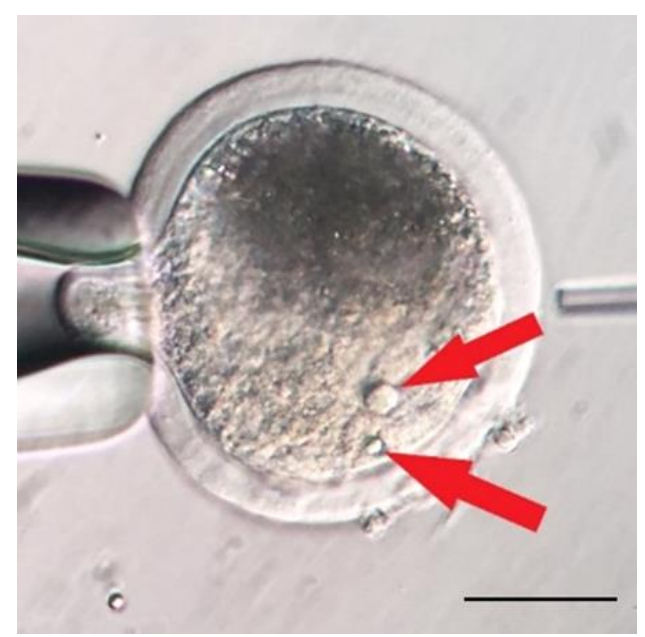

Figure 9.3. Two NLBs in GV stage of porcine oocyte. Image was produced by the Olympus IX71 inverted microscope. The sample was originated from porcine large antral follicle. Scale bar: 50.0 $\mu m$.

During the oocyte growth profound chromatin and nucleolus modifications can be observed. In growing oocytes chromatin is dispersed throughout the germinal vesicle $(\mathrm{GV})$ with no close association with the nucleolus (non-surrounded nucleolus; NSN). On the other hand, in fully grown oocytes more intimate association between the NLB and chromatin can be seen (Christians, E. et al., 1999). The chromatin in fully grown oocytes is in a close contact with the NLB and forms the ring or the horseshoe around it - this is called as the surrounded nucleolus. (SN) (Fig. 9.4) (Bachant, J. B., Elledge, S. J.,1999). Oocytes without close association with heterochromatin can be fertilized or 
parthenogenetic activated, even they form pronuclei but reach only the two-cell stage (Inoue, A. et al., 2008).

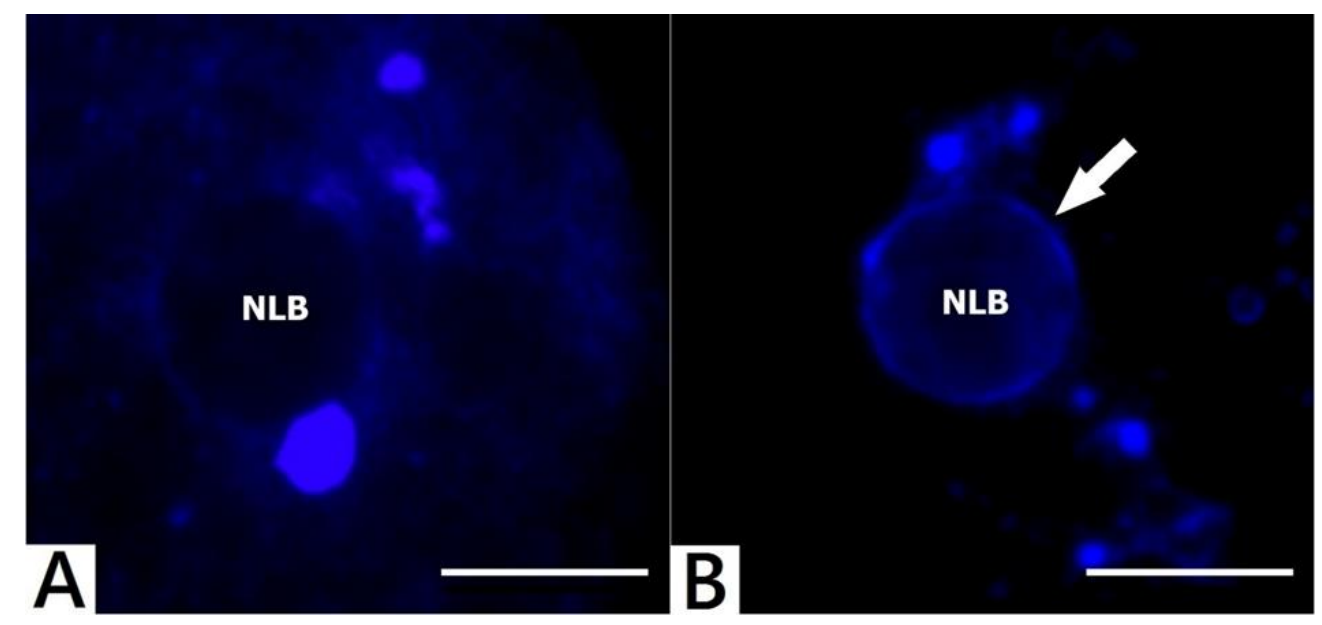

Figure 9.4. Distinct NLB and chromatin association in mouse oocytes. (A): NSN dispersed chromatin organisation. (B): SN chromatin organisation (chromatin - white arrow). Images were produced by the Olympus BX61 confocal fully automated microscope. Fluorescent staining of chromatin was made by Hoechst 33342 (Sigma 14533). All samples were originated from mouse large antral follicles of CD-1 IGS. Scale bar: $10.0 \mu \mathrm{m}$.

It has been shown, that a single mouse NLB isolated from a fully grown oocyte contains approximately $1.6 \pm 0.3 \mathrm{ng}$ of proteins (Fulka, H. et al., 2012). However, only little is known about the proteomic composition of NLBs.

During the oocyte maturation NLB dissolves concomitantly with germinal vesicle breakdown (GVBD) (Snustad, D. P., Simmons, M. J., 2009).

\subsection{Nucleoli in zygotes and early cleavage-stage embryos}

In comparison to immature oocytes at the GV stage, the zygotes typically contain several nucleoli in each pronucleus. During pronuclear development, these nucleoli fuse and form a single prominent structure (Kyogoku, H. et al., 2014). As it has been commonly accepted until recently that this structure is gradually transformed during subsequent embryonic development into typical tripartite nucleoli it was also termed as so called "nucleolus precursor bodies - NPBs".

NPBs in zygotes and early cleavage-stage embryos are strictly of maternal origin, they are not formed de novo and they are essential for chromatin remodelling shortly post oocyte fertilization or parthenogenetic activation (Ogushi, S. et al., 2008). Accordingly, they are not involved in embryonic ribosome production. Important results obtained by NLB and NPB transfer are summarized in the Figure 9.5 (Fulka, H. et al., 2015). 
The NPB in zygotes or in early cleavage-stage embryos differ morphologically from nucleoli in more advanced embryos, stem cells and somatic cells (Fulka, H. et al., 2015). However, in many respects they are very similar to oocyte NLBs because they have dense fibrillar morphology. On the other hand, reticulated nucleoli in somatic cells and advanced embryo developmental stages have typical fibrillo-granular composition (Flechon, J. E., Kopecny, V.,1998; Derenzini, M., et al., 1990; Olson, M. O. J. etal., 2000).

At the two cell stage, mouse embryos become transcriptionally active and begin to synthesize rRNA (Schultz, R. M., 2002; Zatsepina, O. et al., 2003). Throughout this process, NPBs are gradually transformed into reticulated nucleoli (Kyogoku, H.et al., 2014).

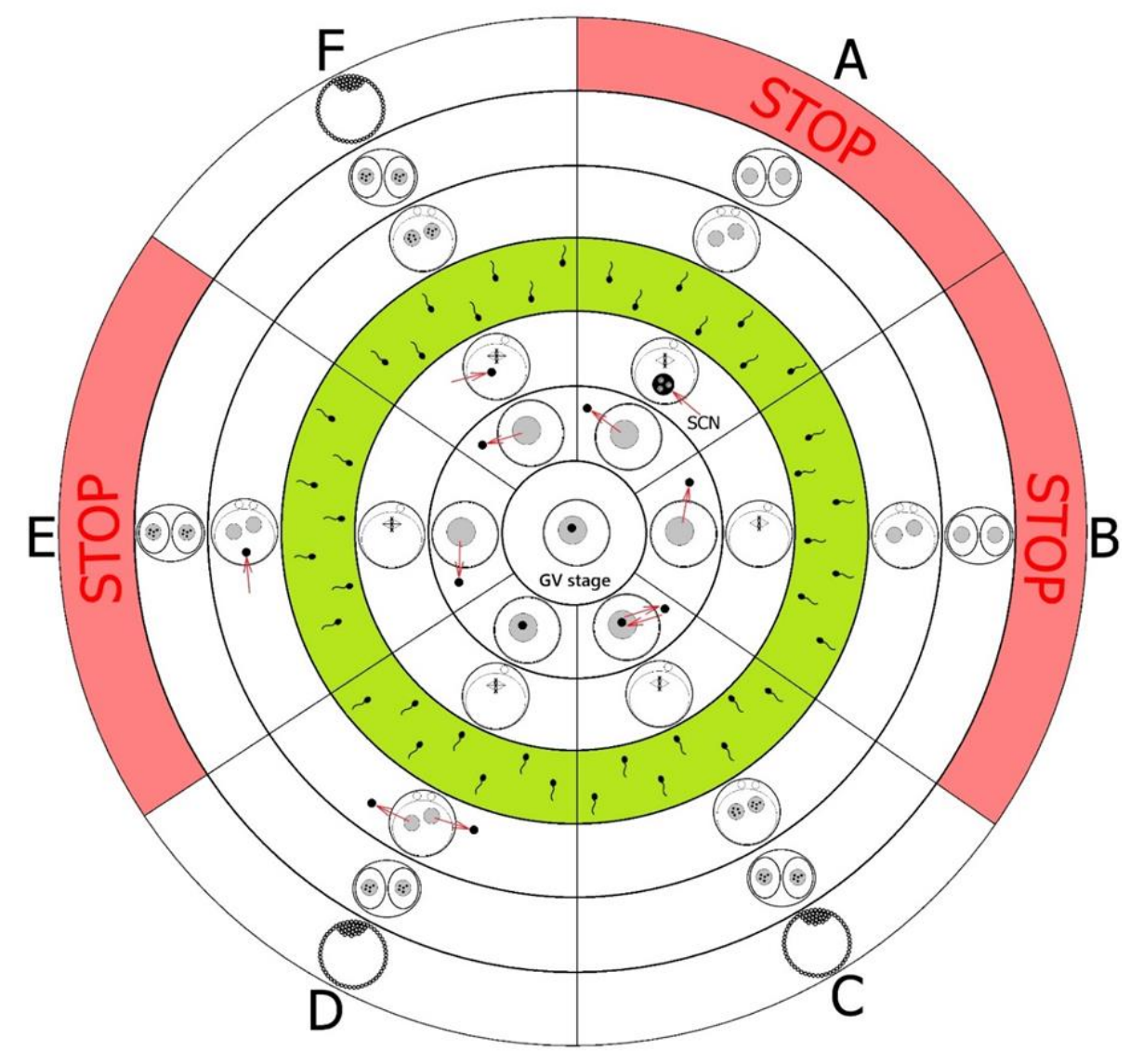

Figure 9.5. Scheme of NLB transfer (nucleolotransfer). SCN-somatic cell nucleus with its nucleolus; Green circle - IVF or parthenogenetic activation; STOP - no development. (A): If NLBs were replaced by somatic cell nucleoli (SCN), the embryo development was stopped and no NPBs in pronuclei were found after parthenogenetic activation or IVF (Ogushi, S.et al., 2008; Wakayama, T. et al., 1999; Wakayama, T., Yanagimachi, R., 2001). (B): Oocytes from which NLBs were removed have matured to metaphase II, however after parthenogenetic activation or IVF no NPBs in pronuclei were found and the embryo development was stopped at the two-cell stage (Ogushi, S.et al., 2008). $(C)$ : If NLBs were re-injected immediately after the enucleolation, these oocytes matured to metaphase II and after parthenogenetic activation or IVF, NPBs in pronuclei were formed and embryos developed to full term (Ogushi, S.et al., 2008). (D): When NPBs were removed from late 
zygotes, no NPBs were detected in pronuclei or nuclei of enucleolated zygotes or in two-cell embryos. However, later (approximately at the four-cell stage) these embryos formed nucleoli de novo and the embryos have developed into the blastocyst stage after parthenogenetic activation or IVF (Kyogoku, $H$ et al., 2008). (E): If NLBs were removed from oocytes and introduced back into more advanced parthenogenetic or IVF embryos (originated from enucleolated oocytes, i.e. more than 8 hrs post activation or IVF), the embryo development was stopped at the two-cell stage (Ogushi, S., Saitou, M., 2010). (F): If NLBs were removed from oocytes and introduced back into less advanced embryos (without NPBs) post parthenogenetic activation or IVF (i.e. less than 7 hrs), NPBs in pronuclei were seen and embryos developed to full term (Ogushi, S.et al., 2008; Ogushi, S., Saitou, M., 2010).

\subsection{Nucleoli in differentiated cells}

Typical differentiated nucleolus in somatic cells is an intranuclear organelle consisting of FC, FCS and GC (Fig. 9.1a) (Hernandez-Verdun, D. et al., 2010). The main function of these nucleoli is the participation on synthesis of ribosomal RNAs (rRNAs) except for 5S RNA. This synthesis is catalysed by RNA polymerase I, primarily located in the nucleolus (Snustad, D. P., Simmons, M. J., 2009). Other functions of these nucleoli include the control of cell cycle progression, genome maintenance, differentiation and regulation of DNA damage responses differentiation etc. (Fulka, H. et al., 2015). rRNA is major structural and functional component of ribosomes. It is created by transcription of rDNA. The cell contains four types of rRNA, which are $120-5000$ base pairs length and present $80 \%$ of all RNA (Koolman, J., Röhm, K. H., 2009).

During the early embryonic development NLB changes its fibrillar composition into a tripartite one. This process is associated with the resumption of rRNA synthesis (Fulka, H. et al., 2015).

\subsection{Nucleolotransfer and its application}

Whilst the somatic cell nucleoli were analysed in detail, very little is known about the composition of NLBs (NPBs). This is a consequence of difficult isolation of this structure from GV oocytes (or zygotes). This has been solved when Fulka, Jr. et al. invented the method of microsurgical removal of NLB from fully grown oocytes called "the enucleolation" (Fulka, J. Jr.et al., 2003).

Shortly after the enucleolation, the volume of isolated NLB rapidly decreases and NLBs dissolve in the medium. However, if NLBs are removed from GVs as so called "nucleoloplasts" (i.e. NLB is enclosed with a small volume of the cytoplasm and cytoplasmic membrane), NLB is protected and does not dissolve. All steps of the procedure are described in detail in the Figure 9.6. It is very important to note, that the isolated NLB is not enclosed with a residual DNA. This was verified by labelling the nucleoloplasts with anti-trimethyl H4/K20 antibody or staining by Hoechst 33342 (Fulka, H. et al., 2012). 


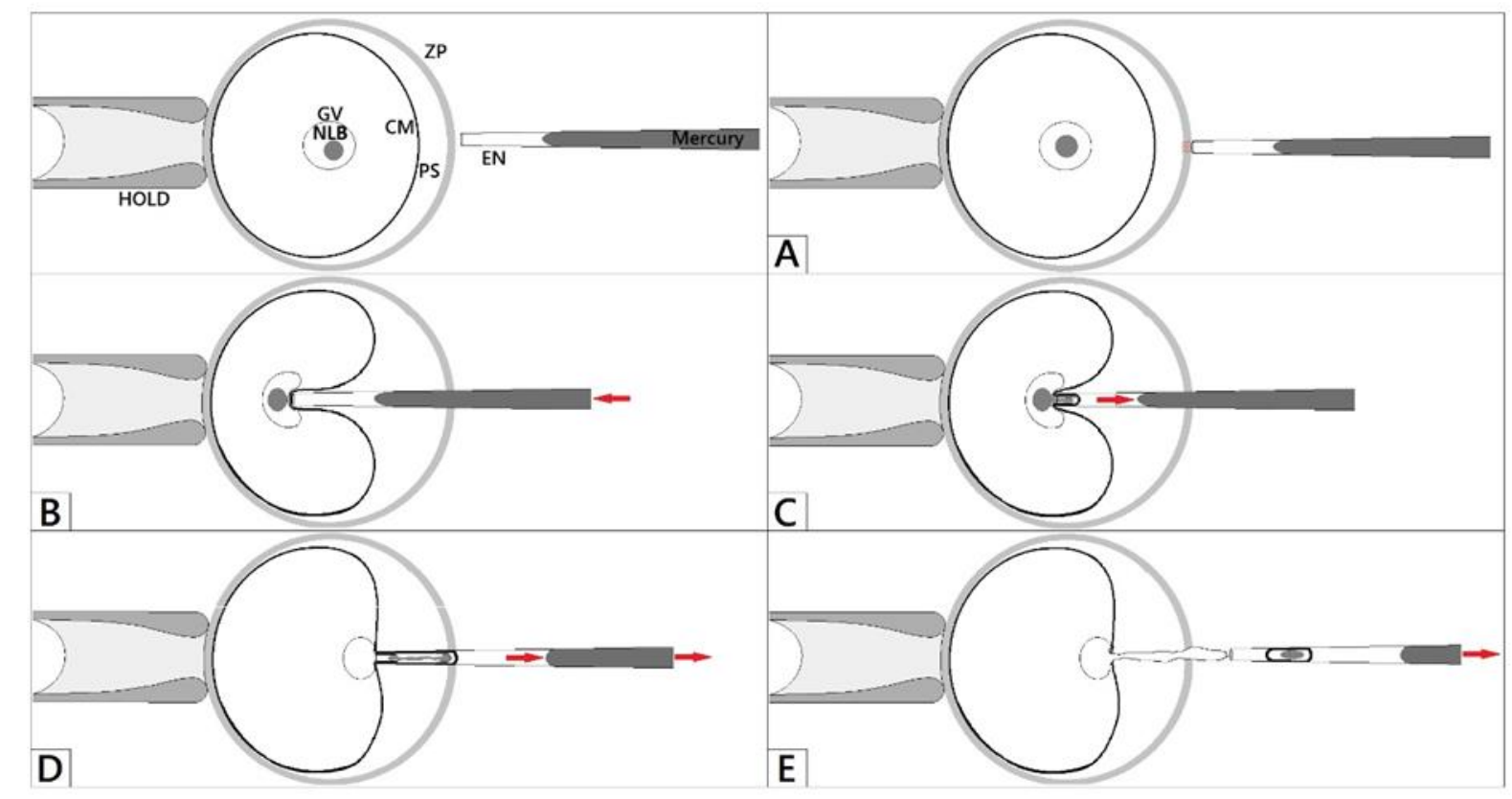

Figure 9.6. Process of enucleolation. HOLD - holding pipette, EN-enucleolation pipette, $Z P$ - zona pellucida, PS - perivitelline space, CM - cytoplasmic membrane, GV-germinal vesicle, NLB. (A): Oocyte is fixed in the stable position by applying the suction in holding pipette. The enucleolation pipette is positioned close to the NLB and penetrates through the zona pellucida. (B): The enucleolation pipette aspirates the $N L B$, but the cytoplasmic membrane is still intact. $(C)$ : The $N L B$ is slowly and gently aspirated into the enucleolation pipette. $(D)$ : The enucleolation pipette is then slowly withdrawn from the oocyte. (E): As soon as the enucleolation pipette is outside, additional suction is applied and the NLB slowly penetrates the germinal vesicle like a sand in a sand-glass.

As mentioned above, the collection of sufficient number of NLBs which can be collected in one experiment is rather complicated, i.e. the enucleolation needs certain skills, NLBs can be very easily lost due to their small diameter or if not collected as nucleoloplasts they dissolve rapidly in the medium. This problem has been solved by producing so called "giant NLBs" (Fig. 9.7) (Fulka, H. et al., 2012). The giant nucleoli are well visible, relatively easily handled and collected and thus ready for further analyses (Fulka, H. et al., 2012). 


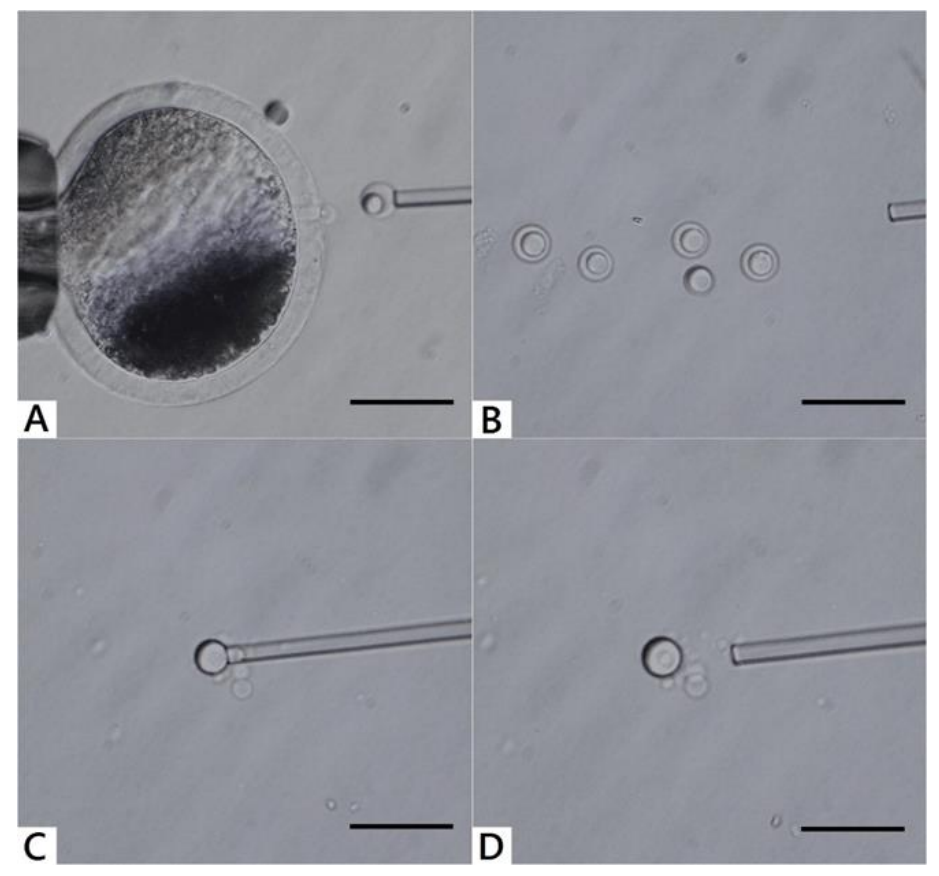

Figure 9.7. Enucleolation and production of giant NLB. (A, B): First step of this process is the microsurgical removal of nucleoli from fully grown oocytes as nucleoloplasts. $(C)$ : Then, nucleoloplasts are aspirated into an enucleolation pipette and the piezo pulses are applied. Consequently, the nucleoloplast envelope (membrane) is broken down and NLB is freed into the manipulation medium. These steps must be then repeated very quickly with remaining nucleoloplasts. (D): Thereafter, the nucleoli are fused when contact between them is achieved and the giant NLB is produced. Image was produced by the Olympus IX71 inverted microscope. Microsurgical processes were made by Narishige micromanipulators with piezo unit (Prime Tech, Tsuchiura, Ibaraki, Japan). The sample was originated from porcine large antral follicle. Scale bar: $50.0 \mu \mathrm{m}$.

Usually, the manipulation medium contains bovine serum albumin (BSA), what may be a problem for further, i.e. proteomic analyses. Therefore, it can be replaced with polyvinyl alcohol (PVA) (Fulka, H. et al., 2012). According to our experiences, for enucleation, the M2 medium (Zenith Biotech ZFM2-050; Czech Republic) supplemented with PVA (Sigma P8136; Czech Republic) is superior to M2 medium (Zenith Biotech ZFM2-050; Czech Republic) supplemented with BSA (Sigma A3311; Czech Republic), as the cytoplasmic membrane appears to be more compact. The enucleolation from zygotes is similar to the method reported for the oocytes (Kyogoku, H. et al., 2014). 
Abbreviations

BSA - bovine serum albumin ()

DFCs - fibrillar components

FCs - fibrillar centers

GC - granular component

$\mathrm{GV}$ - germinal vesicle

GVBD - germinal vesicle breakdown

NLBs - nucleolus-like bodies

NPBs - nucleolus precursor bodies

NSN - non-surrounded nucleolus

$\mathrm{SN}$ - surrounded nucleolus 


\section{Epigenetics}

During development of multicellular organisms, different cells and tissues acquire different programmes of gene expression. It is thought that this is mainly regulated by epigenetic modifications such as DNA methylation, histone tail modifications and non-histone proteins that bind to chromatin Bird (2002), Li (2002). For most cell types in the body, these epigenetic marks become fixed when the cells differentiate or exit the cell cycle. In normal developmental or disease situations, some cells may undergo major epigenetic reprogramming, involving the removal of epigenetic marks in the nucleus, followed by establishment of a different set of marks Rideaut et al. (2001) (Fig. 10.1).

\section{Epigenetic reprogramming cycle}

\section{Gametogenesis Preimplantation Development}

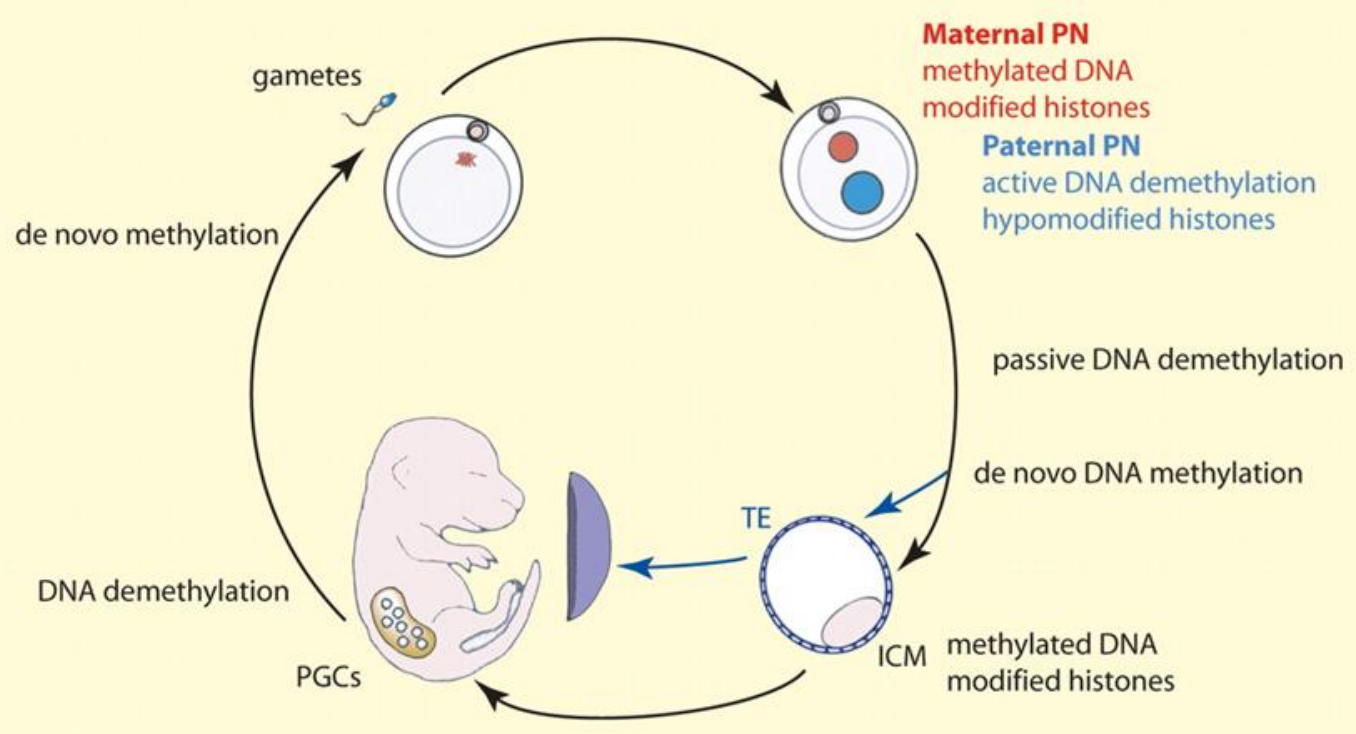

Figure 10.1: Epigenetic reprogramming cycle Hugh et al. (2005)

Epigenetic modifications undergo reprogramming during the life cycle in two phases: during gametogenesis and preimplantation development. Primordial germ cells (PGCs) arise from somatic tissue and develop into mature gametes over an extended period of time. Their genome undergoes DNA demethylation in the embryo between E11.5 and E12.5, including at imprinted genes. Following demethylation, the genomes of the gametes are de novo methylated and acquire imprints; this process 
continues up to E18.5 in males and in maturing oocytes before ovulation in females. Fertilization begins the second round of reprogramming during preimplantation development. The paternal genome is actively demethylated and its histones initially lack some modifications present in the maternal pronucleus (PN). The embryo's genome is passively DNA demethylated during early cell cycles before blastulation. Despite this methylation loss, imprinted genes maintain their methylation through this preimplantation reprogramming. De novo methylation roughly coincides with the differentiation of the first two lineages of the blastocyst stage, and the inner cell mass (ICM) is hypermethylated in comparison to the trophectoderm (TE). These early lineages set-up the DNA methylation status of their somatic and placental derivatives. Histone modifications may also reflect this DNA methylation asymmetry. Particular classes of sequences may not conform to the general genomic pattern of reprogramming shown.

\subsection{DNA methylation}

DNA methylation in animals is associated with transcriptional repression and occurs mainly at the fifth position of cytosine (5mC) in the dinucleotide CpG [12] (Fig. 10.2).

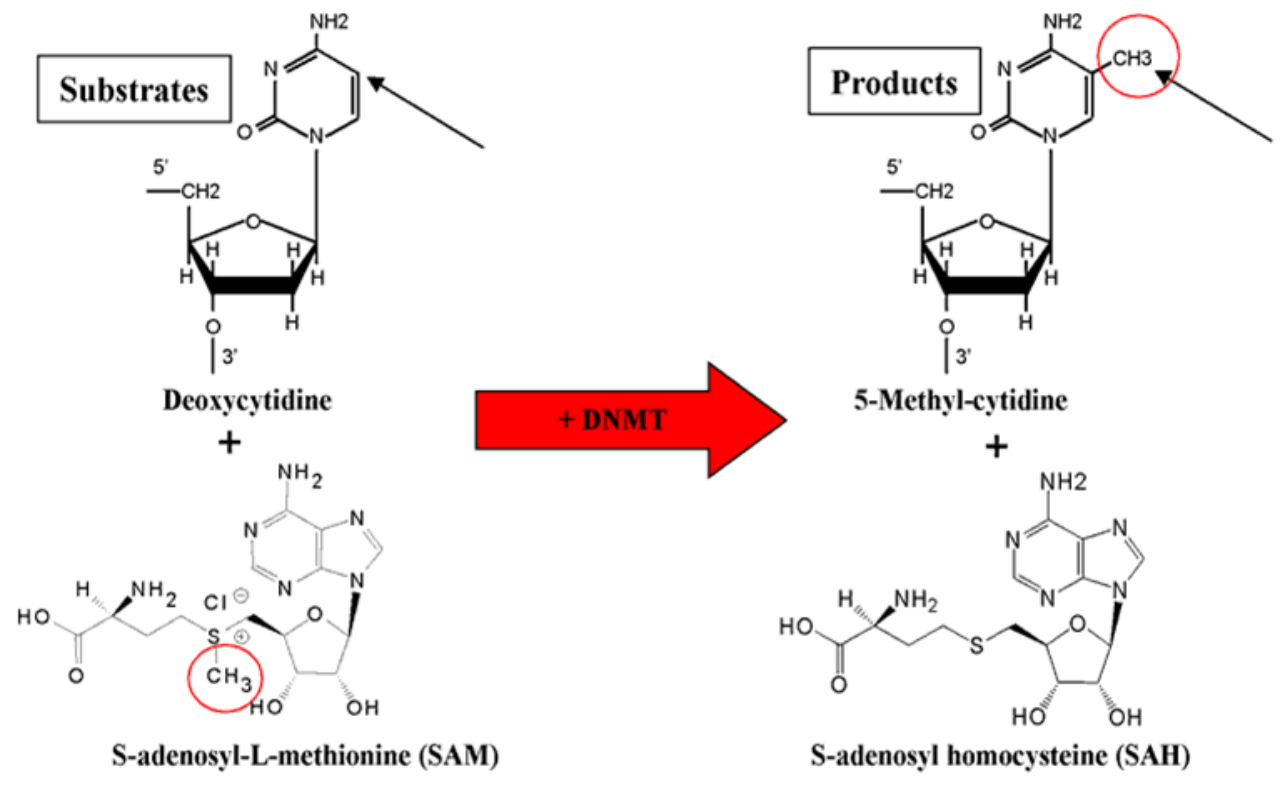

Figure 10.2: DNA methylation reaction catalyzed by DNA methyltransferase (DNMT) (Robertson, 2001).

Over $70 \%$ of CpGs are methylated in vertebrate DNA but distinct patterns can be observed between different somatic and germline tissues Howlett and Reik (1991). These inherited patterns of methylation were hypothesised early on to have functional consequences and early studies indicated 
that there was a correlation between the methylation status of a gene and its expression status Weintraub (1985).

DNA methylation critically depends on the activity of specific enzymes, the DNA methyltransferases (DNMTs) (Fig. 10.3A). Up to five mammalian cytosine DNA methyltransferases (Dnmt) have been identified to date, all of which contain a highly conserved C-terminal catalytic domain and a variable N-terminal extension Bestor (2000). DNA-methyltransferase 1 (DNMT1) (Fig. $10.3 \mathrm{~B}$ ) is a maintenance enzyme that is responsible fo restoring methylation of hemi-methylated $\mathrm{CpG}$ dinucleotides after DNA replication Bestor (1992). An oocyte-specific form, Dnmt1o, is present at high levels in mature oocytes and persists during early zygotic stages. Gene targeting experiments suggest that Dnmt1o has a role in maintaining methylation marks at maternally imprinted genes in mice Howell et al. (2001).

Additional members of the vertebrate cytosine methyltransferase family are DNMT3a and DNMT3b (Fig. 10.3B), which were identified in silico by screening mouse and human databases with sequences corresponding to the catalytic domain Okano et al. (1998). These enzymes catalyze de novo methylation and are thus critical for establishing DNA methylation during development.

DNMT3L co-localizes with DNMT3a and DNMR3b and may co-operate with Dnmt3 family methyltransferases to carry out de novo methylation of maternally imprinted genes during oogenesis and early mouse development.

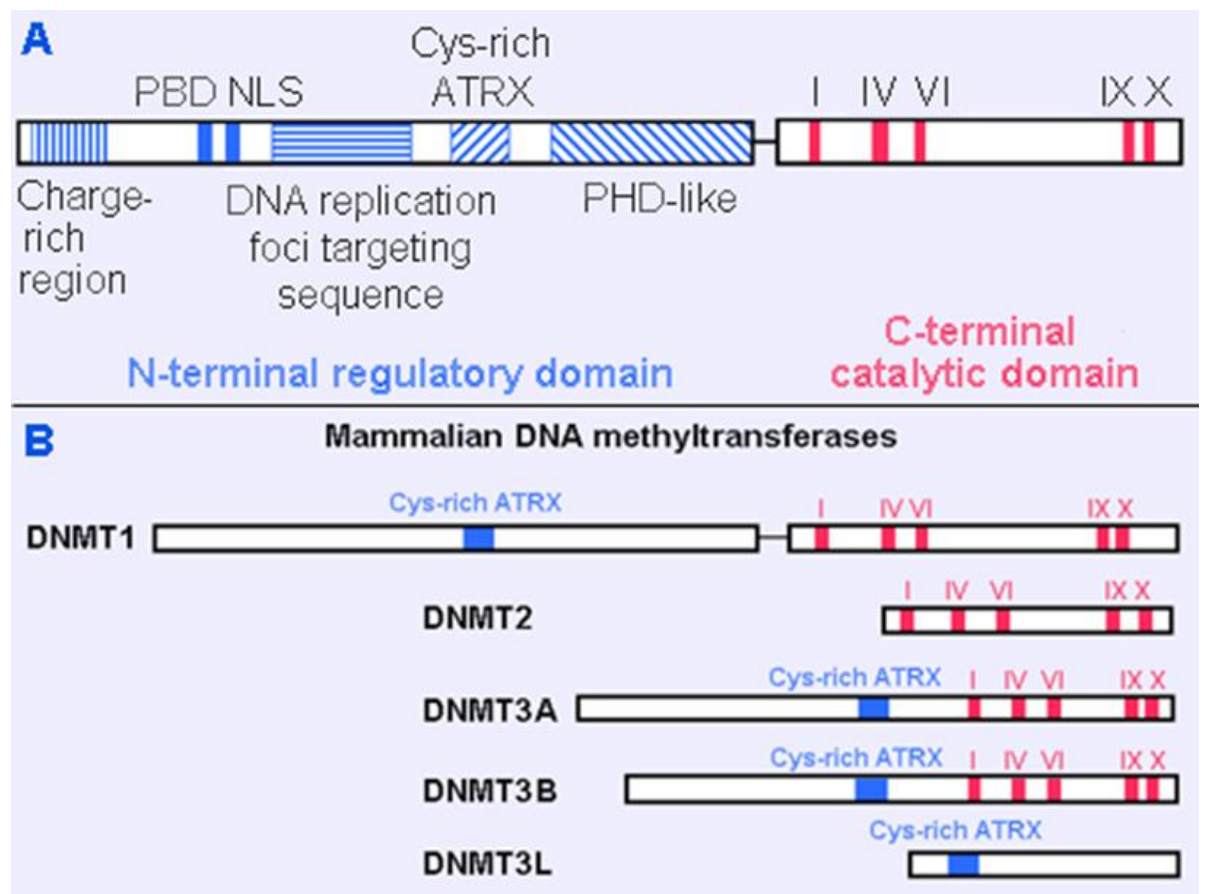

Figure 10.3: A: Structure of mammalian DNMT1 protein. Charge-rich region, the site of interaction with DMAP1; PBD, proliferating cell nuclear antigen-binding domain; NLS, nuclear localization signal; cysteine-rich ATRX, zinc finger DNA-binding motif; PHD-like, polybromo homology domain 
targeting DNMT1 to the replication foci. I, IV, VI, IX and X are conserved motifs of the C-terminal catalytic domain of DNMTs.

B: Homology. (Atlas of Genetics and Cytogenetics in Oncology and Haematology)

DNMT activities are linked with histone deacetylases (HDACs), histone methyltraferases (HMTs) and several ATPases and are part of a complex system regulating chromatin structure and thus gene expression Burgers et al. (2002).

\subsection{Histone modifications}

Histones are the main protein component of chromatin and the core histones $(\mathrm{H} 2 \mathrm{~A}, \mathrm{H} 2 \mathrm{~B}, \mathrm{H} 3$ and H4) form nucleosome. Covalent post-translational modifications of histones play a crucial role in controlling the capacity of the genome store, release and inherit biological information Fischle et al. (2003). Different histone and chromatin related regulatory options are available, including histone acetylation, phosphorylation and metylation (Fig. 10.4).

$\begin{array}{ccccc}\begin{array}{c}\text { Lysine } \\ \text { acetylation }\end{array} & \begin{array}{c}\text { Lysine } \\ \text { methylation }\end{array} & \begin{array}{c}\text { Arginine } \\ \text { methylation }\end{array} & \begin{array}{c}\text { Serine } \\ \text { phosphorylation }\end{array} & \begin{array}{c}\text { Lysine } \\ \text { ubiquitylation }\end{array} \\ \mathrm{K} & \mathrm{K} & \mathrm{R} & \mathrm{S} & \mathrm{K} \\ \text { I } & \mathrm{I} & \mathrm{I} & \mathrm{I} & \mathrm{I} \\ \mathrm{AC} & \mathrm{Me} & \mathrm{Me} & \mathrm{P} & \mathrm{Ub}\end{array}$

Figure 10.4: Types of histone covalent modifications and interacting domains. Types of modifications: acetylation at Lys $(K)$, phosphorylation at Ser $(S)$, methylation at Arg and Lys (R and $K)$ and ubiquitylation at Lys $(K)$. The two classes of domains that interact with specific modified residues are bromodomain $(B r D)$, which interact with acetlyated lysine and chromodomain (ChrD), which interacts with methylated lysine, Berger (2002).

Specific histone methyltransferases (HMTs) catalyze methylation at specific positions of the nucleosome in mammalian cells. Deacetylation of histones is carried out by isoforms of histone deacetylases (HDACs). These enzymes are involved in diverse processes including transcriptional activation, gene silencing, DNA repair and cell-cycle progression and thus play a critical role in cell growth and development. 


\subsection{Epigenetic reprogramming}

Reprogramming can be divided into the pre-zygotic phase, which includes acquisition of genomic imprints and the epigenetic modification of most somatic genes during gametogenesis and post-zygotic reprogramming which includes X-chromosome inactiovation.

At fertilization, the parental genomes are in different stages of the cell cycle with very different epigenetic marks and chromatin organization. The paternal genome has been delivered by the mature sperm, is single copy (1C), and is packaged densely for the most part with protamines rather than histones. In contrast, the maternal genome is arrested at metaphase II with its $2 \mathrm{C}$ genome packaged with histones. Upon fertilization, protamines in sperm chromatin are rapidly replaced with histones, whereas the maternal genome completes meiosis. Closely following histone acquisition of the paternal genome is the initiation of genome wide loss of DNA methylation. This demethylation is completed before DNA replication begins in the paternal pronucleus. The mechanism and the function of paternal genome demethylation are unknown. It is likely that the oocyte cytoplasm contains demethylation factors and that these are specifically targeted to or excluded from certain sequence classes in sperm chromatin. Paternal demethylation may have arisen in order to reprogramme paternal germline imprints by the maternally produced oocyte cytoplasm Reik and Walter (2001).

From the one cell to blastocyst stage there are further changes in global DNA methylation and histone modifications. DNA methylation is reduced progressively with cleavage divisions. This loss of DNA methylation depends on DNA replication and results in unequally methylated sister chromatids Rougier (1998). Many different types of sequences lose methylation at this stage but imprinted genes retain their germline imprints.

\subsection{Methods for detecting DNA methylation}

Methods for DNA methylation are mainly based on the following techniques:

(1) Affinity purification of methylated DNA.

(2) Digestion with methylation-sensitive restriction enzymes.

(3) Bisulfite conversion.

\section{Afinity purification}

In this method, methylated DNA is purified using either a methyl-CpG binding domain (MBD) or, more commonly, an antibody specific for methylated cytosine Weber et al. (2005). Genomic DNA 
fragments containing methylated cytosines are immunoprecipitated and analyzed by hybridization to microarrays or next-generation sequencing. Microarray approach can provide whole-genome coverage, but the resolution is limited by the resolution of the microarrays used. In case of sequencing method the sequence of the affinity-purified DNA fragments is obtained and aligned to a reference genome. This method offers higher resolution compared to microarray but single nucleotide resolution is still not provided by this approach, because it is based on affinity purification of segments of methylated DNA. If a fragment contains several cytosine nucleotides, methylation of any one of them will result in enrichment of that fragment in the affinity purification step.

\section{Methylation-sensitive restriction enzymes}

Methylation-sensitive restriction enzymes cleave specific DNA sequences in a methylationsensitive manner. Some methylation-sensitive restriction enzymes, such as HpaII (which recognizes the sequence 50-. . .CCGG. . .-30), cleave only unmethylated DNA, which others, such as MspI (which recognizes the same 50. . .CCGG. . .-30 sequence), cleave only methylated DNA. These enzymes are the basis of restriction landmark genomic scanning (RLGS), which has been used to assess global DNA methylation Hatada et al. (1991).

In RLGS, genomic DNA is cleaved using a methylation-sensitive restriction enzyme (such as NotI, which does not cleave at recognition sites that are methylated), end-labeled, and run on a 2dimensional gel (Fig. 10.5). Only unmethylated recognition sites are represented in the RLGS map. If a spot is present in the profile for one cell type (e.g., normal cells) and absent in another (e.g., tumor cells), this indicates that a recognition site is unmethylated in the normal cells and methylated in the tumor cells. RLGS reveals large numbers of restriction landmarks in a single experiment, but is limited by the difficulty in correlating individual spots to specific sequences. 


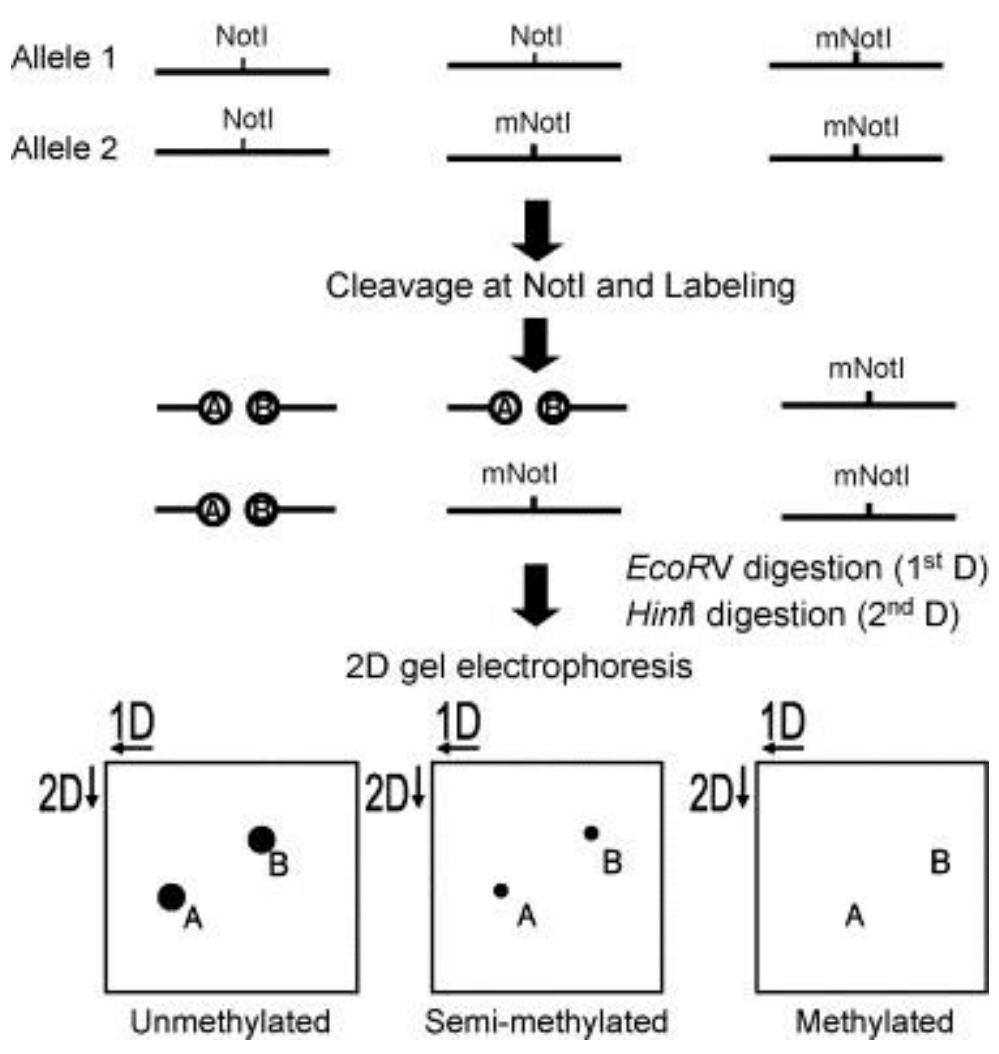

Figure 10.5: Diagram showing the procedures of restriction landmark genomic scanning (RLGS). Methylation detection in the RLGS profile depends on the methylation sensitivity of NotI. NotI, which recognizes CG-rich regions and cannot cleave DNA sequences when 5-cytosine is methylated, acts as the landmark in the profiles. Three types of methylation status are expected, depending on which allele is methylated. mNotI represents the methylated site of the enzymes. Following further endonuclease digestion of EcoRV (1D gel electrophoresis) and HinfI (2D gel electrophoresis), the $R L G S$ profile will result in a change in spot intensity that quantitatively reflects the copy number and methylation status of the NotI fragment. Ho and Tang (2007)

\section{Bisulfite conversion}

Bisulfite conversion is currently one of the most widely used techniques for DNA methylation analysis. First described by Frommer et al. [1992], this technique involves the treatment of DNA with sodium bisulfite, which deaminates unmethylated cytosines (C), converting them into uracils (U). Methylated cytosines are not changed by bisulfite treatment. By comparing the sequences of unconverted and converted DNA, it is possible to identify unmethylated sites (where C's in the unconverted DNA are read as T's (thymine) in the converted DNA) and methylated sites (which are read as C's in both the unconverted and converted DNA) (Fig. 10.6). This technique depends on highly efficient bisulfite conversion (current methods result in unmethylated $\mathrm{C}$ to $\mathrm{U}$ conversion levels of greater than 99\%), and can provide single-nucleotide resolution data. 


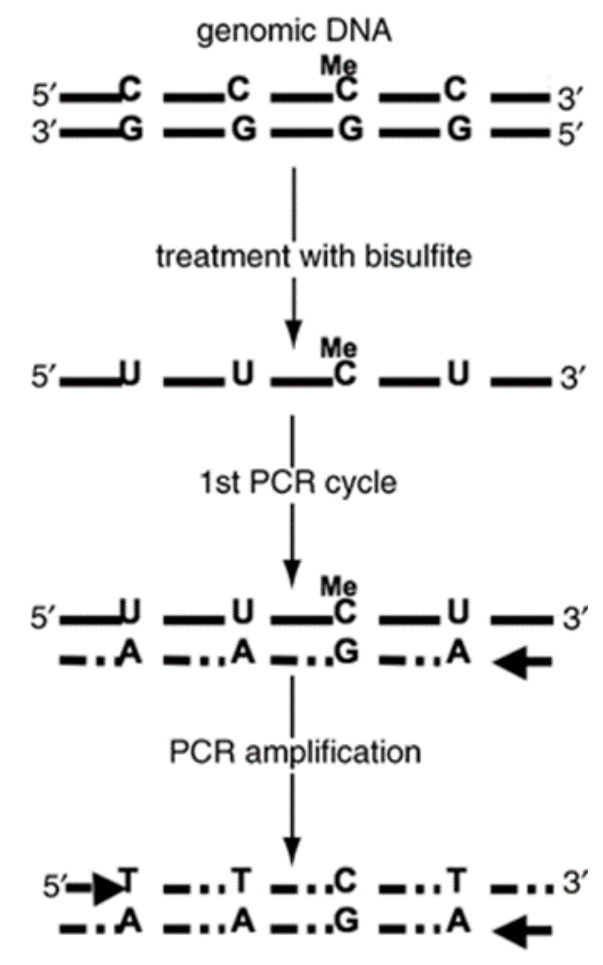

Figure 10.6: Bisulfite conversion

\section{Analysis of bisulfite-converted DNA}

Methylation-specific PCR (MSP).

In methylation-specific PCR (MSP), the bisulfite-converted DNA is subjected to quantitative PCR with primers specific for methylated versus unmethylated DNA [Herman et al., 1996]. This can give an accurate measurement of the

ratio of unmethylated C's to methylated C's. This method can provide single-nucleotide resolution, but is restricted to selected areas of the genome. It can be very useful for interrogating specific sequences of interest.

\section{MethyLight}

MethyLight is a fluorescence-based, quantitativereal-time PCR (qRT-PCR) method developed by Eads et al. (2000). Bisulfite-converted DNA is used as the template for qRT-PCR reactions using TaqMan primer-probes designed against the methylated and unmethylated versions of the target sequence. The high sensitivity of this method enables the detection of a very low abundance of hypermethylated alleles. 


\section{Bisulfite sequencing}

Bisulfite sequencing is the application of any sequencing method to bisulfite-converted DNA. Bisulfite sequencing has long been used to obtain single base-pair resolution DNA methylation data for targeted areas of the genome using conventional sequencing methods, but it was not until the development of next-generation sequencing technology that bisulfite sequencing of large regions of the genome could feasibly be performed.

Abbreviations

DNMTs - DNA methyltransferases

HMTs - methyltransferases

ICM - inner cell mass

MSP - Methylation-specific PCR

PGCs - primordial germ cells

PN - maternal pronucleus

qRT-PCR - quantitativereal-time PCR

RLGS - landmark genomic scanning

TE - trophectoderm 


\section{Summary}

During the past thirty years, experimental studies on basic (superovulation; non-surgical recovery of oocytes and embryos; cryopreservation and transfer of mammalian embryos) and advanced embryo technologies (in vitro embryo production; cloning by somatic cell nuclear transfer) have generated structural and functional information on oocyte development and quality, fertilisation and embryonic development. This information has provided new insight, not only into these technologies but also into the factors contributing to fertility of experimental and farm animals. It is now known that the peripheral and follicular endocrine profiles along with oocyte quality have a profound influence on the subsequent developmental competence of the embryos. It is also well established that manipulation of the oocytes or embryos may negatively affect embryonic and foetal development, leading to the so-called 'large offspring syndrome'. Information from such studies has also alerted scientists to the importance of epigenetics in animal reproduction.

Progress and changes in embryo technology in farm animals are briefly reviewed in terms of how well embryos can be made and used and what the subject has taught us about the maintenance of pregnancy and reproduction in general. Generalizations are made about the need to not accept dogma, how initially complex techniques always become simplified and thereby more applicable, and the need for the support of long-term and basic research. Personal views are offered on how best to prepare and motivate the next generation of scientists in the field, and the need for scientists to engage in the debate of how embryo technologies should be used responsibly in countering global inequalities. 


\section{References}

Abeydeera LR. (2002). In vitro production of embryos in swine. Theriogenology; 57: 257-273.

Ali A, Sirard M. A. (2002). Effect of the absence or presence of various protein supplements on further development of bovine oocytes during in vitro maturation. Biol Reprod 66(4):901-905.

Ali A. A, Bilodeau J. F, Sirard M. A. (2003). Antioxidant requirements for bovine oocytes varies during in vitro maturation, fertilization and development. Theriogenology 59(3-4):939-949.

Amann, R.P. (1986). Detection of alterations in testicular and epididymal function in laboratory animals. Environ. Health Perspect. 70, 149-158.

Anakwe O.O., Sharma S., Hoff H.B., Hardy D.M., Gerton G.L. (1991). Maturation of guinea pig sperm in the epididymis involves the modification of proacrosin oligosaccharide side chains. Mol. Reprod. Dev. 29:294-301.

Ann Van Soom and Marleen Bojerjan (Editors). (2002). Assessment of mammalian embryo quality. Kluwer Academic Publishers (Dordrecht/Boston/London), p. 406.

Aoki, F., Worrad, D.M., Schultz, R.M. (1997). Regulation of transcriptional activity during the first and second cell cycle in the preimplantation mouse embryo. Dev. Biol. 181:296-307.

Arav A (1992). Vitrification of oocytes and embryos. In: Lauria A, Gandolfi F eds, New Trends in Embryo Transfer, Portland Press, Cambridge UK, pp 255-264.

Arav A, Zeron Y, Ocheretny A (2000). A new device and method for vitrification increases the cooling rate and allows successful cryopreservation of bovine oocytes. Theriogenology 53, 248.

Bachant, J. B., Elledge, S. J. (1999). Cell cycle: Mitotic treasures in the nucleolus. Nature. 398, 757758.

Bao, S., Obata, Y., Ono, Y., Futatsumata, N., Niimura, S., Kono, T. (2002). Nuclear competence for maturation and pronuclear formation in mouse oocytes. Human Reproduction 17 (5): 1311-1316.

Bavister BD. (1995). Culture of preimplantation embryos: facts and artifacts. Hum Reprod Update, 1:91-148.

Bedford J, Mock O, Phillips D.(1997). Unusual ampullary sperm crypts, and behavior and role of the cumulus oophorus, in the oviduct of the least shrew, Cryptotis parva. Biol Reprod. 56:12551287.

Beker-van Woudenberg A. R, van Tol H. T, Roelen B. A, Colenbrander B, Bevers M. M. (2004). Estradiol and its membrane-impermeable conjugate (estradiol-bovine serum albumin) during in vitro maturation of bovine oocytes: effects on nuclear and cytoplasmic maturation, cytoskeleton, and embryo quality. Biol Reprod 70(5):1465-1474.

Berisha B, Schams D. (2005). Ovarian function in ruminants. Domest Anim Endocrinol. 29:305-317. 
Bermudez D., Escalier D., Gallo J.M., Viellefond A., Rius F., Perezde Vargas I., Schrevel J. (1994). Proacrosin as a marker of meiotic and postmeiotic germ cell differentiation: quantitative assessment of human spermatogenesis with a monoclonal antibody. J. Reprod. Fertil. 100:567575.

Berthelot F, Martinat-Botté F, Perreau et al. (2001). Birth of piglets after OPS vitrification and transferof compacted morula stage embryos with intact zona pellucida. Reprod. Nutr. Dev. 41, 267-272.

Bestor TH. Activation of mammalian DNA methyltrasferase by cleavage of $\mathrm{Zn}$ binding regulatory domain. EMBO J. 11 1992, 2611-2617

Bestor TH. The DNA methyltransferases of mammals. Hum Mol Genet 2000;9:2395-402.

Betteridge, K. J. (2006). Farm animal embryo technologies: Achievements and perspectives. Theriogenology, 65, 905-913.

Bevers M. M, Izadyar F. (2002). Role of growth hormone and growth hormone receptor in oocyte maturation. Mol Cell Endocrinol 197(1-2):173-178.

Bielanski A, Bergeron H, Lau PCK et al. (2003). Microbial contamination of embryos and semen during long-term banking in liquid nitrogen. Cryobiology 46, 146-152.

Bird, A. (2002) DNA methylation patterns and epigenetic memory. Genes Dev., 16, 6-21.

Bols P. E, Ysebaert M. T, Van Soom A, de Kruif A. (1997). Effects of needle tip bevel and aspiration procedure on the morphology and developmental capacity of bovine compact cumulus oocyte complexes. Theriogenology 47(6):1221-1236.

Booth, P.J., Vajta, G., Høj, A., Holm, P., Jacobsen, H., Greve, T., Callesen, H., (1999). Full-term development of nuclear transfer calves produced from Open Pulled Straw (OPS) vitrified cytoplasts. Theriogenology 51, 99-1006.

Bredbacka K, Bredbacka P. (1996). Glucose controls sex-related growth rate differences of bovine embryos produced in vitro. J Reprod Fertil, 106:169-172.

Bredbacka P, Kankaanpää A, Peippo J. (1995). PCR-sexing of bovine embryos: a simplified protocol. Theriogenology 44:167-76

Briggs R, King TJ (1952). Nuclear transplantation studies on the early gastrula (Rana pipiens). Develop. Biol. 2: 252-270.

Burdon T., Wall R.J., Shamay A., Smith G.H., Hennighausen L. (1991). Over-expression of an endogenous milk protein gene in transgenic mice is associated with impaired mammary alveolar development and a milchlos phenotype. Mech Dev. Dec;36(1-2):67-74.

Burgers, W.A., Fuks, F., and Kouzarides, T. DNA methyltrasferases get connected to chromatin. Trends Genet., 2002, 18: 275-277 
Buy M. (1997). Editorial Assistant, Canadian Council on Animal Care. (From CCAC Resource Supplement, Spring/Summer. Used with permission of CCAC).

Clermont, Y. (1963). The cycle of the seminiferous epithelium in man. Am. J. Anat. 112, 35-51.

Cognie Y. (1999). State of the art in sheep-goat embryo transfer. Theriogenology 51(1):105-116.

Cotcchio G., Sereni E., Serrao L., Mazzone S, Iadarola I., Borini A. (2004). What Criteria for the Definition of Oocyte Quality? Ann N Y Acad Sci. Dec;1034:132-44

Crichton EG, Bedows E, Miller-Lindholm AK et al. (2003). Efficacy of porcine gonadotropins for repeated stimulation of ovarian activity for oocyte retrieval and in vitro embryo production and cryopreservation in Siberian tigers (Panthera tigris altaica). Biology of Reproduction 68, 105113.

Cross N.L. (1998)Role of cholesterol in sperm capacitation. Biol. Reprod.59:7-11.

Dahmus, M.E. (1996). Reversible phosphorylation of the C-terminal domain of RNA polymerase II. J. Biol. Chem. 271:19009-19012.

Dalvit G. C, Cetica P. D, Pintos L. N, Beconi M. T. (2005). Reactive oxygen species in bovine embryo in vitro production. Biocell 29(2):209-212.

Derenzini, M., Thiry, M., Goessens, G. (1990). Ultrastructural cytochemistry of the mammalian cell nucleolus. J. Histochem. Cytochem. 38, 1237-1256.

Dieleman, S.J., Colenbrander, B., Booman, P. (1992). Clinical trends and basic research in animal reproduction. Amsterdam- London- New York- Tokyo : Elsevier 466 p, ISBN 0-444-89734-8.

Dinnyes A, Yang X, Nagai T, Bagis H, Li H, Presicce GA, Gasparrini B, Neglia G, Wilmut I. (2001). Solid surface vitrification (SSV): An efficient method for oocyte and embryo cryopreservation in cattle, pig, and mouse. Cryobiology 43:332.

Diskin MG, Austin EJ, Roche JF. (2002). Exogenous hormonal manipulation of ovarian activity in cattle. Domest Anim Endocrinol. 23:211-228.

Donnay I, Feugang J. M, Bernard S, Marchandise J, Pampfer S, Moens A, Dessy F. (2002). Impact of adding $5.5 \mathrm{mM}$ glucose to SOF medium on the development, metabolism and quality of in vitro produced bovine embryos from the morula to the blastocyst stage. Zygote 10(3):189-199.

Du Y, Pribenszky C, Molnár M, Zhang X., Yang H, Kuwayama M, Pedersen AM, Villemoes K, Bolund L, Vajta G: High hydrostatic pressure (HHP): a new way to improve in vitro developmental competence of porcine matured oocytes after vitrification. Reproduction (in press)

Du Y, Zhang Y, Li J, Kragh PM, Kuwayama M, Ieda S, Zhang X, Schmidt M, Bøgh IB, Purup S, Pedersen AM, Villemoes K, Yang H, Bolund L, Vajta G. (2007). Simplified cryopreservation of porcine cloned blastocysts. Cryobiology. Apr;54(2):181-7. 
Eads CA, Danenberg KD, Kawakami K, Saltz LB, Blake C, Shibata D, Danenberg PV, Laird PW. 2000. MethyLight: A high-throughput assay to measure DNA methylation. Nucleic Acids Res 28:E32.

Ebert K.M. (1998). The use of transgenic animals in biotechnology. Int. J. Dev. Biol. 42, 1003-1008.

Esaki, R., Ueda, H., Kurome, M., Hirakawa, K., Tomii, R., Yoshioka, H., Ushijima, H., Kuwayama, M., Nagashima, H. (2004). Cryopreservation of porcine embryos derived from in vitro-matured oocytes. Biol. Reprod. 71, 432-437.

Esfandiari N, Falcone T, Goldberg JM, Agarwal A, Sharma RK. (2007). Heat-shock proteins modulate the incidence of apoptosis and oxidative stress in preimplantation mouse embryos. Fertil Steril. May;87(5):1214-7.

Eyestone W. H, Jones J. M, First N. L. (1991). Some factors affecting the efficacy of oviduct tissueconditioned medium for the culture of early bovine embryos. J Reprod Fertil 92(1):59-64.

Farin P. W, Piedrahita J. A, Farin C. E. (2006). Errors in development of fetuses and placentas from in vitro-produced bovine embryos. Theriogenology 65(1):178-191.

Ferry L, Mermillod P, Massip A, Dessy F. (1994). Bovine embryos cultured in serum-poor oviductconditioned medium need cooperation to reach the blastocyst stage. Theriogenology 42(3):445453.

First, N.N and Parrish, J.J. (1987). In vitro fertilization of ruminants. J. Reprod. Fert.34 (Suppl.): 151165.

Fischle, W., Wang, Y. and Allis, C.D. Binary switches and modification cassettes in hisotne biology and beyond. Nature 2003, 425: 475-479

Flechon, J. E., Kopecny, V. (1998). The nature of the 'nucleolus precursor body' in early preimplantation embryos: a review of fine-structure cytochemical, immunocytochemical and autoradiographic data related to nucleolar function. Zygote. 6, 183-191.

Flesch, F. M. and Gadella, B.M. (2000). Dynamics of the mammalian sperm plasma membrane in the process of fertilization. Biochimica et Biophysica Acta (BBA)- Reviews of Biomembranes 1469 (3):197-235.

Fraser L.R., Monks N.J. (1990) Cyclic nucleotides and mammalian sperm capacitation. J. Reprod. Fertil. 42:9-21.

French AJ, Hall VJ, Korfiatis NT et al. (2002). Viability of cloned bovine embryos following OPS vitrification. Theriogenology 57, 413.

French AJ, Lewis IM, Ruddock NT et al. (2003). Generation of aS1 casein gene transgenic calves by nuclear transfer. Biology of Reproduction 68, 240 
Fujihira T, Kishida R, Fukui Y (2004). Developmental capacity of vitrified immature porcine oocytes following ICSI: effects of cytochalasin B and cryoprotectants. Cryobiology 49, 286-290.

Fujihira T, Nagai H, Fukui Y (2005). Relationship between equilibration times and the presence of cumulus cells, and effect of Taxol treatment for vitrification of in vitro matured porcine oocytes. Cryobiology (in press)

Fuku E, Kojima T, Shioya Y, Marcus GJ, Downey BR. (1992). In vitro fertilization and development of frozen-thawed bovine oocytes. Cryobiology. Aug;29(4):485-92.

Fukui Y, McGowan L. T, James R. W, Pugh P. A, Tervit H. R. (1991). Factors affecting the in vitro development to blastocysts of bovine oocytes matured and fertilized in vitro. J Reprod Fertil 92(1):125-131.

Fulka, H., Kyogoku, H., Zatsepina, O., Langerova, A, Fulka, J. Jr. (2015). Can Nucleoli Be Markers of Developmental Potential in Human Zygotes? Trends Mol Med. 21(11), 663-672.

Fulka, H., Langerova, A. (2014). The maternal nucleolus plays a key role in centromere satellite maintenance during the oocyte to embryo transition. Development. 141, 1694-1704.

Fulka, H., Martinkova. S., Kyogoku, H., Langerova, A., Fulka, J. Jr. (2012). Production of giant mouse oocyte nucleoli and assessment of their protein content. J. Reprod. Dev. 58, 371-376.

Fulka, J. Jr., Moor, R. M., Loi, P., Fulka, J. (2003). Enucleolation of porcine oocytes. Theriogenology. 59, 1879-1885.

Gaddum-Rosse P. (1985) Mammalian gamete interactions: what can be gained from observations on living eggs? Am J Anat 174:347-356.

Gadella, B.M., Rathi, R., Brouwers, J.F.H.M., Stout, T.A.E., Colenbrander, B. (2001). Capacitation and the acrosome reaction in equine sperm. Animal Reproduction Science 68, 249-265.

Galli C, Duchi R, Crotti G, Turini P, Ponderato N, Colleoni S, Lagutina I, Lazzari G. (2004). Production and quality of bovine oocytes and embryos. Vet Res Commun 28 Suppl 1:121-126.

Galli C., Crotti G., Notari C., Turini P., Duchi R., Lazzari G. (2001). Embryo production by ovum pick up from live donors. Theriogenology, 55, 1341-1357.

Garcia A, Salahaddine M. (1998). Effect of repeated ultrasound-guided transvaginal follicular aspiration on bovine oocyte recovery and subsequent follicular development. Theriogenology, 50, 575-585.

Garcia-Garcia R. M, Ward F, Fair S, O'Meara C M, Wade M, Duffy P, Lonergan P. (2006). Development and quality of sheep embryos cultured in commercial G1.3/G2.3 sequential media. Anim Reprod Sci.

Gardner D. K, Pool T. B, Lane M. (2000). Embryo nutrition and energy metabolism and its relationship to embryo growth, differentiation, and viability. Semin Reprod Med 18(2):205-218. 
Gilchrist RB, Ritter LJ, Armstrong DT. (2004). Oocyte-somatic cell interactions during follicle development in mammals. Anim Reprod Sci. 82-83:431-446.

Glenister PH, Lyon MF. (1986). Long-term storage of eight-cell mouse embryos at - 196 degrees C. J In Vitro Fert Embryo Transf. Feb;3(1):20-7.

Gordon J.W., Ruddle F.H. (1981). Integration and stable germ line transmission of genes injected into mouse pronuclei. Science. Dec 11;214(4526):1244-6.

Gordon JW, Scangos GA, Plotkin DJ, Barbosa JA, Ruddle FH (1980). Genetic transformation of mouse embryos by microinjection of purified DNA. Proc. Natl. Acad. Sci. USA 77: 7380-7384.

Gossler A., Doetschman T., Korn R., Serfling E., Kemler R. (1986) Transgenesis by means of blastocyst-derived embryonic stem cell lines. Proc Natl Acad Sci U S A. Dec;83(23):9065-9.

Greve, T., Callesen, H. (2005). Embryo technology: implications for fertility in cattle. Revue scientifique et technique-Office international des épizooties, 24, 405-412

Griffin TJ, Seth G, Xie H, Bandhakavi S, Hu WS. (2007). Advancing mammalian cell culture engineering using genome-scale technologies. Trends in Biotechnology 25, 401-408,.

Gulyas,B.J. (1979). Cortical granules of mammalian eggs. Int. Rev. Cytol. 63: 357-392.

Haley, S. A., Wessel, G. M. (2004) Regulated Proteolysis by Cortical Granule Serine Protease 1 at Fertilization. Molecular Biology of the Cell 15, 2084-2092.

Hamano S, Koikeda A, Kuwayama M, Nagai T. (1992). Full-term development of in vitro-matured, vitrified and fertilized bovine oocytes. Theriogenology. Dec;38(6):1085-90.

Hamawaki A, Kuwayama M, Hamano S (1999). Minimum volume cooling method for bovine blastocyst vitrification. Theriogenology 51, 165.

Harrison R.A.P.(1996). Capacitation mechanisms, and the role of capacitation as seen in eutherian mammals. Reprod. Fertil. Dev. 1996;8:581-594.

Harvey AJ, Kind KL, Pantaleon M, Armstrong DT, Thompson JG. (2004). Oxygen-regulated gene expression in bovine blastocysts. Biol Reprod, 71:1108-1119.

Hatada I, Hayashizaki Y, Hirotsune S, Komatsubara H, Mukai T. A genomic scanning method for higher organisms using restriction sites as landmarks. Proc Natl Acad Sci USA 1991, 88:95239527.

Hernandez-Verdun, D., Roussel, P., Thiry, M., Sirri, V., Lafontaine, D. L. (2010). The nucleolus: structure/function relationship in RNA metabolism. Wiley Interdiscip Rev RNA. 1(3), 415-431.

Hiraoka K, Hiraoka K, Kinutani M, Kinutani K. (2004). Blastocoele collapse by micropipetting prior to vitrification gives excellent survival and pregnancy outcomes for human day 5 and 6 expanded blastocysts. Hum Reprod. Dec;19(12):2884-8.. 
Hochi S, Terao T, Kamei M, Kato M, Hirabayashi M, Hirao M. (2004). Successful vitrification of pronuclear-stage rabbit zygotes by minimum volume cooling procedure. Theriogenology. Jan $15 ; 61(2-3): 267-75$.

Holdcraft, R.W., Braun, R.E. (2004). Hormonal regulation of spermatogenesis. J. Androl. 27:335342.

Hoodbhoy ,T. and Dean, J. (2004). Insights into the molecular basis of sperm-egg recognition in mammals Reprod. 127: 417-422.

Hou YP, Dai YP, Zhu SE, Zhu HB, Wu TY, Gong GC, Wang HP, Wang LL, Liu Y, Li R, Wan R, Li N. (2005). Bovine oocytes vitrified by the open pulled straw method and used for somatic cell cloning supported development to term. Theriogenology. Oct 1;64(6):1381-91.

Houdebine L.M.: Transgenic animal bioreactors. Transgenic Res., 9, 305-320, 2000.

Houdebine L.M.: Transgenic animals. Generation and use. Published in The Netherlands by Harwood Academic publishers. ISBN 90-5702-068-8.

Howell CY, et al. Genomic imprinting disrupted by a maternal effect mutation in the Dnmt1 gene. Cell 2001;104:829-38.

Howlett SK, Reik W. Methylation levels of maternal an paternal genomes during preimplantation development. Development 1991, 113: 119-27.

Hredzak R, Ostro A, Zdilova V (2005). Clinical experience with a modified method of human embryo vitrification Ceska Gynekologica 70, 99-103 (in Slovakian).

Hugh D. Morgan, Fa'tima Santos, Kelly Green, Wendy Dean and Wolf Reik, Epigenetic reprogramming in mammals, Human Molecular Genetics, 2005, Vol. 14, Review Issue 1 47-58

Hunter MG. (2000). Oocyte maturation and ovum quality in pigs. Rev Reprod.5:122-130.

Hunter RH. (1996). Ovarian control of very low sperm/egg ratios at the commencement of mammalian fertilization to avoid polyspermy. Mol. Reprod. Dev. Jul;44(3):417-22.

Hunter, T. (1987). A thousand and one protein kinases. Cell, 50:823-829.

Hyttel, P., Greve, T., Callesen, H. (1988a). Ultrastructure of in vivo fertilization in superovulated cattle. J. Reprod. Fertil. 82:1-13.

Chen C (1986). Pregnancy after human oocyte cryopreservation. Lancet 1(8486): 884-886.

Chen SU, Lien YR, Cheng YY et al. (2001). Vitrification of mouse oocytes using closed pulled straws (CPS) achieves a high survival and preserves good patterns of meiotic spindles, compared with conventional straws, open pulled straws (OPS) and grids. Human Reproduction 11, 2350-2356.

Chouinard, L. A. (1971). A light- and electron-microscope study of the nucleolus during growth of the oocyte in the prepubertal mouse. J. Cell Sci. 9, 637-663. 
Chouinard, L. A. (1975). A light- and electron-microscope study of the oocyte nucleus during development of the antral follicle in the prepubertal mouse. J. Cell Sci. 17, 589-615.

Chrenek P., Makarevich A. (2005). Production of rabbit chimeric embryos by aggregation of zonafree nuclear transfer blastomeres. Zygote 13, 39-44.

Chrenek P., Makarevich A., Vasicek D., Laurincik J., Bulla J., Gajarska T., Rafay J. (1998) Effects of superovulation, culture and microinjection on development of rabbit embryos in vitro. Theriogenology 50, 659-666.

Chrenek P., Vasicek D., Makarevich A., Jurcik R., Suvegova K., Bauer M., Parkanyi V., Rafay J., Batorova A., Paleyanda R.K. (2005). Increased transgene integration efficiency upon microinjection of DNA into both pronuclei of rabbit embryos. Transgenic Res., 14, 417-428.

Chrenek P., Vašíček D., Makarevich A., Jurčík R., Suvegová K., Bauer M., Rafay J., Bulla J., Hetenyi L., Erickson J., Paleyanda R. (2003) Integration rate of exogenous DNA into rabbit genome using single and double pronucleus microinjection. Transgenic Research conference, California, August, poster 101.

Christians, E., Boiani, M., Garagna, S., Dessy, C., Redi, C. A., Renard, J. P., Zuccotti, M. (1999). Gene expression and chromatin organization during mouse oocyte growth. Dev Biol. 207(1), 7685.

Inoue, A. Nakajima, R., Nagata, M., Aoki, F. (2008). Contribution of the oocyte nucleus and cytoplasm to the determination of meiotic and developmental competence in mice. Hum. Reprod. 23, 1377-1384.

Isachenko V, Alabart JL, Vajta G et al. (2000). Double cryopreservation of rat embryos at different developmental stages with identical vitrification protocol: the not properly understood phenomenon. In: Abstracts of the Winter Meeting of Society for the Study of Fertility. Utrecht, Holland. Journal of Reproduction and Fertility 26 (Abstract Series), 10.

Iwayama H, Hochi S, Kato M et al. (2004). Effects of cryodevice type and donor's sexual maturity on vitrification of minke whale (Balaenopter bonaerensis) oocytes at germinal vesicle stage. Zygote 12, 333-338.

Izadyar F, Van Tol H. T, Colenbrander B, Bevers M. M. (1997). Stimulatory effect of growth hormone on in vitro maturation of bovine oocytes is exerted through cumulus cells and not mediated by IGF-I. Mol Reprod Dev 47(2):175-180.

Jaenish R.. (1976). Germ line integration and Mendelian transmission of the exogenous Moloney leukemia virus. Proc. Natl. Acad. Sci. USA, 73, 1260-1264.

Johnson, L., Wilker, C.E., Cerelli, J.S., (1994). Spermatogenesis in the bull. Tech. Conf. Artif. Insem. Reprod.15, 9-27. 
Juraj Pivko. (1995). Morfogenéza oocytov a raných embryí niektorých živočíchov. VÚŽV v Nitre vo vydavatel'stve SAP- Slovak Academic Press, spol. s r.o., Bratislava, p.113.

Kagawa N, Kuwayama M, Nakata K, Vajta G, Silber S, Manabe N, Kato O. (2007). Production of the first offspring from oocytes derived from fresh and cryopreserved pre-antral follicles of adult mice. Reprod Biomed Online. Jun;14(6):693-9.

Kaneko, K.J., DePamphilis, M.L. (1998). Regulation of gene expression at the beginning of mammalian development and the TEAD family of transcription factors. Dev. Genetics 22:43-55.

Kanka, J. (2003). Gene expression and chromatin structure in the pre-implantation embryo. Theriogenology 59:3-19.

Kasai M, Zhu SE, Pedro PB, Nakamura K, Sakurai T, Edashige K. (1996). Fracture damage of embryos and its prevention during vitrification and warming. Cryobiology. Aug;33(4):459-64

Kidson A.: In vitro embryo development in the pig.http://www.vetscite.org/publish/articles/000057/index.html

Killian G. J. (2004). Evidence for the role of oviduct secretions in sperm function, fertilization and embryo development. Anim Reprod Sci 82-83:141-153.

Kobayashi M, Lee E. S, Fukui Y. (2006). Cysteamine or beta-mercaptoethanol added to a defined maturation medium improves blastocyst formation of porcine oocytes after intracytoplasmic sperm injection. Theriogenology 65(6):1191-1199.

Kobayashi S, Takei M, Kano M et al. (1998). Piglets produced by transfer of vitrified porcine embryos after stepwise dilution of cryoprotectants. Cryobiology 36, 20-31.

Kong IK, Lee SI, Cho SG et al. (2000). Comparison of open pulled straw (OPS) vs glass micropipette (GMP) vitrification in mouse blastocysts. Theriogenology 53, 1817-1826.

Koolman, J., Röhm, K. H. (2009). Taschenatlas Biochemie des Menschen. Georg Thieme Verlag Stuttgart, New York, 66.

Kremling H., Keime S., Wilhelm K., Adham I.M., Hameister H., Engel W. (1991) Mouse proacrosin gene: nucleotide sequence, diploid expression, and chromosomal localization. Genomics. $11: 828-834$.

Kubelka M, Motlik J, Fulka JJ, Prochazka R, Rimkevikova Z, Fulka J. (1988).Time sequence of germinal vesicle breakdown in pig oocytes after cycloheximide and p-aminobenzamidine block. Gam. Res.; 19: 423-431.

Kuleshova L, Gianaroli L, Magli C, Trounson A (1999a). Birth following vitrification of a small number of human oocytes. Human Reproduction 14, 3077-3079.

Kuleshova LL, Lopata A 2002 Vitrification can be more favorable than slow cooling. Fertility and Sterility 78, 449-454. 
Kupriyanov S., Zeh K., Baribault H.: Double pronuclei injection of DNA into zygotes increase yields of transgenic mouse lines. Transgenic Res., 7, 1998, 223-226.

Kuwayama M, Kato O (2000). All-round vitrification method for human oocytes and embryos. Journal of Assisted Reproduction and Genetics 17, 477.

Kuwayama M, Vajta G, Ieda S, Kato O (2005b). Vitrification of human embryos using the CryoTip ${ }^{\mathrm{TM}}$ method. Reproductive BioMedicine Online 11, 608-614.

Kuwayama M, Vajta G, Kato O, Leibo SP.. (2005a). Highly efficient vitrification method for cryopreservation of human oocytes. Reproductive BioMedicine Online 11, 300-308. .

Kuwayama M:Highly efficient vitrification for cryopreservation of human oocytes and embryos: the Cryotop method. Theriogenology. 2007 Jan 1;67(1):73-80.

Kyogoku, H. Fulka, J. Jr., Wakayama, T., Miyano, T. (2014). De novo formation of nucleoli in developing mouse embryos originating from enucleolated zygotes. Development. 141, 22552259.

L.S.A. Camargo, J.H.M.Viana, W.F. Sál A.M. Ferreira, A.A. Ramos, V.R. Vale Filho (2006). Factors influencing in vitro embryo production. Anim. Reprod., v.3, n.1, p.19-28.

Lake, James A. and Maria C. Riveral (1999). "Horizontal gene transfer among genomes: The complexity hypothesis". PNAS (Proceedings of the National Academy of Science) 96:7: pp. 3801-3806. Retrieved on 2007-03-18.

Landa V, Tepla O (1990). Cryopreservation of mouse 8-cell embryos in microdrops. Folia Biologica (Praha) 36: 153-158.

Lane M. (2001). Mechanisms for managing cellular and homeostatic stress in vitro. Theriogenology, 55:225-236.

Langlais J., Kan F.W., Granger L., Raymond L., Bleau G., Roberts K.D. (1998). Identification of sterol acceptors that stimulate cholesterol efflux from human spermatozoa during in vitro capacitation. Gamete Res. 20:185-201.

Langlais J., Roberts K.D. (1985). A molecular membrane model of sperm capacitation and acrosome reaction of mammalian spermatozoa. Gamete Res. 12:183-224.

Latham, K.E., Solter, D., Schultz, R.M. (1992). Acquisition of a transcriptionally permissive state during the 1-cell stage of mouse embryogenesis. Dev. Biol. 149:457-462.

Laurinčík, J., Kopečný, V., Hyttel, P. (1996). A detailed analysis of pronucleus development in bovine zygotes in vivo: Ultrastructure and cell cycle chronology. Mol. Reprod. Dev. 43:62-

Lefèvre, B. (2008). The nucleolus of the maternal gamete is essential for life. Bioessays. 30, 613616. 
Leibo SP. (1984). A one-step method for direct nonsurgical transfer of frozen-thawed bovine embryos. Theriogenology. May;21(5):767-90.

Li, E. (2002) Chromatin modification and epigenetic reprogramming in mammalian development. Nat. Rev. Genet., 3, 662-673

Li, R., Lai, L., Wax, D., Hao, Y., Murphy, C.N., Rieke, A., Samuel, M., Linville, M.L., Korte, S.W., Evans, R.W., Turk, J.R., Kang, J.X., Witt, W.T., Dai, Y., Prather, R.S. (2006). Cloned transgenic swine via in vitro production and cryopreservation. Biol. Reprod. 75, 226-230.

Liebermann J, Tucker MJ, Graham JR et al. (2002a). Blastocyst development after vitrification of multipronuclear zygotes using the Flexipet denuding pipette. Reproductive BioMedicine Online $4,146-150$.

Lin L, Du Y, Kragh PM, Li J, Bolund L, Yang H, Zhang X, Kuwayama M, Vajta G: Induced blastocoel collapse improves survival rates of porcine blastocysts after vitrification (2008). Reproduction, Fertility and Development (in press)

Lin Y., Kan F.W. (1996). Regionalization and redistribution of membrane phospholipids and cholesterol in mouse spermatozoa during in vitro capacitation. Biol. Reprod. 55:1133-1146.

Lonergan P, Carolan C, Van Langendonckt A, Donnay I, Khatir H, Mermillod P. (1996). Role of epidermal growth factor in bovine oocyte maturation and preimplantation embryo development in vitro. Biol Reprod 54(6):1420-1429.

Lonergan P. (2007). State-of-the-art embryo technologies in cattle. Soc Reprod Fertil Suppl.64:315325.

Lopez-Bejar M, Lopez-Gatius F (2002). Nonequilibrium cryopreservation of rabbit embryos using a modified (sealed) open pulled straw procedure. Theriogenology 58, 1541-1552.

Maclellan LJ, Carnevale EM, Coutinho da Silva MA et al. (2002). Pregnancies from vitrified equine oocytes collected from super-stimulated and non-stimulated mares. Theriogenology 58, 911-919.

Macmillan KL, Segwagwe BV, Pino CS. (2003). Associations between the manipulation of patterns of follicular development and fertility in cattle. Anim Reprod Sci. 78:327-344.

Makarevich A.V., Chrenek P., Zilka N., Pivko J., Bulla J. (2005). Preimplantation development and viability of in vitro cultured rabbit embryos derived from in vivo fertilized gene-microinjected eggs.:apoptosis and ultrastructure analyses. Zygote 13, 125-137.

Manual of the International Embryo Transfer Society (IETS) (1998). (third edition, D.A. Stringfellow and S.M. Seidel, Eds.), IETS, p.170.

Martino A, Songsasen N, Leibo SP (1996b). Development into blastocysts of bovine oocytes cryopreserved by ultra-rapid cooling. Biology of Reproduction 54, 1059-1069. 
Martino A. Pollard JA, Leibo SP (1996a). Effect of chilling bovine oocytes on their developmental competence. Molecular Reproduction and Development 45, 503-512

Massip A, Van Der Zwalmen P, Scheffen B, Ectors F: Pregnancies following transfer of cattle embryos preserved by vitrification. Cryo-Letters 7: 270-273

Matsumoto T., Komori K., Shoji T., Kuma S., Kume M., Yamaoka T., Mori E., Furuyama T., Yonemitsu Y., Sugimachi K. (2001). Successful and optimized in vivo gene transfer to rabbit carotid artery mediated by electronic pulse. Gene Ther. Aug;8(15):1174-9.

Mayes M. (2002). The meiotic arrest of bovine oocytes. Faculte des Sciences de L’Agriculture et de L`Alimentation Universite Laval Quebec.

Mazur P. (1970). Cryobiology: the freezing of biological systems. Science. May 22;168(934):93949.

McCracken, S., Fong, N., Yankulov, K., Ballantyne, S., Pan, G., Greenblatt, J., Patterson, S.D., Wickens, M., Bentley, D.L. (1997). The C-terminal domain of RNA polymerase II couples mRNA processing to transcription. Nature 385:357-361.

McLachlan RI, O’Donnell L, Meachem SJ, Stanton PG, De Kretser DM, Pratis K, Robertson DM. (2002). Hormonal regulation of spermatogenesis in primates and man: insights for development of the male hormonal contraceptive. J. Androl. 23:149-162.

Meirelles, F.V., Caetano, A.R., Watanabe, Y.F., Ripamonte, P., Carrambula, S.F., Merighe, G.K., Garcia, S.M. (2004). Genome activation and developmental block in bovine embryos. Anim. Reprod. Sci. 82-83:13-20.

Memili, E., First, N.L. (1999). Control of gene expression at the onset of bovine embryonic development. Biol. Reprod. 61:1198-1207.

Men H, Agca Y, Critser E et al. (2005). Beneficial effects of serum supplementation during in vitro production of porcine embryos on their ability to survive cryopreservation by the open pulled straw vitrification. Theriogenology 64, 1340-1349.

Mermillod P., Massip A., Dessy F. (1992). In vitro production of cattle embryos: review and Belgian results., int.J.Dev.Biol., 36, 185-195.

Moreno RD, Ramalho-Santos J, Chan EK, Wessel GM, Schatten G. (2000) The Golgi apparatus segregates from the lysosomal/acrosomal vesicle during rhesus spermiogenesis: structural alterations. Dev Biol 219:334-349.

Morgan, H.D., Santos, F., Green, K., Dean, W., Reik, W. (2005). Epigenetic reprogramming in mammals. Hum. Mol. Gen. 14:47-58

Mori, T., Guo M. W., Sato, E.,Baba, T., Takasaki, S. Mori, E. (2000) Molecular and immunological approaches to mammalian fertilization Journal of Reproductive Immunology 47, 139-158. 
Mortillo S., Wassarman P.M (1991). Differential binding of gold-labeled zona pellucida glycoproteins $\mathrm{mZP} 2$ and $\mathrm{mZP} 3$ to mouse sperm membrane compartments. Development 113:141-149.

Mukaida T, Oka C, Goto T, Takahashi K. (2006). Artificial shrinkage of blastocoeles using either a micro-needle or a laser pulse prior to the cooling steps of vitrification improves survival rate and pregnancy outcome of vitrified human blastocysts. Hum Reprod. Dec;21(12):3246-52.

Myers M. W, Broussard J. R, Menezo Y, Prough S. G, Blackwell J, Godke R. A, Thibodeaux J. K. (1994). Established cell lines and their conditioned media support bovine embryo development during in-vitro culture. Hum Reprod 9(10):1927-1931.

Nagashima H, Kashiwazaki N, Ashman RJ et al. (1994). Removal of cytoplasmic lipid enhances the tolerance of porcine embryos to chilling. Biology of Reproduction 51, 618-22.

Nagy A, Rossant J, Nagy R, Abramow-Newerly W, Roder JC (1993). Derivation of completely cell culture-derived mice from early-passage embryonic stem cells. Proc. Natl. Acad. Sci. USA 90:8424-8.

Nakagata N. (1989). High survival rate of unfertilized mouse oocytes after vitrification. J Reprod Fertil. Nov;87(2):479-83.

Ng SC, Bongso A, Chang SI, Sathananthan H, Ratnam S (1989). Transfer of human sperm into the perivitelline space of human oocytes after zona-drilling or zona-puncture. Fertil. Steril. 52: 7378.

Ng SC, Bongso TA, Ratnam SS. (1990). Micro-insemination: genetic aspects. Arch Androl. 25: 261270.

O'Rand M.G. (1982). Modification of the sperm membrane during capacitation.Ann N Y Acad. Sci. 383:392-404.

Ogushi, S., Palmieri, C., Fulka, H., Saitou, M., Miyano, T., Fulka, J. Jr. (2008). The maternal nucleolus is essential for early embryonic development in mammals. Science. 319, 613-616.

Ogushi, S., Saitou, M. (2010). The nucleolus in the mouse oocyte is required for the early step of both female and male pronucleus organization. J. Reprod. Dev. 56, 495-501.

Okano M, Xie S, Li E. Cloning and characterization of a family of novel mammalian DNA (cytosine5) methyltransferases. Nat Genet 1998;19:219-20.

Olson S. E, Seidel G. E, Jr. (2000). Reduced oxygen tension and EDTA improve bovine zygote development in a chemically defined medium. J Anim Sci 78(1):152-157.

Olson, M. O. J., Dundr, M., Szebeni A. (2000). The nucleolus: an old factory with unexpected capabilities. Trends Cell Biol. 10, 189-196. 
P. Mermillod, Y. Locatelli, R. Dalbiès-Tran, S. Uzbekova, G. Baril, F. Guignot, C. Perreau, N. Poulin, J. L. Touzé, S. Pennetier, B. Schmaltz, and Y. Cognié (2006). In vitro production of ruminant embryos: Results, limits and perspectives., Symposium COA/INRA Scientific Cooperation in Agriculture, Tainan (Taiwan, R.O.C.), November 7-10

Palermo G, Joris H, Evroey P, Van Steirteghem AC (1992). Pregnancies after intracytoplasmic injection of single spermatozoon int an oocyte. Lancet 340: 17-18.

Palmiter R.D., Brinster R.L. (1986). Germ-line transformation of mice. Annu Rev Genet.;20:465-99. Review.

Palmiter R.D., Brinster R.L., Hammer R.E., Trumbauer M.E., Rosenfeld M.G., Birnberg N.C., Evans R.M. (1982). Dramatic growth of mice that develop from eggs microinjected with metallothionein-growth hormone fusion genes. Nature. Dec 16;300(5893):611-5.

Parrish, J.J., Susko-Parrish, J.L, Leibfried-Rutledge, M.L, Critser, E.S.,Eystone, W.H. and First, N.L. (1986). Bovine in vitro fertilization with frozen thawed semen. Theriogenology 25: 591-600.

Petters RM, Wells KD. (1993). Culture of pig embryos. J Reprod Fertil Suppl; 48: 61-73.

Peura TT, Hartwich KM, Hamilton HM et al. (2003). No differences in sheep somatic cell nuclear transfer outcomes using serum starved or actively growing donor granulosa cells. Reproduction, Fertility and Development 15, 157-165.

Pieterse M. C, Kappen K. A, Kruip T. A, Taverne M. A. (1988). Aspiration of bovine oocytes during transvaginal ultrasound scanning of the ovaries. Theriogenology 30(4):751-762.

Piltty K, Lindeberg H, Aalto J et al. (2004). Live cubs born after transfer of OPS vitrified-warmed embryos in the farmed European polecat (Mustela putorius). Theriogenology 61, 811-820.

Pinkert C.A. (2002). Transgenic animal technology. A laboratory handbook. Academic press (An imprint of Elsevier Science), USA, Second edition, , p. 601, ISBN 0-12-557166-6.

Pinyopummintr,T. and Bavister, B.D.(1991). In vitro matured/in vitro fertilized bovine oocytes can develop into morulae/blastocysts. In chemically defined protein-free culture media. Bioi Reprod 45: 735-742.

Polge C. (1952) Fertilizing capacity of bull spermatozoa after freezing at 79 degrees C.Nature. Apr 12;169(4302):626-7.

Popova E., Krivokharchenko A., Ganten D., Bader M. (2004). Efficiency of transgenic rat production is independent of transgene-construct and overnight embryo culture. Theriogenology $61,, 1441$ 53.

Pribenszky C, Molnár M, Cseh S, Solti L (2005a). Improving post-thaw survival of cryopreserved mouse blastocysts by hydrostatic pressure challenge. Animal Reproduction Science 87: 143-150. 
Pribenszky C, Molnár M, Ulrich P, Keiko L (2005b). Improving the post-thaw survival of cryopreserved IVP bovine blastocysts by hydrostatic pressure challenge. Reproduction in Domestic Animals 40:1.

Rall WF (1987). Factors affecting the survival of mouse embryos cryopreserved by vitrification. Cryobiology 24 387-402.

Rall WF, Fahy GM (1985). Ice-free cryopreservation of mouse embryos by vitrification. Nature 313, 573-575.

Ramalho-Santos J., Schatten G.,Moreno R.D. (2002). Control of Membrane Fusion During Spermiogenesis and the Acrosome Reaction. Biol. Reprod. 67, 1043-1051.

Rankin T.L., Tong Z-B, Castle P.E., Lee E., Gore-Langton R., Nelson, L.M., Dean J. Human ZP3 restores fertility in Zp3 null mice without affecting order-specific sperm binding (1998) Development 125:2415-2424.

Reik, W. and Walter, J. Evolution of imprinting mechanisms: the battle of the sexes begins in the zygote. Nat. Genet 2001, 27: 255-256.

Reik, W., Dean, W., Walter, J. (2001). Epigenetic reprogramming in mammalian development. Science 293:1089-1093.

Rideout, W.M., III, Eggan, K. and Jaenisch, R. (2001) Nuclear cloning and epigenetic reprogramming of the genome. Science, 293, 1093-1098.

Rieger D. and Betteridge K.J. (1993). Embryo transfer and related techniques in domestic animals, and their implications for human medicine. Hum Reprod. Jan;8(1):147-67. Review. Erratum in: Hum Reprod 1993 Apr;8(4):652.

Rizos D, Gutierrez-Adan A, Perez-Garnelo S, De La Fuente J, Boland M. P, Lonergan P. (2003). Bovine embryo culture in the presence or absence of serum: implications for blastocyst development, cryotolerance, and messenger RNA expression. Biol Reprod 68(1):236-243.

Rizos D, Ward F, Duffy P, Boland M. P, Lonergan P. (2002). Consequences of bovine oocyte maturation, fertilization or early embryo development in vitro versus in vivo: implications for blastocyst yield and blastocyst quality. Mol Reprod Dev 61(2):234-248.

Robertson, K.D. DNA methylation, methyltransferases, and cancer. Oncogene (2001) 20, 3139 - 3155

Rodman, T. C., Bachvarova, R. (1976). RNA synthesis in preovulatory mouse oocytes. J. Cell Biol. $70,251-257$.

Rodriguez-Martinez, (2007). Role of the oviduct in sperm capacitation. Theriogenology 68, 138-145.

Rodriguez-Martinez, H., Saravia, F., Wallgren, M., Tienthai, P., Johannisson, A., Vázquez, J.M. 2005 Boar spermatozoa in the oviduct. Theriogenology 63, 514-35. 
Rougier, N., Bourc'his, D., Gomes, D.M., Niveleau, A., Plachot, M., Paldi, A. and Viegas-Pequignot, E. Chromosome methylation patterns during mammalian preimplantation development. Genes Dev. 1998, 12: 2108-2113

Santos, F., Dean, W. (2004). Epigenetic reprogramming during early development in mammals. Reproduction 127:643-651.

Serrano H., Garcia-Suarez, D. (2001) Molecular aspects of mammalian fertilization Asian J Androl Dec; 3: 243-249.

Shapiro, B.M., Somers, C., and Weidman, P.J. (1989). Extracellular remodeling during fertilization. In: Cell Biology of Fertilization (ed. H. Schatten and G. Schatten), San Diego: Academic Press, $251-276$.

Shelley L Berger, Histone modifications in transcriptional regulation. Current Opinion in Genetics \& Development 2002, 12:142-148

Shuk-mei Ho, Wan-yee Tang. Techniques used in studies of epigenome dysregulation due to aberrant DNA methylation: An emphasis on fetal-based adult diseases. Reproductive Toxicology,Volume 23, Issue 3, 2007, Pages 267-282

Schier, A. (2007). The maternal-zygotic transition: Death and birth of RNAs. Science 316:406-407.

Schultz, R. M. (2002). The molecular foundations of the maternal to zygotic transition in the preimplantation embryo. Hum. Reprod. Update. 8, 323-331.

Smith T.T., McKinnon-Thompson C.A., Wolf D.E. (1998). Changes in lipid diffusibility in the hamster sperm head plasma membrane during capacitation in vivo and in vitro. Mol. Reprod. Dev. 50:86-92.

Snustad, D. P., Simmons, M. J. (2009). Principles of genetics. John Wiley \& Sons, UK, 18.

Soler JP, Mucci N, Kaiser GG, Aller J, Hunter JW, Dixon TE, Alberio RH. (2007). Multiple ovulation and embryo transfer with fresh, frozen and vitrified red deer (Cervus elaphus) embryos in Argentina. Anim Reprod Sci. Mar 30; Epub

Spemann H (1938). Embryonic development és induction. Yale University Press, New Haven, Connecticut, p. 211.

Stitzel, M.L., Seydoux, G. (2007). Regulation of the oocyte-to zygote transition. Science 316:407408.

Sutton-McDowall M. L, Gilchrist R. B, Thompson J. G. (2004). Cumulus expansion and glucose utilisation by bovine cumulus-oocyte complexes during in vitro maturation: the influence of glucosamine and follicle-stimulating hormone. Reproduction 128(3):313-319.

Swierstra, E.E., Gebauer, M.R., Pickett, B.W. (1974). Reproductive physiology of the stallion: I. Spermatogenesis and testis composition. J. Reprod. Fertil. 40, 113-123. 
Talbot P, Shur, B. D. Myles D.G. (2003). Cell Adhesion and Fertilization: Steps in Oocyte Transport, Sperm-Zona Pellucida Interactions, and Sperm-Egg Fusion. Biol.Reprod. 68, 1-9.

Tardif, S., Dube, Ch., Bailey, J.L. (2003). Porcine Sperm Capacitation and Tyrosine Kinase Activity Are Dependent on Bicarbonate and Calcium but Protein Tyrosine Phosphorylation Is Only Associated with Calcium1. Biol. Reprod. 68:207-213.

Tarkowski AK (1961). Mouse chimæras developed from fused eggs. Nat. 4779: 857-860.

Tecirlioglu RT, French AJ, Lewis IM et al. (2004). Birth of a cloned calf derived from a vitrified cloned embryo. Reproduction, Fertility and Development 15, 361-366.

Thompson J. G, Gardner D. K, Pugh P. A, McMillan W. H, Tervit H. R. (1995). Lamb birth weight is affected by culture system utilized during in vitro pre-elongation development of ovine embryos. Biol Reprod 53(6):1385-1391.

Thompson JG. 2000. In vitro culture and embryo metabolism of cattle and sheep embryos - a decade of achievement. Anim Reprod Sci, 60/61:263-275.

Thompson, E.M., Legouy, E., Renard, J.-P. (1998). Mouse embryos do not wait for the MBT: Chromatin and RNA polymerase remodeling in genome activation at the onset of development. Dev. Genetics 22:31-42.

Tominaga K, Hamada Y (2001). Gel-loading tips as container for vitrification of in vitro-produced bovine embryos. Journal of Reproduction and Development 47, 259-265.

Toth, S., Huneau, D., Banrezes, B. and Ozil, J.P. (2006) Egg activation is the result of calcium signal summation in the mouse. Reproduction 131: 27-34.

Trounson A, Mohr L (1983). Human pregnancy following cryopreservation, thawing, and transfer of an eight-cell embryo. Nature 305, 707-709.

Uguz C, Vredenburgh W.L., Parrish J.J. (1994). Heparin-induced capacitation but not intracellular alkalinization of bovine sperm is inhibited by Rpadenosine-3',5'-cyclic monophosphothioate. Biol. Reprod. 51:1031-1039.

Vajta G, Holm P, Kuwayama M, Booth PJ, Jacobsen H, Greve T, Callesen H.(1998a). Open pulled straw vitrification: a new way to reduce cryoinjuries of bovine ova and embryos. Molecular Reproduction and Development 51, 53-58.

Vajta G, Lewis IM, Hyttel P, Thouas GA, Trounson AO (2001). Somatic cell cloning without micromanipulators. Cloning 3: 89-95.

Vajta G, Lewis IM, Kuwayama M, Greve T, Callesen H (1998b). Sterile application of the Open Pulled Straw (OPS) vitrification method. Cryo-Letters 19, 389-392. 
Vajta G, Lewis IM, Trounson AO, Purup S, Maddox-Hyttel P, Schmidt M, Pedersen HG, Greve T, Callesen H (2003). Handmade somatic cell cloning in cattle: Analysis of factors contributing to high efficiency in vitro. Biol. Reprod. 68: 571-578.

Vajta G, Nagy ZP. (2006).Are programmable freezers still needed in the embryo laboratory? Review on vitrification. Reprod Biomed OnlineJun;12(6):779-96.

Van den Abbeel E, Camus M, Verheyen G, Van Waesberghe L, Devroey P, Van Steirteghem A. (2005). Slow controlled-rate freezing of sequentially cultured human blastocysts: an evaluation of two freezing strategies. Hum Reprod. Oct;20(10):2939-45.

Van den Hurk R, Zhao J. (2005). Formation of mammalian oocytes and their growth, differentiation and maturation within ovarian follicles. Theriogenology. 63:1717-1751.

Vanderzwalmen P, Bertin G, Debauche Ch et al. (2002). Births after vitrificaton at morula and blastocyst stages: effect of artificial reduction of the blastocoelic cavity before vitrification. Human Reproduction 17, 744-751

Vanderzwalmen P, Bertin G, Debauche V et al. (2000). In vitro survival of metaphase II oocytes (MII) and blastocysts after vitrification in an hemi-straw (HS) system. Fertility and Sterility 74, S215-216.

Vieria AD, Mezzalira A, Barieri DP et al. Calves born after open pulled straw vitrification of immature bovine oocytes. Cryobiology 45, 91-94.

Visconti, P.E., Galantinohomer, H., Moore, G.D., Bailey, J.L., Ning, X., Fornes, M., Kopf, G.S. (1998). The Molecular Basis of sperm Capacitation. Journal of Andrology 19, (2): 242-248.

Voss A.K., Sandmoller A., Suske G., Strojek R. M., Beato M., Hahn, J. (1990). A comparison of mouse and rabbit embryos for the production of transgenic animals by pronuclear microinjection. Theriogenology, 34, , 813-824.

Wakayama T, Perry ACF, Zuccoti M, Johnson KR, Yamagimachi R (1998). Full term development of mice from enucleated oocytes injected with cumulus cell nuclei. Nat. 394:369-374.

Wakayama, T., Rodriguez, I., Perry, A. C., Yanagimachi, R., Mombaerts, P. (1999). Mice cloned from embryonic stem cells. Proc. Natl. Acad. Sci. U.S.A. 96, 14984-14989.

Wakayama, T., Yanagimachi, R. (2001). Mouse cloning with nucleus donor cells of different age and type. Mol. Reprod. Dev. 58, 376-383.

Wall R.J. (1996). Transgenic livestock: progress and prospects for the future. Theriogenology 45, 5768.

Wall R.J., Seidel G.E. Jr. (1992). Transgenic farm animals - A critical analysis.Theriogenology, 38, 337-57. 
Weber M, Davies JJ, Wittig D, Oakeley EJ, Haase M, Lam WL, Schubeler D. Chromosome-wide and promoter-specific analyses identify sites of differential DNA methylation in normal and transformed human cells. Nat Genet 2005, 37:853-862

Weintraub H. Assembly and propagation of repressed and depressed chromosomal states. Cell 1985, 42: 705-11.

Wessel, G.M., Brooks, J.M., Green, E., Haley, S., Voronina, E., Wong, J., Zaydfudim, V., and Conner, S. (2001). The biology of cortical granules, vol 209. In: International Review of Cytology (ed. K.W. Jeon), San Diego: Academic Press, 117-206.

Whittingham DG, Leibo SP, Mazur P (1972). Survival of mouse embryos frozen to $-196^{\circ} \mathrm{C}$ and $269^{\circ} \mathrm{C}$. Science $178,411-414$.

Whittingham DG. (1977). Fertilization in vitro and development to term of unfertilized mouse oocytes previously stored at --196 degrees C.J Reprod Fertil. Jan;49(1):89-94.

Willadsen SM (1986). Nuclear transplantation in sheep embryos. Nat. 320: 63-5.

Willadsen SM, Polge C, Rowson LE et al. (1976). Deep freezing of sheep embryos. Journal of Reproduction and Fertility 46, 151-154.

Wilmut I, Rowson LEA (1973). Experiments on the low temperature preservation of cow embryos. Veterinary Record 92, 686-690.

Wilmut I, Schnieke AE, McWhir J, Kind AJ, Campbell KH (1997). Viable offspring derived from fetal és adult mammalian cells. Nature 385: 810-3.

Wilson JA, Richardson CD. (2003). Induction of RNA interference using short interfering RNA expression vectors in cell culture and animal systems. Curr Opininions in Mol Therapy 5,389396.

Wolpert, L., Beddington, R., Jessell, ,T., Lawrence, P., Meyerowitz, E., Smith, J. (2002). Principles of development. Oxford University press, New York.

Wong, J., and Wessel, G.M. (2004). Major components in a sea urchin block to polyspermy are structurally and functionally conserved. Evol. Dev. 6, 134-153.

Xu, K.P. and Greve, T. (1988). Detailed analysis of early events during in- vitro fertilization of bovine follicular oocytes. J. reprod. Fertil. 82:127-134.

Yanagimachi, R. (1988a). Mammalian fertilization. In: Knobil, E., et al. (Eds.), The Physiology of Reproduction. Raven, New York, pp. 135-185.

Yanagimachi, R. (1994). Mammalian fertilization. In: Knobil, E., Neill, J.D. (Eds.), The Physiology of Reproduction. Raven Press, New York, pp. 189-317.

Yang X, Kubota C, Suzuki H, Taneja M, Bols PE, Presicce GA. (1998). Control of oocyte maturation in cows--biological factors. Theriogenology; 49: 471-82. 
Yuan YQ, Van Soom A, Coopman FO, Mintiens K, Boerjan ML, Van Zeveren A, De Kruif A, Peelman LJ. (2003). Influence of oxygen tension on apoptosis and hatching in bovine embryos cultured in vitro. Theriogenology, 59:1585-1596.

Zatsepina, O., Baly, C., Chebrout, M., Debey, P. (2003). The step-wise assembly of a functional nucleolus in preimplantation mouse embryos involves the Cajal (coiled) body. Dev. Biol. 253, 66-83.

Zeng Y, Oberdorf J.A.. Florman H.M.(1996). pH regulation in mouse sperm. Identification of Na, $\mathrm{Cl}$ - and $\mathrm{HCO}$-dependent and arylaminobenzoatedependent regulatory mechanisms and characterization of their role in sperm capacitation. Dev. Biol. 173:510-520.

Internet:

„http://users.rcn.com“

,Www.bioprotein.com“

anonymous (2007) http://web2.uwindsor.ca/courses/biology/weis/55-101/lec16a.ppt

http://www.advancedfertility.com/embryoquality.htm 
Title: Embryotechnology II

Authors: Jozef Laurinčík, Martin Morovič, Michal Benc, František Strejček, Matej Murín, Alexander V. Sirotkin, Peter Chrenek, Alexander V. Makarevich, Martin R. Luck, Gábor Vajta, Lazo Pendovski, Ol'ga Ostrup

If the figures are without citation they are author's originals.

Publisher: Constantine the Philosopher University in Nitra, Faculty of Natural Sciences Prírodovedec n. 681

Cover design: František Strejček

Year of publication: 2018

Pages: 200

Print numbers: 50

(C) Faculty of Natural Sciences, Constantine the Philosopher University in Nitra 2018

DOI: http://doi.org/10.17846/2018-embryotechnology

ISBN 978-80-558-1337-0

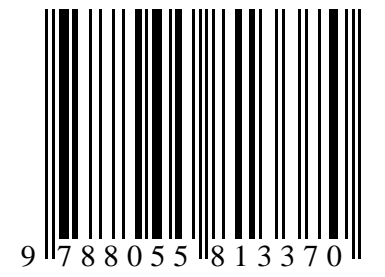

EAN 9788055813370 